\title{
Canadian clinical practice guidelines for the management of anxiety, posttraumatic stress and obsessive-compulsive disorders
}

\author{
Martin A Katzman ${ }^{1 *}$, Pierre Bleau ${ }^{2}$, Pierre Blier ${ }^{3}$, Pratap Chokka ${ }^{4}$, Kevin Kjernisted ${ }^{5}$, Michael Van Ameringen ${ }^{6}$, \\ the Canadian Anxiety Guidelines Initiative Group on behalf of the Anxiety Disorders Association of Canada/ \\ Association Canadienne des troubles anxieux and McGill University
}

\begin{abstract}
Background: Anxiety and related disorders are among the most common mental disorders, with lifetime prevalence reportedly as high as 31\%. Unfortunately, anxiety disorders are under-diagnosed and under-treated.

Methods: These guidelines were developed by Canadian experts in anxiety and related disorders through a consensus process. Data on the epidemiology, diagnosis, and treatment (psychological and pharmacological) were obtained through MEDLINE, PsycINFO, and manual searches (1980-2012). Treatment strategies were rated on strength of evidence, and a clinical recommendation for each intervention was made, based on global impression of efficacy, effectiveness, and side effects, using a modified version of the periodic health examination guidelines.

Results: These guidelines are presented in 10 sections, including an introduction, principles of diagnosis and management, six sections (Sections 3 through 8) on the specific anxiety-related disorders (panic disorder, agoraphobia, specific phobia, social anxiety disorder, generalized anxiety disorder, obsessive-compulsive disorder, and posttraumatic stress disorder), and two additional sections on special populations (children/adolescents, pregnant/lactating women, and the elderly) and clinical issues in patients with comorbid conditions.

Conclusions: Anxiety and related disorders are very common in clinical practice, and frequently comorbid with other psychiatric and medical conditions. Optimal management requires a good understanding of the efficacy and side effect profiles of pharmacological and psychological treatments.
\end{abstract}

\section{Introduction}

Anxiety and related disorders are among the most common of mental disorders. Lifetime prevalence of anxiety disorders is reportedly as high as $31 \%$; higher than the lifetime prevalence of mood disorders and substance use disorders (SUDs) [1-5]. Unfortunately, anxiety disorders are under-diagnosed [6] and under-treated [5,7,8].

These guidelines were developed to assist clinicians, including primary care physicians and psychiatrists, as well as psychologists, social workers, occupational therapists, and nurses with the diagnosis and treatment of anxiety and related disorders by providing practical,

\footnotetext{
* Correspondence: mkatzman@startclinic.ca

'Department of Psychiatry, University of Toronto, Toronto, ON, M5S 1A1,

Canada

Full list of author information is available at the end of the article
}

evidence-based recommendations. This guideline document is not focused on any individual type of clinician but rather on assessing the data and making recommendations. Subsequent "user friendly" tools and other initiatives are planned.

The guidelines include panic disorder, agoraphobia, specific phobia, social anxiety disorder (SAD), generalized anxiety disorder (GAD), as well as obsessive-compulsive disorder (OCD), and posttraumatic stress disorder (PTSD). Also included are brief discussions of clinically relevant issues in the management of anxiety and related disorders in children and adolescents, women who are pregnant or lactating, and elderly patients, and patients with comorbid conditions. 


\section{Methods}

These guidelines are based on a thorough review of the current literature and were developed by a panel of Canadian experts in anxiety and related disorders through a consensus process. Data on the epidemiology, diagnosis, and treatment (psychological and pharmacological) were obtained through MEDLINE searches of English language citations (1980-2012), using search terms encompassing the specific treatments and specific anxiety and related disorders. These searches were supplemented with data from PsycINFO and manual searches of the bibliographies of efficacy studies, meta-analyses, and review articles. Treatment strategies were rated on strength of evidence for the intervention (Table 1). A clinical recommendation for each intervention was then made, based on global impression of efficacy in clinical trials, effectiveness in clinical practice, and side effects, using a modified version of the periodic health examination guidelines (Table 2).

The guidelines were initiated prior to the introduction of the American Psychiatric Association's (APA) fifth edition of the Diagnostic and Statistical Manual of Mental Disorders (DSM-5) and the committee was sensitive to potential changes to the nosology of anxiety and related disorders and its impact on the guidelines. However, it was agreed that, since the evidence for treatment is based on studies using DSM-IV criteria (or earlier), the introduction of the DSM-5 would not fundamentally alter the evidence and recommendations at this time. Whether using DSM-5 diagnostic criteria for the inclusion patients in clinical trials in the future will have an impact on outcomes, remains to be seen.

The panel of Canadian experts in anxiety and related disorders responsible for the development of these guidelines via consensus process included 10 psychiatrists and seven psychologists who were organized into subpanels based on their expertise in particular anxiety or related disorders as well as in treating specific patient populations. Preliminary treatment recommendations

\section{Table 1 Levels of evidence}

1 Meta-analysis or at least 2 randomized controlled trials (RCTs) that included a placebo condition

2 At least 1 RCT with placebo or active comparison condition

3 Uncontrolled trial with at least 10 subjects

$4 \quad$ Anecdotal reports or expert opinion

Levels of evidence do not assume positive or negative or equivocal results, they merely represent the quality and nature of the studies that have been conducted.

Level 1 and Level 2 evidence refer to treatment studies in which randomized comparisons are available. Recommendations involving epidemiological or risk factors primarily arise from observational studies, hence the highest level of evidence for these is usually Level 3. Recommendations, such as principles of care, reflect consensus opinion based on evidence from various data sources, and therefore are primarily Level 4 evidence.

\section{Table 2 Treatment recommendation summary}

\begin{tabular}{ll}
\hline First-line & $\begin{array}{l}\text { Level } 1 \text { or Level } 2 \text { evidence plus clinical support for } \\
\text { efficacy and safety }\end{array}$ \\
Second-line & $\begin{array}{l}\text { Level } 3 \text { evidence or higher plus clinical support for } \\
\text { efficacy and safety }\end{array}$ \\
Third-line & $\begin{array}{l}\text { Level } 4 \text { evidence or higher plus clinical support for } \\
\text { efficacy and safety }\end{array}$ \\
$\begin{array}{l}\text { Not } \\
\text { recommended }\end{array}$ & Level or Level 2 evidence for lack of efficacy
\end{tabular}

and the evidence upon which they had been based were reviewed at a meeting of the panel in December 2012; subsequently, draft guidelines were prepared by the subpanels which were then circulated to the entire group for consensus ratification during 2013. Preliminary recommendations were also presented to the Canadian psychiatric community for input in September 2012 at the Canadian Psychiatric Association annual conference.

These guidelines are presented in 10 sections, the first of which is this introduction. In the following section, the principles of diagnosis and management of anxiety and related disorders are covered. That section provides an overview of the differential diagnoses associated with anxiety and related disorders in general, discusses issues that affect all anxiety disorders, and presents the general advantages and disadvantages of psychological treatment and pharmacotherapy options. In the subsequent six sections (Sections 3 through 8), the specific diagnosis and management of the individual anxiety and related disorders (panic disorder, specific phobia, SAD, OCD, GAD, and PTSD) are reviewed and recommendations are made for psychological and pharmacological treatments. Section 9 discusses issues that may warrant special attention pertaining to anxiety and related disorders in children and adolescents, pregnant or lactating women, and the elderly. The last section of these guidelines addresses clinical issues that may arise when treating patients with anxiety and related disorders who are also diagnosed with comorbid psychiatric conditions such as major depressive disorder (MDD), bipolar disorder, or other psychoses, and attention deficit/hyperactivity disorder (ADHD), or medical comorbidities, such as pain syndromes, cardiovascular disease, and diabetes/metabolic syndrome.

\section{Principles of diagnosis and management of anxiety and related disorders Epidemiology Prevalence and impact}

Anxiety and related disorders are among the most common mental disorders, with lifetime prevalence rates as high as $31 \%$ [1-5] and 12-month prevalence rates of about $18 \%[3,4]$. Rates for individual disorders vary widely. Women generally have higher prevalence rates 
for most anxiety disorders, compared with men [4,5,9]. Anxiety and related disorders are associated with an increased risk of developing a comorbid major depressive disorder [10-12].

Anxiety and related disorders put a significant burden on patients and their family members [13]. They are associated with substantial functional impairment, which increases as the severity of anxiety [14] or the number of comorbid anxiety disorders increases [7,15]. In addition, studies have demonstrated quality of life impairments in patients with various anxiety and related disorders $[16,17]$. Anxiety has a considerable economic impact on society as well, being associated with greater use of health care services $[5,18]$ and decreased work productivity $[18,19]$.

Importantly, studies report that about $40 \%$ of patients diagnosed with anxiety and related disorder are untreated $[5,7]$.

\section{Suicide risk}

In large surveys, anxiety and related disorders were independently associated with a significant 1.7-2.5 times increased risk of suicide attempts [20-23]; however, data are conflicting as to whether the risk is moderated by gender [20,23]. Increased risk of suicide attempts or completed suicide has been reported for patients with panic disorder, PTSD [20,24], and GAD [24], even in the absence of a comorbid mood disorder. These data indicate that patients with an anxiety disorder warrant explicit evaluation for suicide risk. The presence of a comorbid mood disorder significantly increases the risk of suicidal behavior [22,25].

\section{Initial assessment of patients with anxiety}

The management of patients presenting with anxiety symptoms should initially follow the flow of the five main components outlined in Table 3.

\section{Screen for anxiety and related symptoms}

Anxiety and related disorders are generally characterized by the features of excessive anxiety, fear, worry, and avoidance. While anxiety can be a normal part of everyday life, anxiety disorders are associated with functional impairment; as part of the key diagnostic criteria for anxiety disorders is the requirement that the symptoms cause clinically significant distress or impairment in social, occupational, or other important areas of functioning [26].

\section{Table 3 Overview of the management of anxiety and related disorders}

- Screen for anxiety and related symptoms

- Conduct differential diagnosis (consider severity, impairment, and

comorbidity)

- Identify specific anxiety or related disorder

- Psychological and/or pharmacological treatment

- Perform follow-up
Asking patients if they are feeling nervous, anxious or on edge, or whether they have uncontrollable worry, can be useful to detect anxiety in patients in whom the clinician suspects an anxiety or related disorder [7]. The DSM-5 suggests the questions shown in Table 4 for the identification of anxiety-related symptoms; items scored as mild or greater may warrant further assessment [26]. If anxiety symptoms are endorsed, they should be explored in more detail by including questions about the onset of the anxiety symptoms, associations with life events or trauma, the nature of the anxiety (i.e., worry, avoidance, or obsession), and the impact they have had on the patient's current functioning.

Table 5 presents suggested screening questions for individual anxiety and related disorders, from various validated screening tools [27-30], some of which are freely available online (e.g., http://www.macanxiety.com/ online-anxiety-screening-test).

\section{Conduct differential diagnosis}

The differential diagnosis of anxiety and related disorders should consider whether the anxiety is due to another medical or psychiatric condition, is comorbid with another medical or psychiatric condition, or is medication-induced or drug-related [32].

When a patient presents with excessive or uncontrollable anxiety it is important to identify other potential causes of the symptoms, including direct effects of a substance (e.g., drug abuse or medication) or medical condition (e.g., hyperthyroidism, cardiopulmonary disorders, traumatic brain injury), or another mental disorder [26]. However, since comorbid conditions are common, the presence of some of these other conditions may not preclude the diagnosis of an anxiety or related disorder.

Certain risk factors have been associated with anxiety and related disorders and should increase the clinician's index of suspicion (Table 6) [4,9,33-37]. A family [33] or personal history of mood or anxiety disorders [34,35] is an important predictor of anxiety symptoms. In addition, family history is associated with a more recurrent course, greater impairment, and greater service use [33]. A personal history of stressful life events is also associated the development of anxiety and related disorders [36,37], in particular, childhood abuse [37].

Women generally have higher prevalence rates across all anxiety and related disorders, compared with men $[4,5,9]$. The median of age of onset is very early for some

\section{Table 4 General screening questions}

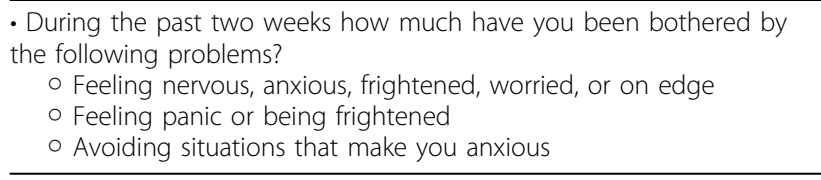

Adapted from reference [26]. 
Panic disorder - MACSCREEN $[29,30]$

- Do you have sudden episodes/spells/attacks of intense fear or discomfort that are unexpected or out of the blue?

If you answered "YES" then continue

- Have you had more than one of these attacks?

- Does the worst part of these attacks usually peak within several minutes?

- Have you ever had one of these attacks and spent the next month or more living in fear of having another attack or worrying about the consequences of the attack?

SAD (Based on Mini-SPIN [28])

- Does fear of embarrassment cause you to avoid doing things or speaking to people?

- Do you avoid activities in which you are the center of attention?

- Is being embarrassed or looking stupid among your worst fears?

GAD [31]

- During the past 4 weeks, have you been bothered by feeling worried, tense, or anxious most of the time?

- Are you frequently tense, irritable, and having trouble sleeping?

OCD - MACSCREEN [29,30]

Obsessions:

- Are you bothered by repeated and unwanted thoughts of any of the following types:

$\circ$ Thoughts of hurting someone else

- Sexual thoughts

- Excessive concern about contamination/germs/disease

- Preoccupation with doubts ("what if" questions) or an inability to make decisions

- Mental rituals (e.g., counting, praying, repeating)

O Other unwanted intrusive thoughts

- If you answered "YES" to any of the above... Do you have trouble resisting these thoughts, images, or impulses when they come into your mind?

Compulsions:

- Do you feel driven to perform certain actions or habits over and over again, or in a certain way, or until it feels just right? Such as:

- Washing, cleaning

$\circ$ Checking (e.g., doors, locks, appliances)

○ Ordering/arranging

$\circ$ Repeating (e.g., counting, touching, praying)

- Hoarding/collecting/saving

- If you answered "YES" to any of the above... Do you have trouble resisting the urge to do these things?

PTSD - MACSCREEN [29,30]

- Have you experienced or seen a life-threatening or traumatic event such as a rape, accident, someone badly hurt or killed, assault, natural or man-made disaster, war, or torture?

If you answered "YES" then continue

- Do you re-experience the event in disturbing (upsetting) ways such as dreams, intrusive memories, flashbacks, or physical reactions to situations that remind you of the event?

phobias and for separation anxiety disorder (seven to 14 years), but later for GAD, panic disorder, and PTSD (24-50 years) $[1,2]$.

Loneliness [38], low education [38], and adverse parenting [39], as well as chronic somatic illnesses, such as cardiovascular disease, diabetes, asthma, and obesity may increase the risk for a lifetime diagnosis of anxiety [34,40]. Comorbid medical and psychiatric disorders Anxiety and related disorders frequently co-occur with other psychiatric disorders [3]. More than half of patients with an anxiety disorder have multiple anxiety disorders $[3,15]$,

Table 6 Common risk factors in patients with anxiety and related disorders

- Family history of anxiety [33]

- Personal history of anxiety or mood disorder [34,35]

- Childhood stressful life events or trauma [36,37]

- Being female $[4,9]$

- Chronic medical illness [34,40]

- Behavioral inhibition [41,42] and almost 30\% will have three or more comorbid anxiety or related disorders [3]. Anxiety is often comorbid with substance use and mood disorders [3,40]. An estimated $52 \%$ of patients with bipolar disorder [43], $60 \%$ of patients with MDD [44], and 47\% of those with ADHD [45] will have a comorbid anxiety or related disorder. Therefore, anxiety disorders should be considered in these patients.

The high frequency of comorbidity must be considered when diagnosing anxiety and related disorders since this can have important implications for diagnosis and treatment [32]. Anxiety disorders comorbid with other anxiety or depressive disorders are associated with poorer treatment outcomes, greater severity and chronicity [46-49], more impaired functioning [46], increased health service use [50], and higher treatment costs [51]. The impact tends to increase with an increasing number of comorbid conditions [46].

Patients with anxiety disorders have a higher prevalence of hypertension and other cardiovascular conditions, gastrointestinal disease, arthritis, thyroid disease, 
respiratory disease, migraine headaches, and allergic conditions compared to those without anxiety disorders $[16,52]$. Comorbid anxiety and related disorders have a significant impact on quality of life (QoL) in patients with medical conditions [52].

Baseline assessment Baseline assessment should include a review of systems, prescribed medications, over-thecounter agents, alcohol use, caffeine intake, and illicit drug use, in addition to evaluation of the anxiety symptoms and functioning [32]. Table 7 lists potential investigations that can be considered based on an individual patient's presentation and specific symptoms (e.g., dizziness or tachycardia). Ideally, a physical examination and baseline laboratory investigations should be performed before pharmacotherapy is initiated, with repeat assessments according to best practice guidelines [32]. Patients with anxiety and related disorders should be monitored initially every one to two weeks and then every four weeks for weight changes and adverse effects of medications, as this is a major factor contributing to discontinuation of medication.

Closer monitoring may be required in children younger than 10 years of age, older or medically ill patients, patients on medications associated with metabolic changes, and those on multiple medications [32].

\section{Identify specific anxiety or related disorder}

The fifth edition of the Diagnostic and Statistical Manual of Mental Disorders (DSM-5) has been finalized by the American Psychiatric Association (APA) [26]. The new DSM-5 provides diagnostic criteria for psychiatric disorders based on scientific reviews of the literature, field trial data, internal evaluations, public comments, and a final review by APA's Board of Trustees.

The "anxiety disorders" chapter now includes panic disorder, agoraphobia, GAD, selective mutism, separation anxiety disorder, SAD (social phobia), specific phobia, substance/medication-induced anxiety disorder, as well as anxiety disorder due to another medical condition or not elsewhere classified. OCD and PTSD have been moved to separate chapters on obsessive-compulsive and

\section{Table 7 Considerations for baseline laboratory investigations (as needed based on patient's presenting symptoms)}

\begin{tabular}{|c|c|}
\hline Basic lab tests & \\
\hline - Complete blood count & - Fasting glucose \\
\hline - Fasting lipid profile (TC, vLDL, LDL, HDL, TG) & $\begin{array}{l}\text { - Thyroid-stimulating } \\
\text { hormone }\end{array}$ \\
\hline - Electrolytes & - Liver enzymes \\
\hline If warranted & \\
\hline - Urine toxicology for substance use & \\
\hline
\end{tabular}

Adapted from references $[32,53]$. $\mathrm{HDL}=$ high density lipoprotein; $\mathrm{LDL}=$ low density lipoprotein; $\mathrm{TC}=$ total cholesterol; $\mathrm{TG}=$ triglyceride; $\mathrm{VLD}=$ very low density lipoprotein. related disorders and trauma- and stressor-related disorders, respectively [26].

Table 8 provides a brief summary of the key DSM-5 diagnostic features of the anxiety and related disorders that are included in these guidelines [26]. While the DSM-5 is the most up-to-date diagnostic criteria, it is important to note that the evidence for treatment is based on studies using DSM-IV criteria (or earlier) for inclusion of patients. However, most of the diagnostic criteria have not changed substantially (see Sections 3-9 for more information on diagnosis); the exception being agoraphobia, which is now designated as a separate diagnosis.

Specific individual anxiety and related disorders should be diagnosed with the DSM-5 criteria in the sections devoted to each anxiety disorder. An accurate diagnosis is important to help guide treatment.

\section{Psychological and pharmacological treatment}

Treatment options for anxiety and related disorders include psychological and pharmacological treatments. All patients should receive education about their disorder, efficacy (including expected time to onset of therapeutic effects) and tolerability of treatment choices, aggravating factors, and signs of relapse [32]. Information on self-help materials such as books or websites may also be helpful.

The choice of psychological or pharmacological treatment depends on factors such as patient preference and motivation, ability of the patient to engage in the treatment, severity of illness, clinicians' skills and experience, availability of psychological treatments, patient's prior response to treatment, and the presence of comorbid medical or psychiatric disorders [32].

A brief overview of psychological and pharmacological treatments is provided below, with more specific recommendations in the individual sections for each anxiety and related disorder.

Overview of psychological treatment Psychological treatments play an important role in the management of anxiety and related disorders. Regardless of whether formal psychological treatment is undertaken, patients should receive education and be encouraged to face their fears. Meta-analyses have demonstrated the efficacy of psychological treatments in group and individual formats in patients with panic disorder [54-56], specific phobia [57], SAD [58,59], OCD [60-63], GAD [55,64,65], or PTSD [66-69], particularly exposure-based and other cognitive behavioral therapy (CBT) protocols $[70,71]$, as well as mindfulness-based cognitive therapy (MBCT) [72]. When choosing psychological treatments for individual patients, the forms of therapy that have been most thoroughly evaluated in the particular anxiety or related disorder should be used first.

CBT is not a single approach to treatment, but rather a process that focuses on addressing the factors that 
Table 8 Key features of specific anxiety and related disorders

\begin{tabular}{|c|c|}
\hline Disorder & Key features \\
\hline Panic disorder & $\begin{array}{l}\text { - Recurrent unexpected panic attacks, in the absence of triggers } \\
\text { - Persistent concern about additional panic attacks and/or maladaptive change in behavior related to the attacks }\end{array}$ \\
\hline Agoraphobia & $\begin{array}{l}\text { - Marked, unreasonable fear or anxiety about a situation } \\
\text { - Active avoidance of feared situation due to thoughts that escape might be difficult or help unavailable if panic-like } \\
\text { symptoms occur }\end{array}$ \\
\hline Specific phobia & $\begin{array}{l}\text { - Marked, unreasonable fear or anxiety about a specific object or situation, which is actively avoided (e.g., flying, } \\
\text { heights, animals, receiving an injection, seeing blood) }\end{array}$ \\
\hline Social anxiety disorder (SAD) & $\begin{array}{l}\text { - Marked, excessive or unrealistic fear or anxiety about social situations in which there is possible exposure to } \\
\text { scrutiny by others } \\
\text { - Active avoidance of feared situation }\end{array}$ \\
\hline $\begin{array}{l}\text { Generalized anxiety disorder } \\
\text { (GAD) }\end{array}$ & $\begin{array}{l}\text { - Excessive, difficult to control anxiety and worry (apprehensive expectation) about multiple events or activities (e.g., } \\
\text { school/work difficulties) } \\
\text { - Accompanied by symptoms such as restlessness/feeling on edge or muscle tension }\end{array}$ \\
\hline $\begin{array}{l}\text { Obsessive-compulsive } \\
\text { disorder (OCD) }\end{array}$ & $\begin{array}{l}\text { - Obsessions: recurrent and persistent thoughts, urges, or images that are experienced as intrusive and unwanted } \\
\text { and that cause marked anxiety or distress } \\
\text { - Compulsions: repetitive behaviors (e.g., hand washing) or mental acts (e.g., counting) that the individual feels driven } \\
\text { to perform to reduce the anxiety generated by the obsessions }\end{array}$ \\
\hline $\begin{array}{l}\text { Posttraumatic stress disorder } \\
\text { (PTSD) }\end{array}$ & $\begin{array}{l}\text { - Exposure to actual or threatened death, serious injury, or sexual violation } \\
\text { - Intrusion symptoms (e.g., distressing memories or dreams, flashbacks, intense distress) and avoidance of stimuli } \\
\text { associated with the event } \\
\text { - Negative alterations in cognitions and mood (e.g., negative beliefs and emotions, detachment), as well as marked } \\
\text { alterations in arousal and reactivity (e.g., irritable behavior, hypervigilance) }\end{array}$ \\
\hline
\end{tabular}

Adapted from reference [26].

caused and maintain the individual patient's anxiety symptoms [73]. Some of the core components of CBT are shown in Table 9 [73].

CBT can be effectively delivered as individual or group therapy for most anxiety and related disorders. In addition, a variety of self-directed or minimal intervention formats (e.g., bibliotherapy/self-help books, or internet/ computer-based programs with or without minimal therapist contact) have demonstrated significant improvements in anxiety symptoms [74-79]. Meta-analyses have also shown that exposure therapy can be effectively administered in a virtual reality format $[80,81]$. These strategies may be particularly useful in cases where real-life exposure is difficult due to inconvenience, expense, or patient reluctance.
Psychotherapy and pharmacotherapy generally demonstrate about equivalent efficacy for the treatment of most anxiety and related disorders $[71,82]$. Results with combination therapy vary for the different anxiety disorders, and results have been conflicting $[82,83]$ (see Sections 39 for evidence and references regarding combination therapy). Therefore, current evidence does not support the routine combination of CBT and pharmacotherapy as initial treatment. However, when patients do not benefit from CBT or have a limited response, a trial of pharmacotherapy is advisable. Similarly, patients who show limited benefit from pharmacotherapy may benefit from CBT. All patients being treated with pharmacotherapy should be instructed to gradually face their fears (exposure to decrease avoidance).

Table 9 Components of cognitive behavioral interventions

\begin{tabular}{|c|c|}
\hline Exposure & $\begin{array}{l}\text { - Encourage patients to face fears } \\
\text { - Patients learn corrective information through experience } \\
\text { - Extinction of fear occurs through repeated exposure } \\
\text { - Successful coping enhances self-efficacy }\end{array}$ \\
\hline $\begin{array}{l}\text { Safety response } \\
\text { inhibition }\end{array}$ & $\begin{array}{l}\text { - Patients restrict their usual anxiety-reducing behaviors (e.g., escape, need for reassurance) } \\
\text { - Decreases negative reinforcement } \\
\text { - Coping with anxiety without using anxiety-reducing behavior enhances self-efficacy }\end{array}$ \\
\hline Cognitive strategies & $\begin{array}{l}\text { - Cognitive restructuring, behavioral experiments, and related strategies target patients' exaggerated perception of danger } \\
\text { (e.g., fear of negative evaluation in SAD) } \\
\text { - Provides corrective information regarding the level of threat } \\
\text { - Can also target self-efficacy beliefs }\end{array}$ \\
\hline Arousal management & - Relaxation and breathing control skills can help patient control increased anxiety levels \\
\hline $\begin{array}{l}\text { Surrender of safety } \\
\text { signals }\end{array}$ & $\begin{array}{l}\text { - Patient relinquishes safety signals (e.g., presence of a companion, knowledge of the location of the nearest toilet) } \\
\text { - Patients learn adaptive self-efficacy beliefs }\end{array}$ \\
\hline
\end{tabular}

Adapted from reference [73]. 
Overview of pharmacological treatment This section provides a general overview of some of the commonly recommended pharmacological agents. Evidence and recommendations for specific medications are described in the individual sections for each of the anxiety and related disorders.

Table 10 shows medications that have Health Canada approved indications for use in different anxiety and related disorders [84], and dosing suggestions are shown in Additional file 1. Various antidepressants including selective serotonin reuptake inhibitors (SSRIs), serotonin norepinephrine reuptake inhibitors (SNRIs), noradrenergic and specific serotonergic antidepressants (NaSSAs), tricyclic antidepressants (TCAs), monoamine oxidase inhibitors (MAOIs), and reversible inhibitors of monoamine oxidase A (RIMAs) have demonstrated some efficacy in the treatment of anxiety and related disorders (see Sections 3-9 for evidence and references). SSRIs and SNRIs are usually preferred as initial treatments, since they are generally safer and better tolerated than TCAs or MAOIs [32].

Benzodiazepines may be useful as adjunctive therapy early in treatment, particularly for acute anxiety or agitation, to help patients in times of acute crises, or while waiting for onset of adequate efficacy of SSRIs or other antidepressants [32]. Due to concerns about possible dependency, sedation, cognitive impairment, and other side effects, benzodiazepines should usually be restricted to short-term use, and generally dosed regularly rather than as-needed [32].

Several anticonvulsants and atypical antipsychotics have demonstrated efficacy in some anxiety and related disorders, but for various reasons, including side effects, as well as limited randomized controlled trial (RCT) data and clinical experience, these agents are generally recommended as second-line, third-line, or adjunctive therapies (see Sections 3-9 for evidence and references).

The choice of medication should take into consideration the evidence for its efficacy and safety/tolerability for the treatment of the specific anxiety and related disorder, as well as for any comorbid conditions the patient might have, in both acute and long-term use.

Safety and side effects Antidepressants: The most common side effects seen with SSRIs and SNRIs include headache, irritability, gastrointestinal complaints, insomnia, sexual dysfunction, weight gain, increased anxiety, drowsiness, and tremor [85-88]. Patients report that the most common bothersome side effects are sexual dysfunction, drowsiness, fatigue, and weight gain $[87,88]$. Most side effects occur early and transiently during the first two weeks of treatment, but others, such as sexual dysfunction and weight gain, may persist for the duration of treatment $[85,87,89]$.

Use of SSRIs or SNRIs has been associated with an increased risk of upper gastrointestinal bleeding,

Table 10 Medications with Health Canada-approved indications for anxiety and related disorders

\begin{tabular}{|c|c|c|c|c|c|c|}
\hline & $\begin{array}{l}\text { Anxiety } \\
\text { disorders }\end{array}$ & $\begin{array}{c}\text { Panic } \\
\text { disorder }\end{array}$ & $\begin{array}{l}\text { Social anxiety } \\
\text { disorder }\end{array}$ & $\begin{array}{c}\text { Obsessive-compulsive } \\
\text { disorder }\end{array}$ & $\begin{array}{c}\text { Generalized anxiety } \\
\text { disorder }\end{array}$ & $\begin{array}{c}\text { Posttraumatic stress } \\
\text { disorder }\end{array}$ \\
\hline \multicolumn{7}{|l|}{ ANTIDEPRESSANTS } \\
\hline \multicolumn{7}{|l|}{ SSRIs } \\
\hline Escitalopram (Cipralex ${ }^{\circledR}$ ) & & & & $x$ & $x$ & \\
\hline Fluoxetine $\left(\operatorname{Prozac}^{\mathbb{R}}\right)$ & & & & $x$ & & \\
\hline Fluvoxamine (Luvox $\left.{ }^{\circledR}\right)$ & & & & $x$ & & \\
\hline$\left.\overline{\text { Paroxetine }(\text { Paxil }}{ }^{\mathbb{R}}\right)$ & & $x$ & $x$ & $x$ & $x$ & $x$ \\
\hline Paroxetine CR (Paxil $\left.{ }^{\mathbb{R}} \mathrm{CR}\right)$ & & $x$ & $x$ & & & \\
\hline Sertraline $\left(\right.$ Zoloft $\left.^{\circledR}\right)$ & & $x$ & & $x$ & & \\
\hline \multicolumn{7}{|l|}{ TCAs } \\
\hline Clomipramine & & & & $x$ & & \\
\hline \multicolumn{7}{|l|}{ Other antidepressants } \\
\hline $\begin{array}{l}\text { Venlafaxine XR (Effexor }{ }^{\circledR} \\
\text { XR) }\end{array}$ & & $x$ & $x$ & & $x$ & \\
\hline Duloxetine $\left(\right.$ Cymbalta $\left.{ }^{\circledR}\right)$ & & & & & $x$ & \\
\hline \multicolumn{7}{|l|}{ AZAPIRONES } \\
\hline $\begin{array}{l}\text { Buspirone }\left(\text { BuSpar }^{\mathbb{R}},\right. \\
\text { Buspirex }\end{array}$ & & & & & $x$ & \\
\hline BENZODIAZEPINES* & $x$ & & & & & \\
\hline
\end{tabular}


particularly when used in combination with nonsteroidal anti-inflammatory drugs (NSAIDs) [90,91]. SSRI use has also been associated with low bone mineral density $[92,93]$, as well as an increased risk of fractures [94] and hyponatremia [95].

Abrupt discontinuation of SSRIs or SNRIs can lead to a discontinuation syndrome with gastrointestinal, psychiatric, vasomotor, and other symptoms $[85,96]$.

Health Canada and the US Food and Drug Administration (FDA) require antidepressants to include a warning regarding an increased risk of suicidal ideation and behavior in children and adolescents [97,98]. The increased risk of suicidal behavior reported in pediatric patients [99] does not appear to be seen in adults, and may in fact be decreased [99,100]. Careful monitoring for evidence of self-harming or suicidal thoughts or behaviors is important in both adult and pediatric patients.

SSRIs and SNRIs are generally better tolerated and safer than TCAs and MAOIs, having less anticholinergic effects, toxicity, lethality, and psychomotor or cognitive impairment $[85,101]$. MAOIs are generally reserved for second- or third-line treatment because of side effects, drug interactions, and dietary restrictions [32].

Anxiolytics: The most common side effects associated with benzodiazepines include primarily sedation, fatigue, ataxia, slurred speech, memory impairment, and weakness [85]. Benzodiazepines are associated with withdrawal reactions, rebound, and dependence, with the risk being greater with short- and intermediate-acting compared to long-acting agents [102]. These agents should be used with caution in patients with SUDs $[85,103]$. Older patients (generally over 65 years of age) may be at high risk for falls and fractures due to psychomotor impairment associated with benzodiazepines [104,105]. Cognitive impairment has been reported [106], some of which may persist after cessation of therapy [107]. In particular, memory impairment has been associated with high-dose or high-potency benzodiazepines, particularly in older people [102,107].

Reported side effects of azapirones (buspirone) include dizziness, drowsiness, and nausea [32,108].

Atypical antipsychotics: Atypical antipsychotics are associated to varying degrees with weight gain, diabetes, and other metabolic side effects, including alterations in glucose and lipid levels [109-116]. Metabolic disturbances generally appear to be higher with olanzapine, intermediate with risperidone and quetiapine, and lower with aripiprazole, asenapine, lurasidone, and ziprasidone [109-114].

Atypical antipsychotics have varying sedative effects, with quetiapine, clozapine, asenapine, and olanzapine generally causing more sedation than ziprasidone, risperidone, lurasidone, or aripiprazole [111,115]. Data on cognitive effects are conflicting, with some studies suggesting improvements [111], while other data suggest greater cognitive dysfunction in patients using, versus those not using, antipsychotics [117].

Because of the risks of diabetes and weight gain, and the fact that there is limited RCT evidence of the efficacy of these agents in anxiety and related disorders, atypical antipsychotics are generally recommended as second-line, third-line, or adjunctive therapies (see Sections 3-9 for evidence and references).

Anticonvulsants: Anticonvulsants are associated with gastrointestinal side effects, somnolence, weight gain, tremor, as well as dermatologic and hematologic side effects $[111,118]$. In addition, several anticonvulsants have a potential risk of serious rash, erythema multiforme, Stevens-Johnson syndrome, or toxic epidermal necrolysis [111]. Regular monitoring of serum medication levels and liver function is required for patients on divalproex $[84,111]$.

\section{Follow-up}

Anxiety and related disorders are often chronic and a systematic approach to treatment should include patient education, assessment of comorbidities, and evidencebased pharmacological and psychological interventions with adequate monitoring and duration. Pharmacological treatment is often associated with a delay of about two to eight weeks in onset of symptom relief, with full response taking up to 12 weeks or more. Longer-term therapy has been associated with continued symptomatic improvement and the prevention of relapse, and therapy should be continued for at least 12-24 months for most patients [32].

Medication should be initiated at low doses and titrated to the recommended dosage range at one- to two-week intervals over four to six weeks. Once the therapeutic range has been achieved, improvement is usually seen over the next four to eight weeks. Followup should occur at two-week intervals for the first six weeks and monthly thereafter [32].

For a patient undergoing psychotherapy, the treatment schedule is structured around weekly contact with a therapist for about 12-20 weeks, although shorter protocols and minimal intervention programs have also proven effective (see Sections 3-9 for evidence and references). A followup appointment four weeks later and then every two to three months is usually sufficient [32].

Assessing response to treatment Therapy should seek to improve symptoms and distress. The optimal goal is full remission of symptoms and return to a premorbid level of functioning $[32,85]$. However, goals may need to be individualized for some patients with disorders that have been present since childhood as they may never have had adequate premorbid functioning. A response to therapy is often defined as a percentage reduction in symptoms (usually 25-50\%) on an appropriate scale. Remission is often defined as loss of diagnostic status, a pre-specified low score on an appropriate disorder-specific scale, and 
no functional impairment in fully recovered patients as measured by a scale such as the Sheehan Disability Scale or SF-36 [32,119,120].

Objective scales can be used to help assess a patient's progress. The Clinical Global Impression (CGI) scale is brief, comprehensive, and can easily be used at each appointment to assess improvement. The clinician-rated Hamilton Anxiety Rating Scale (HARS) can assess anxiety symptoms in general and is often used in clinical trials but is less practical in clinical practice. A variety of self-report and clinician-rated scales are available to assess the specific anxiety or related disorder.

\section{Panic disorder and agoraphobia Epidemiology}

The lifetime and 12-month prevalence of panic disorder have been estimated at $4.7-5.1 \%$ and $2.1-2.8 \%$, respectively $[121,122]$. The estimated prevalence of panic attacks is considerably greater at $28.3 \%$ (lifetime) and $6.4-11.2 \%$ (12-month) $[121,123]$. Youth with panic attacks (which often do not meet diagnostic criteria for panic disorder) will frequently have or develop other psychiatric disorders including mood disorders (bipolar disorder and MDD), other anxiety or related disorders, SUDs, eating disorders, psychotic disorders, and personality disorders $[122,124,125]$. Annually, $8-10 \%$ of the general public will have a panic attack without ever developing any identifiable psychopathology [126]. About $40-70 \%$ of patients with panic disorder experience nocturnal panic (waking from sleep in a state of panic) [127]. Rates of 12-month and lifetime agoraphobia (without panic) are quite low, at $0.8 \%$ and $1.4 \%$, respectively $[2,3]$.

The risk of panic disorder and agoraphobia is higher in women than men, and patients who are middle-aged, widowed/divorced, and those of low income [122]. In the Canadian Community Health Survey 1.2 (CCHS 1.2) there were no differences in the rates of panic disorder or agoraphobia in urban versus rural settings [128].

Panic disorder has a negative impact on both psychological and physical functioning, and puts a substantial burden on the patient's family [13]. Patients with panic disorder have more QoL impairment and dissatisfaction $[16,17]$, greater likelihood of suicide attempts [20], and increased cognitive and emotional dysfunction [129-133] compared to healthy controls. Panic disorder is also associated with substantial societal costs [134], both in terms of health care utilization [135] and loss of workplace productivity [136]. In a 2012 survey, panic disorder conferred a substantial rate of work absenteeism (mean: 36.0 days/year) [136].

\section{Comorbidity}

Patients with panic disorder, or those experiencing panic attacks, have significantly increased odds of being diagnosed with a comorbid disorder, including another anxiety or related disorder, mood disorder, impulse-control disorder, or SUD $[121,137]$. MDD is very common, occurring in an estimated $35-40 \%$ of patients with panic disorder [121]. Panic disorder also frequently co-occurs with agoraphobia [138].

Panic disorder is more prevalent in patients with medical conditions, including thyroid disease, cancer, chronic pain, cardiac disease, irritable bowel syndrome, migraine, as well as allergic and respiratory diseases compared with the general population [85,139-141]. The presence of medical comorbidity is associated with greater severity of panic disorder symptoms and disability $[140,142]$.

\section{Diagnosis}

For a diagnosis of panic disorder, a patient must have had recurrent, unexpected panic attacks (Table 11), followed by at least one month of persistent concern or worry about further attacks or their consequences, or a significant maladaptive behavioral change related to attacks (Table 12) [26].

A panic attack continues to be considered a noncodable event in the DSM-5, with only minor revisions, including removal of the "10-minute" window, changing "hot flushes" to "heat sensations," and the re-ordering of the list of symptoms to increase clinical utility [26,143].

Compared to the DSM-IV-TR [144], changes to the diagnostic criteria for panic disorder largely consisted of minor phrasing changes to improve clinical utility, with the most substantial change being the title of the disorder $[26,143]$. The DSM-5 now lists agoraphobia (anxiety about having a panic attack in certain situations, which are avoided or endured with marked distress) as a separate codable disorder, whereas previously panic disorder could be diagnosed as "panic disorder with agoraphobia" or "panic disorder without agoraphobia" [26,145].

For a diagnosis of agoraphobia, a patient must have intense fear about at least two different types of

\section{Table 11 DSM-5 criteria for panic attacks}

- An abrupt surge of intense fear or intense discomfort that reaches a peak within minutes, and includes $\geq 4$ of the following symptoms:

(1) Palpitations, pounding heart, or accelerated heart rate

(2) Sweating

(3) Trembling or shaking

(4) Sensations of shortness of breath or smothering

(5) Feelings of choking

(6) Chest pain or discomfort

(7) Nausea or abdominal distress

(8) Feeling dizzy, unsteady, light-headed, or faint

(9) Chills or heat sensations

(10) Paresthesias (numbness or tingling sensations)

(11) Derealization (feelings of unreality) or depersonalization (being detached from oneself)

(12) Fear of losing control or going crazy

(13) Fear of dying

Adapted from reference [26]. 


\section{Table 12 DSM-5 diagnosis of panic disorder}

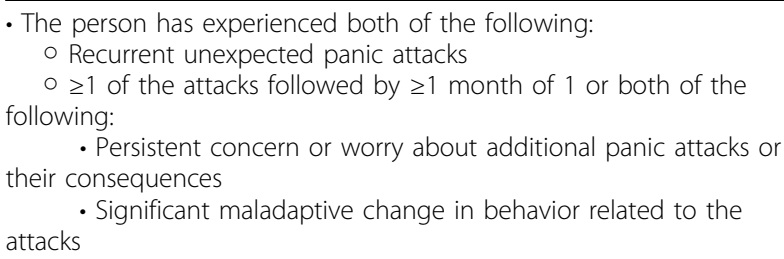

Adapted from DSM-5 [26].

situations, with the fear resulting from thoughts that escape may be difficult or help may be unavailable if panic-like symptoms occur (Table 13$)[26,145]$. The situations provoke anxiety and are avoided or endured with intense fear or anxiety, or may require that a companion be present. The resultant fear or anxiety is out of proportion to any actual danger from the situation, causes substantial functional impairment, and usually lasts for six months or longer [26].

While the most up-to-date DSM-5 diagnostic criteria are presented here, the treatment data described within this section are based on studies involving patients meeting DSM-IV panic criteria (or older).

Establishing the context in which panic attacks occur, and whether there is any prior history of recurrent, unexpected panic attacks, is important for accurate diagnosis. Panic attacks frequently occur in other psychiatric disorders (e.g., MDD, PTSD), and medical conditions (e.g., cardiac, respiratory), and the DSM-5 has identified panic attacks as a specifier to be used in the absence of a diagnosable panic disorder [85]. Another disorder may better account for the panic attacks; for example, panic attacks in social situations may be SAD, those related to defined phobic objects or situations may be specific phobia, those related to reminders of traumatic events

\section{Table 13 DSM-5 diagnosis of agoraphobia}

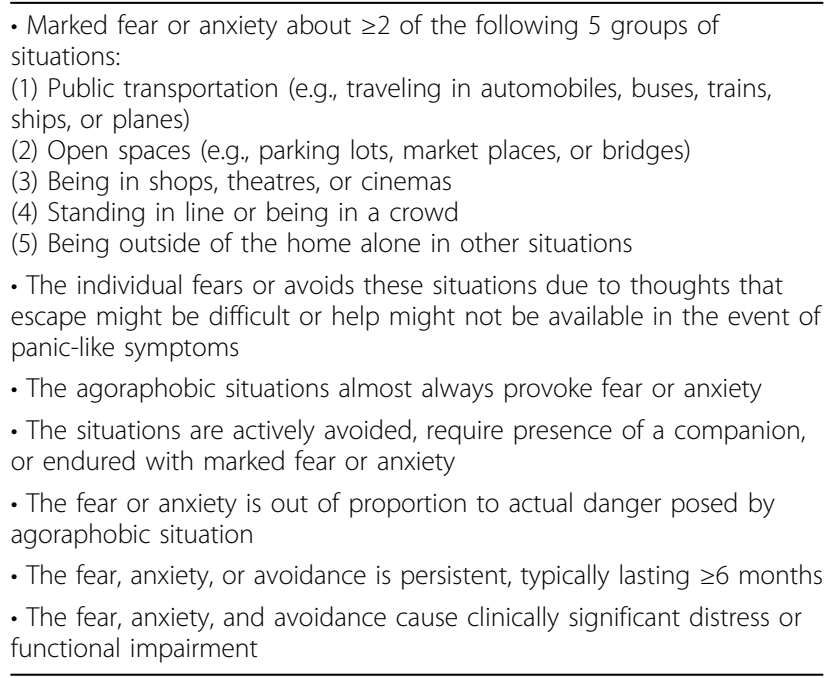

Adapted from DSM-5 [26]. may be PTSD $[26,85]$, and those related to being kidnapped by extraterrestrials may be schizophrenia [26] Some medical conditions that can be associated with panic symptoms include hyper- or hypothyroidism, hypoglycemia, seizure disorders, and cardiac conditions $[26,85]$. Panic attacks may also be associated with intoxication or withdrawal from drugs of abuse, medications such as decongestants, stimulants, or beta-adrenergic agonist inhalers, or caffeine [85].

\section{Psychological treatment}

CBT has been extensively studied, and is an efficacious psychological treatment for panic disorder (Level 1) $[56,70,146,147]$. In fact, CBT was significantly favored over medications for the treatment of panic disorder in a meta-analysis [71]. In a meta-analysis of 42 studies, exposure and combinations of exposure, cognitive restructuring and other CBT techniques had the most consistent evidence of efficacy for the treatment of panic disorder [56]. Strategies that included exposure were the most effective for panic measures. For measures of agoraphobia, combined strategies were more effective than single techniques, which did not result in significant improvements. Factors that improved the effectiveness of treatments were the inclusion of homework and a follow-up program [56]. Another meta-analysis also found that CBT that included interoceptive exposure was superior to relaxation therapy for panic symptoms [55]. CBT can be effectively delivered in both individual and group settings $[56,148,149]$. Conducting exposure in virtual reality appears to be effective when used as part of a CBT protocol [150-154].

Minimal intervention formats, such as self-help books (bibliotherapy) [75,76,155-158], treatment via telephone/ videoconferencing [75,159-161], and internet-based CBT (ICBT) [75,79,162-169] have been shown to be more effective than wait-list or relaxation controls, as effective as face-to-face CBT, and may be cost-effective options particularly for agoraphobic patients who are unwilling or unable to attend a clinic. When using bibliotherapy, providing information all at one time was as effective as pacing [157], and therapist support does not appear to be essential $[75,158]$. Most ICBT programs have some therapist contact by either telephone or email, and once weekly contact appeared to be as effective as more frequent contact [168].

CBT panic disorder protocols usually involve 12-14 weekly sessions, but briefer strategies of six to seven sessions have been shown to be as effective $[148,149,170]$. In addition, compressing the duration of therapy by administering 13 sessions over three weeks has also been shown to be as effective as traditional weekly CBT [171]. Patients with higher baseline severity, disability, or comorbidity may have better outcomes with standard 
CBT [172]. CBT programs sometimes include one or more follow-up or "booster" sessions $[170,173]$.

Predictors of decreased response to CBT were severity of panic disorder, strength of blood/injury fears, earlier age of initial onset of panic symptoms, comorbid social anxieties, and degree of agoraphobic avoidance [174,175]. Changes in symptoms are preceded by changes in beliefs during therapy [176], and change in beliefs and avoidance behaviors are considered key process variables $[170,176]$.

Eye movement desensitization and reprocessing (EMDR) does not appear to offer advantages over the same strategy without the eye movement component for the treatment of panic disorder $[177,178]$.

\section{Combined psychological and pharmacological treatment}

A meta-analysis of 21 trials found that combination psychotherapy and pharmacotherapy with antidepressants was superior to CBT or pharmacotherapy alone during the acute treatment phase and while medication was continued $[179,180]$. After termination of treatment, combined therapy was more effective than pharmacotherapy alone and was as effective as psychotherapy $[179,180]$. Prior meta-analyses have reported similar findings $[54,146,181]$, suggesting that CBT alone or CBT combined with pharmacotherapy should be considered as first-line treatment.

A meta-analysis of the combination of psychotherapy and benzodiazepines included only three trials, and found no benefit to combination therapy compared with psychotherapy or medication alone [182]. The follow-up data suggested that the combination might be inferior to behavior therapy alone [182].

Adding self-administered CBT to SSRI therapy did not result in significant improvements overall, but patients did report a significantly greater rate of decline in fear of bodily sensations compared to medication alone [183]. Early results suggest a benefit of MBCT as an adjunct to pharmacotherapy in relieving anxiety and depressive symptoms in patients with panic disorder [184,185].

Providing CBT sessions around the time of medication discontinuation was associated with a lower relapse rate during follow-up among patients treated with antidepressants [186]. In addition, CBT has been shown to be helpful in facilitating benzodiazepine discontinuation $[187,188]$.

A cost-effectiveness study found that combined CBT and pharmacotherapy was associated with a robust clinical improvement compared to usual care, with only a moderate increase in costs [189].

In a RCT, buspirone enhanced the effects of CBT in the short-term, but had no significant benefit over CBT alone during long-term follow-up [190].

Data on the efficacy of $\mathrm{d}$-cycloserine as an adjunct to CBT are conflicting, with one study suggesting significant benefits at posttreatment and one-month follow-up
[191], while another found an acceleration of symptom reduction in severely ill patients but no significant improvement in outcomes overall [192] compared to CBT plus placebo. Another compound acting at the $N$-methyl-D-aspartate (NMDA) receptor, Org 25935, demonstrated no benefit over placebo in augmenting CBT for panic disorder [193].

\section{Long-term effects of psychological treatment}

In naturalistic long-term follow-up studies, the benefits of CBT were maintained for up to three years $[148,169$, 170,188]. At two-year follow-up, individual, group, and brief CBT were associated with lower relapse rates compared to the wait-list control [148]. A long-term follow-up study of patients who had become panic-free with exposure therapy found that $93 \%$ remained in remission after two years and $62 \%$ after 10 years [194].

A meta-analysis found that at six to 24 months followup, remission/response rates with the combination of psychotherapy and antidepressants continued to be superior to antidepressants alone, or to psychotherapy as long as therapy was continued $[179,180]$.

\section{Pharmacological treatment}

The management of patients with panic disorder should follow the principles discussed in Section 2. Pharmacological interventions that have good evidence for efficacy in treating panic disorder include SSRIs, TCAs, and other antidepressants, as well as benzodiazepines. Treatments that have been investigated for use in panic disorder have been assessed according to the criteria for strength of evidence (Tables 1 and 2) and are summarized in Tables 14 and 15.

\section{First-line agents}

SSRIs: Evidence from meta-analyses [195-197] and RCTs supports the use of the SSRIs citalopram [198-200], fluoxetine [201-204], fluvoxamine [195,205-210], paroxetine [211-219], and sertraline [183,220,221,223,224] (all Level 1), as well as escitalopram [198] and paroxetine controlled-release (CR) [225] (both Level 2) for the treatment of panic disorder. In meta-analyses, SSRIs demonstrated significant improvements in panic symptoms, agoraphobic avoidance, depressive symptomatology, and general anxiety [195-197,226]. Effect sizes for SSRIs and TCAs are similar [195,196], although dropout rates may be lower with SSRIs [195].

SNRIs: Venlafaxine extended-release (XR) has been shown to be useful in reducing the severity of panic disorder symptoms in RCTs (Level 1) [215,216,227-229]. Two studies found significantly greater rates of panicfree patients compared with placebo [215,216] while two did not [228,229].

\section{Second-line agents}

TCAs: There is good evidence from RCTs to support the use of the TCAs clomipramine [199,211,213,232,233] 
Table 14 Strength of evidence for pharmacotherapy for panic disorder

\begin{tabular}{|c|c|c|c|}
\hline Agent & Level of evidence & Agent & Level of evidence \\
\hline \multicolumn{4}{|l|}{ Antidepressants } \\
\hline SSRIs & & TCAs & \\
\hline Citalopram [198-200] & 1 & Clomipramine $[199,211,213,232,233]$ & 1 \\
\hline Fluoxetine [201-204] & 1 & Imipramine $[207,224,233-240]$ & 1 \\
\hline Fluvoxamine [195,205-210] & 1 & MAOls and RIMAs & \\
\hline Paroxetine [211-219] & 1 & Phenelzine [240] & 2 \\
\hline Sertraline [183,220-224] & 1 & Moclobemide $[204,232,241,242]$ & $1^{*}$ \\
\hline Escitalopram [198] & 2 & Tranylcypromine [243] & 3 \\
\hline Paroxetine CR [225] & 2 & Other antidepressants & \\
\hline SNRIs & & Reboxetine $[200,219,244]$ & 1 \\
\hline Venlafaxine XR [215,216,227-229] & 1 & Mirtazapine $[203,245,246]$ & 2 \\
\hline Duloxetine [230] & 3 & Bupropion SR $[247,248]$ & $3^{*}$ \\
\hline Milnacipran [231] & 3 & & \\
\hline \multicolumn{4}{|l|}{ Other therapies } \\
\hline Anxiolytics & & Atypical antipsychotics & \\
\hline Benzodiazepines & & Risperidone $[217,267]$ & 2 \\
\hline Alprazolam [234,249-254] & 1 & Olanzapine [268] & 3 \\
\hline Clonazepam [218,250,255-258] & 1 & Quetiapine [267] & 3 \\
\hline Lorazepam $[251,259,260]$ & 1 & Adjunctive aripiprazole [269] & 3 \\
\hline Diazepam [261-263] & 1 & Adjunctive olanzapine [270] & 3 \\
\hline Adjunctive clonazepam $[264,265]$ & 1 & Adjunctive risperidone [271] & 3 \\
\hline Adjunctive alprazolam ODT [266] & 3 & Anticonvulsants & \\
\hline Other treatments & & Divalproex [272-275] & 3 \\
\hline Buspirone $[254,282]$ & 1 (-ve) & Levetiracetam [276] & 3 \\
\hline Trazodone [283] & $2(-\mathrm{ve})$ & Gabapentin [277] & $2(-v e)^{\dagger}$ \\
\hline Propranolol [262,284,285] & $2(-v e)$ & Tiagabine $[278,279]$ & $2(-v e)$ \\
\hline \multirow[t]{2}{*}{ Adjunctive pindolol [286] } & 2 & Carbamazepine [280] & $3(-v e)$ \\
\hline & & Adjunctive divalproex [281] & 3 \\
\hline
\end{tabular}

${ }^{*}$ Conflicting data. ${ }^{\dagger}$ No significant superiority over placebo in overall population, but significant benefits in subgroup of more severely ill patients. CR = controlled release $\mathrm{MAOI}=$ monoamine oxidase inhibitor; $\mathrm{ODT}=$ orally disintegrating tablets; $\mathrm{RIMA}=$ reversible inhibitor of monoamine oxidase $\mathrm{A}$; $\mathrm{SNRI}=$ serotoninnorepinephrine reuptake inhibitor; $\mathrm{SR}=$ sustained release; $\mathrm{SSRI}=$ selective serotonin reuptake inhibitor; $\mathrm{TCA}=$ tricyclic antidepressant; $\mathrm{XR}=$ extended release; $(-\mathrm{ve})=$ negative.

and imipramine $[207,224,233-240]$ in panic disorder (Level 1). In meta-analyses, TCAs have demonstrated efficacy for the treatment of panic symptoms and agoraphobia [195-197,226]. Efficacy is generally equivalent to SSRIs, however, since TCAs tend to be less well tolerated and have higher discontinuation rates than SSRIs [195], they are recommended as second-line options.
Other antidepressants: Although there is level 1 evidence to support the use of reboxetine [200,219,244], limited experience with this agent in Canada, and its side effect profile, which includes dry mouth, constipation, and insomnia [244], led to its recommendation as a second-line option. Mirtazapine has demonstrated efficacy for the treatment of panic disorder in several open

Table 15 Recommendations for pharmacotherapy for panic disorder

\begin{tabular}{ll}
\hline First-line & Citalopram, escitalopram, fluoxetine, fluvoxamine, paroxetine, paroxetine CR, sertraline, venlafaxine XR \\
\hline Second-line & Alprazolam, clomipramine, clonazepam, diazepam, imipramine, lorazepam, mirtazapine, reboxetine \\
\hline Third-line & $\begin{array}{l}\text { Bupropion SR, divalproex, duloxetine, gabapentin, levetiracetam, milnacipran, moclobemide, olanzapine, phenelzine, quetiapine, } \\
\text { risperidone, tranylcypromine }\end{array}$ \\
\hline $\begin{array}{l}\text { Adjunctive } \\
\text { therapy }\end{array}$ & $\begin{array}{l}\text { Second-line: alprazolam ODT, clonazepam } \\
\text { Third-line: aripiprazole, divalproex, olanzapine, pindolol, risperidone }\end{array}$ \\
\hline $\begin{array}{l}\text { Not } \\
\text { recommended }\end{array}$ & Buspirone, propranolol, tiagabine, trazodone \\
\hline
\end{tabular}

$\mathrm{CR}=$ controlled release; $\mathrm{ODT}=$ orally disintegrating tablets; $\mathrm{SR}=$ sustained release; $\mathrm{XR}=$ extended release. 
trials [245,246] and one small RCT [203] (Level 2). It appears to be as effective as fluoxetine [203] and may be a useful second-line choice.

Benzodiazepines: Alprazolam [234,249-254], clonazepam [218,250,255-258], lorazepam [251,259,260], and diazepam [261-263] have demonstrated efficacy for the treatment of panic disorder (Level 1). While it has been suggested that alprazolam may be more effective, a metaanalysis found no evidence that it was superior to other benzodiazepines for the treatment of panic disorder [252]. Although benzodiazepines are second-line options, they may be useful at any time during therapy for the short-term management of acute or severe agitation or anxiety. They may also be useful at the initiation of SSRI treatment to hasten response (Level 1) [264-266].

\section{Third-line agents}

MAOIs and RIMAs: Results with moclobemide for the management of panic disorder have been conflicting (Level 1). In clinical trials, moclobemide demonstrated efficacy similar to that of clomipramine and fluoxetine $[204,232]$, but was not superior to placebo [241,242]. However, significant efficacy in more severely ill patients [241], suggests it may be useful in treatment-resistant patients. In a RCT, phenelzine was more effective than placebo and as effective as imipramine (Level 2) [240]. In a small randomized, uncontrolled trial, tranylcypromine demonstrated efficacy for patients with comorbid panic and social anxiety disorders (Level 3) [243].

Atypical antipsychotics: There is some evidence that atypical antipsychotics may have some benefits in the treatment of patients with refractory panic disorder $[217,267,268]$. In a RCT, risperidone monotherapy was as effective as paroxetine (Level 2) [217]. Open-label data also support the use of risperidone [267], olanzapine [268], and quetiapine [267]. There are also open-label data supporting the use of some atypical antipsychotics as adjunctive therapy (see below).

Other therapies: The antidepressants duloxetine [230], milnacipran [231], and bupropion sustained release (SR) $[247,248]$ have shown some efficacy in open trials, as have the anticonvulsants divalproex [272-275] and levetiracetam [276] (all Level 3). In a RCT, gabapentin was superior to placebo in patients who were more severely ill, but not in the overall group (Level 2, negative) [277]. These agents are recommended only as third-line options in patients with refractory panic disorder.

\section{Adjunctive therapy}

There is good evidence that adjunctive clonazepam [264,265] (Level 1), and open-label evidence that adjunctive alprazolam orally-disintegrating tablet (ODT) [266] (Level 3), used short-term ( $<8$ weeks including taper) at the initiation of SSRI treatment, can lead to a more rapid response [264-266].
In a RCT, pindolol added to fluoxetine therapy in patients with treatment-resistant panic disorder was associated with significant improvement in panic disorder symptoms compared with fluoxetine plus placebo (Level 2) [286]. Open-label data also support the use of the atypical antipsychotics aripiprazole [269], olanzapine [270], and risperidone [271] (all Level 3), as well as the anticonvulsant divalproex [281], as adjunctive strategies for patients with treatment-resistant panic disorder.

\section{Not recommended}

Buspirone (Level 1, negative) [254,282], propranolol (Level 2, negative) $[262,284,285]$, tiagabine $[278,279]$ (Level 2, negative), and trazodone (Level 2, negative) [283] have not demonstrated efficacy and are not recommended for the treatment of panic disorder. Carbamazepine (Level 3, negative) [280] also does not appear to be effective in this disorder.

\section{Maintenance pharmacological treatment}

In long-term, open, follow-up studies, citalopram [287,288], fluoxetine [204,288], fluvoxamine [288], paroxetine [288-290], and moclobemide [204], as well as clomipramine [287,289] and imipramine [291,292] demonstrated maintenance of benefits and continued improvements over six months to three years of ongoing treatment. In a RCT, sertraline and imipramine were equally effective over a six month period [224]. However, in another RCT, imipramine was not superior to placebo in the proportion of panic-free patients after eight months of therapy [293].

Venlafaxine XR [294] and imipramine [295] have been shown to prevent relapse in randomized, placebo-controlled, discontinuation studies. After three months of acute treatment, relapse rates were significantly lower with ongoing venlafaxine XR [294] or imipramine [295] therapy compared with switching to placebo during six to 12 months of follow-up.

Benzodiazepines are generally recommended for shortterm use only. However, several trials have demonstrated the benefits of up to two years of alprazolam maintenance therapy $[291,293]$. There was no evidence of tolerance, but up to one-third of patients were unable to discontinue therapy [293]. The efficacy of clonazepam was maintained over a three-year course of treatment [290], and patients who had been asymptomatic for at least one year were able to successfully discontinue the medication, using a slow tapering strategy over four to seven months, and improvement in panic disorder was maintained [296].

\section{Biological and alternative therapies}

Biological therapies: In open-label case series, noninvasive brain stimulation using a radioelectric asymmetric conveyor (REAC) demonstrated efficacy for panic symptoms 
and agoraphobia (Level 3) [297,298]. A small case series suggested repetitive transcranial magnetic stimulation (rTMS) could improve panic and anxiety in patients with panic disorder with comorbid MDD (Level 4) [299]. However, a small RCT found no additional benefit of rTMS compared to sham rTMS as an add-on to SSRI therapy in patients with panic disorder (Level 2, negative) [300].

Alternative therapies: In a RCT, capnometry-assisted respiratory training was as effective as cognitive training in reducing panic symptom severity and panic-related cognitions and improving perceived control (Level 2) [301]. However, breathing training did not significantly improve reactivity or recovery after a respiratory challenge in another small trial (Level 2, negative) [302]. In a RCT, patients with panic disorder randomized to the exercise groups (plus paroxetine or placebo) had a trend toward better improvement compared to relaxation training, but this was not significant (Level 2, negative) [303]. However, in an open cross-over study, acute aerobic exercise was found to reduce anxiety as well as panic attack frequency and intensity in patients with panic disorder compared to a quiet rest condition (Level 3) [304]. These therapies may be useful for some patients; however, more data are needed.

\section{Summary}

As much as $40 \%$ of the general population has experienced a panic attack at some point in their lifetime. However, patients with actual panic disorder experience recurrent, unexpected panic attacks as well as persistent concern or behavioral change around further attacks.

Data support pharmacotherapy, CBT alone, and CBT combined with pharmacotherapy as initial treatments for panic disorder. CBT alone may be insufficient in patients with comorbid moderate-to-severe major depression, or in those with severe, frequent panic attacks, or rapid worsening of agoraphobia, and/or suicidal ideation, as well as in situations where one might consider initial rescue treatment with a benzodiazepine to minimize or stop the panic attacks while waiting the 4-12 weeks for the firstline pharmacotherapy to become effective. Also there are patients who are not motivated to participate in CBT (preferring medication as initial treatment) or are too fearful to engage in any kind of exposure before being treated with a first-line pharmacotherapeutic agent. At the very least, if agoraphobic distress or avoidance persists, these patients need instruction and support to engage in exposure exercises. For panic symptoms, strategies should include exposure; and combined strategies should be considered for patients with agoraphobia. CBT can be effectively delivered in both individual and group settings, as well as via self-help books, virtual reality, and internet-based programs. The benefits of CBT are maintained during follow-up. In addition, data suggest that combination of psychotherapy and pharmacotherapy may be superior to pharmacotherapy alone during follow-up.

Pharmacotherapeutic approaches should begin with a first-line agent. If response to optimal dosing is inadequate or the agent is not tolerated, treatment should be switched to another first-line agent before considering second-line medications. First-line options for the treatment of panic disorder include citalopram, fluoxetine, fluvoxamine, paroxetine, sertraline, venlafaxine XR, escitalopram, or paroxetine CR. Second-line choices include the TCAs (clomipramine and imipramine), mirtazapine, reboxetine, or benzodiazepines (alprazolam, clonazepam, lorazepam, and diazepam).

Patients who do not respond to first- or second-line agents are considered to have treatment-refractory illness. In such patients it is important to reassess the diagnosis and consider comorbid medical (e.g., ischemic heart disease) and psychiatric conditions (e.g., SUDs) that may be affecting response to therapy. Third-line agents, adjunctive therapies, as well as biological and alternative therapies may be useful when patients fail to respond to an optimal treatment trial of first- and second-line therapies used alone and in combination.

\section{Specific phobia Epidemiology}

A specific phobia is an intense fear of a specific object or situation and is usually associated with avoidance of the feared object. The most prevalent phobia types include animal (e.g., insects, snakes), natural environment (e.g., heights, storms, water), situational (e.g., flying, enclosed spaces), and blood-injection-injury (B-I-I) (e.g., blood, dentists, hospitals) [305,306]. Large US and European epidemiologic surveys report lifetime prevalence estimates of $10-13 \%$ and 12 -month prevalence rates of $7-9 \%$ $[2,3,305,307]$. Rates among adolescents may be particularly high with lifetime prevalence estimates of $36.5 \%$ and 12-month prevalence rates of $27.3 \%$ being reported [308]. Specific phobias are more common in women than men [306]. Age of onset is usually in the range of five to 12 years (median: seven years) [2]; however, this varies by type of phobia. Animal and B-I-I phobias generally begin in childhood, whereas situational phobias (e.g., driving phobia, claustrophobia) have a later onset, typically during late adolescence or early adulthood [306].

Specific phobias are associated with significant distress, regardless of the number of feared stimuli reported [305]. Specific phobias have a negative impact on social/occupational functioning and lead to restriction of usual daily activities, which increases with an increasing number of fears [305]. 


\section{Comorbidities}

Specific phobias tend to co-occur with other specific phobias, with less than $10 \%$ of patients having only one fear [305]. The mean number of fears, in one survey, was three [305]. In addition, specific phobias are frequently comorbid with other psychiatric disorders, including SUDs, mood disorders, and other anxiety or related disorders (particularly panic disorder, SAD, and GAD), as well as personality disorders [305,309,310].

\section{Diagnosis}

To receive a DSM-5 diagnosis of specific phobia a patient must experience marked (intense) fear or anxiety about a specific object or situation, which is associated with significant distress or functional impairment (Table 16) [26]. The object or situation will be actively avoided or endured with intense anxiety. Compared to the DSM-IV-TR criteria for specific phobia [144], few changes were made in the DSM-5 [26,306]. Of note, recognition that the fear is excessive or unreasonable has been removed and a new criterion stating "the fear or anxiety is out of proportion to danger posed" has been added. Avoidance has been clarified as "actively avoided" to distinguish the avoidance seen in specific phobias from passive avoidance that may occur for other reasons [26,306].

While the most up-to-date DSM-5 diagnostic criteria are presented here, it is important to note that most of the treatment data described within this section are based on patients meeting DSM-IV criteria (or older).

Specific phobias are delineated into five types: animal type, natural environment type, B-I-I type, situational type, or other type (Table 17) [26]. The fear of contracting an illness has been removed because of high relatedness to OCD and anxiety disorder related to medical condition [26].

Specific phobias can be difficult to distinguish from panic disorder [311]. It is important to consider the focus of apprehension (e.g., fear of crashing while on an airplane versus fear of having a panic attack on an airplane), the types of panic attacks experienced (e.g., expected versus unexpected), and the range of situations associated with fear and avoidance [311].

\section{Table 16 DSM-5 diagnosis of specific phobia}

- Marked fear or anxiety about a specific object or situation (e.g., flying, seeing blood)

- The phobic object or situation almost always provokes immediate fear or anxiety and is actively avoided or endured with marked fear or anxiety

- The fear or anxiety is out of proportion to the actual danger posed by the specific object or situation

- The fear, anxiety, or avoidance is persistent, typically $\geq 6$ months - There is marked distress or functional impairment

Adapted from DSM-5 [26].
Table 17 Specific phobia specifiers in DSM-5

\begin{tabular}{ll}
\hline Specifier & Examples \\
\hline Animal & Spiders, insects, dogs \\
\hline $\begin{array}{l}\text { Natural } \\
\text { environment }\end{array}$ & Heights, storms, water \\
\hline $\begin{array}{l}\text { Blood-injection- } \\
\text { injury }\end{array}$ & Needles, invasive medical procedures \\
\hline Situational & Airplanes, elevators, enclosed spaces \\
\hline Other & $\begin{array}{l}\text { Choking or vomiting. In children, loud sounds or } \\
\text { costumed characters }\end{array}$ \\
\hline
\end{tabular}

Adapted from DSM-5 [26].

\section{Psychological treatment}

Psychosocial interventions, particularly exposure-based treatments, are the treatments of choice and are associated with a high degree of success in providing remission of specific phobias [311]. Both in vivo exposure and virtual reality exposure (VRE) can be effective [57,311,312], with in vivo exposure being shown to be superior to alternative types (e.g., imaginal, virtual reality, etc.) at posttreatment but not at follow-up [57].

In general, exposure-based therapy has been shown to be more effective if: sessions are grouped closely together; exposure is prolonged, real (not imagined), and provided in multiple different settings; and there is some degree of therapist involvement (not entirely self-directed) [32,311]. While one-session treatments have demonstrated efficacy [313], a meta-analysis found that a greater number of sessions predicted more favorable outcomes [57].

There is no evidence that either flooding or gradual exposure is more effective [314], however, progressive exposures are generally more tolerable to patients [311]. An example of graded exposure in a patient with arachnophobia would be to look at pictures of spiders, hold a rubber spider, look at a live spider in a jar, touch the jar containing the spider, stand two feet from a live spider, and finally touch a live spider. This approach can be used to guide exposure depending on the patient's symptom severity and tolerance to each level of exposure.

While a meta-analysis of 33 RCTs of psychological approaches found that treatment outcomes were not moderated by type of specific phobia [57], studies have suggested that certain subtypes may respond more favorably to specific types of treatment (Table 18).

For patients with B-I-I phobias, exposure therapy combined with muscle tension exercises (applied tension) designed to prevent fainting [311] has been shown to be effective $[315,316]$. Use of stress-reducing medical devices, such as decorated butterfly needles and syringes, has been shown to significantly reduce needle phobia and stress in both pediatric and adult patients [317]. CBT reduced avoidance of oral injections and decreased anxiety in patients with dental phobias [318]. 
Table 18 Psychological treatments with demonstrated efficacy in specific phobias

\begin{tabular}{ll}
\hline Psychological treatment & Phobia \\
\hline Exposure-based treatments & All specific phobias [57,311,312] \\
\hline $\begin{array}{l}\text { Virtual reality exposure } \\
\text { Computer-based self-help programs }\end{array}$ & $\begin{array}{l}\text { Heights [327-329], flying [319,321-324], spiders [331,332], claustrophobia } \\
{[330]}\end{array}$ \\
\hline $\begin{array}{l}\text { Applied muscle tension (exposure combined with muscle tension } \\
\text { exercises) }\end{array}$ & Spiders [334,335], flying [323], small animals [336,337] \\
\hline Cognitive therapy and exposure & Blood-injection-injury type [311,315,316] \\
\hline
\end{tabular}

Fear of flying has been effectively treated with group CBT [319,320]. In addition, computer-generated VRE has demonstrated efficacy [319,321-324], which was comparable to standard exposure therapy in several studies [322,324], and can have long-term benefits [325,326]. Bibliotherapy was found to be less effective than VRE or CBT for patients with fear of flying [319]. VRE has also been shown to be effective for patients with a fear of heights [327-329], and those with claustrophobia [330]. This approach may also be useful for treating fears for which in vivo exposure may not be practical (e.g., fear of storms) [32].

Arachnophobia has been successfully treated with in vivo [331] and VR [331,332] exposure, with little difference between the two modalities [331]. A spiderless form of VRE, which presented images that were not spiders, but had some of the characteristics of spiders, was shown to be useful in patients with severe arachnophobia who were reluctant to undergo direct exposure or VRE [333]. An internet-based self-help program was associated with improvement, but was not as effective as one session of in vivo exposure at the post-treatment assessment, although results were similar at follow-up [334]. However, even one session of VRE was associated with greater fear reduction compared to a control group, and may be a useful self-help intervention to reduce fear of spiders [335]. Computer-based self-help has also shown promise for other small-animal phobias (e.g., cockroaches, mice) $[336,337]$.

\section{Combined psychological and pharmacological treatment}

It has been speculated that d-cycloserine, a partial agonist at the NMDA receptor, may improve extinction of fear in patients with phobias undergoing behavioral exposure therapy [338]. In a $\mathrm{RCT}(\mathrm{n}=28), \mathrm{d}$-cycloserine as an adjunct to VRE resulted in significantly larger reductions of acrophobia symptoms compared with VRE alone [338]. In another study $(\mathrm{n}=100)$, adjunctive $\mathrm{d}$-cycloserine did not improve the reduction of spider fears compared to exposure-based therapy alone, however, patients had heightened, but subclinical, spider fears [339].

In two RCTs, use of adjunctive cortisol, a glucocorticoid, significantly enhanced the benefits of exposure therapy compared with placebo in patients with acrophobia $(n=40)$ [340] and arachnophobia $(n=20)$ [341], with evidence suggesting that cortisol may facilitate the extinction of phobic fear at follow-up.

Enhanced emotional memory may be stimulated through elevated noradrenaline levels, and data suggest that yohimbine hydrochloride, a noradrenaline agonist, can facilitate fear extinction. In RCTs, there were no significant VRE-enhancing effects with adjunctive yohimbine compared with placebo in patients with fear of flying $(n=48)$ [342] or claustrophobia $(n=24)$ [343]. However, in the claustrophobia study, patients treated with yohimbine showed greater improvements in outcomes at the one-week follow-up [343].

In contrast, naltrexone was found to render one-session exposure therapy less effective compared with placebo or no treatment in 15 patients with specific phobias (animals) [344].

\section{Long-term effects of psychological treatment}

Long-term treatment of specific phobia is rare. As discussed above, CBT and exposure therapies have demonstrated sustained benefits at long-term follow-up assessments [325,326].

\section{Pharmacological treatment}

There is a minimal role for pharmacotherapy in the treatment of specific phobias, largely due to the lack of research on medications in this condition, and the success of exposure-based therapies [32,311].

Antidepressants have been investigated in two small RCTs $[345,346]$. In a small RCT, paroxetine was significantly more effective than placebo in resolving anxiety in patients with specific phobias $(n=11)$ [345]. Similarly, escitalopram was associated with a strong treatment effect in a small RCT $(n=12)$; however, the trial was under-powered to show statistically significant superiority over placebo on the primary outcome [346]. In addition, cases of successful treatment of flying phobias with fluoxetine [347], and storm phobia with fluvoxamine [348], have been reported.

Benzodiazepines have usually been assessed as adjuncts to exposure therapy, and these studies have found no additional benefit with medication [349-351]. Benzodiazepines are often used in clinical practice to 
provide acute symptom relief when it is necessary for a patient with a specific phobia to face a feared situation (e.g., dental procedure, magnetic resonance imaging [MRI], unexpected flight) [32]. Nasal midazolam has proven useful in facilitating MRI in claustrophobic patients [352,353].

\section{Summary}

Specific phobia is quite common, particularly among adolescents. Patients with specific phobia exhibit an intense fear or anxiety about a specific object or situation which is associated with significant distress or functional impairment. The most prevalent phobia types include animal, natural environment, situational, and B-I-I.

Exposure-based techniques, including virtual exposure, are highly effective, and are the foundation of treatment for specific phobias. Pharmacotherapy is generally unproven, and thus not a recommended treatment for most cases.

\section{Social anxiety disorder Epidemiology}

SAD is one of the most common anxiety disorders, with lifetime prevalence estimates ranging from $8-12 \%$ among the international general population [2,354-356]. It is more common in women than men [355,357-360], and higher rates have been reported in developed (6.1\%) versus developing (2.1\%) countries [361]. SAD has an early age of onset, typically during adolescence (mean 12 years), and tends to have a chronic and unremitting course $[2,362,363]$. Factors such as low educational achievement, low socioeconomic status, being single or separated, and having comorbid MDD have been associated with a higher prevalence of SAD in epidemiological studies [359,360,364].

SAD is associated with significant impairments including problems with educational and occupational performance, family functioning, and an overall reduced QoL $[14,15,17,354,363,365-369]$. SAD also confers a substantial economic burden upon afflicted individuals and society in terms of work days missed and health care costs [370,371]. Canadians with SAD were twice as likely to report at least one disability day in the past two weeks, compared to those without SAD [356].

\section{Psychiatric comorbidity}

SAD is associated with significant comorbidity, with up to $72 \%$ of patients reporting criteria for another psychiatric disorder [372]. The highest rates of comorbidity have been found with MDD and other anxiety or related disorders [355,356,360]. Avoidant personality disorder [373], body dysmorphic disorder [374,375], SUD [356,376], ADHD [377,378], and schizophrenia [379] also commonly occur with SAD.

\section{Diagnosis}

SAD is characterized by a persistent fear that in social and performance situations the individual will say or do something that will lead to humiliation, embarrassment, or negative evaluation by others (Table 19) [26]. Social situations are actively avoided or endured with distress, and the individual recognizes the fears as excessive or unreasonable. The avoidance or anxiety induced by these fears incurs significant functional impairment and distress [144]. Compared to the DSM-IV-TR [144], changes to the diagnostic criteria for SAD in the DSM-5 have been minimal, largely consisting of minor phrasing changes to improve clinical utility [26]. The criterion that the "person recognizes that the fear is excessive or unreasonable" has been changed to "out of proportion to the actual threat posed by the social situation." Since patients with SAD are often unable to recognize that their fear may be excessive the clinician may be in a better position to judge this.

The DSM-IV-TR criteria excluded social fears/avoidance associated with and secondary to medical conditions, however, the DSM-5 recognizes that SAD may be secondary to a medical condition. Some patients experience excessive social anxiety about their medical symptoms (e.g., stuttering, tremulousness from Parkinson's disease, obesity, disfigurement from burns or injury), and may experience disability due to their social anxiety [26].

In addition, the "generalized" subtype specifier included in DSM-IV-TR has been removed, while the "performance only" specifier has been added [26,380] for DSM-5. This change was made because there was little supporting evidence for the generalized specifier, and the evidence that SAD symptoms fall along a continuum of severity characterized by the number of fears [380]. The "performance only" specifier appears to represent a subset of SAD patients typically experiencing

\section{Table 19 DSM-5 diagnosis of SAD (social phobia)}

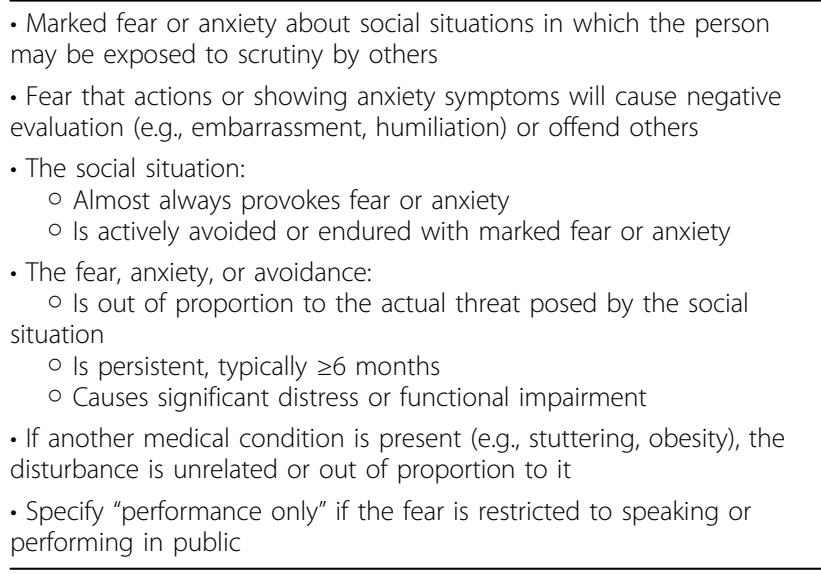

Adapted from DSM-5 [26]. 
impairment from performance fears primarily related to their professional lives [26].

While the most up-to-date DSM-5 diagnostic criteria are presented here, it is important to note that all of the treatment data described within this section are based on patients meeting DSM-IV criteria (or older).

\section{Psychological treatment}

Psychological treatment, in the form of CBT, is considered to be the gold-standard nonpharmacological treatment in SAD. Cognitive techniques involved in CBT for SAD include restructuring and challenging of maladaptive thoughts, while the behavioral component is typically in the form of exposure therapy. The efficacy of CBT compared with placebo, treatment-as-usual, or wait-list conditions, is supported by many RCTs as well as meta-analytic evidence [58,59,70,71,381]. Although results vary, several studies of acute SAD treatment have also found a similar efficacy between CBT and pharmacotherapy [382-387]. Some reports suggest that after treatment discontinuation, gains achieved with CBT may persist longer than those achieved with pharmacotherapy $[388,389]$. CBT for SAD can be administered in group or individual formats. Although some studies have reported that individual CBT is superior to group CBT [390,391], meta-analyses have failed to find significant differences in efficacy between the two modalities [58,59,381].

The treatment literature has also examined the efficacy of the individual components of CBT. There is evidence to support the effectiveness of exposure therapy alone $[389,392]$, however the efficacy of exposure alone compared with CBT is equivocal in the current treatment literature [392-395].

There are several variants of CBT that have been examined in the literature. For example, videotaped feedback was not shown to enhance the effects of exposure-based treatment [396]. However, CBT with VRE was found to be more effective than wait-list control and as effective as CBT with imaginal or in vivo exposure according to two meta-analyses $[80,150]$.

A form of CBT focused on interpersonal behavior found similar improvements in social anxiety compared to standard CBT but also increased relationship satisfaction and social approach behaviors [397]. Evidence to support interpersonal therapy (IPT) in SAD is conflicting [398-400]; while some results have been negative [398], it is likely that IPT is more effective than wait-list control [399], but less effective than traditional CBT [399,400].

Similarly, while less effective than traditional CBT, mindfulness-based therapy (MBT) has been associated with improvements in symptoms of SAD [401]. In addition, small studies of attentional bias training suggest there may be some benefit associated with training patients to disengage from negative social cues, but data are conflicting [402,403].

ICBT is a newer treatment that may increase the availability of CBT for anxiety and mood disorders in the future. Studies have evaluated this treatment in comparison to individual and group CBT. ICBT has demonstrated efficacy in RCTs of SAD, significantly improving social anxiety symptoms compared to wait-list control conditions [404-410]. Most ICBT programs include minimal therapist contact via email [404-410] or telephone $[405,409]$. Many programs involve a component of interaction with other participants through the use of internet discussion groups [411]. However, it remains unclear whether the therapist component is necessary, and studies comparing guided with unguided ICBT have yielded conflicting results. In one RCT, clinician-assisted ICBT was more effective than a self-guided ICBT, and the self-guided ICBT was not significantly better than the wait-list condition [406]. Similarly, a self-help program augmented with minimal therapist contact was more useful than a pure self-help strategy [412]. However, several other RCTs have found that unguided ICBT self-help was as effective as ICBT with therapist involvement $[410,411]$. A few ICBT programs included face-to-face in vivo exposure sessions $[409,413]$, but one RCT found that adding this component did not significantly improve outcomes versus ICBT with self-directed exposure [413]. In addition, several RCTs have shown ICBT (with minimal therapist contact) to be as effective as face-to-face CBT [414,415], while being more cost-effective [416]. As with other RCTs, research on ICBT has involved prescreening of participants in-person or by telephone, with posttreatment and follow-up assessments by telephone or through self-report measures. Little is known about the effectiveness of self-administered treatments (ICBT or self-help books) used with no pre-screening or planned follow-up contacts.

\section{Combined psychological and pharmacological treatments}

When used in combination, pharmacotherapy has not been shown to add to the benefits of CBT in some studies $[387,417]$, while one study found the combination of phenelzine and CBT superior to either modality alone [418]. D-cycloserine has also been found to enhance treatment outcomes when used during exposure exercises as an adjunct to exposure alone $[419,420]$. In addition, a study of psychodynamic group therapy with or without the addition of clonazepam also found combination treatment to be superior to clonazepam treatment alone [421].

\section{Long-term effects of psychological treatment}

The benefits of CBT have been found to be maintained at six to 12 month follow-up visits $[58,382,390,393,409$, $413,422,423]$, with sustained improvement being reported 
at five years posttreatment $[424,425]$. Long-term assessments post-ICBT have shown sustained improvement at one to five years follow-up [409,413,423,424]. Long-term benefits with psychotherapy appear to be more enduring than those of pharmacotherapy after treatment discontinuation $[388,389]$.

\section{Pharmacological treatment}

The management of patients with SAD should follow the principles discussed in Section 2. Pharmacological interventions that have good evidence for efficacy in treating SAD include SSRIs, SNRIs, anticonvulsants, and benzodiazepines. Treatments that have been investigated for use in SAD have been assessed according to the criteria for strength of evidence (Tables 1 and 2) and are summarized in Tables 20 and 21.

\section{First-line agents}

Antidepressants: Meta-analyses demonstrate that SSRIs and SNRIs are significantly more effective than placebo [58,426-429] and RIMAs [426,428] for the treatment of SAD. There is level 1, RCT evidence supporting the use of the SSRIs escitalopram [430,431], fluvoxamine [433-435], fluvoxamine CR [436,437], paroxetine [431,438-444], and sertraline [445-448], as well as the SNRI venlafaxine XR $[439,441,454-456]$, for the first-line treatment of SAD. There is also good evidence for the efficacy of paroxetine CR (Level 2) [452].

Pregabalin: Pregabalin has also demonstrated efficacy versus placebo for the treatment of SAD in RCTs at higher (600 mg/day) but not lower dose levels (150-300 mg/day) (Level 1) [474,475]. Although there is Level 1 evidence for pregabalin, it is not clear how its efficacy compares to that

Table 20 Strength of evidence of pharmacotherapy for SAD

\begin{tabular}{|c|c|c|c|}
\hline Agent & Level of evidence & Agent & Level of evidence \\
\hline \multicolumn{4}{|l|}{ Antidepressants } \\
\hline SSRIs [58,426-429] & 1 & TCAs & \\
\hline Escitalopram [430-432] & 1 & Clomipramine $[458,459]$ & 3 \\
\hline Fluvoxamine [433-435] & 1 & Imipramine [460] & $3(-v e)$ \\
\hline Fluvoxamine CR [436,437] & 1 & MAOIs and RIMAs & \\
\hline Paroxetine [431,438-444] & 1 & Phenelzine $[384,386,418,461,462]$ & 1 \\
\hline Sertraline $[445-448]$ & 1 & Moclobemide $[417,462-466]$ & $1^{*}$ \\
\hline Fluoxetine $[382,387,449]$ & $1^{*}$ & Other antidepressants & \\
\hline Citalopram $[450,451]$ & 2 & Mirtazapine $[467,468]$ & $1^{*}$ \\
\hline Paroxetine CR [452] & 2 & Bupropion SR [469] & 3 \\
\hline Adjunctive paroxetine [453] & 3 & & \\
\hline \multicolumn{4}{|l|}{ SNRIs } \\
\hline Venlafaxine XR $[439,441,454,255,456]$ & 1 & & \\
\hline Duloxetine [457] & 2 & & \\
\hline \multicolumn{4}{|l|}{ Other therapies } \\
\hline Anxiolytics & & Anticonvulsants & \\
\hline Benzodiazepines & & Pregabalin $[474,475]$ & 1 \\
\hline Clonazepam [385,470,471] & 1 & Gabapentin [476,477] & 2 \\
\hline Alprazolam [386] & 2 & Levetiracetam [478-480] & $2(-v e)$ \\
\hline Bromazepam [472] & 2 & Divalproex [481] & 3 \\
\hline \multirow[t]{2}{*}{ Adjunctive clonazepam [473] } & $2(-\mathrm{ve})$ & Tiagabine $[477,482]$ & 3 \\
\hline & & Topiramate [483] & 3 \\
\hline Other treatments & & Atypical antipsychotics & \\
\hline Atenolol $[461,484]$ & 1 (-ve) & Olanzapine [493] & 2 \\
\hline Buspirone $[383,485]$ & 1 (-ve) & Quetiapine $[494,495]$ & $2(-v e)$ \\
\hline Atomoxetine $[486,487]$ & $1^{*}$ & Adjunctive aripiprazole [496] & 3 \\
\hline Propranolol [488] & $2(-v e)$ & Adjunctive risperidone [271] & 3 \\
\hline Selegiline [489] & 3 & & \\
\hline Pergolide [490] & $3(-v e)$ & & \\
\hline Adjunctive buspirone [491] & 3 & & \\
\hline Adjunctive pindolol [492] & $2(-v e)$ & & \\
\hline
\end{tabular}

${ }^{*}$ Conflicting data. $\mathrm{CR}=$ controlled release; $\mathrm{MAOI}=$ monoamine oxidase inhibitor; RIMA = reversible inhibitor of monoamine oxidase $\mathrm{A}$; SNRI = serotoninnorepinephrine reuptake inhibitor; $S R=$ sustained release; $S S R I=$ selective serotonin reuptake inhibitor; $T C A=$ tricyclic antidepressant; $X R=$ extended release; $(-\mathrm{ve})=$ negative . 
Table 21 Recommendations for pharmacotherapy for SAD

\begin{tabular}{ll}
\hline First-line & Escitalopram, fluvoxamine, fluvoxamine CR, paroxetine, paroxetine CR, pregabalin, sertraline, venlafaxine XR \\
\hline Second-line & Alprazolam, bromazepam, citalopram, clonazepam, gabapentin, phenelzine \\
\hline Third-line & $\begin{array}{l}\text { Atomoxetine, bupropion SR, clomipramine, divalproex, duloxetine, fluoxetine, mirtazapine, moclobemide, olanzapine, selegiline, } \\
\text { tiagabine, topiramate }\end{array}$ \\
\hline $\begin{array}{l}\text { Adjunctive } \\
\text { therapy }\end{array}$ & $\begin{array}{l}\text { Third-line: aripiprazole, buspirone, paroxetine, risperidone } \\
\text { Not recommended: clonazepam, pindolol }\end{array}$ \\
\hline $\begin{array}{l}\text { Not } \\
\text { recommended }\end{array}$ & Atenolol, buspirone, imipramine, levetiracetam, propranolol", quetiapine \\
\hline
\end{tabular}

$\mathrm{CR}=$ controlled release; $\mathrm{SR}=$ sustained release; $\mathrm{XR}=$ extended release.

*Beta-blockers have been successfully used in clinical practice for performance situations such as public speaking.

Note: although there is limited evidence for citalopram in SAD, it is likely as effective as the other SSRIs, in contrast there are negative trials of fluoxetine in SAD suggesting it may be less effective than other SSRIs [382,449].

of SSRIs. In addition, SSRIs may have a broader spectrum of efficacy for common comorbid conditions.

\section{Second-line agents}

Benzodiazepines: In RCTs, the benzodiazepines clonazepam (Level 1) [470][385,471], alprazolam [386], and bromazepam [472] (both Level 2) have demonstrated efficacy in the treatment of SAD.

Although, a meta-analysis found benzodiazepines to be as effective as SSRIs [58], these agents are recommended as second-line options because of the lack of effect on common comorbidities and the potential for abuse/ dependence in individuals with a history of SUDs.

Antidepressants: In RCTs, citalopram was found to be significantly more effective than placebo [451], and as effective as moclobemide [450] (Level 2). Although there is limited evidence for citalopram in SAD, it is likely as effective as the other SSRIs.

The efficacy of phenelzine has been established in multiple RCTs (Level 1) [384,386,418,461,462]; however, this agent is recommended as a second-line option because of concerns regarding dietary restrictions, drug interactions, and the potential for hypertensive crisis.

Anticonvulsants: Gabapentin was significantly more effective than placebo in a RCT [476], and as effective as tiagabine in a small cross-over study (Level 2) [477].

\section{Third-line agents}

Antidepressants: Results with fluoxetine have been mixed (Level 1, conflicting) [382,387,449]. A large RCT found that fluoxetine was more effective than placebo and as effective as CBT [387]. However, in two other small RCTs, fluoxetine alone or when added to selfexposure showed no benefit over placebo, with or without self-exposure [382,449]. These negative trials with fluoxetine suggest it may be less effective than other SSRIs $[382,449]$.

Similarly, results with moclobemide have also been mixed (Level 1, conflicting) [417,462-466], with some RCTs demonstrating significantly higher response rates with moclobemide compared with placebo (Level 1) [462-464], while others have not $[465,466]$. Moclobemide was found to be superior to CBT early in treatment; however, after six months CBT was found to be superior.

Data from two small RCTs assessing mirtazapine were also mixed (Level 1, conflicting), with one showing significant improvements over placebo [468] and the other showing no differences [467].

In a dose-finding study in which patients treated with open-label duloxetine $60 \mathrm{mg} /$ day were randomized to continue or double their dose, both doses improved symptoms, but there was no significant advantage to the higher dose (Level 2) [457].

Small open-label trials have also suggested that bupropion SR [469] and clomipramine [458,459] (both Level 3) may be effective in patients with SAD.

Anticonvulsants: Open-label studies have demonstrated some efficacy with divalproex [481], topiramate [483], and tiagabine [482] (all Level 3). In addition, tiagabine was comparable to gabapentin in a small RCT, crossover study in eight adults [477].

Other treatments: Olanzapine was effective in a small RCT (Level 2) [493], and selegiline demonstrated efficacy in a small, open-label trial (Level 3) [489]. In a RCT, atomoxetine significantly improved SAD symptoms compared with placebo [487]; however, in a another small RCT, atomoxetine showed no significant difference in outcomes compared with placebo (Level 1, conflicting) [486].

All of these agents are recommended as third-line options, and may be useful in refractory patients after first- and second-line monotherapies and adjuncts have been unsuccessful.

\section{Adjunctive therapy}

Adjunctive strategies have generally been studied in patients who have had an inadequate response to antidepressant therapy and can be considered for patients with treatment-resistant SAD.

Third-line adjunctive therapies: Open-label studies and case series have suggested that patients with refractory 
SAD may benefit from adjunctive therapy with aripiprazole [496], risperidone [271], buspirone [491], or paroxetine [453] (all Level 3).

Not recommended adjunctive or combination therapies: In RCTs, clonazepam [473] combined with paroxetine and pindolol augmentation of paroxetine [492] (both Level 2, negative) were not significantly superior to placebo in augmenting the effects of SSRI treatment for SAD.

\section{Not recommended}

In RCTs there was no evidence of benefits with the betablockers atenolol (Level 1, negative) $[461,484]$ or propranolol (Level 2, negative) [488], or for the following treatments: buspirone [383,485], levetiracetam [478-480] (both Level 1, negative), or quetiapine (Level 2, negative) [494,495]. These agents are not recommended for SAD. Imipramine [460] and pergolide (both Level 3, negative) [490] also do not appear to be effective in this disorder.

\section{Maintenance pharmacological treatment}

Long-term therapy has been evaluated in relapse prevention and naturalistic follow-up studies. Relapse-prevention studies are those in which responders to medication are randomized to continued active treatment or placebo. A meta-analysis of four relapse prevention studies included 760 patients with SAD and found a highly significant reduction in relapse rates with continued SSRI treatment compared with placebo over three to six months. The relative risk (RR) for relapse was 0.39 (95\% CI 0.300.49 ) and number needed to treat (NNT) was 3.57 (95\% CI 2.94-4.76) [497]. The anticonvulsant pregabalin has also demonstrated reductions in relapse rates over six months [498].

In RCTs, escitalopram [431], fluvoxamine CR [499], and venlafaxine XR [456] have demonstrated continued improvement compared with placebo over approximately six months. Additional open follow-up data support the long-term efficacy of moclobemide over six to 24 months $[464,500]$.

\section{Biological and alternative therapies}

Biological therapies: In an open-label study, neuro psycho physical optimization-radio electric asymmetric conveyor (NPPO-REAC) (a brain stimulation technique) was as effective as sertraline for the treatment of SAD (Level 3) [501].

Alternative therapies: St John's wort failed to demonstrate superiority over placebo, and is not recommended for the treatment of SAD (Level 2, negative) [502].

\section{Summary}

SAD is one of the most common anxiety disorders, occurring more often in women than men. SAD has a negative impact on QoL, functional and occupational outcomes, and is often associated with other comorbid disorders, including MDD and other anxiety and related disorders. SAD is characterized by intense fear or anxiety relating to social or performance situations where the individual is exposed to scrutiny by others. These situations are often actively avoided.

CBT and exposure therapy alone are effective first-line options for the treatment of SAD, although limited data suggest that CBT may be more effective in maintaining benefits during follow-up. VRE and internet-based programs have also demonstrated efficacy. The benefits of CBT are maintained over one to five years of follow-up. CBT and pharmacotherapy appear to have similar efficacy for the acute treatment of SAD, but after treatment discontinuation, gains achieved with CBT appear to persist longer than those achieved with pharmacotherapy. In most studies, adding pharmacotherapy has not been shown to increase the benefits of CBT.

Pharmacotherapeutic approaches should begin with a first-line antidepressant such as escitalopram, fluvoxamine, fluvoxamine $C R$, paroxetine, paroxetine $C R$, sertraline, or venlafaxine $\mathrm{XR}$, or the anticonvulsant pregabalin. If response to optimal doses is inadequate or the agent is not tolerated, therapy should be switched to another first-line agent before considering a second-line medication. Second-line choices include the benzodiazepines alprazolam, bromazepam, and clonazepam, as well as citalopram, gabapentin, and phenelzine. Pregabalin has also been shown to maintain benefits and prevent relapse in a six-month study.

Patients who do not respond to several medication trials and/or CBT are considered to have treatmentrefractory illness. In such patients it is important to reassess the diagnosis and consider comorbid medical and psychiatric conditions that may be affecting response to therapy. Third-line agents and adjunctive therapies may be useful when patients fail to respond to optimal treatment trials of first- and second-line therapies used alone and in combination.

\section{Generalized anxiety disorder Epidemiology}

The estimated 12-month prevalence of GAD ranges from $1-4 \%$, and the lifetime prevalence is approximately $6 \%$ $[2,3,16,503]$. GAD is more frequent in Caucasians compared to other groups [504]. The usual age of onset varies and may be bimodal with the median age of onset being approximately 31 years [2] and mean age of onset being 32.7 years [505]. The prevalence of GAD is estimated to be $3 \%$ in children and $10.8 \%$ in adolescents [506], with the age of onset for children and adolescents being between ages 10 and 14 [507]. Some data suggest that women may be two to three times more likely to suffer from GAD than men $[16,508]$, and GAD may be more 
common in older adults $[509,510]$. This disorder is reportedly frequently under-recognized with less than one-third of patients being adequately treated [511,512]. This is further complicated in children because of the previous designation of Overanxious Disorder of Childhood and its possible differentiation of childhood GAD from GAD in adults.

GAD is associated with functional $[15,511,513]$, occupational [511], and QoL impairments [16,511], as well as substantial economic costs [511,514]. In addition, in primary care $60-94 \%$ of patients with GAD report painful physical symptoms $[515,516]$, and these were the main reason for initial presentation to a physician in $72 \%$ of cases [516].

\section{Comorbidity}

GAD is associated with high rates of comorbid psychiatric conditions including other anxiety or related disorders and MDD [16]. The risk of medical conditions is also elevated [16], including pain syndromes [16,517], hypertension [16], as well as cardiovascular and gastric conditions $[16,518]$. The presence of comorbid depression increases the severity of illness, functional impairment [519], and economic costs [514].

\section{Diagnosis}

GAD is characterized by excessive anxiety and worry about multiple events or activities such as school or work difficulties, which is apparent on a majority of days over the previous six months (Table 22) [26]. In addition, GAD is associated with restlessness, muscle tension, fatigue, concentration difficulties, irritability, and sleep issues [26].

The diagnostic criteria for GAD underwent one minor revision in the DSM-5 [26] compared to the DSM-IVTR [144], the requirement that the disturbance not occur exclusively during a mood, psychotic, or pervasive developmental disorder was removed. However, it remains important to note that most of the treatment data described within this section are based on patients meeting DSM-IV criteria (or older).

\section{Psychological treatment}

Meta-analyses clearly demonstrate that CBT significantly reduces GAD symptoms and is markedly more effective

\section{Table 22 DSM-5 diagnosis of GAD}

- Excessive anxiety and worry (apprehensive expectation) about a number of events or activities (e.g., school/work performance)

- The individual finds it difficult to control the worry

- Excessive anxiety and worry are associated with $\geq 3$ of the following symptoms (with at least some occurring more days than not for $\geq 6$ months):

o Restlessness or feeling keyed-up or on edge, being easily fatigued, difficulty concentrating, irritability, muscle tension, or sleep disturbance - The disturbance causes clinically significant distress or functional impairment

Adapted from DSM-5 [26]. than placebo or wait-list control conditions for GAD (Level 1) $[55,64,65,70,520]$. Few studies have compared CBT and pharmacotherapy alone in the same trial, but the magnitude of benefits appear to be comparable for both groups [521-523]. Individual and group therapy appear to be equally effective in terms of anxiety symptom reduction, but individual therapy may lead to earlier improvement in worry and depression symptoms [65,520].

The intensity of therapy was assessed in a meta-analysis of 25 studies [65]. Regimens including fewer than eight sessions were as effective as those of eight or more for anxiety symptoms, but the more intense regimens were more effective in improving symptoms of worry and depression compared with fewer sessions [65].

Several studies have demonstrated the utility of internetbased or computer-based CBT programs [79,524-526]. ICBT has been shown to be significantly more effective than wait-list control $[79,524,525]$, with benefits being maintained at long-term follow-up [525]. In addition, a peer-to-peer cognitive self-therapy program was as effective as treatment-as-usual, with a decreased need for therapist contact [527].

A meta-analysis of five trials found no significant differences between CBT and relaxation therapy [55]. However, more recent studies suggest that applied relaxation has limited efficacy [528-530]. One RCT found little evidence that patients with GAD can learn to relax in therapy or that a decrease in activation is associated with a reduction in anxiety [529]. Balneotherapy, a relaxation therapy involving spa-related treatments, demonstrated potential advantages over SSRI pharmacotherapy in improving anxiety scores and response rates in patients with GAD in a large RCT [531]; however, while this study may be interesting, concerns pertaining to blinding and potential bias indicate further study is needed [531].

Several research-based variables have been specifically identified among individuals with GAD in order to generate evidence-based $\mathrm{CBT}$ protocols for GAD, including: intolerance of uncertainty, poor problem-solving confidence, as well as positive and negative metacognitive beliefs about the function or utility of worry [532]. Specific psychotherapeutic protocols based upon models of the disorder that target variables underlying GAD have been developed to individualize therapy. Acceptance-based behavior therapy [533], meta-cognitive therapy [528,534], CBT targeting intolerance of uncertainty [530], and adjunctive MBCT [184] have demonstrated efficacy for the treatment of GAD. Targeting worry and relaxation [535], as well as looming vulnerability (the tendency to generate and maintain internal scenarios of increasing risk and danger) [536], may also be beneficial.

Psychodynamic therapy may also be of benefit, however the research findings to date are unclear. A RCT 
found that short-term psychodynamic psychotherapy was as effective as CBT in improving anxiety scores, but CBT was superior on measures of worry and depression [537]. Another study found no significant differences between brief psychodynamic therapy, pharmacotherapy, or the combination [523].

No significant benefits were found with the addition of interpersonal and emotional processing therapy to CBT when compared with CBT plus supportive listening [538]. However, pretreatment motivational interviewing as an adjunct to CBT was shown to help reduce resistance to therapy, improve homework compliance, and improve worry outcomes - this strategy may be particularly useful in more severe cases [539,540].

In clinical practice, the approach may need to be individualized to the problems experienced by the patient.

\section{Psychological and pharmacological treatment}

Few data are available on the use of combined psychological and pharmacological treatment. A meta-analysis concluded that combination pharmacotherapy and CBT was more effective than CBT alone at posttreatment but not at six-month follow-up [83]. While large effect sizes were found for GAD, data were available from only two studies, and these compared CBT plus diazepam or buspirone with CBT alone [83]. Compared to pharmacotherapy alone, the few studies that have assessed the benefits of adjunctive psychotherapy have been conflicting $[184,523,541,542]$. One study suggested benefits of the combination [184], while two other studies did not $[523,541]$. However, adjunctive CBT was shown to facilitate benzodiazepine tapering in patients with GAD [542].

There is no current evidence to support the routine combination of CBT and pharmacotherapy. However, as in other anxiety and related disorders, when patients do not benefit from CBT or have a limited response, a trial of pharmacotherapy is advisable. Similarly, patients who show limited benefit from pharmacotherapy may benefit from CBT.

\section{Long-term effects of psychological treatment}

Long-term follow-up data from a meta-analysis [520] and RCTs [523,525,535,543] suggest that benefits of psychological treatments are maintained at one to three years follow-up after treatment.

\section{Pharmacological treatment}

The management of patients with GAD should follow the principles discussed in Section 2. Pharmacological interventions that have good evidence for efficacy in treating GAD include SSRIs, SNRIs, TCAs, benzodiazepines, pregabalin, quetiapine $\mathrm{XR}$, and other therapies. Treatments that have been investigated for use in GAD have been assessed according to the criteria for strength of evidence (Tables 1 and 2 ) and are summarized in Tables 23 and 24 .

\section{First-line agents}

Antidepressants (SSRIs \& SNRIs): Evidence from RCTs supports the use of SSRIs including escitalopram [544-552] and sertraline [556,559-561], as well as the SNRIs duloxetine [566-571] and venlafaxine XR [548,553,570-580] (all Level 1) for the first-line treatment of GAD. Similar evidence exists for paroxetine [546,547,553-558] supporting its use as a first-line option. Paroxetine CR has a similar active ingredient, and although there are less data supporting its use, it is likely interchangeable with paroxetine as a first-line agent (Level 3) $[564,565]$. In head-to-head comparisons, the efficacy of SSRIs and SNRIs appear to be similar $[546,547,549,556,558,570,571]$. Some data suggest that escitalopram may be less effective than venlafaxine XR [548] or quetiapine XR [551]. Efficacy of venlafaxine was similar to pregabalin in one RCT [576], but less effective in another [577].

Other antidepressants: In two 12-week, double-blind RCTs, agomelatine was found to be more effective than placebo (Level 1) [584,585], and as effective as escitalopram [585].

Pregabalin: The anticonvulsant pregabalin was more effective than placebo in RCTs [576,577,592,593,597,613] and as effective as benzodiazepines [592,593,597] in patients with GAD (Level 1). Pregabalin was more effective than venlafaxine XR in one RCT [577], but equivalent in another [576].

\section{Second-line agents}

Benzodiazepines: Alprazolam [589-593], bromazepam $[589,594]$, diazepam $[583,589,595,596]$, and lorazepam [589,593,597-601] all have demonstrated efficacy for the treatment of GAD (all Level 1). While these agents have level 1 evidence for efficacy, they are recommended as second-line therapy, and usually only for short-term use, because of side effects, dependence, and withdrawal issues.

TCAs and other antidepressants: In RCTs, imipramine was superior to placebo and as effective as benzodiazepines for the treatment of GAD (Level 1) [553,581-583]. However, because of side effects and potential toxicity in overdose, imipramine is recommended as a secondline option. While there are little data on bupropion XL (Level 2), in a 12-week RCT in patients with GAD it was as effective as escitalopram (a first-line option), supporting its use as a second-line option [549].

Vortioxetine is a so-called "serotonin modulator" because of its activity in a variety of serotonin receptors. Results from two similar, eight-week, placebo-controlled RCTs with vortioxetine were conflicting, with one trial being positive [587] and the other negative (Level 1, conflicting) [586]. The differences in outcomes may be related to differences in recruitment between the two studies [623], and data suggest that vortioxetine may be useful in GAD. 
Table 23 Strength of evidence for pharmacotherapy for GAD

\begin{tabular}{|c|c|c|c|}
\hline Agent & Level of evidence & Agent & Level of evidence \\
\hline \multicolumn{4}{|l|}{ Antidepressants } \\
\hline SSRIs & & TCAs & \\
\hline Escitalopram [544-552] & 1 & Imipramine [553,581-583] & 1 \\
\hline Paroxetine $[546,547,553-558]$ & 1 & Other antidepressants & \\
\hline Sertraline $[556,559-561]$ & 1 & Agomelatine $[584,585]$ & 1 \\
\hline Citalopram [562] & 3 & Vortioxetine [586,587] & $1^{*}$ \\
\hline Fluoxetine [563] & 3 & Bupropion XL [549] & 2 \\
\hline Paroxetine CR $[564,565]$ & 3 & Trazodone [583] & 2 \\
\hline SNRIs & & Mirtazapine [588] & 3 \\
\hline Duloxetine [566-571] & 1 & & \\
\hline Venlafaxine XR [548,553,570-580] & 1 & & \\
\hline \multicolumn{4}{|l|}{ Other therapies } \\
\hline Anxiolytics & & Atypical antipsychotics & \\
\hline Benzodiazepines & & Quetiapine XR [551,557,602,603] & 1 \\
\hline Alprazolam [589-593] & 1 & Adjunctive quetiapine $[565,604,605]$ & $1^{*}$ \\
\hline Bromazepam [589,594] & 1 & Adjunctive risperidone $[606,607]$ & $1^{*}$ \\
\hline Diazepam [583,589,595,596] & 1 & Adjunctive olanzapine [608] & 2 \\
\hline \multirow[t]{3}{*}{ Lorazepam [589,593,597-601] } & 1 & Adjunctive aripiprazole $[269,609]$ & 3 \\
\hline & & Adjunctive quetiapine XR [610] & 3 \\
\hline & & Adjunctive or monotx ziprasidone $[611,612]$ & $2(-v e)$ \\
\hline Anticonvulsants & & Other treatments & \\
\hline Pregabalin $[576,577,592,593,597,613]$ & 1 & Buspirone $[108,561,572,589,598,618,619]$ & 1 \\
\hline Divalproex chrono [614] & 2 & Hydroxyzine $[594,619,620]$ & 1 \\
\hline Tiagabine $[615,616]$ & $1(-\mathrm{ve})$ & Pexacerfont [552] & $2(-v e)$ \\
\hline \multirow[t]{2}{*}{ Adjunctive pregabalin [617] } & 2 & Propranolol [621] & $2(-v e)$ \\
\hline & & Memantine [622] & $4(-\mathrm{ve})$ \\
\hline
\end{tabular}

${ }^{*}$ Conflicting data. SNRI = serotonin-norepinephrine reuptake inhibitor; SSRI = selective serotonin reuptake inhibitor; TCA = tricyclic antidepressant; $\mathrm{XL}=$ extended release; $X R=$ extended release; $(-v e)=$ negative.

Quetiapine XR: There is good evidence for the efficacy of quetiapine XR for the management of GAD (Level 1) $[551,557,602,603]$. Two meta-analyses [115,116] concluded that quetiapine was significantly superior to placebo and equivalent to antidepressants [115] for the treatment of GAD. However, quetiapine was associated with more weight gain and sedation, and higher dropout rates due to adverse events compared with placebo or antidepressants $[115,116]$. Due to tolerability and long-term safety concerns with atypical antipsychotics, this treatment is recommended as a second-line option for patients who cannot be provided antidepressants or benzodiazepines.

Other treatments: Buspirone was more effective than placebo and as effective as benzodiazepines in several

Table 24 Recommendations for pharmacotherapy for GAD

\begin{tabular}{ll}
\hline First-line & Agomelatine, duloxetine, escitalopram, paroxetine, paroxetine $C R$, pregabalin, sertraline, venlafaxine $X R$ \\
\hline Second-line & $\begin{array}{l}\text { Alprazolam*, bromazepam*, bupropion } X L^{*} \text {, buspirone, diazepam*, hydroxyzine, imipramine, lorazepam*, quetiapine } X R^{*}, \\
\text { vortioxetine }\end{array}$ \\
\hline Third-line & Citalopram, divalproex chrono, fluoxetine, mirtazapine, trazodone \\
\hline $\begin{array}{l}\text { Adjunctive } \\
\text { therapy }\end{array}$ & $\begin{array}{l}\text { Second-line: pregabalin } \\
\text { Third-line: aripiprazole, olanzapine, quetiapine, quetiapine XR, risperidone } \\
\text { Not recommended: ziprasidone }\end{array}$ \\
\hline $\begin{array}{l}\text { Not } \\
\text { recommended }\end{array}$ & Beta blockers (propranolol), pexacerfont, tiagabine
\end{tabular}

$\mathrm{CR}=$ controlled release; $\mathrm{XL}=$ extended release; $\mathrm{XR}=$ extended release.

*Note: These have distinct mechanisms, efficacy and safety profiles. Within these second-line agents, benzodiazepines would be considered first in most cases, except where there is a risk of substance abuse, while bupropion XL would likely be reserved for later. Quetiapine XR remains a good choice in terms of efficacy, but given the metabolic concerns associated with atypical antipsychotic, it should be reserved for patients who cannot be provided antidepressants or benzodiazepines. Please refer to text for further rationale for the recommendations. 
RCTs (Level 1) [108,561,572,589,598,618,619]. There are limited data comparing buspirone to antidepressants, with it being less effective than venlafaxine XR in one study [572], but as effective as sertraline in another [561]. Limited effectiveness in clinical practice relegates buspirone to a second-line agent.

Hydroxyzine has demonstrated efficacy superior to placebo and similar to benzodiazepines and buspirone in RCTs (Level 1) [594,619,620]; however, clinical experience with this agent in the treatment of GAD remains limited.

\section{Third-line agents}

The following agents are recommended as third-line options because of limited data, side effects, or lack of clinical experience as a primary therapy for the treatment of GAD.

Antidepressants: In open-label studies or case series, the antidepressants citalopram [562], fluoxetine [563], paroxetine CR [564,565], and mirtazapine [588] have demonstrated efficacy in patients with GAD (all Level 3). In a $\mathrm{RCT}$, trazodone was as effective as diazepam (Level 2) [583].

Other treatments: Divalproex chrono was superior to placebo for the treatment of GAD (Level 2) [614], however this formulation is not widely available.

\section{Adjunctive therapy}

Adjunctive strategies have generally been studied in patients who have had an inadequate response to SSRI therapy, and can be considered for patients with treatment-resistant GAD.

Second-line adjunctive therapies: Adjunctive pregabalin demonstrated good efficacy in a large RCT in patients with GAD who had an inadequate response to prior treatments (Level 2) [617].

Third-line adjunctive therapies: A meta-analysis of five RCTs of adjunctive atypical antipsychotics found no significant improvement in response rates but higher discontinuation rates versus placebo in patients with refractory GAD [116].

Two RCTs suggest that adjunctive risperidone (Level 1, conflicting) $[606,607]$ may be useful in some patients, but in the larger RCT it demonstrated superiority over placebo only in patients with moderate to severe residual symptoms at baseline [607]. Similarly, data on adjunctive quetiapine have been inconsistent (Level 1, conflicting) [565,604,605], with one RCT being negative [565], while another, unblinded RCT showed some, but limited benefits [605]. Adjunctive olanzapine demonstrated efficacy in a small RCT in patients who remained symptomatic after six weeks of SSRI therapy [608]. Adjunctive treatment with quetiapine XR [610] or aripiprazole [269,609] (both Level 3) also had some benefit in open trials.

Because of the limited evidence for efficacy and their potential for weight gain and metabolic side effects, atypical antipsychotics should be reserved for highly treatment-refractory cases of GAD, and other than quetiapine XR, used only as an adjunctive treatment.

Not recommended adjunctive therapies: Ziprasidone does not appear to be effective as adjunctive therapy (Level 2, negative) [611].

\section{Not recommended}

Propranolol [621] and pexacerfont [552] (both Level 2, negative) have not demonstrated efficacy and are not recommended in the treatment of GAD. While a small randomized, open-label trial suggested that tiagabine was as effective as paroxetine, the results of three placebocontrolled RCTs do not support the efficacy of tiagabine in patients with GAD (Level 1, negative) [615,616]. Memantine also does not appear to be effective in this disorder (Level 4, negative) [622].

\section{Maintenance pharmacological treatment}

Long-term therapy has been evaluated in relapse prevention and naturalistic follow-up studies. Relapse-prevention studies are those in which responders to SSRI therapy are randomized to continued active treatment or placebo. A meta-analysis of three relapse prevention studies included 1342 patients with GAD and found a highly significant reduction in relapse rates with continued SSRI treatment compared with placebo over six to 12 months (odds ratio for relapse was 0.20) [497].

In RCT discontinuation studies, duloxetine [624], escitalopram [625], paroxetine [626], and venlafaxine XR [627] have demonstrated significantly lower relapse rates over six to 18 months in the range of $10-20 \%$ with active treatment compared to $40-56 \%$ with placebo. Pregabalin [628] and quetiapine XR [629] have also demonstrated significantly lower relapse rates over six to 12 months of continued treatment in discontinuation trials.

In long-term RCT studies, escitalopram [546], paroxetine [546], and venlafaxine XR [578,579] have demonstrated continued improvement compared with placebo over approximately six months.

\section{Biological and alternative therapies}

In general, these therapies may be useful for some patients; however, more data are needed.

Biological therapies: In a small open trial, rTMS was effective as monotherapy or as an adjunct to SSRIs in patients with GAD (Level 3) [630], and improvements were largely maintained six months after treatment [631].

Alternative therapies: Several herbal preparations have demonstrated efficacy comparable to lorazepam for the treatment of GAD including silexan (lavender oil) (Level 1) [600,632] and Galphimia glauca extract (Level 2) [601]. Cochrane meta-analyses found two studies of passiflora (passion flower) indicating it was as effective as benzodiazepines (Level 2) [633], and one study of valerian which found no significant differences between placebo, valerian, or diazepam (Level 2, negative) [634,635]. 
Unfortunately, because these preparations are poorly standardized and have substantial variation in proportion of the active ingredient in different products, they cannot be widely recommended.

A RCT of adjunctive resistance training (weightlifting) or aerobic exercise found significant symptomatic improvements compared to a wait-list condition (Level 2) [636]. A systematic review included four studies of acupuncture in GAD or anxiety neurosis, and while all trials reported positive findings, methodological details were lacking and the authors concluded that there was insufficient evidence to determine efficacy (Level 2) [637]. Open-label studies suggest that adjunctive meditation and yoga-based treatments may be useful in patients with GAD (Level 3) [638,639].

Not recommended alternative therapy: In a $\mathrm{RCT}$, there were no significant improvements with bright light therapy compared with placebo (Level 2, negative) [640], and this treatment is not recommended.

\section{Summary}

The lifetime prevalence of GAD is approximately $6 \%$, it is more frequent in women than in men, with age of onset reflecting a bimodal distribution (onset in lateteens to early-twenties, and again in the 30s and 40s). GAD is associated with substantial functional impairment and a high prevalence of comorbid psychiatric and medical disorders. According to DSM-5 criteria, GAD is characterized by excessive anxiety and worry about multiple situations and is associated with restlessness, muscle tension, and behavioral changes.

CBT is an effective first-line option for the treatment of GAD and is as effective as pharmacotherapy. Internetbased and computer-based CBT have also demonstrated efficacy. Evidence does not support the routine combination of CBT and pharmacotherapy, but when patients do not benefit from CBT, a trial of pharmacotherapy is advisable, and vice versa.

Pharmacotherapeutic approaches should begin with one of the first-line options including an SSRI such as escitalopram, paroxetine, or sertraline, an SNRI such as duloxetine or venlafaxine XR, or other antidepressant such as agomelatine. The anticonvulsant pregabalin is also a recommended first-line therapy.

If response to optimal doses is inadequate or the agent is not tolerated, therapy should be switched to another firstline agent before considering second-line medications. Second-line choices include bupropion XL, buspirone, hydroxyzine, imipramine, quetiapine $\mathrm{XR}$, vortioxetine, as well as the benzodiazepines, alprazolam, bromazepam, diazepam, and lorazepam.

Patients who do not respond to multiple courses of therapy are considered to have treatment-refractory illness. In such patients it is important to reassess the diagnosis and consider comorbid medical and psychiatric conditions that may be affecting response to therapy. Third-line agents, adjunctive therapies, as well as biological and alternative therapies may be useful when patients fail to respond to an optimal treatment trial of first- and second-line therapies used alone and in combination.

\section{Obsessive-compulsive disorder Epidemiology}

OCD is a relatively uncommon, yet severe, mental disorder, with an estimated lifetime and 12-month prevalence of $1.0-2.3 \%$ and $0.7 \%-1.2 \%$ in adults, respectively $[2,3,641,642]$. Mean age of onset of OCD is $\sim 20$ years of age, but symptoms can occur below the age of 10, with few new cases after the early 30s [2,641,643]. Rates of treatment-seeking have been estimated to be only about $14-56 \%$ of patients, suggesting that OCD may be underrecognized and under-treated $[644,645]$. Social isolation, history of physical abuse, and negative emotionality are risk factors for the development of OCD [646].

OCD is associated with a substantial negative impact on QoL for both patients $[647,648]$ and their caregivers [649]. Patients experience cognitive, social, and occupational impairments $[642,645,650,651]$. In addition, up to one-quarter of patients with OCD have attempted suicide $[645,652]$. OCD symptoms are associated with increased rates of health care utilization compared to those without OCD symptoms [642], with health care costs estimated at $\$ 10.6$ billion/year (2005) in the US [653].

\section{Comorbidity}

About $60-90 \%$ of patients with OCD also have a comorbid disorder $[641,645]$. Patients with OCD or OCD symptoms have a three-times higher rate of comorbidity compared to those without OCD symptoms [642]. Common comorbidities include mood, anxiety, and somatoform disorders, as well as SUDs, psychotic disorders, and bipolar disorders $[641,642,645]$.

\section{Diagnosis}

A diagnosis of OCD requires the presence of obsessions and/or compulsions (Table 25) [26]. Obsessions are defined as recurrent, persistent, and intrusive thoughts, images, or urges that cause marked anxiety, and compulsions are defined as repetitive behaviors or mental acts that the patient feels compelled to perform to reduce the obsession-related anxiety [26]. The obsessions or compulsions are time consuming and cause significant impairment in social or occupational functioning.

In the DSM-5, OCD has been moved from the "anxiety disorders" [144] to a new diagnostic category called "obsessive-compulsive and related disorders." In addition to OCD, this new category also includes diagnostic criteria for body dysmorphic disorder, hoarding disorder, 


\section{Table 25 DSM-5 diagnosis of OCD}

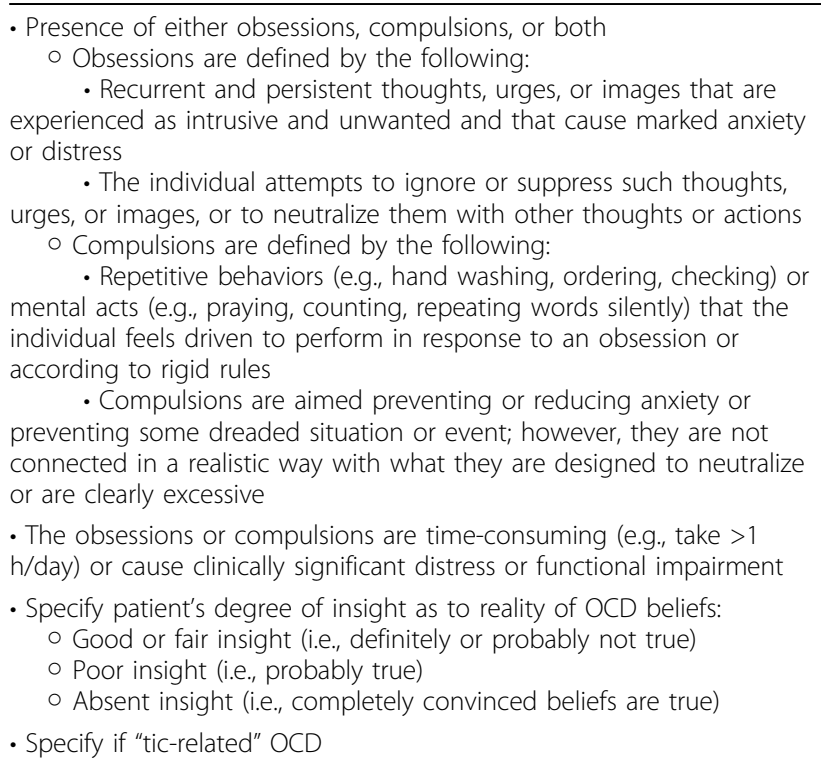

Adapted from DSM-5 [26].

hair-pulling disorder (trichotillomania), and skin picking disorder [26].

Most of the other modifications to the OCD diagnostic criteria in the DSM-5 were minor wording changes designed to enhance clarity or further operationalize concepts that were considered too vague [26]. In particular, the definitions of obsessions and compulsions were clarified and simplified $[26,654]$. The requirement that the patient recognizes that the obsessions or compulsions are "excessive or unreasonable" has been deleted, since these terms are subject to interpretation and patients can have varying levels of insight. As a result, the previous DSMIV-TR specifier of "poor insight" has been expanded to include: good or fair, poor, and absent insight [26]. Finally, a specifier of "tic-related" OCD has been added [26].

While the most up-to-date DSM-5 diagnostic criteria are presented here, it is important to note that most of the treatment data described within this section are based on patients meeting DSM-IV criteria (or older).

\section{Psychological treatment}

Meta-analyses support the beneficial effects of psychological treatment for OCD, mainly CBT, generally including exposure with response prevention (ERP) [60-63,70,71, 655-657]. CBT is equivalent or superior to pharmacotherapy [71,658-660]. Results with CBT were generally similar in comparisons of interventions with an emphasis on ERP and those with an emphasis on cognitive elements $[60,63,655]$. A treatment specifically designed to address fear of contamination with infectious substances, using a cognitive intervention that includes no direct exposure ("danger ideation reduction therapy, DIRT"), was found to be more efficacious than ERP $[661,662]$. Cognitive interventions may be important in patients who do not have overt compulsions, which can make ERP more difficult. One meta-analysis found that exposure in vivo combined with imaginal exposure was better than exposure in vivo alone [60].

Several meta-analyses have demonstrated no significant differences in efficacy between group and individual CBT $[60,62,663]$. However, results of head-to-head trials are conflicting, with some RCTs finding no significant differences in efficacy between group and individual therapy $[663,664]$, and others showing individual therapy to be superior [665-667]. Differences in results may be explained by the fact that in individual therapy the therapist may have the advantage of being more aware of the patient's dysfunctional beliefs, however, the group therapy setting may offer the advantages of group encouragement, reciprocal support, imitation, and interpersonal learning which may result in an increased motivation and reduced discontinuation of treatment [62].

An important practical question concerns the intensity and duration of treatment. The intensive ERP program described by Foa's group involves 15 two-hour sessions scheduled five days a week over three weeks $[658,668]$. A similar program administered twice-weekly (a more practical approach for many patients and therapists) was as effective at the end of follow-up as the intensive fivedays/week strategy [669]. A step-care approach in which patients received six weeks of low-intensity counseling with ERP bibliotherapy followed by standard ERP for non-responders only was found to be as effective as initial therapy with standard ERP (17 sessions twice weekly), but was significantly less costly [670].

Other techniques that may be useful include acceptance and commitment therapy (ACT) [671], modular cognitive therapy (CT) addressing OCD beliefs [672,673], CT addressing obsessional doubt [674], organizational training [675,676], and mindfulness training [677]. RCTs on the benefits of adding motivational interviewing to CBT have been conflicting, with one showing no additional benefits [678], while another demonstrated improved symptom reduction and remission rates compared with CBT alone [679]. While EMDR was more effective than an SSRI in a RCT [680], data are limited and this technique is not generally recommended for patients with OCD.

Data suggest that therapist-guided exposure is better than self-exposure [60]. While both treatment conditions showed significant symptom reduction, therapistadministered ERP was superior to self-administered ERP in improving OCD symptoms and self-reported functional impairment [681]. Other data suggest that ERP 
delivered by telephone is equivalent to face-to-face ERP [682]. Bibliotherapy in the form of self-help manuals delivered to patients via email has demonstrated significantly greater improvements in OCD symptoms compared with wait-list control groups in two RCTs $[683,684]$.

ICBT is an easily accessible treatment that has the potential to reach untreated patients and motivate them for face-to-face psychotherapy if necessary [684,685]. Several RCTs have demonstrated that ICBT programs are significantly more effective than supportive therapy or relaxation control strategies [685-687]. ICBT was as effective as therapist-led CBT only when patients completed at least one self-exposure session [687]. ICBT was associated with significantly better outcomes when it included brief, scheduled, therapist-initiated telephone support compared with on-demand phone support [688].

Family accommodation (i.e., family members taking part in the performance of rituals, avoidance of anxietyprovoking situations, or modification of daily routines to assist a relative with OCD) has been associated with poorer response to both behavioral and pharmacological treatments [689]. Clinicians may want to consider targeting family accommodation in order to improve treatment outcomes for some patients.

Although hoarding disorder is now a separate diagnosis [690], the limited data available on the treatment of hoarding will be mentioned in this section on OCD. One RCT found that group CBT significantly reduced hoarding and depression symptoms while bibliotherapy alone was associated with very limited improvements [691]. The addition of posttreatment, nonclinician, home assistance did not significantly improve outcomes.

\section{Combined psychological and pharmacological treatment}

The combination of psychological and pharmacological treatment has been shown to be superior to medication alone $[657,658,692-694]$, but not to CBT alone $[83,658$, $692,694,695]$. These findings suggest that if pharmacotherapy is required or preferred, adding CBT to pharmacological treatment of OCD may enhance response rates and reduce relapse rates. Unlike in some anxiety and related disorders, there does not appear to be any contraindication to combining CBT with medications in patients with OCD [696], and combined treatment may improve relapse prevention [697].

Adding d-cycloserine may hasten the onset of improvements with ERP, with significant benefits over placebo during the first four or five ERP sessions [698-700], but this effect has not been seen in all studies [701].

\section{Long-term effects of psychological treatment}

Follow-up studies suggest that the benefits of CBT are maintained at one to five years of follow-up [664,695, 702-704].

\section{Pharmacological treatment}

The management of patients with OCD should follow the principles discussed in Section 2. SSRIs are recommended first-line pharmacological interventions for OCD, while SNRIs, clomipramine, and other antidepressants are recommended second- and third-line treatments. Treatments that have been investigated for use in OCD have been assessed according to the criteria for strength of evidence (Tables 1 and 2) and are summarized in Tables 26 and 27.

\section{First-line agents}

SSRIs: Evidence from RCTs and meta-analyses support the use of SSRIs, including escitalopram [705-709], fluoxetine [660,710-716], fluvoxamine [711,713,714,717-719], paroxetine [705,720-722], and sertraline [659,710,711, 713,714,723-725] (all Level 1), in the treatment of OCD. In meta-analyses, response rates with SSRIs are generally twice those of placebo [809], at 40-60\% with treatment versus $<20 \%$ with placebo [711,713,714,740,741]. Pooled response rates are not significantly different between SSRIs [809]. In meta-analyses and head-to-head trials, compared with clomipramine, the SSRIs fluoxetine, fluvoxamine, paroxetine, and sertraline had similar efficacy but better tolerability [711,713,714,716-718,720,724].

Dimensional analyses have suggested that symmetry/ hoarding symptoms may be associated with a poorer response to SSRI therapy [810,811], while aggressive/ religious/sexual symptoms may predict better outcomes $[810,812]$. It has been hypothesized that the symmetry/ hoarding symptom dimension may be mediated by the dopamine system and aggressive behaviors by the serotonin system [810,812].

\section{Second-line agents}

Clomipramine: There is good evidence to support the use of clomipramine in the treatment of OCD (Level 1) $[658,711,713,714,716-718,720,724,740,741]$. Clomipramine has efficacy similar to SSRIs, but SSRIs are generally better tolerated [711,713,714,716-718,720,724]. Side effects and safety are issues with clomipramine and therefore it is recommended as a second-line choice. Common adverse effects include anticholinergic effects such as dry mouth, constipation, and blurred vision, as well as urinary retention, orthostatic hypotension, weight gain, and sedation $[813,814]$. The major safety concerns are cardiac arrhythmias, seizures, drug interactions, and toxicity in overdose [813,814].

Antidepressants: In RCTs, citalopram was more effective than placebo but less effective than psychotherapy (Level 2) [680,726]. Additional data from augmentation studies support the efficacy of citalopram for the treatment of OCD $[727,728]$. However, given that other SSRIs have much stronger evidence, citalopram was designated a second-line option. The only RCT data on 
Table 26 Strength of evidence of pharmacotherapy for OCD

\begin{tabular}{|c|c|c|c|}
\hline Agent & Level of evidence & Agent & Level of evidence \\
\hline \multicolumn{4}{|l|}{ Antidepressants } \\
\hline SSRIs & & MAOIs & \\
\hline Escitalopram [705-709] & 1 & Phenelzine $[737,738]$ & $2^{*}$ \\
\hline Fluoxetine $[660,710-716]$ & 1 & Tranylcypromine [739] & 4 \\
\hline Fluvoxamine $[711,713,714,717-719]$ & 1 & TCAs & \\
\hline Paroxetine $[705,720-722]$ & 1 & Clomipramine $[658,711,713,714,716-718,720,724,740,741]$ & 1 \\
\hline Sertraline $[659,710,711,713,714,723-725]$ & 1 & IV clomipramine [742-744] & 2 \\
\hline Citalopram $[680,726-728]$ & 2 & Desipramine $[723,745]$ & $2(-v e)$ \\
\hline IV citalopram [729] & 3 & Adjunctive clomipramine $[746,747]$ & $2(-v e)$ \\
\hline Adjunctive citalopram [730] & 3 & Other antidepressants & \\
\hline SNRIs & & Mirtazapine [748] & 2 \\
\hline Venlafaxine XR [721,731-733] & 2 & Bupropion [749] & $3(-v e)$ \\
\hline Duloxetine [734-736] & 4 & Adjunctive mirtazapine [727] & 3 \\
\hline \multicolumn{4}{|l|}{ Other therapies } \\
\hline Antipsychotics & & Anxiolytics & \\
\hline Adjunctive aripiprazole [750-755] & 1 & Benzodiazepines & \\
\hline Adjunctive risperidone [755-761] & $1^{*}$ & Clonazepam [771] & $2(-v e)$ \\
\hline Adjunctive olanzapine $[760,762,763]$ & $1^{*}$ & Adjunctive clonazepam [772] & $2(-v e)$ \\
\hline Adjunctive quetiapine $[728,746,747,764-768]$ & $1^{*}$ & Other treatments & \\
\hline Adjunctive haloperidol $[758,769]$ & 2 & Clonidine [773] & $2(-v e)$ \\
\hline Adjunctive amisulpride [770] & 3 & Adjunctive pindolol [774-776] & $1^{*}$ \\
\hline Adjunctive ziprasidone [767] & 4 & Adjunctive celecoxib [777] & 2 \\
\hline Anticonvulsants & & Adjunctive granisetron [778] & 2 \\
\hline Adjunctive topiramate [795-798] & $1^{*}$ & Adjunctive IV ketamine $[779,780]$ & 2 \\
\hline Adjunctive lamotrigine $[799,800]$ & 2 & Adjunctive memantine $[622,781-783]$ & 2 \\
\hline Adjunctive pregabalin $[801,802]$ & 3 & Adjunctive ondansetron $[784,785]$ & 2 \\
\hline Adjunctive gabapentin $[803,804]$ & 3 (-ve) & Adjunctive N-acetylcysteine $[786,787]$ & 2 \\
\hline Opioids & & Adjunctive riluzole $[788,789]$ & 3 \\
\hline Tramadol $[805,806]$ & 4 & Adjunctive lithium $[790,791]$ & 1 (-ve) \\
\hline Naltrexone [807] & $3(-v e)$ & Adjunctive buspirone $[792,793]$ & $2(-v e)$ \\
\hline Adjunctive morphine [808] & 2 & Adjunctive minocycline [794] & $4(-v e)$ \\
\hline
\end{tabular}

*Conflicting data. IV = intravenous; MAOI = monoamine oxidase inhibitor; SNRI = serotonin-norepinephrine reuptake inhibitor; SSRI = selective serotonin reuptake inhibitor; TCA = tricyclic antidepressant; $X R=$ extended release; $(-v e)=$ negative.

the use of mirtazapine in OCD are from a discontinuation study in which continued mirtazapine was associated with continued improvement (Level 2) [748]. There is some evidence to support the use of venlafaxine XR for the treatment of OCD (Level 2) [721,731-733]. In RCTs, venlafaxine XR was more effective than placebo [732], and as effective as paroxetine [721] and clomipramine [731]. In a double-blind extension of a RCT [721],

Table 27 Recommendations for pharmacotherapy for OCD

\begin{tabular}{ll}
\hline First-line & Escitalopram, fluoxetine, fluvoxamine, paroxetine, sertraline \\
\hline Second-line & Citalopram, clomipramine, mirtazapine, venlafaxine XR \\
\hline Third-line & IV citalopram, IV clomipramine, duloxetine, phenelzine, tramadol, tranylcypromine \\
\hline $\begin{array}{l}\text { Adjunctive } \\
\text { therapy }\end{array}$ & $\begin{array}{l}\text { First-line: aripiprazole, risperidone } \\
\text { Second-line: memantine, quetiapine, topiramate } \\
\text { Third-line: amisulpride, celecoxib, citalopram, granisetron, haloperidol, IV ketamine, mirtazapine, N-acetylcysteine, olanzapine, } \\
\text { ondansetron, pindolol, pregabalin, riluzole, ziprasidone } \\
\text { Not recommended: buspirone, clonazepam, lithium, morphine }\end{array}$ \\
\hline Not & Clonazepam, clonidine, desipramine \\
\hline
\end{tabular}

IV = intravenous; $\mathrm{XR}=$ extended release. 
paroxetine was more efficacious than venlafaxine in the treatment of non-responders to previous treatment with the alternate antidepressant [733].

\section{Third-line agents}

Intravenous clomipramine: In a RCT, intravenous (IV) clomipramine was more effective than placebo in patients with OCD (Level 2) [742]. Initiating therapy with IV then switching to oral therapy does not appear to be associated with greater benefit compared with oral therapy alone [743,744].

Other agents: IV citalopram [729] (Level 3), as well as duloxetine [734-736], tramadol [805,806], and tranylcypromine [739] (all Level 4) have demonstrated some efficacy in open trials or case reports. Results with phenelzine have been inconsistent. In one RCT, phenelzine was not significantly better than placebo [738], but in another it was as effective as clomipramine (Level 2) [737]. In the placebo-controlled trial, post-hoc analysis suggested that phenelzine may be beneficial in patients with symmetry or other atypical obsessions [738].

These agents are recommended as third-line options, and may be useful in refractory patients after first- and second-line monotherapies and adjuncts have been unsuccessful.

\section{Adjunctive therapy}

Adjunctive strategies have generally been studied in patients who have had an inadequate response to SSRI therapy, and can be considered for patients with treatment-resistant OCD. A meta-analysis demonstrated that response rates with adjunctive medication were twice those of placebo, however these were still quite low (31.8\% versus $13.6 \%$ ) [815]. Meta-analyses of RCTs found that adding risperidone (and possibly quetiapine) to antidepressants increased efficacy but decreased tolerability, while adjunctive olanzapine did not improve response rates $[816,817]$.

First-line adjunctive therapies: In RCTs, adjunctive aripiprazole was significantly more effective than placebo (Level 1) [750,754], and may be as effective as risperidone [755]. Additional open-label data also support the beneficial effects of adjunctive aripiprazole [751-753].

As adjunctive therapy for treatment-resistant OCD, risperidone was more effective than placebo (Level 1) [756-759] and as effective as olanzapine [760] and aripiprazole overall [755]. Compared with aripiprazole, risperidone may provide greater improvement in obsessions [755]. Risperidone was also as effective as haloperidol for obsessions, but less so for compulsions, however it was better tolerated [758]. More recently an open, randomized study found that while augmentation with ERP was superior to risperidone or pill placebo, risperidone was not significantly more effective than placebo [761]. However, patients in this study had some response to SSRI therapy and may have been less refractory compared to those in other studies. Considering the tolerability concerns of atypical antipsychotics, these data reinforce that this augmentation strategy should be reserved for patients with treatment-resistant OCD.

Second-line adjunctive therapies: RCT evidence demonstrated that adjunctive memantine was superior to placebo (Level 2) [783]. Additional open-label data also support this therapy [622,781,782]. Another option which may be useful as an adjunctive therapy in those with refractory OCD is the atypical antipsychotic quetiapine (Level 1, conflicting) [728,746,747,764-766,768].

Data from small RCTs suggest that topiramate may be a useful adjunctive therapy, but data are conflicting (Level 1) [796,797]. In one RCT, adjunctive topiramate significantly improved Yale-Brown Obsessive Compulsive Scale (Y-BOCS) scores compared with placebo [797], while in another trial, adjunctive topiramate significantly improved compulsions but not obsessions [796]. Additional open-label data support the use of adjunctive topiramate [795,798].

Third-line adjunctive therapies: The agents discussed below are recommended as third-line adjunctive options, since some data are available to suggest they may be useful but there is conflicting or inadequate evidence to warrant stronger recommendations. These agents may be useful for some patients, but more data are needed.

Other atypical antipsychotics have been assessed as adjunctive therapies in patients with refractory OCD, including olanzapine (Level 1, conflicting) [760,762,763], amisulpride (Level 3) [770], and ziprasidone (Level 4) [767].

There is level 2 evidence to support the use of adjunctive haloperidol in patients with refractory OCD $[758,769]$, and although it may be as effective as adjunctive risperidone, it is a third-line choice because it was less well tolerated [758].

Adjunctive mirtazapine was associated with an earlier onset of response of OCD symptoms compared with citalopram alone, but there was no advantage of the combination over time (Level 2) [727]. Some data also support the efficacy of adjunctive citalopram for treatmentresistant OCD (Level 3) [730].

Adjunctive anticonvulsants may be useful for some patients with refractory illness [799-802]. In a small RCT, adjunctive lamotrigine improved both obsessions and compulsions compared to SSRI therapy (Level 2) [799]. Open-label data also suggest that adjunctive pregabalin may be useful (Level 3) [801,802].

Other agents that have been studied as adjunctive therapy for treatment-resistant OCD include celecoxib [777], granisetron [778], IV ketamine [779,780], ondansetron [784,785], N-acetylcysteine [786,787] (all Level 2), and riluzole (Level 3) [788,789]. There is little clinical experience with these agents for refractory OCD, 
therefore they are recommended as third-line adjunctive options only.

Results with pindolol augmentation have been inconsistent, with significant improvements in one small RCT [774], but not in other randomized or open trials (Level 1, conflicting) [775,776].

In two randomized, quetiapine-controlled trials, adjunctive clomipramine was not superior to SSRI therapy (Level 2, negative) [746,747]. Clinical experience suggests that some patients may benefit from adjunctive clomipramine; however, plasma levels should be monitored because of the risk of drug interactions with SSRIs $[747,813]$.

\section{Not recommended}

Clonazepam [771], clonidine [773], and desipramine (all Level 2, negative) [723,745] have not demonstrated efficacy and are not recommended in the treatment of OCD. Bupropion [749] and naltrexone (both Level 3, negative) [807] also do not appear to be effective in this disorder.

Adjunctive buspirone [792,793], clonazepam [772] (Level 2, negative), or lithium [790,791] (Level 1, negative) have not demonstrated efficacy for the treatment of OCD. There is currently no evidence for the efficacy of adjunctive gabapentin (Level 3, negative) $[803,804]$ or minocycline (Level 4, negative) [794], but there are insufficient data to make recommendations at this time. In a $\mathrm{RCT}$, adjunctive once-weekly oral morphine was effective in patients who had failed six SSRI trials (Level 2) [808], however, morphine is not generally recommended because of its potential for abuse.

\section{Maintenance pharmacological treatment}

Long-term therapy has been evaluated in relapse prevention and naturalistic follow-up studies. Relapse-prevention studies are those in which responders to SSRI therapy are randomized to continued active treatment or placebo. A meta-analysis of six relapse prevention studies included 951 patients with OCD and found a highly significant reduction in relapse rates with continued SSRI treatment compared with placebo over six to 12 months (odds ratio for relapse was 0.38) [497]. In RCTs, escitalopram [818], paroxetine [722], sertraline [819], and high-dose fluoxetine [820] have demonstrated reductions in relapse rates. In RCT discontinuation studies, mirtazapine [748] and clomipramine [821] have demonstrated continued improvement compared with placebo over approximately six to 12 months. Additional data support the long-term efficacy of fluoxetine, fluvoxamine XR, and sertraline over six to 24 months $[710,822-824]$.

\section{Biological and alternative therapies}

Biological therapies: Biological therapies may be useful in patients with OCD who have not responded to CBT and multiple medication trials. Open trials have suggested that rTMS may be a promising adjunctive therapy in patients with treatment-refractory OCD $[825,826]$. However, results of sham-controlled trials are conflicting, with some trials finding significant improvements $[827,828]$ and others concluding that rTMS was ineffective for treatment-resistant OCD (Level 1, conflicting) [829-831]. Some data suggest that rTMS may improve comorbid depressive symptoms in patients with OCD $[829,830]$.

Several very small studies have suggested that deep brain stimulation may improve symptoms and functionality in up to two-thirds of patients with highly treatment-refractory OCD (Level 4) [832-834].

Open trials suggest that capsulotomy (Level 3) [835-839] or cingulotomy (Level 3) [840-842] may be effective in reducing symptoms in patients with severe, treatment-refractory OCD, however these treatments are usually considered last resorts.

Alternative therapies: A meta-analysis of meditation therapies found only two small studies and showed that transcendental meditation and Kundalini yoga were likely no more effective than other kinds of relaxation therapies in treating OCD (Level 3, negative) [843]. Open studies suggest that adjunctive moderate-intensity aerobic exercise may help improve OCD symptoms (Level 3) [844,845].

Small RCTs and open trials have suggested that herbal therapies such as milk thistle (Silybum marianum L. Gaertn.) (Level 2) [715], valerian root (Valeriana officinalis L.) (Level 2) [846], and St John's wort (Hypericum perforatum) (Level 3) [847] may be useful in patients with OCD. Unfortunately, because these preparations are poorly standardized and have substantial variation in the proportion of the active ingredient in different products, they cannot be widely recommended. These therapies may be useful for some patients; however, more data are needed.

\section{Summary}

OCD is a relatively rare, yet severe, mental disorder, with an onset in the 20 s or earlier. It is characterized by the presence of obsessions (persistent, intrusive thoughts) and/or compulsions (repetitive behaviors the individual feels compelled to perform). OCD is associated with substantial functional impairment and a high prevalence of comorbid disorders.

CBT, and notably ERP, are effective first-line options for the treatment of OCD, being equivalent or superior to pharmacotherapy. CBT can be effectively delivered in both individual and group settings, as well as via self-exposure, self-help books, telephone, and internet-based programs. The benefits of CBT are maintained over one to five years of follow-up. The combination of psychotherapy and pharmacotherapy appears to be superior to pharmacotherapy 
alone, but not to CBT alone, and data suggest that adding CBT to pharmacological treatment may yield better longterm outcomes.

Pharmacotherapeutic approaches should begin with a first-line SSRI such as escitalopram, fluoxetine, fluvoxamine, paroxetine, or sertraline. If response to optimal doses is inadequate or the agent is not tolerated, therapy should be switched to another first-line agent before considering second-line medications. Second-line choices include citalopram, clomipramine, mirtazapine, and venlafaxine XR. OCD can be difficult to treat; therefore, in order to preserve any benefits of a therapy, adjunctive strategies may be important early in treatment.

Patients who do not respond to multiple courses of therapy are considered to have treatment-refractory illness. In such patients it is important to reassess the diagnosis and consider comorbid medical and psychiatric conditions that may be affecting response to therapy. Third-line agents, adjunctive therapies, as well as biological and alternative therapies may be useful when patients fail to respond to optimal treatment trials of first- and second-line therapies used alone and in combination.

\section{Postraumatic stress disorder Epidemiology}

The lifetime prevalence of PTSD in Canada was estimated to be $9.2 \%$, and current (1-month) rates were $2.4 \%$ [848]. Over $76 \%$ of Canadians reported exposure to a significantly traumatic event [848]. US and European community studies report lifetime prevalence rates of 6.4-6.8\% and 12-month rates of $1.1-3.5 \%$ [2,849,850]. The most common forms of trauma resulting in PTSD included unexpected death of someone close, sexual assault, serious illness or injury to someone close, having a child with serious illness, and being beaten by a partner or caregiver [848-850]. Onset is generally in the mid to late 20s [2], and the prevalence is about twice as high among women versus men $[849,851]$.

PTSD was associated with significant QoL [852] and functional impairments [848,853-855], which increase with increasing severity of symptoms [855]. In addition, PTSD is associated with high rates of chronic pain [856-859], sleep problems [860], and sexual dysfunction [861], as well as cognitive dysfunction [862,863] and alexithymia [864]. The risk of suicide attempts is increased two- to three-fold by the presence of PTSD $[20,849,865]$.

In primary care, PTSD was associated with more and longer hospitalizations as well as a greater use of mental health care [866]. Among Canadian military personnel, greater use of mental health care was associated with cumulative lifetime trauma exposure, index trauma type, PTSD symptom interference, suicidal ideation, female gender, and comorbid MDD [867,868].

\section{Comorbidity}

An estimated $75 \%$ of patients with PTSD have another comorbid psychiatric disorder [3,848]; and rates are particularly high for other anxiety and related disorders $[3,849,859,869,870]$, MDD [3,849,859,871,872], oppositional defiant disorder [3], ADHD [3], SUD [849], alcohol dependence $[3,873]$, and borderline personality disorder (BPD) [874]. Comorbid panic or mood disorders have been associated with greater functional impairment than PTSD alone [870,871]. Patients with comorbid PTSD and BPD had a poorer QoL, more comorbidity with other psychiatric conditions, and increased odds of a lifetime suicide attempt versus patients with either condition alone [20,874].

\section{Diagnosis}

By definition PTSD requires exposure to trauma, including actual or threatened death, serious injury, or sexual violation [26]. It is characterized by intrusive and distressing memories or dreams, dissociative reactions, and substantial psychological or physiological distress related to the event (Table 28) [26]. A diagnosis of PTSD requires the disturbances to be present for longer than one month; symptoms of $>3$ days but less than one month may be diagnosed as acute stress disorder (ASD), if the required ASD criteria are met [26].

Compared to the DSM-IV-TR [144], changes to the diagnostic criteria for PTSD in the DSM-5 include adjusting the symptom clusters, adding some new symptoms, and re-classifying PTSD as a "trauma- and stressor-related disorder" instead of an anxiety disorder [26,875]. In addition to PTSD, this new category also includes diagnostic criteria for reactive attachment disorder, disinhibited social engagement disorder, ASD, and adjustment disorders [26]. The DSM-5 diagnostic criteria for PTSD sharpens the definition of "traumatic event," and there are now four symptom clusters rather than three with the "avoidance" and "numbing of responsiveness" being separated (Table 28). The DSM-5 also eliminated the acute and chronic PTSD specifiers. The PTSD diagnostic criteria apply to adults, adolescents, and children $>6$ years of age. A subtype has been added for children $\leq 6$ years of age, as well as a dissociative symptoms specifier for patients of all ages [26].

While the most up-to-date DSM-5 diagnostic criteria are being presented here, it is important to note that the treatment data described within this section are based on patients meeting DSM-IV criteria (or older).

PTSD is frequently comorbid with other psychiatric disorders, including other anxiety and related disorders, MDD, and SUDs, which may complicate diagnosis and management $[849,859]$. In addition, patients with PTSD frequently present with somatic symptoms and pain [859]. It is important to ask patients with psychological or somatic symptoms about trauma [32,859]. 


\section{Table 28 DSM-5 diagnosis of PTSD}

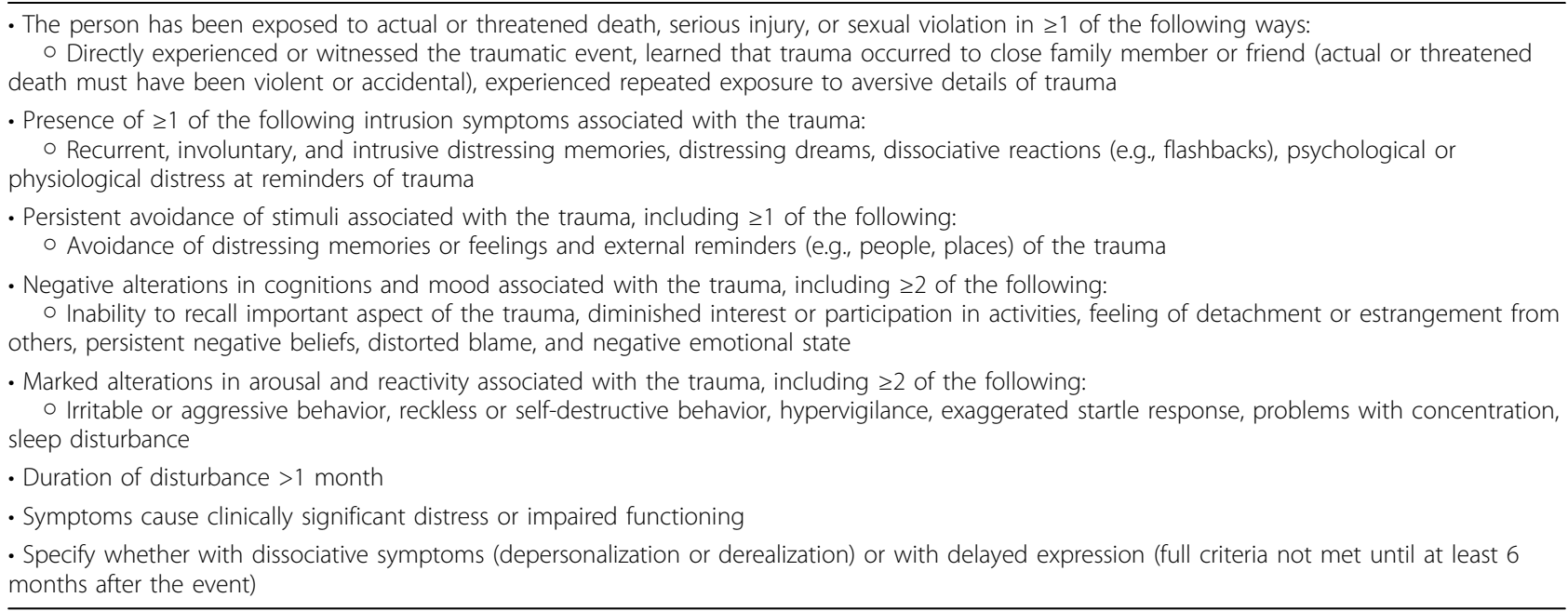

Adapted from DSM-5 [26].

\section{Prevention and early intervention}

A number of studies have assessed early intervention with psychological and pharmacological strategies for the prevention of PTSD. Meta-analyses do not support the efficacy of wide spread use of single-session [876,877] or multiple-session [878] psychological debriefing after trauma in preventing or reducing the intensity of PTSD in individuals who have been exposed to a traumatic event but have not been identified as suffering from any specific psychological difficulties. In fact, these interventions may have an adverse effect on some individuals $[876,878]$. These findings pertain to individual debriefings only; there is insufficient evidence to comment on the utility of group debriefings.

Conversely, meta-analyses have demonstrated the benefit of multisession trauma-focused-CBT (TF-CBT) in patients with ASD or PTSD $[879,880]$. Therefore, debriefing of all trauma victims is not recommended, rather, screening and treating appropriate individuals is preferred [876]. For the prevention of chronic PTSD in patients with ASD or acute PTSD, brief TF-CBT was more effective than both wait-list and supportive counseling interventions, but there was no evidence of the effectiveness of structured writing compared to minimal intervention [880].

There are few data on the use of pharmacotherapy for the prevention of PTSD. In a cohort study and a RCT, the early use of benzodiazepines following trauma was not beneficial, and may increase the risk of developing PTSD $[881,882]$. Similarly, retrospective data suggested that gabapentin or pregabalin had no effect on PTSD development [883]. Data from cohort studies on the use of the beta-blocker propranolol have been conflicting [884-888], but one small RCT did show a significant decrease in the severity of PTSD symptoms and lower likelihood of developing subsequent PTSD [889]. SSRI therapy was significantly more effective than placebo in preventing PTSD symptoms according to parent reports but not child reports in a RCT in children [890]. Cohort studies suggest that the early use of morphine during trauma care may reduce the risk of the subsequent development of PTSD in children and adults [891-894].

\section{Psychological treatment}

Psychological therapies for PTSD generally include education about the disorder and its treatment, as well as exposure to cues relating to the traumatic event. Psychotherapy has demonstrated significant efficacy, although a metaanalysis suggested it may be less effective than pharmacotherapy in improving PTSD and comorbid depression symptoms [895].

Meta-analyses of over 30 RCTs of psychological interventions provide evidence of the efficacy of several CBT approaches for the management of chronic PTSD compared with wait-list or usual care control groups [66,67]. There was evidence that individual TF-CBT, EMDR, stress management, and group TF-CBT were effective, while other nontrauma focused psychological treatments (supportive therapy, nondirective counseling, psychodynamic therapy, and hypnotherapy) did not reduce PTSD symptoms as significantly $[66,67]$. Individual TF-CBT and EMDR appeared to be equally effective, but superior to stress management in the treatment of PTSD [66]. Another meta-analysis also found EMDR and TF-CBT were equally effective [68]. However, in a head-to-head RCT, EMDR resulted in faster recovery compared with the more gradual improvement with brief TF-CBT [896]. Cognitive therapy approaches have been used effectively in treating PTSD following sexual or interpersonal violence [897-901], civilian trauma [902-908], and military trauma [909-914]. 
Cognitive processing therapy (CPT) is an effective protocol that combines cognitive therapy and written accounts [899-901,910-913]; however, an analysis of the components found no differences in outcomes with either component alone or the combined protocol [899].

Prolonged exposure (PE) is a widely studied CBT approach. A meta-analysis of 13 RCTs concluded that PE therapy was more effective than wait-list or psychological placebo control conditions, and as effective as other active treatments (e.g., CBT, CPT, EMDR) [69]. One study found that 30-minute imaginal exposure sessions were as effective as 60-minute sessions [915]. Imaginal appears to be as effective as in vivo exposure [69,916].

Data are conflicting as to the benefits of adding cognitive restructuring to exposure therapy; several studies suggest that exposure alone is superior to the combination [917-919], however, another large RCT found the combination to be significantly better than imaginal or in vivo exposure alone [916]. When used as an adjunct to exposure therapy, cognitive restructuring may improve non-fear problems like anger and guilt, and may be a useful adjunct in patients in which these emotions predominate $[920,921]$. Similarly, the addition of social emotional rehabilitation to exposure therapy did not improve PTSD symptoms but did improve social functioning in male combat veterans with chronic PTSD [922].

Meta-analyses and systematic reviews reveal two current limitations of CBT for PTSD. The first is that about one-third to one-half of patients experience substantial residual symptoms and functional impairments posttreatment, still report symptoms meeting diagnostic criteria at follow-up, or relapse and require booster sessions [923-925]. The second issue pertains to external validity. While CBT for PTSD has been shown to be efficacious in RCTs, there is a dearth of effectiveness studies to suggest that CBT can be generalized to many patients commonly found in clinical practice. Many RCTs have excluded patients with complex clinical profiles including childhood abuse histories, current SUDs, personality disorders, suicidality or self-injurious behavior, homelessness, refugees, intimate partner violence, and significant dissociative symptoms among others $[926,927]$. In this regard, Bradley et al. [923] found a positive association between the number of exclusion criteria and the strength of effect sizes, such that studies with stricter inclusion criteria tended to report larger treatment effects. Additionally, numerous studies fail to report whether patients experience any adverse effects from psychological treatments [66], or whether dropout rates (ranging between 0-50\%) result from treatment demands.

Dialectical behavior therapy (DBT), which was developed to reduce self-harm behavior in patients with BPD, was shown to be useful in patients with PTSD [928-930]. When used as a pretreatment, DBT reduced self-harm behaviors allowing over half of patients to become suitable candidates for PTSD treatment [929].

Another study [931] demonstrated some success with PE treatment of PTSD and comorbid substance abuse. Results of a recent expert clinician survey on best practices suggests that CBT is useful for fear-based PTSD, while this treatment approach may require an additional treatment module targeting affective regulation for patients presenting with a diagnosis of Disorders of Extreme Stress (DESNOS) or complex PTSD [932].

Internet-based treatments are being increasingly investigated, in part because they can be administered remotely and anonymously to under-served or disaster-stricken areas at a relatively low-cost [933]. RCTs have shown that therapist-assisted ICBT is more effective than wait-list or supportive care control strategies in improving PTSD symptoms, depression, anxiety, and disability [934-940]. In addition, a strong therapeutic relationship can be established through the internet, which improved the treatment process [936]. VRE therapy has also demonstrated some utility in improving PTSD symptoms [941-943]. Compared to face-to-face CBT, video-conference CBT was equally effective [944] but telehealth CBT was less effective [914]; however, both were effective compared with pre-treatment.

\section{Combined psychological and pharmacological treatment}

Research evaluating combined treatment in PTSD is limited; a meta-analysis found only four small trials [945]. Combination SSRI plus psychotherapy was not superior to psychotherapy alone in two RCTs $[946,947]$, but was superior to pharmacotherapy alone in the other two trials $[948,949]$. In contrast, a more recent RCT found that combination therapy was superior to psychotherapy alone [950]. The role of combining psychotherapy and medication requires further study.

Adjunctive propranolol with trauma reactivation therapy was found to help prevent reconsolidation of the traumatic memory and thus decreased physiological responses and PTSD symptoms during subsequent follow-up in randomized and open trials [951,952]. Two RCTs have found that use of d-cycloserine did not enhance the overall treatment effects of exposure therapy [953,954], and may in fact decrease response to psychotherapy [954].

\section{Long-term effects of psychological treatment}

Open follow-up data of psychological treatments suggest that benefits are maintained at six- to 18-month assessments after treatment [923,955-958]. Longer-term follow-up of patients treated with EMDR showed that benefits were maintained at three years, with the majority of patients who had initially remitted being at full working capacity [959]. Very long-term follow-up showed that improvements in PTSD and related symptoms achieved with CPT and PE were maintained over an extended five to 10 year period [901]. 


\section{Pharmacological treatment}

The management of patients with PTSD should follow the principles discussed in Section 2. Pharmacological interventions that have good evidence for efficacy in treating PTSD include fluoxetine, paroxetine, sertraline, and venlafaxine XR. Treatments that have been investigated for use in PTSD have been assessed according to the criteria for strength of evidence (Tables 1 and 2) and are summarized in Tables 29 and 30.

\section{First-line agents}

Antidepressants (SSRIs E SNRIs): Evidence from metaanalyses [895,1060] and RCTs supports the use of the SSRI paroxetine [966-970] and the SNRI venlafaxine XR [975,989] (both Level 1) for the first-line treatment of
PTSD. Data with fluoxetine are mixed, with both positive [960-962] and negative [963-965] RCTs (Level 1, conflicting). Similarly, RCTs with sertraline have yielded both positive $[971,972,975,976,978]$ and negative [973,974,977] results (Level 1, conflicting). However, there appear to be sufficient data from the larger RCTs to suggest that these agents can be effective first-line options. Conflicting results may be related to the types of traumas, symptom clusters, and comorbidities included in the various studies.

\section{Second-line agents}

Antidepressants: The efficacy of mirtazapine was demonstrated in one small RCT (Level 2) [1000] and three open trials $[999,1001,1002]$. In a randomized, open-label

Table 29 Strength of evidence of pharmacotherapy for core symptoms of PTSD

\begin{tabular}{|c|c|c|c|}
\hline Agent & Level of evidence & Agent & Level of evidence \\
\hline \multicolumn{4}{|l|}{ Antidepressants } \\
\hline SSRIs & & TCAs & \\
\hline Fluoxetine [960-965] & $1^{*}$ & Imipramine [992,993] & 1 \\
\hline Paroxetine [966-970] & 1 & Amitriptyline [994] & 2 \\
\hline Sertraline [971-978] & $1^{*}$ & Desipramine $[970,995]$ & $2^{*}$ \\
\hline Fluvoxamine [979-984] & 2 & MAOIs and RIMAs & \\
\hline Escitalopram [985] & 3 & Phenelzine $[992,993,996]$ & $1^{*}$ \\
\hline Citalopram [974,986,787,988] & 2 (-ve) & Moclobemide $[997,998]$ & 3 \\
\hline SNRIs & & Other antidepressants & \\
\hline Venlafaxine XR $[975,989]$ & 1 & Mirtazapine [999-1002] & 2 \\
\hline \multirow[t]{4}{*}{ Duloxetine $[990,991]$} & 3 & Reboxetine [984] & 2 \\
\hline & & Bupropion SR [1003] & 3 \\
\hline & & Tianeptine $[997,1004]$ & 3 \\
\hline & & Adjunctive bupropion SR [1005] & $2(-v e)$ \\
\hline \multicolumn{4}{|l|}{ Other therapies } \\
\hline Anxiolytics & & Anticonvulsants & \\
\hline Benzodiazepines & & Topiramate $[1009,1010]$ & $1^{*}$ \\
\hline Alprazolam [1006] & $2(-v e)$ & Lamotrigine [1011] & 2 \\
\hline Clonazepam [881,1007,1008] & 3 (-ve) & Carbamazepine $[1012,1013]$ & 3 \\
\hline Atypical antipsychotics & & Divalproex [1014-1017] & 1 (-ve) \\
\hline Risperidone [1030] & 2 & Tiagabine [1018] & $2(-v e)$ \\
\hline Aripiprazole [1031-1033] & 3 & Adjunctive gabapentin $[1019,1020]$ & 4 \\
\hline Quetiapine $[1034,1035]$ & 3 & Adjunctive levetiracetam [1021] & 4 \\
\hline Olanzapine [1036-1038] & $2(-v e)$ & Adjunctive pregabalin [1022] & 4 \\
\hline Adjunctive risperidone [1039-1044] & $1^{*}$ & Adjunctive tiagabine [1023-1025] & 4 \\
\hline Adjunctive olanzapine [1045] & 2 & Adjunctive topiramate [1026-1029] & $2(-v e)$ \\
\hline Adjunctive aripiprazole $[1033,1046,1047]$ & 3 & Other treatments & \\
\hline \multirow[t]{7}{*}{ Adjunctive quetiapine [1048-1050] } & 3 & Buspirone $[1051,1052]$ & 4 \\
\hline & & Trazodone [1053] & 4 \\
\hline & & Memantine [1054] & 4 \\
\hline & & Adjunctive eszopiclone [1055] & 2 \\
\hline & & Adjunctive clonidine [1056] & 3 \\
\hline & & Adjunctive guanfacine $[1057,1058]$ & 1 (-ve) \\
\hline & & Adjunctive zolpidem [1059] & $2(-v e)$ \\
\hline
\end{tabular}

*Conflicting data. $\mathrm{MAOI}=$ monoamine oxidase inhibitor; RIMA = reversible inhibitor of monoamine oxidase A; SNRI = serotonin-norepinephrine reuptake inhibitor; $\mathrm{SR}=$ sustained release; $\mathrm{SSRI}=$ selective serotonin reuptake inhibitor; $\mathrm{TCA}=$ tricyclic antidepressant; $\mathrm{XR}=\mathrm{extended}$ release; $(-\mathrm{ve})=$ negative . 
Table 30 Recommendations for pharmacotherapy for core symptoms of PTSD

\begin{tabular}{ll}
\hline First-line & Fluoxetine, paroxetine, sertraline, venlafaxine XR \\
\hline Second-line & Fluvoxamine, mirtazapine, phenelzine \\
\hline Third-line & Amitriptyline, aripiprazole, bupropion SR, buspirone, carbamazepine, desipramine, duloxetine, escitalopram, imipramine, \\
& lamotrigine, memantine, moclobemide, quetiapine, reboxetine, risperidone, tianeptine, topiramate, trazodone \\
\hline $\begin{array}{l}\text { Adjunctive } \\
\text { therapy }\end{array}$ & $\begin{array}{l}\text { Second-line: eszopiclone, olanzapine, risperidone } \\
\text { Third-line: aripiprazole, clonidine, gabapentin, levetiracetam, pregabalin, quetiapine, reboxetine, tiagabine } \\
\text { Not recommended: bupropion SR, guanfacine, topiramate, zolpidem }\end{array}$ \\
\hline $\begin{array}{l}\text { recommended } \\
\text { NR }\end{array}$ & Alprazolam, citalopram, clonazepam, desipramine, divalproex, olanzapine, tiagabine \\
\hline
\end{tabular}

$\mathrm{SR}=$ sustained release; $\mathrm{XR}=$ extended release.

trial, response rates were significantly higher with mirtazapine than sertraline [1001].

Fluvoxamine demonstrated efficacy for PTSD in open trials [979-983], and in a RCT was as effective as reboxetine (Level 2) [984].

Phenelzine was more effective than placebo in two RCTs [992,993], but not significantly different from placebo in a RCT crossover study (Level 1, conflicting) [996]. Caution is needed when using MAOIs because of the dietary restrictions and potential for drug interactions.

\section{Third-line agents}

The following agents are recommended as third-line options because of limited data, side effects, or lack of clinical experience as a primary therapy for the treatment of PTSD.

Antidepressants: In small RCTs, imipramine (Level 1) [992,993] and amitriptyline (Level 2) [994] demonstrated some efficacy in patients with PTSD. Data with desipramine are mixed, with one RCT showing significant benefit, which were comparable to paroxetine [970], and the other showing improvements in depression only [995]. While RCTs with the TCAs suggest some benefit with these agents, it appears to be limited.

Reboxetine and fluvoxamine were equally effective in a small RCT (both Level 2) [984], and open-label studies suggest that bupropion SR [1003], duloxetine [990,991], escitalopram [985], moclobemide [997,998], and tianeptine $[997,1004]$ (all Level 3) may be useful in PTSD.

Anticonvulsants: Data on topiramate are mixed, with one RCT finding significant benefits over placebo [1010], while the other did not [1009] (Level 1, conflicting). There are also limited data suggesting efficacy of other anticonvulsants, including lamotrigine (Level 2) [1011] and carbamazepine (Level 3) [1012,1013].

Atypical antipsychotics: Some data suggest that the atypical antipsychotics, risperidone (Level 2) [1030], aripiprazole (Level 3) [1031,1032], and quetiapine (Level 3) [1034,1035] may be a useful alternative to SSRIs for some patients with PTSD. A meta-analysis of seven RCTs using atypical antipsychotics, either as monotherapy or adjunctively, concluded that these agents may be beneficial in the treatment of PTSD, particularly for the symptom of "intrusion" [1061].
Other therapies: Small, open case series have suggested benefits with trazodone [1053], buspirone [1051,1052], and memantine [1054] (all Level 4).

\section{Adjunctive therapies}

Adjunctive strategies have generally been studied in patients who have had an inadequate response to adequate antidepressant therapy, and can be considered for patients with treatment-resistant PTSD.

Second-line adjunctive therapies: In a RCT, adjunctive eszopiclone was significantly more effective than placebo in improving PTSD and sleep symptoms (Level 2) [1055]. There is RCT evidence for the use of adjunctive atypical antipsychotics, including risperidone (Level 1, conflicting) [1039-1044] and olanzapine (Level 2) [1045], for patients with treatment-resistant PTSD. While a number of small RCTs demonstrated benefits with adjunctive risperidone [1039-1042], a large, six-month trial in approximately 250 patients failed to show improvements in PTSD symptoms compared with placebo [1043].

Third-line adjunctive therapies: Open-label trials and case series suggest that adjunctive quetiapine [1048-1050] or aripiprazole [1033,1046,1047] (both Level 3) are useful in patients with refractory PTSD.

Similarly, there are some data suggesting adjunctive anticonvulsants including: gabapentin [1019,1020], levetiracetam [1021], pregabalin [1022], or tiagabine [1023-1025] (all Level 4), as well as the alpha-adrenergic agonist clonidine (Level 3) [1056], can improve symptoms in patients with treatment-resistant PTSD.

Not recommended adjunctive therapies: Small RCTs failed to show the superiority of adjunctive therapy with guanfacine (Level 1, negative) [1057,1058], bupropion SR [1005] (Level 2, negative), or zolpidem [1059] (Level 2, negative). While case series suggested that adjunctive topiramate $[1026,1027,1029]$ may be effective in treatment-resistant PTSD, a RCT failed to show superiority over placebo [1028] (Level 2, negative).

Treatments for specific PTSD-associated symptoms

Several agents have been used to target particular symptoms associated with PTSD. Prazosin has demonstrated significant efficacy for reducing trauma nightmares and improving sleep quality in patients with PTSD compared 
with placebo (Level 1) [1035,1062-1066]. Some openlabel data suggest that naltrexone may help reduce flashbacks (Level 3) [1067-1070], and fluphenazine may improve trauma re-experiencing symptoms (Level 3) [1037].

Cyproheptadine was not effective for nightmares or sleep problems in patients with PTSD and may actually exacerbate sleep disturbance (Level 2, negative) [1071].

\section{Not recommended}

In general, data do not currently support the use of divalproex [1014-1017] (Level 1, negative), alprazolam [1006], citalopram [974,986-988], olanzapine [1036-1038], tiagabine [1018] (all Level 2, negative), or clonazepam (Level 3, negative) [881,1007,1008].

\section{Maintenance pharmacological treatment}

Long-term therapy has been evaluated in relapseprevention and naturalistic follow-up studies. Relapseprevention studies are those in which responders to SSRI therapy are randomized to continued active treatment or placebo. A meta-analysis of three relapseprevention studies included 272 patients with PTSD, and found a highly significant reduction in relapse rates with continued SSRI treatment compared with placebo over approximately six months (odds ratio for relapse was 0.25) [497].

In RCT discontinuation studies, fluoxetine [1072,1073] and sertraline [1074] have demonstrated significantly lower relapse rates over six months in the range of $5-22 \%$ with active treatment compared to $16-50 \%$ with placebo [1072-1074]. However, in a small discontinuation RCT, tiagabine was not superior to placebo in preventing relapse [1075].

Open follow-up studies with paroxetine [1076] and sertraline [1077] have demonstrated sustained and continued improvement over six to 12 months of continued SSRI therapy.

\section{Biological and alternative therapies}

In general, these therapies may be useful for some patients; however, more data are needed.

Biological therapies: In RCTs, rTMS was effective as monotherapy or as an adjunct to SSRIs in patients with PTSD (Level 1) [1078-1080], and at least some improvements were maintained at two to three months after treatment $[1078,1079]$. Open prospective and retrospective data suggest that adjunctive electroconvulsive therapy may be helpful in patients with refractory PTSD (Level 3) [1081,1082].

Alternative therapies: In a RCT, acupuncture was more effective than a wait-list control and as effective as group CBT (Level 2) [1083]. Adjunctive use of symptom-oriented hypnotherapy [1059] or mantra repetition [1084] (both Level 2) improved PTSD symptoms in small trials; and in a small case series, patients with
PTSD benefited from transcendental meditation (Level 4) [1085].

\section{Summary}

The lifetime prevalence of PTSD is around 6-9\%; it is more frequent in women than in men, with an onset generally in the mid to late 20s. PTSD is associated with high rates of functional impairment, somatic complaints, suicide risk, and comorbid psychiatric disorders. A diagnosis of PTSD requires evidence of exposure to trauma, and is characterized by intrusive and dissociative symptoms.

Evidence does not support the wide spread use of early intervention with psychological strategies for the prevention of PTSD. Debriefing of all trauma victims is not recommended, rather, screening and treating appropriate individuals is preferred. In general, there is little evidence supporting the use of pharmacotherapy for the prevention of PTSD, with most studies suggesting no preventive benefits.

CBT is an effective first-line option for the treatment of PTSD. Effective approaches include TF-CBT, EMDR, PE, and stress management therapy. ICBT and VRE have also demonstrated efficacy. Benefits are maintained during long-term follow-up of up to one to 10 years after treatment. Research evaluating combined psychological and pharmacological treatments in PTSD is limited, and this requires further study.

Pharmacotherapeutic approaches should begin with one of the first-line options which include SSRIs such as fluoxetine, paroxetine, or sertraline, or the SNRI venlafaxine XR. If response to optimal doses is inadequate or the agent is not tolerated, therapy should be switched to another first- or second-line agent, or a second-line agent should be added. Patients with PTSD may make few gains during treatment, and it is important to preserve even small gains achieved with initial therapy. Therefore, augmentation with second- or third-line agents may be important early in treatment.

Patients who do not respond to multiple courses of therapy are considered to have treatment-refractory illness. In such patients it is important to reassess the diagnosis and consider comorbid medical and psychiatric conditions that may be affecting response to therapy. Third-line agents, adjunctive therapies, as well as biological and alternative therapies may be useful when patients fail to respond to an optimal treatment trial of first- and second-line therapies used alone and in combination.

\section{Special populations}

\section{Women during pregnancy and the postpartum period}

\section{Epidemiology}

Women have been found to be at higher risk for anxiety and related disorders than men [2]. Anxiety disorders during the perinatal period are increasingly gaining 
research attention. Although further investigation is needed, data from a large national survey suggest the overall prevalence of anxiety and related disorders is unchanged in women during pregnancy [1086]; however, other data have found an increased risk for individual disorders, such as GAD $[1087,1088]$. Similarly, some data suggest that anxiety disorders are also not more prevalent during the postpartum period [1086], but other studies suggest higher rates of OCD and GAD during this period $[1089,1090]$. In addition, PTSD can develop as a result of pregnancy complications that are experienced as traumatic [1091,1092].

Anxiety and related disorders during pregnancy or postpartum may have a negative impact on the pregnancy, the child, or the mother. While studies report that maternal anxiety disorders are associated with adverse pregnancy outcomes such as a shorter gestational age, premature delivery, or elective cesarean delivery [1093-1095], a meta-analysis found no relationship between anxiety symptoms per se and adverse perinatal outcomes [1096]. Anxiety symptoms during pregnancy have been associated with depressive symptoms, substance use, and anemia, as well as decreased use of prenatal vitamins [1093,1097-1099].

Parenting may also be affected by maternal anxiety and related disorders. Mothers with anxiety disorders have been found to be less promoting of psychological autonomy than those mothers without anxiety [1100]. Maternal anxiety has been found to be predictive of child cognitive development [1101], associated with behavioral/emotional problems in childhood [1101,1102], and maternal anxiety and related disorders have been found to be related to subsequent development of an anxiety disorder in the child [1103].

\section{Treatment issues}

Psychosocial treatments, with CBT specifically, have strong empirical support for the treatment of anxiety and related disorders $[63,70,71,1104]$, but evidence of their efficacy in perinatal women with anxiety disorders is lacking. Cohort studies have shown beneficial effects of group CBT in pregnant women with B-I-I phobia [1105], and individual CBT in women with OCD in the postnatal period [1106]. Arch et al. argued that although exposurebased CBT or behavioral therapy may have been avoided in the past because of concerns of potential harm, they likely can be viable, safe alternatives in pregnancy [1107]. The lack of data on the use of structured psychosocial interventions for anxiety and related disorders during the perinatal period is a significant gap in the literature.

It is important to consider the risks and benefits of pharmacotherapy during pregnancy and while breastfeeding during the postpartum period. Risks to the fetus and newborn should be weighed against that of the potential harm of untreated anxiety and related disorders, an area that is gaining increasingly more research attention. Treatment decisions should attempt to optimize outcomes for both mother and baby.

Detailed recommendations on the use of psychiatric medications during pregnancy and lactation are available from the American Congress of Obstetricians and Gynecologists (ACOG) Practice Bulletin [1108]. Although it is over five years old, risks associated with various psychotropic medications are summarized [1108]. The FDA pregnancy risk category system has been criticized as being insufficient [1109] and is currently under the process of revision. The Canadian Hospital for Sick Children Motherisk website (http://www.Motherisk.org) is also a useful resource.

Antidepressants: There appears to be little evidence of an association between maternal antidepressant use and increased risks of congenital malformations in general, and major congenital malformations in infants [1110-1113]. The exception is a statistically increased risk of cardiac defects with antidepressants, and with paroxetine specifically, although the clinical significance of this has been questioned [1108,1113-1117]. There have been reports of increased rates of spontaneous abortion following antidepressant use during pregnancy; in the most recent metaanalysis, this was not supported using data from studies with higher study quality but found by others who included all studies [1118-1120]. In terms of delivery outcomes, a recent meta-analysis found a statistically increased risk for preterm birth, lower gestational age, birth weight, and APGAR scores - but the effects were small, generally in the normal range, and of questionable clinical significance [1118]. However, data support an increased risk for poor neonatal adaptation syndrome (PNAS) [1121-1123], while findings of increased risk for persistent pulmonary hypertension in the antenatally exposed infant have not been consistent [1124-1127]. Systematic reviews suggest that overall prenatal exposure to antidepressants does not appear to be associated with changes in long-term neurocognitive or behavioral development in children [1128-1130] and that illness itself appears to play a role in negative outcomes (although this study examined the effects of maternal depression) [1131]. Two reports link prenatal antidepressant use to childhood autism spectrum disorders [1132,1133] and two others link bupropion exposure to childhood ADHD $[1134,1135]$. These studies have limitations and further research is required.

In terms of breastfeeding, potential risks of antidepressant use during lactation must be weighed against the recognized benefits for the infant. Antidepressants are excreted into breast milk and although data are limited, the majority are found in very low amounts with few isolated instances of adverse signs [1108]. If antidepressant treatment is indicated, sertraline or paroxetine is preferred [1136]. Long-term data on potential neurobehavioral 
effects are lacking. Clinicians can consult LactMed at http://toxnet.nlm.nih.gov/cgi-bin/sis/htmlgen?LACT for the latest information available.

Benzodiazepines: The data on benzodiazepines remain more limited. A recent meta-analysis did not find an increased risk of major malformations or cardiac defects following prenatal benzodiazepine exposure, but concluded the significant increase in risk of oral cleft remains based on data derived from case-control studies [1137], although another meta-analysis reported the absolute risk is small $(<1 \%)$ [1138]. A case-control study published in 2002 examining exposure to five benzodiazepines (including clonazepam) and not included in the above meta-analyses, with over 60,000 infants, did not find an association with various congenital malformations or oral clefts [1139]. Although there are a lack of meta-analytic data, neonatal withdrawal or toxicity syndrome has been described with antenatal benzodiazepine exposure and close monitoring of the infant has been recommended [1108]. The neurobehavioral effects on the child over the long-term due to antenatal exposure have been topics of debate and remain uncertain [1108]. Benzodiazepines are excreted into breast milk at low levels generally. A recent study with 124 mothers documented low levels of adverse effects (sedation in particular) and supported the initiation of breastfeeding [1140]. Caution may be advised regardless however in infants who poorly metabolize benzodiazepines [1108].

Atypical antipsychotics: Data on the use of antipsychotics during pregnancy continue to be limited [1141]. Thus far, there does not appear to be an increased risk for malformations although inconsistent data have been reported with some suggesting the data are inconclusive [1141-1143]. These drugs have been found to be associated with both increased and decreased birth weight as well as increased risk for preterm birth [1144-1149]. The second-generation antipsychotics can increase the risk of complications given the risk of metabolic syndrome, and thus diabetes, in the mother. Monitoring has been recommended [1150]. Both the FDA and Health Canada have issued safety alerts advising of the potential risk for abnormal muscle movements and withdrawal symptoms in infants exposed to antipsychotic medications during the $3^{\text {rd }}$ trimester of pregnancy [1151-1153]. Data on breastfeeding are more limited, but levels in breast milk have typically been shown to be low although adverse effects have been reported [1154].

\section{Summary}

The management of anxiety and related disorders in women who are pregnant or lactating requires careful consideration of both the potential risks of any treatment option as well as risks of an untreated anxiety disorder. Antidepressants are generally associated with low teratogenic risk and adverse delivery outcomes. Patients should be counseled about PNAS and its management. Less is known about the risk of benzodiazepine and atypical antipsychotic exposure during pregnancy as the data are more limited. Treatment must be individualized and decisions should be made with the most up-to-date information with the best course of action decided upon with the patient. Poorly or untreated psychiatric illness carries its own risks, both in the short- and long-term.

\section{Children and adolescents \\ Epidemiology}

Anxiety and related disorders were the most common psychiatric disorders noted in the National Comorbidity Survey-Adolescent supplement (NCS-A) (age 13-18 years), with a lifetime and 12 -month prevalence of $31.9 \%$ and $24.9 \%$, respectively [1155,1156]. Prevalence rates for individual anxiety and related disorders are shown in Table 31 [1155,1156].

Specific phobias are very common in children. However, although most adolescents reported at least one fear $(77 \%)$, lifetime prevalence rates are in the range of $10-35 \%[308,1156]$. A study including children as young as five years of age found lower rates of diagnosed specific phobias (1\%) [1157]. B-I-I and animal fears are the most common types reported in pediatric populations $[308,1157]$. The prevalence of OCD is only $0.25 \%$ in children [1158], but is $1-2 \%$ in adolescents, which is comparable to the rate seen in adults $[2,1159,1160]$.

In the adolescent population, anxiety and related disorders were found to have the earliest median age of onset (six years), compared to other psychiatric disorders (11-15 years) [1156]. Similarly, in the adult population, the median age of onset was earliest for anxiety and related disorders (11 years) compared to other psychiatric disorders (20-30 years) [2]. Separation anxiety disorder and the phobias (seven to 14 years) have much earlier median ages of onset compared to OCD, GAD, panic disorder, or PTSD (20-30 years) [1,2,1161].

Anxiety and related disorders can have a substantial long-term impact, putting children at elevated risk for

Table 31 Prevalence estimates of anxiety and related disorders among youths in the NCS-A (age 13-18 years)

\begin{tabular}{lcc}
\hline Anxiety and related disorder & \multicolumn{2}{c}{ Estimated prevalence (\%) } \\
\cline { 2 - 3 } & 12-month & Lifetime \\
\hline Any anxiety disorder & 24.9 & 31.9 \\
Separation anxiety disorder & 1.6 & 7.6 \\
Specific phobia & 15.8 & 19.3 \\
Social anxiety disorder & 8.2 & 9.1 \\
Posttraumatic stress disorder & 3.9 & 5.0 \\
Panic disorder & 1.9 & 2.3 \\
Generalized anxiety disorder & 1.1 & 2.2
\end{tabular}

Adapted from references $[1155,1156]$. NCS-A = National Comorbidity SurveyAdolescent supplement 
MDD, other anxiety disorders, and SUD in adulthood $[11,12]$. Anxiety and related disorders among younger patients are associated with high rates of comorbid psychiatric conditions [1162-1165], SUD [1166-1169], sleep problems [1170-1173], somatic symptoms [1174], and suicidality [1175], as well as problems with cognition/ attention [1164,1176,1177], academic performance $[1178,1179]$, and peer relationships [1180].

\section{Diagnostic issues}

Diagnostic evaluation of pediatric patients should be based on DSM-5 criteria, but use developmentally appropriate language, and consider collateral information from parents and teachers. Children may express anxiety through crying, tantrums, freezing, or clinging, as well as through play. The DSM-5 provides some modifications to adult criteria to assist in the diagnosis of anxiety and related disorders in children (Table 32) [26]. In particular, a separate subtype for patients $\leq 6$ years of age has been added to the criteria for PTSD to make it more developmentally sensitive to young children [26].

\section{Prevention strategies}

Psychoeducational programs for children and adolescents aimed at preventing the development of an anxiety or related disorder have shown small, but significant effects [1181]. Both universal (administered to all children within target population) [1182] and indicated prevention programs (administered to children demonstrating highly anxious symptoms) [1183,1184] demonstrate benefits, but indicated programs are associated with larger effect sizes than universal programs [1181].

Both psychological and pharmacological strategies have been assessed for the prevention of PTSD. An early psychological intervention with children involved in road traffic accidents failed to result in any significant benefits over a control group [1185].

In a RCT in burn victims, sertraline was more effective in preventing PTSD symptoms than placebo according to parent report but not child report [890]. Data do not support the use of propranolol in preventing PTSD [1186] or ASD [886] in pediatric injury patients.

\section{Treatment issues}

Psychological treatment Psychological therapies for children often need to be adapted to suit the chronological and developmental ages of young patients and to include parental involvement. Meta-analyses support the efficacy of CBT for the treatment of anxiety and related disorders in children and adolescents [1187-1191]. A meta-analysis of 24 clinical trials showed that almost $70 \%$ of youths who received CBT no longer met diagnostic criteria for their anxiety disorder compared to only $13 \%$ of wait-list controls [1189]. Meta-analyses and RCTs have confirmed the efficacy of CBT in children with SAD [1192-1197], panic disorder [1198], OCD [1199-1204], PTSD [946,1205-1211], school refusal [1212-1215], and separation anxiety disorder [1216].

Table 32 DSM-5 diagnostic criteria for anxiety and related disorders specific to children

\begin{tabular}{|c|c|}
\hline $\begin{array}{l}\text { Anxiety or related } \\
\text { disorder }\end{array}$ & DSM-5 diagnoses specific to children \\
\hline $\begin{array}{l}\text { Separation anxiety } \\
\text { disorder }\end{array}$ & $\begin{array}{l}\text { - Developmentally inappropriate and excessive fear or anxiety concerning separation from those to whom the individual is } \\
\text { attached, as evidenced by } \geq 3 \text { of the following: } \\
\text { o Distress when separation occurs, worry about loss or separation, reluctance to leave home, be alone, or go to sleep } \\
\text { because of fear of separation, nightmares involving separation, or complaints of physical symptoms (e.g., headaches, upset } \\
\text { stomach) when separation occurs } \\
\text { - Duration of at least } 4 \text { weeks } \\
\text { - Onset before } 18 \text { years of age } \\
\text { - The disturbance causes clinically significant distress or impairment in social, academic (occupational), or other important } \\
\text { areas of functioning }\end{array}$ \\
\hline Selective mutism & $\begin{array}{l}\text { - Consistent failure to speak in specific social situations in which there is an expectation for speaking (e.g., at school) } \\
\text { despite speaking in other situations }\end{array}$ \\
\hline $\begin{array}{l}\text { Anxiety or related } \\
\text { disorder }\end{array}$ & Changes to adult DSM-5 diagnostic criteria specific to children \\
\hline Specific phobia & $\begin{array}{l}\text { - The fear or anxiety may be expressed by crying, tantrums, freezing, or clinging } \\
\text { - Other specifiers: loud sounds or costumed characters }\end{array}$ \\
\hline SAD (social phobia) & $\begin{array}{l}\text { - The anxiety must occur in peer settings, not just during interactions with adults } \\
\text { - The fear or anxiety may be expressed by crying, tantrums, freezing, clinging, shrinking, or failure to speak in social } \\
\text { situations }\end{array}$ \\
\hline OCD, panic disorder & - No pediatric specific criteria \\
\hline PTSD & $\begin{array}{l}\text { - Qualifiers in children } \\
\circ \text { Intrusion symptoms: repetitive play may occur in which themes or aspects of the traumatic event(s) are expressed; } \\
\text { there may be frightening dreams without recognizable content; trauma-specific re-enactment may occur in play } \\
\text { - Specific subtype for children } \leq 6 \text { years of age }\end{array}$ \\
\hline GAD & - Less stringent criteria for symptoms than in adults \\
\hline
\end{tabular}


CBT has demonstrated efficacy in both group and individual formats $[1189,1190,1194,1217,1218]$, as well as in computer- or internet-based formats $[1219,1220]$.

One commonly used pediatric CBT protocol is the "Coping Cat" program [1221,1222], which has demonstrated efficacy in RCTs [1221,1223,1224] and in longterm follow-up studies [1222,1225]. In a RCT, Coping Cat CBT was as effective as pharmacotherapy with an SSRI, but less effective than combination therapy $[1223,1224]$.

Additional specific psychological approaches that have demonstrated efficacy in treating anxiety in children and adolescents include: attention bias modification (ABM) [1226], MBCT [1195], and social effectiveness therapy (SET) $[1227,1228]$ for SAD; ERP [1229,1230], family-based CBT [1231,1232], and meta-cognitive therapy [1229] for OCD; cognitive behavioral writing therapy (CBWT) [1233], spiritual-hypnosis assisted therapy (SHAT) [1234], emotion regulation therapy [1235], exposure therapy [1236], and EMDR [905,1237,1238] for PTSD; and exposure therapy for specific phobias [313].

Approaches that include parental or family involvement may have some additional benefit over strategies that include children only [1239-1245], especially when parents suffer from an anxiety or related disorder themselves [1246]. Parental training only has also demonstrated beneficial effects on children with an anxiety disorder [1247,1248].

The presence of comorbidities may have a negative impact on the efficacy of CBT in pediatric patients [1249]. However, integrated CBT protocols designed to target both conditions have demonstrated efficacy in youths with anxiety and related disorders and comorbid ADHD [1250], aggression [1251], or comorbid SUD [1252].

Long-term follow-up studies have shown sustained benefits of CBT over two to seven years posttreatment $[1218,1225,1228,1253,1254]$.

Pharmacological treatment Complete treatment recommendations for the management of anxiety and related disorders in youths are beyond the scope of these guidelines and the reader is referred to specific guidelines for the assessment and treatment of children and adolescents with anxiety disorders, such as those developed by the American Academy of Child and Adolescent Psychiatry (AACAP) [1255-1258].

For children and adolescents, psychological treatments are generally preferred over pharmacotherapy, or if warranted combination therapy may be an option. RCTs comparing combined pharmacological and psychological treatments in younger patients with anxiety have demonstrated efficacy equal or superior to either treatment alone $[1199,1223,1224,1259]$. In the pediatric population, safety concerns associated with antidepressants (see "Safety Issues") should be weighed against the potential benefits of therapy. Medication may be warranted in children and adolescents with severe impairment or those who are unlikely to respond to CBT due to cognitive or other issues. A "start low and go slow" approach is advised when using medications in this patient population.

The strength of evidence for pharmacotherapeutic agents in the treatment of pediatric patients is shown in Table 33. When pharmacotherapy is felt to be warranted, SSRIs are generally preferred for children and adolescents with anxiety and related disorders.

Antidepressants: SSRIs and TCAs have been well studied in pediatric patients with anxiety and related disorders (Table 33) [1260-1262], although these agents should be used with caution in youths as discussed in the section on safety issues below. Most of the data in pediatric patients are in those with OCD [1204,1261,1263] or SAD [1197].

There is good evidence for the efficacy of SSRIs in children and adolescents with OCD, including fluoxetine (Level 1) [1264-1269], citalopram (Level 2) [1264,1270], fluvoxamine (Level 2) [1271], paroxetine (Level 2) [1272], and sertraline (Level 2) [1273], as well as for the TCA clomipramine (Level 1) [1274-1276].

Similarly, there is good evidence for the efficacy of SSRIs in SAD, including fluoxetine (Level 1) [1227,1277], fluvoxamine (Level 2) [1278], paroxetine (Level 2) [1279], escitalopram (Level 3) [1280], and sertraline (Level 3) [1281], as well as for the SNRI venlafaxine XR (Level 2) [1282], and some evidence for mirtazapine (Level 3) [1283].

There is level 2 evidence for the efficacy of fluoxetine [1277] and fluvoxamine [1278] in separation anxiety disorder, and for fluoxetine [1277], fluvoxamine [1278], and sertraline [1284] in GAD. In school-refusing children and adolescents, a small case-series suggested benefit with citalopram (Level 4) [1285], and a RCT demonstrated that imipramine as an adjunct to CBT was more effective than CBT alone (Level 2) [1259].

In pediatric PTSD, sertraline alone [1286] or as an adjunct to CBT [946] was not more effective than placebo or CBT (both Level 2, negative) and cannot be recommended at this time.

Benzodiazepines: There are little data demonstrating the efficacy of benzodiazepines in children and adolescents with anxiety and related disorders (Table 33) [1287-1292]. In fact, the few RCTs have demonstrated no significant improvements in anxiety symptoms with alprazolam over placebo in overanxious or avoidant disorders (Level 2, negative) [1290] or school-refusal (Level 2, negative) [1291], or with clonazepam in separation anxiety disorder (Level 2, negative) [1292]. Benzodiazepines have limited utility in youths, although they may be useful for short-term therapy in specific situations where there is a need to achieve rapid reduction in severe anxiety symptoms to allow exposure-related psychotherapy (e.g., panic disorder, school refusal behavior). 
Table 33 Strength of evidence of treatments for anxiety and related disorders in children and adolescents

\begin{tabular}{|c|c|c|}
\hline Disorder & Antidepressants & Benzodiazepines and other treatments \\
\hline \multirow[t]{6}{*}{ OCD } & Fluoxetine (Level 1) [1264-1269] & Antipsychotics \\
\hline & Clomipramine (Level 1) [1274-1276] & Adjunctive aripiprazole (Level 3) [1293] \\
\hline & Citalopram (Level 2) $[1264,1270]$ & Other \\
\hline & Fluvoxamine (Level 2) [1271] & Riluzole (Level 4) [1294] \\
\hline & Paroxetine (Level 2) [1272] & \\
\hline & Sertraline (Level 2) [1273] & \\
\hline \multirow[t]{3}{*}{ Panic disorder } & & Anxiolytics \\
\hline & & Clonazepam (Level 4) [1287,1288] \\
\hline & & Alprazolam (Level 4) [1289] \\
\hline \multirow[t]{7}{*}{ SAD } & Fluoxetine (Level 1) $[1227,1277]$ & Anxiolytics \\
\hline & Fluvoxamine (Level 2) [1278] & Alprazolam (Level 2, -ve) [1290] \\
\hline & Paroxetine (Level 2) [1279] & \\
\hline & Venlafaxine XR (Level 2) [1282] & \\
\hline & Escitalopram (Level 3) [1280] & \\
\hline & Sertraline (Level 3) [1281] & \\
\hline & Mirtazapine (Level 3) [1283] & \\
\hline \multirow[t]{2}{*}{ Separation anxiety disorder } & Fluoxetine (Level 2) [1277] & Anxiolytics \\
\hline & Fluvoxamine (Level 2) [1278] & Clonazepam (Level 2, -ve) [1292] \\
\hline \multirow[t]{3}{*}{ GAD } & Fluoxetine (Level 2) [1277] & Anxiolytics \\
\hline & Fluvoxamine (Level 2) [1278] & Alprazolam (Level 2, -ve) [1290] \\
\hline & Sertraline (Level 2) [1284] & \\
\hline \multirow[t]{2}{*}{ School-refusal } & Citalopram (Level 4) [1285] & Anxiolytics \\
\hline & Adjunctive imipramine (Level 2) [1259] & Alprazolam (Level 2, -ve) [1291] \\
\hline \multirow[t]{2}{*}{ PTSD } & Sertraline (Level 2, -ve) [1286] & \\
\hline & Adjunctive sertraline (Level 2, -ve) [946] & \\
\hline
\end{tabular}

$\mathrm{XR}=$ extended release; $(-\mathrm{ve})=$ negative.

Other treatments: In open trials in pediatric patients with treatment-resistant OCD, the atypical antipsychotic aripiprazole (Level 3) [1293] and the glutamate antagonist riluzole (Level 4) [1294] have demonstrated some efficacy.

Combination psychological and pharmacological therapies The combination of sertraline and CBT was significantly superior to both monotherapies in a large RCT in pediatric patients with separation anxiety disorder, GAD, or SAD [1223]. In pediatric patients with OCD, the addition of CBT in those with a partial response to SSRIs resulted in significantly greater response rates compared with the SSRI alone [1199], while the addition of d-cycloserine to CBT was not superior to placebo [1295].

Alternative therapies There is currently little evidence supporting the efficacy of exercise in reducing anxiety symptoms in pediatric populations [1296], although some open data suggest it may have a small beneficial effect in pediatric PTSD [1297,1298].

Safety issues An important consideration when using antidepressant medications in children and adolescents is the potential for an increased risk of suicidality. Clinicians should be aware of the potential activating side effects of SSRIs (insomnia, agitation, tremor, and anxiety), especially in young children [1299-1301]. Regulatory bodies in many countries have issued black-box warnings about suicidal ideation/suicide attempts with the use of antidepressants in patients younger than 19 years. However, in a comprehensive analysis, the pooled absolute risk difference for suicidal thinking or behavior between SSRI- and placebotreated youth with anxiety and related disorders was nonsignificant (0.5-0.7\%), and lower than the risk for youth treated for MDD (0.9\%) [1302]. Anxiety and related disorders also increase the risk of suicidality - nearly eight times for suicidal ideation and six times for suicide attempts compared with not having an anxiety disorder [1175]. Therefore, risks and benefits of treatment should be discussed with both children and their parents.

The most common antidepressant adverse events are generally activation and vomiting in children, and somnolence in adolescents [1303]. More conservative dosing strategies may be needed especially in younger children or those with low body weight [1301].

\section{Summary}

The management of anxiety and related disorders in children and adolescents can be challenging. Diagnostic evaluation of pediatric patients should use developmentally appropriate language and consider collateral information 
from parents and teachers. Children may express anxiety through crying, tantrums, freezing, or clinging, as well as through play. For children and adolescents, psychological therapies are generally preferred over pharmacotherapy, or if warranted combination therapy may be an option. Psychological therapies often need to be adapted to suit the chronological and developmental ages of young patients and to include parental involvement. When pharmacotherapy is warranted, SSRIs are generally preferred, although antidepressants should be used with caution in pediatric patients.

\section{Elderly}

\section{Epidemiology}

The lifetime and 12-month prevalence of any anxiety or related disorder among those age 65 or older is estimated to be $13.6 \%$ and $7.0 \%$, respectively, compared with $27.8 \%$ and $17.8 \%$ in the overall adult population [1304]. Including subthreshold anxiety increases the 12-month prevalence from about $6 \%$ to over $26 \%$ in older adults [1305]. The prevalence rates of anxiety and related disorders have generally been shown to decline with age, and as in younger age groups, the prevalence is higher in women than in men $[509,1304,1306,1307]$. The decline in prevalence may be related to age biases in the assessment of anxiety and the masking effect of other risk factors that increase with aging [1308]. Under-diagnosis is common, with one study finding that only $34 \%$ of older patients with GAD had previously had anxiety symptoms documented [1309].

Among older adults ( $\geq 55$ years) with mood or anxiety and related disorders, $60-70 \%$ do not use mental health care services $[1310,1311]$, although use is higher among those with comorbid disorders [1312]. Older adults with anxiety and related disorders have higher rates of sleep disturbances [1313-1315] and greater impairment in cognitive functioning [1316-1319] compared to those without anxiety disorder. In addition, anxiety negatively impacts physical functioning and mobility [1320,1321], and health related QoL [1321,1322].

Comorbidities Depression is among the most common comorbid disorders among older adults with anxiety and related disorders [1323-1325], and is associated with poorer outcomes of both disorders [1326]. Approximately $80 \%$ of adults $\geq 65$ years of age have at least one chronic medical condition, and this may be even higher among those with anxiety disorders [1327]. Older patients with anxiety and related disorders report higher rates of diabetes, gastrointestinal conditions, and dementia [1325,1327,1328].

Chronic urinary incontinence, hearing impairment, hypertension, respiratory disease, and poor sleep were associated with elevated rates of anxiety symptoms or disorders [1315,1329]. Comorbid anxiety in patients with medical illnesses, particularly cardiovascular disease, has been associated with an increased risk of mortality
[1330,1331]. Furthermore, the relationship between anxiety and related disorders in the elderly and cognitive impairment remains largely neglected [1332].

\section{Diagnostic issues}

The recognition and accurate diagnosis of anxiety and related disorders in older patients can be challenging [1333]. Modifications to the DSM-5 diagnostic criteria may assist clinicians in more accurately recognizing and diagnosing anxiety and related disorders in the elderly [1333].

Older patients with anxiety often present differently than younger patients $[1327,1334]$. Avoidance and excessive anxiety may be difficult to detect in older patients [1333]. Older adults may describe symptoms differently; for example, they may discuss concerns rather than worries $[1327,1333]$. They are less likely to attribute symptoms to anxiety and related disorders, but rather may attribute them to physical illness and they may have difficulty remembering symptoms [1327,1335]. Obtaining information from collateral sources may be useful. Assessing impact on work or social functioning may also be complicated by changes in responsibilities associated with aging (e.g., retirement) [1333]. It may be helpful to ask about activities relevant to older adults, such as visiting grandchildren. Similarly, avoidance may be harder to detect because of limitations in physical mobility or visual problems, leading to a decline in activities outside the home [1336].

Chronic medical illness or the use of medications can also complicate the diagnosis of anxiety and related disorders [1333]. Determining which came first, the physical illness or the anxiety symptoms can be helpful. However, when a medical illness is chronic, this precludes the likelihood that the anxiety would resolve when the medical condition resolves.

Late-onset anxiety and related disorders are relatively unusual [2], therefore older patients with new onset anxiety should be investigated for potential causative factors (e.g., physical illness, medication side effects).

\section{Psychological treatment}

Relaxation training, CBT, supportive therapy, and CT have support for the treatment of anxiety symptoms and disorders in older patients [1337]. Meta-analyses suggest the efficacy of psychological treatment is similar to that of pharmacotherapy for the treatment of anxiety and related disorders in older patients [1338,1339].

In meta-analyses, CBT was an effective option in reducing anxiety symptoms among older patients compared to wait-list or active controls [1340-1342]. Some data suggest that CBT may be less effective for anxiety and related disorders in older patients than in working-age adults $[1337,1342]$. Older patients may benefit from the inclusion of learning- and memory-aids with standard CBT $[1343,1344]$. In RCTs in older patients, CBT demonstrated 
efficacy for the treatment of GAD [1337,1343,1345-1347] and panic disorder $[1348,1349]$. Exposure therapy, with or without CBT, demonstrated efficacy in case-controlled studies in patients with PTSD [1350] or specific phobias [1351]. CBT may also be effectively delivered via telephone, although improvements may not be long lasting [1352].

In a case-control study, regular physical exercise reduced the risk of developing anxiety disorders among older adults [1353].

\section{Pharmacological treatment}

Data suggest that pharmacotherapy including antidepressants or anticonvulsants is likely as effective in older adults as it is in younger patients [575]. Most of the studies in older patients include those $\geq 60$ or 65 years and have been conducted in patients with GAD or panic disorder.

The most robust data in elderly patients with GAD are from a large RCT $(n=273)$, which demonstrated significant improvements and good tolerability with pregabalin compared with placebo [1354]. Pregabalin was also effective as adjunctive therapy in an open trial in older patients with comorbid GAD and depression [1355].

Pooled analyses of subsets of older patients from multiple RCTs demonstrate that duloxetine [1356] and venlafaxine [575] were effective for the treatment of GAD. Citalopram was effective in an eight-week RCT [1357] and in an open study over six months of treatment [1358]. Some data suggest that escitalopram may be useful in older patients with GAD [550,1359]; although in one RCT, response rates were not significantly different than placebo in the intention-to-treat analysis [550]. Sertraline was more effective than CBT [1349], particularly at a one-year follow-up assessment [1360], and was as effective as buspirone [561] in older adults with GAD.

In older patients with panic disorder, paroxetine was as effective as CBT and more effective than a wait-list control, and results were sustained at a six-month follow-up [1348]. Escitalopram [1361] and citalopram [1361] were equally effective in a small, open trial. A small, open trial also showed fluvoxamine to be effective in older patients with GAD, panic disorder, or OCD [1362]. Data in patients with MDD, suggest that mirtazapine may have beneficial anxiolytic effects in the elderly $[1363,1364]$.

Data show that $45-60 \%$ of older patients ( $>55$ years) with anxiety and related disorders are prescribed a benzodiazepine, which is higher than the rate of antidepressant use [1365-1367]. The very high use of these agents is a cause for concern since they are not a preferred longterm treatment strategy and elderly patients may be more sensitive to their negative effects $[1365,1366]$.

Safety issues The elderly maybe more susceptible to adverse drug events and drug-drug interactions (DDIs) due to gradual age-related physiologic changes that affect the pharmacokinetic and pharmacodynamic properties of many medications $[1368,1369]$. Factors which may alter drug metabolism and plasma concentrations among elderly patients include frailty, reduced homoeostatic mechanisms, and psychosocial issues [1368]. Age-related changes in body composition can result in increases or decreases of drug volume distribution, and hepatic or renal dysfunction can impair drug metabolism and drug clearance $[1369,1370]$. All of these changes are highly variable in elderly patients, further complicating use of medications in this population [1368,1369]. A review of the literature found that almost half of available antidepressants are associated with age-related clearance changes and identified at least 45 medications that could interact with specific antidepressants [1371].

DDIs may be more common in older adults because of the greater number of concomitant medication they may be taking to treat multiple comorbid conditions. In one study of US community-dwelling older adults, almost $30 \%$ used at least five prescription medications, $80 \%$ used at least one prescription medication, and almost half used over-the-counter and dietary supplements [1372].

Psychotropic medications have been associated with an increased risk of fractures [1369,1373,1374]. In a metaanalysis, the RR of fractures was 1.34 for benzodiazepines, 1.60 for antidepressants, 1.54 for anticonvulsants, and 1.59 for antipsychotics [1373]. In a prospective cohort study (The Rotterdam Study) of subjects over 55 years of age, the risk of non-vertebral fractures was 2.35 for current SSRI use versus non-use [1375]. The increased risk for hip fracture associated with benzodiazepines was further increased with increasing dose and the use of concomitant interacting drugs $[1369,1374]$. There does not appear to be any difference between atypical antipsychotic agents in the increased risk of falls or fractures [1376].

An increased mortality risk has been associated with the use antipsychotics in older patients with dementia [1377-1379], which appears to be greater with conventional compared to atypical antipsychotics [1378-1380].

Antidepressants are frequently used to treat symptoms of anxiety in older adults who suffer from comorbid medical conditions such as heart disease. In a meta-analysis of SSRIs versus placebo or no antidepressant therapy in patients with coronary heart disease (CHD) and depression, SSRIs were associated with lower rates of all-cause mortality and readmissions for $\mathrm{CHD}$, indicating that treatment may improve CHD prognosis [1381]. Clinicians should weigh the risks associated with antidepressants against the potential benefits when making prescribing decisions.

\section{Summary}

While onset of anxiety and related disorders in late-life is uncommon, they do persist into older age and can have substantial impact on QoL and functionality. Older 
patients can present differently compared to younger patients, and diagnosis can be complicated by communication barriers, changes in role functioning, memory difficulties, and comorbid medical conditions.

Few treatment studies are conducted in older patients; however, data suggest that psychological treatment and pharmacotherapy appear to be similarly effective in older patients. Using pharmacotherapy in elderly patients can be challenging, and should consider patient factors such as body mass, hepatic and renal function, comorbid conditions, and use of concomitant medications.

\section{Anxiety with comorbid conditions Overview}

Anxiety and related disorders often present together with other psychiatric or medical conditions $[3,16,43$, 1382,1383]. About $60-80 \%$ of patients with an anxiety disorder have at least one other comorbid psychiatric condition, which most commonly include another anxiety or related disorder, MDD, bipolar disorder, ADHD, and SUD [3]. The presence of comorbid disorders has a negative impact on most aspects of care. Patients with psychiatric comorbidities have more severe symptoms $[46,1384]$, poorer treatment outcomes for both disorders [47,1385-1387], greater functional impairment $[46,871,1384]$, poorer QoL $[1388,1389]$, and an increased risk of suicide [652].

Medical conditions and pain disorders are also common comorbidities in patients with anxiety and related disorders. Medical conditions frequently reported in patients with anxiety and related disorders include cardiovascular disease, gastrointestinal disease, arthritis, respiratory disease, thyroid disease, migraine, and allergic conditions $[16,52]$. Patients with both anxiety disorders and medical conditions experience elevated disability, including more psychiatric comorbidity and depressive symptoms, as well as poorer interpersonal and physical functioning [52,142]. Patients with chronically painful conditions such as arthritis, back pain, or migraine are at a two- to four-fold higher risk of having an anxiety or related disorder, particularly panic disorder or PTSD [1390].

The high probability of comorbid disorders should be considered when diagnosing and treating patients with anxiety and related disorders. In patients with comorbid psychiatric conditions, such as another anxiety disorder or mood disorder, consider therapies that are effective for both disorders [32]. Benzodiazepines should be prescribed with additional caution in patients with comorbid SUDs. In patients with comorbid medical conditions, the clinician must weigh the benefits and risks of medication for the anxiety or related disorder, but should also consider the impact of untreated anxiety [32].

\section{Major depressive disorder (MDD)}

\section{$Q$. What is the prevalence and impact of comorbid MDD} and anxiety/related disorders?

MDD is very common in patients with anxiety, being reported in $20-36 \%$ of patients [121,310,360,1382]; and conversely, about $60 \%$ of patients with MDD will have a comorbid anxiety or related disorder [44]. In patients with anxiety, comorbid depression has been associated with more severe symptoms [46,1384], lower likelihood of remission [47], greater functional impairment $[46,871,1384]$, an increased risk of suicide [652], and a greater risk of having another comorbid anxiety disorder [360]. Similarly, in patients with MDD, comorbid anxiety and related disorders were associated with poorer treatment outcomes including higher recurrence rates [1385-1387], poorer QoL [1391], and an increased risk of suicide [24,1387,1392,1393].

\section{Q. What pharmacological treatment may be useful for} patients with an anxiety/related disorder and comorbid MDD? Guidelines generally recommend antidepressants (most commonly SSRIs and SNRIs) as first-line treatments in patients with both anxiety and depressive symptoms $[32,1394]$. SSRIs and SNRIs in patients with anxiety and related disorders, including panic disorder, GAD, OCD, or PTSD, with comorbid MDD have been shown to be effective in improving both disorders [224,723,1359,1395]. Among the atypical antipsychotics, quetiapine has been found to have efficacy as monotherapy in both MDD [1396] and GAD [1397], as well as MDD with anxiety [1398], while case series suggest that aripiprazole augmentation of antidepressants [496], and risperidone monotherapy [267] may also reduce comorbid depressive and anxiety symptoms.

\section{Bipolar disorder or psychoses \\ Q. What is the prevalence and impact of comorbid bipolar disorder or psychoses with anxiety/related disorders?}

Among patients with anxiety and related disorders, almost $14 \%$ also met criteria for bipolar I or II disorder [121]. However, among patients with bipolar disorder the rates of comorbid anxiety disorders are very high compared to the general population, and the DSM-5 notes anxiety disorders as the most common comorbidities in patients with bipolar disorder [26]. In epidemiological surveys, the lifetime comorbidity rates for any anxiety or related disorder among patients with bipolar disorder was 52\% in Canada [43] and $60-75 \%$ in the US $[1389,1399]$. In a clinic population, the rate of anxiety and related disorders was $22 \%$ in patients with bipolar disorder, compared to $17 \%$ in patients with schizophrenia, and $30 \%$ in those with schizoaffective disorder [1400]. A meta-analysis of prevalence studies found that the rates of various anxiety disorders in patients with schizophrenia and related psychotic disorders ranged from 10-15\% [1401]. 
Comorbid anxiety and related disorders in patients with bipolar disorder were associated with a greater risk of MDD and drug use disorders, a poorer bipolar course, lower QoL, and lower psychosocial functioning $[1388,1389]$. Data are conflicting on the impact of anxiety and related disorders on suicidal tendencies in patients with bipolar disorder, with some analyses finding an increased risk [1389,1402], but not all [1403]. Similar findings have been reported in patients with schizophrenia, where comorbid anxiety and related disorders have been associated with more past SUDs, lower social adjustment and overall QoL, and greater suicidality [1404,1405].

\section{Q. What pharmacological treatment may be useful for patients with an anxiety/related disorder and comorbid bipolar disorder or psychoses?}

The management of patients with anxiety and related disorders and comorbid bipolar disorder, schizophrenia, or other psychosis should consider therapies that are effective for both disorders [32]. Atypical antipsychotics are recommended treatments for bipolar disorder and schizophrenia $[111,1406]$, while the long-term use of antidepressants may destabilize patients with bipolar I disorder [111,1394].

Data in patients with a diagnosed anxiety or related disorder and comorbid bipolar disorder or psychosis are limited. In a RCT, risperidone monotherapy was shown to be no more effective than placebo for patients with bipolar and comorbid panic disorder or GAD [1407]. However, in a single-blind trial, olanzapine or lamotrigine when added to lithium demonstrated improvements in anxiety disorder symptoms in patients with remitted bipolar disorder [1408]; and in an open trial, switching to aripiprazole significantly improved social anxiety and psychosis in patients with SAD and schizophrenia [379]. In addition, atypical antipsychotics have demonstrated efficacy in RCTs in patients with anxiety and related disorders (see specific disorder sections for evidence), and data show that these agents can significantly reduce anxiety symptoms in patients with bipolar disorder [1409-1413]. Taken together, these data suggest these agents may be useful in comorbid patients.

Anticonvulsants have also demonstrated efficacy in the treatment of some anxiety and related disorders (see specific disorder sections for evidence) and are often used for the treatment of bipolar disorder [111]. In patients with bipolar disorder, adjunctive valproate and gabapentin have demonstrated efficacy for the treatment of panic disorder [281,1414] and resulted in reductions in anxiety symptoms $[1415,1416]$.

\section{ADHD}

Q. What is the prevalence of comorbid ADHD and anxiety/ related disorders?

It is estimated that the lifetime rate of ADHD in children is $6-9 \%$, with $70 \%$ persistence into adolescence and
$50-60 \%$ into adulthood $[45,1417,1418]$. In a communitybased survey, the estimated prevalence of current selfreported adult ADHD was 4.4\% [45]. While ADHD has long been known to persist into adulthood [1419,1420], it has only recently become the focus of widespread clinical attention [1421-1423].

Of adults identified with ADHD in the National Comorbidity Survey-Replication (NCS-R), only one in 10 had received treatment within the previous year [45]. Of these individuals, it is estimated that approximately $47 \%$ meet criteria for an anxiety or related disorder within 12 months of assessment, with the most common being SAD (29.3\%), followed by specific phobia (22.7\%), PTSD (11.9\%), panic disorder (8.9\%), and GAD (8.0\%) [45]. Patients with an anxiety or related disorder were reportedly four times more likely to meet criteria for ADHD than the general population [45]. Similar results were found in a Canadian survey of patients in an anxiety disorders clinic, where the rate of adult ADHD was $28 \%$ [378].

\section{Q. What factors should be considered when treating patients} with an anxiety/related disorder and comorbid ADHD?

When managing a patient with ADHD, it may be important to differentiate ADHD with anxious symptoms from comorbid ADHD and anxiety/related disorders. This can be challenging, as anxiety symptoms are frequently related to a sense of being overwhelmed or to compensatory skills in patients with ADHD. Stimulants may play a larger role in managing $\mathrm{ADHD}$ in patients with anxiety symptoms [1424,1425]; however, in an open trial, atomoxetine improved ADHD and comorbid symptoms of depression and anxiety [1426].

Treatment of patients with comorbid ADHD and an anxiety or related disorder may be more complicated. Generally, in patients with comorbid anxiety disorders and ADHD the diagnostic and treatment priority should be determined by the relative severity of symptoms and risks of each disorder [1427]. There are limited data on the role of stimulants in patients with ADHD and an anxiety disorder. In a RCT, atomoxetine significantly improved ADHD and SAD symptoms compared with placebo [487]. In separate open trials, adjunctive atomoxetine [1428] and adjunctive extended release mixed amphetamine salts [1429] significantly improved anxiety symptoms in patients with ADHD and GAD refractory to antidepressants alone.

\section{Medical comorbidities \\ Q. What is the prevalence and impact of comorbid medical conditions and anxiety/related disorders?}

Medical conditions are also common comorbidities that must be considered when prescribing medication for patients with anxiety and related disorders. Medical conditions are reported in over $60 \%$ of patients with anxiety 
and related disorders including cardiovascular diseases, gastrointestinal diseases, arthritis, respiratory diseases such as asthma, thyroid disease, migraine headaches, back pain, and allergic conditions [16,52,1430-1432]. Comorbidities are particularly common among patients with GAD, panic disorder, and PTSD [16,140,515, 517,1390,1433].

Patients with anxiety and related disorders and medical conditions experience more psychiatric comorbidity, depressive symptoms, and more severe anxiety disorder symptoms, as well as poorer interpersonal and physical functioning [52,140,142,515].

\section{Q. What factors should be considered when treating patients with an anxiety/related disorder and comorbid chronic pain?}

Chronically painful conditions (i.e., arthritis, back pain, and migraine) are commonly associated with anxiety $[515,1390,1430,1434]$. Patients with anxiety and related disorders are twice as likely to have painful physical symptoms compared to of those without, $45-60 \%$ versus $28 \%$ [515,1433]. About $60-70 \%$ of patients with anxiety disorders report migraine headaches [140,141].

For the management of anxiety and related disorders in patients with pain it may be helpful to consider treatments that have demonstrated efficacy in both anxiety disorders as well as pain. While there are few data available, duloxetine has demonstrated efficacy for both GAD and pain symptoms in RCTs [1435-1437]. TCAs, and to a lesser extent SSRIs, have been shown to reduce headache attacks in patients with migraine [1438], and provide moderate relief of neuropathic pain [1439].

Q. What factors should be considered when treating patients with an anxiety/related disorder and comorbid cardiovascular disease?

Although panic attacks can sometimes be mistaken for cardiovascular symptoms, it is important to be aware that patients with anxiety and related disorders do have a twoto three-times greater risk of cardiovascular disease compared to the general population $[1431,1432]$. In addition, anxiety disorders have been associated with increased risk of cardiovascular hospitalization rates and mortality risk [1440-1442]. In patients with cardiovascular or cerebrovascular comorbidity, it is important to consider the impact of treatments used for anxiety on heart rate, blood pressure, and lipid measures [1443-1445].

Q. What factors should be considered when treating patients with an anxiety/related disorder and comorbid diabetes and metabolic syndrome?

Patients with anxiety symptoms have an elevated risk of type 2 diabetes [1446]. While glycemic measures do not appear to be affected by anxiety symptoms [1447], some treatments, particularly some atypical antipsychotics, alter glucose parameters, lipid levels, and cause weight gain [109-116,1443]. Some antidepressants, including amitriptyline, mirtazapine, and paroxetine have also been associated with weight gain [1448].

\section{Canadian Anxiety Guidelines Initiative Group} Additional authors

Martin M. Antony ${ }^{1}$, Stéphane Bouchard ${ }^{2}$, Alain Brunet ${ }^{3}$, Martine Flament ${ }^{4}$, Sophie Grigoriadis ${ }^{5}$, Sandra Mendlowitz $^{6}$, Kieron O'Connor ${ }^{7}$, Kiran Rabheru ${ }^{4}$, Peggy M.A. Richter $^{5}$, Melisa Robichaud ${ }^{8}$, John R. Walker ${ }^{9}$

${ }^{1}$ Department of Psychology, Ryerson University, Toronto, M5B 2K3, Canada; ${ }^{2}$ Department of Psychoeducation and Psychology, University of Québec in Outaouais, Gatineau, J9A 1L8, Canada; ${ }^{3}$ Department of Psychiatry, McGill University, Montreal, H3A 1A1, Canada; ${ }^{4}$ Department of Psychiatry, University of Ottawa, Ottawa, K1Z 7K4, Canada; ${ }^{5}$ Department of Psychiatry, University of Toronto, Toronto, M5S 1A1, Canada; ${ }^{6}$ Department of Child Psychiatry, University of Toronto, Toronto, M5S 1A1, Canada; ${ }^{7}$ Department of Psychiatry, University of Montreal, Montreal, H3C 3J7, Canada; ${ }^{8}$ Departments of Psychiatry and Psychology, University of British Columbia, Vancouver, V6T 2A1, Canada; ${ }^{9}$ Department of Clinical Health Psychology, University of Manitoba, Winnipeg, R3E 3N4, Canada

Email: Martin M. Antony - mantony@psych.ryerson.ca; Stéphane Bouchard - stephane.bouchard@uqo.ca; Alain Brunet - alain.brunet@mcgill.ca; Martine Flament - martine.flament@theroyal.ca; Sophie Grigoriadis - sophie.grigoriaidis@sunnybrook.ca; Sandra Mendlowitz - sandra. mendlowitz@sickkids.on.ca; Kieron O'Connor - kieron. oconnor@umontreal.ca; Kiran Rabheru - kiranrabheru@hotmail.com; Peggy M.A. Richter - peggy.richter@sunnybrook.ca; Melisa Robichaud - robichau@mail.ubc.ca; John R.Walker - jwalker@cc.umanitoba.ca

\section{Additional contributors to the comorbidity section}

Gordon Asmundson ${ }^{10}$, Larry J. Klassen ${ }^{11}$, Raymond W. Lam $^{12}$, Roger S. McIntyre ${ }^{13}$, Isaac Szpindel ${ }^{14}$

${ }^{10}$ Department of Psychology, University of Regina, Regina, S4S 0A2, Canada; ${ }^{11}$ Department of Psychiatry, Faculty of Medicine, University of Manitoba, Winnipeg, R3T 2N2, Canada; ${ }^{12}$ Department of Psychiatry, University of British Columbia, Vancouver, V6T 2A1, Canada; ${ }^{13}$ Departments of Psychiatry and Pharmacology, University of Toronto, Toronto, M5S 1A1, Canada; ${ }^{14}$ Attention and Learning Related Disorders, START Clinic, Toronto, M4W 2N4, Canada

Email: Gordon Asmundson - gordon.asmundson@uregina.ca; Larry J. Klassen - larryjklassen@hotmail.com; Raymond W. Lam - r.lam@ubc.ca; Roger S. McIntyre roger.mcintyre@uhn.ca; Isaac Szpindel - iszpindel@startclinic.ca 


\section{Additional material}

Additional file 1: Suggested dosing ranges Dosing ranges of various psychiatric medications

\section{List of abbreviations used}

AACAP: American Academy of Child and Adolescent Psychiatry; ABM: attention bias modification; ACOG: American Congress of Obstetricians and Gynecologists; ADHD: attention-deficit/hyperactivity disorder; APA: American Psychiatric Association; ASD: acute stress disorder; B-I-l: blood-injection-injury; BPD: borderline personality disorder; CBT: cognitive behavioral therapy; CBWT: cognitive behavioral writing therapy; CCHS: Canadian Community Health Survey; CHD: coronary heart disease; CPT: cognitive processing therapy; CR: controlled release; DBT: dialectical behavioral therapy; DDI: drugdrug interactions; DIRT: danger ideation reduction therapy; DSM-5: Diagnostic and Statistical Manual of Mental Disorders, $5^{\text {th }}$ Edition; EMDR: eye movement desensitization and reprocessing; ERP: exposure with response prevention; FDA: Food and Drug Administration; GAD: generalized anxiety disorder; HARS: Hamilton Anxiety Rating Scale; HDL: high-density lipoprotein; ICBT: internet-based CBT; IPT: interpersonal therapy; IV: intravenous; LDL: lowdensity lipoprotein; MAOI: monoamine oxidase inhibitor; MBCT: mindfulnessbased cognitive therapy; MBT: mindfulness-based therapy; MDD: major depressive disorder; Mini-SPIN: Mini-Social Phobia Inventory; MRI: magnetic resonance imaging; N/A: not available; NaSSA: noradrenergic and specific serotonergic antidepressant; NCS-A: National Comorbidity Survey Adolescent supplement; NCS-R: National Comorbidity Survey - Replication; NMDA: N-methyl-D-aspartate; NNT: number needed to treat; NPPO-REAC: neuro psycho physical optimization-radio electric asymmetric conveyor; NSAID: nonsteroidal anti-inflammatory drug; OCD: obsessive-compulsive disorder; ODT: orally disintegrating tablet; PNAS: poor neonatal adaptation syndrome; PTSD: posttraumatic stress disorder; QoL: quality of life; RCT: randomized controlled trial; REAC: radioelectric asymmetric conveyor; RIMA: reversible inhibitors of monoamine oxidase A; RR: relative risk; rTMS: repetitive transcranial magnetic stimulation; SAD: social anxiety disorder; SET: social effectiveness therapy; SHAT: spiritual-hypnosis assisted therapy; SNRI: serotonin-norepinephrine reuptake inhibitor; SR: sustained release; SSRI: selective serotonin reuptake inhibitor; SUD: substance use disorder; TC: total cholesterol; TCA: tricyclic antidepressant; TF-CBT: trauma-focused-CBT; TG: triglycerides; VLDL: very-low-density lipoprotein; VRE: virtual reality exposure; XL: extended release; XR: extended release; Y-BOCS: Yale-Brown Obsessive Compulsive Scale.

\section{Competing interests}

Unrestricted educational grants for the development of these guidelines were provided by Astra Zeneca Canada, Eli-Lilly Canada, Janssen Inc., Lundbeck Canada, Pfizer Canada, Purdue Canada, Servier Canada Inc., Shire Canada, and Valeant Canada. None of the members received payment for participating in the development of these guidelines.

The following authors do not have any competing interests to declare: $A B, G A, J R W, K O, M M A, M F, L K, M R, S M$.

Advisory board/speaker's bureau: Astra Zeneca (KK, KR, MK, P. Bleau, P. Blier, RWL, RSM), Biovail (RWL), Boehringer Ingelheim (MK), BMS (KK, KR, MK, P. Bleau, P. Blier, PC, RWL, RSM), Canadian Institutes of Health Research (CIHR) (RWL), Canadian Network for Mood and Anxiety Treatments (CANMAT) (RWL), Canadian Psychiatric Association (CPA) Foundation (RWL) Eli Lilly Canada (MK, P. Bleau, P. Blier, PC, SG, RWL, RSM, IS), France Foundation (RSM), GlaxoSmithKline (MK, P. Blier, RSM, SG), Janssen Ortho (KK MK, P. Blier, PC, RSM), Labopharm (P. Blier), Litebook Company (RWL), Lundbeck (KK, KR, MK, P. Bleau, P. Blier, PC, RWL, RSM, SG), Lundbeck Institute (RWL), Organon (MK, RSM), Merck (P. Blier, RSM), Mochida (RWL), Otsuka (KK), Pfizer (P. Bleau, P. Blier, PC, RWL, RSM, SG), Pierre Fabre (P. Blier), Purdue (IS), Servier (P. Blier, RWL, SG), Shire (MK, RSM, IS), Solvay (MK), St. Jude's Medical (RWL), Sunovion (KK, P. Blier), Takeda (P. Blier, RWL), UBC Institute of Mental Health/Coast Capital Savings (RWL), Valeant (P. Blier, IS), Wyeth (P. Bleau, MK, SG)

Consultation fees: AstraZeneca (MK), BMS (MK), Boehringer Ingelheim (MK), Clinique et Développement In Virtuo Inc. (SB), Eli Lilly Canada (MK, SG),
GlaxoSmithKline (MK, SG), Janssen Ortho (MK), Lundbeck (MK, PR, SG), Organon (MK), Pfizer (SG), Servier (SG), Shire (MK), Solvay (MK), Wyeth (MK, SG)

Educational support: Astra Zeneca (RSM), BMS (RSM), CME Outfitters (RSM), Eli Lilly Canada (RSM, IS), France Foundation (RSM), ISCME (RSM), Merck (RSM), Optum Health (RSM), Pfizer (RSM), Physicians' Postgraduate Press (RSM), Shire (IS)

Research grants/clinical trial funding: AstraZeneca (KK, MK, P. Bleau, RSM RWL), Biovail (RWL), BMS (KK, P. Bleau, RWL), Canadian Foundation for Innovation (MK), Canadian Institutes of Health Research (CIHR) (MK, RWL, SG), Canadian Network for Mood and Anxiety Treatments (CANMAT) (RWL), Canadian Psychiatric Association (CPA) Foundation (MK, RWL), Centre for Addiction and Mental Health Foundation (MK), CR Younger Foundation (SG), Eli Lilly Canada (MK, P. Bleau, PR, RSM, RWL), Genuine Health (MK), GlaxoSmithKline (MK), Janssen Ortho (MK, RSM), Litebook Company (RWL), Lundbeck (KK, MK, P. Bleau, RSM, RWL), Lundbeck Institute (RWL), Mochida (RWL), National Alliance for Research on Schizophrenia and Depression (NARSAD) (RSM), National Institutes of Mental Health (NIMH) (RSM), Ontario Ministry of Health (SG), Ontario Mental Health Foundation (SG), Organon (MK), Pfizer (P. Bleau, RSM, RWL), Roche (PR), Servier (RWL), Sick Kids Foundation (MK), Solvay (MK), St. Jude's Medical (RWL), Shire (MK, RSM), Stanley Medical Research Institute (RSM), Takeda (RWL), UBC Institute of Mental Health/Coast Capital Savings (RWL), Wyeth (MK, P. Bleau) Unrestricted grants: Astra Zeneca Canada (MK), Eli Lilly Canada (MK), Janssen Inc. (MK), Lundbeck Canada (MK), Pfizer (MK), Purdue Pharma (MK), Servier Canada (MK), Shire Canada (MK), Valeant Canada (MK)

Reimbursements, fees, funding, or salary: In the past five years, MVA received reimbursements, fees, funding, or salary from: Astra Zeneca, Biovail, Canadian Foundation for Innovation (CFI), Cephalon, Eli Lilly, Forest Laboratories, GlaxoSmithKline, Hamilton Academic Health Sciences Organization (HAHSO) Innovation Grant (AFP Innovation Grant), Janssen Ortho, Labo Pharm, Lundbeck, National Institutes of Health (NIH), Novartis, Pfizer Inc., Servier, Shire, Sunovion, Valeant, Wyeth-Ayerst

Stock/share ownership: Clinique et Développement In Virtuo Inc. (SB)

\section{Authors' contributions}

We thank all co-authors for their considerable expertise in generating these guidelines. Authors who were members of the executive committee (MK, PB, $\mathrm{PB}, \mathrm{PC}, \mathrm{KK}, \mathrm{MVA}$ ) took part in teleconferences and a meeting in December 2012 to reach consensus on the strength of evidence and treatment recommendations. Draft guidelines were then developed by the core committee and revised by all co-authors. The entire content was subsequently circulated to all members of the Canadian Anxiety Guidelines Initiative Group for additional comments and approval during 2013. GA, LKK, RWL, RSM, and IS provided additional reviews of the comorbidity section. The final manuscript was then circulated to external reviewers (MP, DS, LDM) and revisions were made based on input from the core committee.

\section{Acknowledgements}

The consensus group would like to thank Astra Zeneca Canada, Eli-Lilly Canada, Janssen Inc., Lundbeck Canada, Pfizer Canada, Purdue Canada, Servier Canada Inc., Shire Canada, and Valeant Canada for their generous support of the guideline process with unrestricted educational grants. Funds were used for editorial assistance and meeting logistics; none of the members received payment for participating in the guideline development process. The consensus group would also like to thank Pauline Lavigne and Steven Portelance who provided medical writing services on their behalf.

\section{Declarations}

The development and publication of these guidelines was supported by unrestricted educational grants provided by Astra Zeneca Canada, Eli-Lilly Canada, Janssen Inc., Lundbeck Canada, Pfizer Canada, Purdue Canada, Servier Canada Inc., Shire Canada, and Valeant Canada. None of the members received payment for participating in the development of these guidelines. This article has been published as part of BMC Psychiatry Volume 14 Supplement 1, 2014: Canadian Anxiety Disorders Guidelines Initiative: Clinical practice guidelines for the management of anxiety, posttraumatic stress and obsessive-compulsive disorders. The full contents of the supplement are available online at http://www.biomedcentral.com/bmcpsychiatry/ supplements/14/S1. 


\section{Authors' details}

'Department of Psychiatry, University of Toronto, Toronto, ON, M5S 1A1, Canada. ${ }^{2}$ Department of Psychiatry, McGill University, Montreal, QC, H3A 1A1, Canada. ${ }^{3}$ Department of Psychiatry and Cellular/Molecular Medicines, University of Ottawa, Ottawa, ON, K1Z 7K4, Canada. ${ }^{4}$ Department of Psychiatry, University of Alberta, Edmonton, AB, T6G 2R7, Canada. ${ }^{5}$ Department of Psychiatry, University of British Columbia, Vancouver, BC, V6T 2A1, Canada. ${ }^{6}$ Department of Psychiatry and Behavioural Neuroscience, McMaster University, Hamilton, ON, L8N 3K7, Canada.

Published: 2 July 2014

\section{References}

1. Kessler RC, Angermeyer M, Anthony JC, R DG, Demyttenaere K, Gasquet I, G DG, Gluzman S, Gureje O, Haro JM, et al: Lifetime prevalence and ageof-onset distributions of mental disorders in the World Health Organization's World Mental Health Survey Initiative. World Psychiatry 2007, 6:168-176.

2. Kessler RC, Berglund P, Demler O, Jin R, Merikangas KR, Walters EE: Lifetime prevalence and age-of-onset distributions of DSM-IV disorders in the National Comorbidity Survey Replication. Arch Gen Psychiatry 2005, 62:593-602

3. Kessler RC, Chiu WT, Demler O, Merikangas KR, Walters EE: Prevalence, severity, and comorbidity of 12-month DSM-IV disorders in the National Comorbidity Survey Replication. Arch Gen Psychiatry 2005, 62:617-627.

4. Somers JM, Goldner EM, Waraich P, Hsu L: Prevalence and incidence studies of anxiety disorders: a systematic review of the literature. Can J Psychiatry 2006, 51:100-113.

5. Martin-Merino E, Ruigomez A, Wallander MA, Johansson S, GarciaRodriguez LA: Prevalence, incidence, morbidity and treatment patterns in a cohort of patients diagnosed with anxiety in UK primary care. Fam Pract 2010, 27:9-16.

6. Vermani M, Marcus M, Katzman MA: Rates of detection of mood and anxiety disorders in primary care: a descriptive, cross-sectional study. Prim Care Companion CNS Disord 2011, 13, doi 10.4088/PCC.4010m01013.

7. Kroenke K, Spitzer RL, Williams JB, Monahan PO, Lowe B: Anxiety disorders in primary care: prevalence, impairment, comorbidity, and detection. Ann Intern Med 2007, 146:317-325.

8. Weisberg RB, Dyck I, Culpepper L, Keller MB: Psychiatric treatment in primary care patients with anxiety disorders: a comparison of care received from primary care providers and psychiatrists. Am J Psychiatry 2007, 164:276-282.

9. McLean CP, Asnaani A, Litz BT, Hofmann SG: Gender differences in anxiety disorders: prevalence, course of illness, comorbidity and burden of illness. J Psychiatr Res 2011, 45:1027-1035.

10. Stein M, Fuetsch M, Muller N, Hofler M, Lieb R, Wittchen H: Social anxiety disorder and the risk of depression: a prospective community study of adolescents and young adults. Arch Gen Psychiatry 2001, 58:251-256.

11. Pine DS, Cohen P, Gurley D, Brook J, Ma Y: The risk for early-adulthood anxiety and depressive disorders in adolescents with anxiety and depressive disorders. Arch Gen Psychiatry 1998, 55:56-64.

12. Wittchen HU, Kessler RC, Pfister H, Lieb M: Why do people with anxiety disorders become depressed? A prospective-longitudinal community study. Acta Psychiatr Scand Suppl 2000, 14-23.

13. Senaratne R, Van Ameringen M, Mancini C, Patterson B: The burden of anxiety disorders on the family. J Nerv Ment Dis 2010, 198:876-880.

14. Erickson SR, Guthrie S, Vanetten-Lee M, Himle J, Hoffman J, Santos SF, Janeck AS, Zivin K, Abelson JL: Severity of anxiety and work-related outcomes of patients with anxiety disorders. Depress Anxiety 2009, 26:1165-1171

15. Sherbourne CD, Sullivan G, Craske MG, Roy-Byrne P, Golinelli D, Rose RD, Chavira DA, Bystritsky A, Stein MB: Functioning and disability levels in primary care out-patients with one or more anxiety disorders. Psychol Med 2010, 40:2059-2068.

16. Comer JS, Blanco C, Hasin DS, Liu SM, Grant BF, Turner JB, Olfson M: Health-related quality of life across the anxiety disorders: results from the national epidemiologic survey on alcohol and related conditions (NESARC). J Clin Psychiatry 2011, 72:43-50.

17. Barrera TL, Norton PJ: Quality of life impairment in generalized anxiety disorder, social phobia, and panic disorder. J Anxiety Disord 2009, 23:1086-1090
18. Wittchen HU: Generalized anxiety disorder: prevalence, burden, and cost to society. Depress Anxiety 2002, 16:162-171.

19. Waghorn G, Chant D, White P, Whiteford H: Disability, employment and work performance among people with ICD-10 anxiety disorders. Aust $N$ Z J Psychiatry 2005, 39:55-66.

20. Nepon J, Belik SL, Bolton J, Sareen J: The relationship between anxiety disorders and suicide attempts: findings from the National Epidemiologic Survey on Alcohol and Related Conditions. Depress Anxiety 2010, 27:791-798.

21. Bolton JM, Cox BJ, Afifi TO, Enns MW, Bienvenu OJ, Sareen J: Anxiety disorders and risk for suicide attempts: findings from the Baltimore Epidemiologic Catchment area follow-up study. Depress Anxiety 2008, 25:477-481.

22. Sareen J, Cox BJ, Afifi TO, de Graaf R, Asmundson GJ, ten Have M, Stein MB: Anxiety disorders and risk for suicidal ideation and suicide attempts: a population-based longitudinal study of adults. Arch Gen Psychiatry 2005, 62:1249-1257.

23. Cougle JR, Keough ME, Riccardi CJ, Sachs-Ericsson N: Anxiety disorders and suicidality in the National Comorbidity Survey-Replication. J Psychiatr Res 2009, 43:825-829.

24. Pfeiffer PN, Ganoczy D, Ilgen M, Zivin K, Valenstein M: Comorbid anxiety as a suicide risk factor among depressed veterans. Depress Anxiety 2009, 26:752-757

25. Schaffer A, Levitt A, Bagby R, Kennedy S, Levitan R, Joffe R: Suicidal ideation in major depression: sex differences and impact of comorbid anxiety. Can J Psychiatry 2000, 45:822-826.

26. American Psychiatric Association: Diagnostic and Statistical Manual of Mental Disorders (DSM-5). Washington, DC: American Psychiatric Association; Fifth 2013.

27. Van Ameringen M, Pollack M: Generalized Anxiety Disorder (Oxford Psychiatry Library). New York, NY: Oxford University Press; 2010.

28. Connor K, Kobak K, Churchill L, Katzelnick D, Davidson J: Mini-SPIN: A brief screening assessment for generalized social anxiety disorder. Depress Anxiety 2001, 14:137-140.

29. Mancini C, Van Ameringen M, Pipe B, Oakman J: Development and validation of self-report psychiatric screening tool: MACSCREEN [poster]. Anxiety Disorders Association of America 23rd Annual Conference; March 27-30; Toronto, Canada 2003.

30. Van Ameringen M, Mancini C, Simpson W, Patterson B: Potential use of Internet-based screening for anxiety disorders: a pilot study. Depress Anxiety 2010, 27:1006-1010.

31. Ballenger J, Davidson J, Lecrubier Y, Nutt D, Borkovec T, Rickels K, Stein D, Wittchen $\mathrm{H}$ : Consensus statement on generalized anxiety disorder from the International Consensus Group on Depression and Anxiety. J Clin Psychiatry 2001, 62(Suppl 11):53-58.

32. Swinson R, Antony M, Bleau P, Chokka P, Craven M, Fallu A, Kjernisted K, Lanius R, Manassis K, McIntosh D, et al: Clinical practice guidelines. Management of anxiety disorders. Can J Psychiatry 2006, 51:95-91S

33. Milne BJ, Caspi A, Harrington $H$, Poulton R, Rutter M, Moffitt TE: Predictive value of family history on severity of illness: the case for depression, anxiety, alcohol dependence, and drug dependence. Arch Gen Psychiatry 2009, 66:738-747.

34. Batelaan NM, Smit F, de Graaf R, van Balkom AJ, Vollebergh WA, Beekman AT: Identifying target groups for the prevention of anxiety disorders in the general population. Acta Psychiatr Scand 2010, 122:56-65.

35. Karsten J, Hartman CA, Smit JH, Zitman FG, Beekman AT, Cuijpers P, van der Does AJ, Ormel J, Nolen WA, Penninx BW: Psychiatric history and subthreshold symptoms as predictors of the occurrence of depressive or anxiety disorder within 2 years. Br J Psychiatry 2011, 198:206-212.

36. McLaughlin KA, Hatzenbuehler ML: Stressful life events, anxiety sensitivity, and internalizing symptoms in adolescents. J Abnorm Psychol 2009, 118:659-669.

37. Chu DA, Williams LM, Harris AW, Bryant RA, Gatt JM: Early life trauma predicts self-reported levels of depressive and anxiety symptoms in nonclinical community adults: Relative contributions of early life stressor types and adult trauma exposure. J Psychiatr Res 2013, 47:23-32.

38. Flensborg-Madsen T, Tolstrup J, Sorensen HJ, Mortensen EL: Social and psychological predictors of onset of anxiety disorders: results from a large prospective cohort study. Soc Psychiatry Psychiatr Epidemiol 2012, 47:711-721. 
39. Heider D, Matschinger $H$, Bernert $S$, Alonso J, Brugha TS, Bruffaerts $R$, de Girolamo G, Dietrich S, Angermeyer MC: Adverse parenting as a risk factor in the occurrence of anxiety disorders : a study in six European countries. Soc Psychiatry Psychiatr Epidemiol 2008, 43:266-272.

40. Strine TW, Mokdad AH, Balluz LS, Gonzalez O, Crider R, Berry JT, Kroenke K Depression and anxiety in the United States: findings from the 2006 Behavioral Risk Factor Surveillance System. Psychiatr Serv 2008, 59:1383-1390.

41. Kagan J, Snidman N: Early childhood predictors of adult anxiety disorders. Biol Psychiatry 1999, 46:1536-1541.

42. Muris $P$, van Brakel AM, Arntz A, Schouten E: Behavioral inhibition as a risk factor for the development of childhood anxiety disorders: A longitudinal study. J Child Fam Stud 2011, 20:157-170.

43. Schaffer A, Cairney J, Cheung A, Veldhuizen S, Levitt A: Community survey of bipolar disorder in Canada: lifetime prevalence and illness characteristics. Can J Psychiatry 2006, 51:9-16.

44. Kessler RC, Berglund P, Demler O, Jin R, Koretz D, Merikangas KR, Rush AJ, Walters EE, Wang PS: The epidemiology of major depressive disorder: results from the National Comorbidity Survey Replication (NCS-R). JAMA 2003, 289:3095-3105.

45. Kessler RC, Adler L, Barkley R, Biederman J, Conners CK, Demler O, Faraone SV, Greenhill LL, Howes MJ, Secnik K, et al: The prevalence and correlates of adult ADHD in the United States: results from the National Comorbidity Survey Replication. Am J Psychiatry 2006, 163:716-723.

46. Klein Hofmeijer-Sevink M, Batelaan NM, van Megen HJ, Penninx BW, Cath DC, van den Hout MA, van Balkom AJ: Clinical relevance of comorbidity in anxiety disorders: a report from the Netherlands Study of Depression and Anxiety (NESDA). J Affect Disord 2012, 137:106-112.

47. Bruce S, Machan J, Dyck I, Keller M: Infrequency of "pure" GAD: impact of psychiatric comorbidity on clinical course. Depress Anxiety 2001, 14:219-225.

48. Shankman S, Klein D: The impact of comorbid anxiety disorders on the course of dysthymic disorder: a 5-year prospective longitudinal study. J Affect Disord 2002, 70:211-217.

49. Boylan K, Bieling P, Marriott M, Begin H, Young L, MacQueen G: Impact of comorbid anxiety disorders on outcome in a cohort of patients with bipolar disorder. J Clin Psychiatry 2004, 65:1106-1113.

50. Mackenzie CS, Reynolds K, Cairney J, Streiner DL, Sareen J: Disorder-specific mental health service use for mood and anxiety disorders: associations with age, sex, and psychiatric comorbidity. Depress Anxiety 2012, 29:234-242

51. McLaughlin T, Geissler E, Wan G: Comorbidities and associated treatment charges in patients with anxiety disorders. Pharmacotherapy 2003, 23:1251-1256

52. Sareen J, Jacobi F, Cox BJ, Belik SL, Clara I, Stein MB: Disability and poor quality of life associated with comorbid anxiety disorders and physical conditions. Arch Intern Med 2006, 166:2109-2116.

53. Yatham LN, Kennedy SH, Schaffer A, Parikh SV, Beaulieu S, O'Donovan C, MacQueen G, Mclntyre RS, Sharma V, Ravindran A, et al: Canadian Network for Mood and Anxiety Treatments (CANMAT) and International Society for Bipolar Disorders (ISBD) collaborative update of CANMAT guidelines for the management of patients with bipolar disorder: update 2009. Bipolar Disord 2009, 11:225-255.

54. van Balkom AJ, Bakker A, Spinhoven P, Blaauw BM, Smeenk S, Ruesink B: A meta-analysis of the treatment of panic disorder with or without agoraphobia: a comparison of psychopharmacological, cognitivebehavioral, and combination treatments. J Nerv Ment Dis 1997, 185:510-516.

55. Siev J, Chambless DL: Specificity of treatment effects: cognitive therapy and relaxation for generalized anxiety and panic disorders. J Consult Clin Psychol 2007, 75:513-522.

56. Sanchez-Meca J, Rosa-Alcazar Al, Marin-Martinez F, Gomez-Conesa A: Psychological treatment of panic disorder with or without agoraphobia: a meta-analysis. Clin Psychol Rev 2010, 30:37-50.

57. Wolitzky-Taylor KB, Horowitz JD, Powers MB, Telch MJ: Psychological approaches in the treatment of specific phobias: a meta-analysis. Clin Psychol Rev 2008, 28:1021-1037.

58. Fedoroff I, Taylor S: Psychological and pharmacological treatments of social phobia: a meta-analysis. J Clin Psychopharmacol 2001, 21:311-324

59. Acarturk C, Cuijpers P, van Straten A, de Graaf R: Psychological treatment of social anxiety disorder: a meta-analysis. Psychol Med 2009, 39:241-254.
60. Rosa-Alcazar Al, Sanchez-Meca J, Gomez-Conesa A, Marin-Martinez F: Psychological treatment of obsessive-compulsive disorder: a metaanalysis. Clin Psychol Rev 2008, 28:1310-1325.

61. Jonsson $\mathrm{H}$, Hougaard E: Group cognitive behavioural therapy for obsessive-compulsive disorder: a systematic review and meta-analysis. Acta Psychiatr Scand 2009, 119:98-106.

62. Gava I, Barbui C, Aguglia E, Carlino D, Churchill R, De Vanna M, McGuire HF: Psychological treatments versus treatment as usual for obsessive compulsive disorder (OCD). Cochrane Database Syst Rev 2007, CD005333

63. Ougrin D: Efficacy of exposure versus cognitive therapy in anxiety disorders: systematic review and meta-analysis. BMC Psychiatry 2011, 11:200.

64. Borkovec T, Ruscio A: Psychotherapy for generalized anxiety disorder. J Clin Psychiatry 2001, 62(Suppl 11):37-42, discussion 43-35.

65. Hunot V, Churchill R, Silva de Lima M, Teixeira V: Psychological therapies for generalised anxiety disorder. Cochrane Database Syst Rev 2007, CD001848.

66. Bisson J, Andrew M: Psychological treatment of post-traumatic stress disorder (PTSD). Cochrane Database Syst Rev 2007, CD003388.

67. Bisson J, Ehlers A, Matthews R, Pilling S, Richards D, Turner S: Psychological treatments for chronic post-traumatic stress disorder. Systematic review and meta-analysis. Br J Psychiatry 2007, 190:97-104.

68. Seidler GH, Wagner FE: Comparing the efficacy of EMDR and traumafocused cognitive-behavioral therapy in the treatment of PTSD: a metaanalytic study. Psychol Med 2006, 36:1515-1522.

69. Powers MB, Halpern JM, Ferenschak MP, Gillihan SJ, Foa EB: A metaanalytic review of prolonged exposure for posttraumatic stress disorder. Clin Psychol Rev 2010, 30:635-641.

70. Hofmann SG, Smits JA: Cognitive-behavioral therapy for adult anxiety disorders: a meta-analysis of randomized placebo-controlled trials. J Clin Psychiatry 2008, 69:621-632.

71. Roshanaei-Moghaddam B, Pauly MC, Atkins DC, Baldwin SA, Stein MB, RoyByrne P: Relative effects of CBT and pharmacotherapy in depression versus anxiety: is medication somewhat better for depression, and CBT somewhat better for anxiety? Depress Anxiety 2011, 28:560-567.

72. Hofmann SG, Sawyer AT, Witt AA, Oh D: The effect of mindfulness-based therapy on anxiety and depression: A meta-analytic review. J Consult Clin Psychol 2010, 78:169-183.

73. Boschen MJ, Oei TP: A cognitive behavioral case formulation framework for treatment planning in anxiety disorders. Depress Anxiety 2008, 25:811-823.

74. Coull G, Morris PG: The clinical effectiveness of CBT-based guided selfhelp interventions for anxiety and depressive disorders: a systematic review. Psychol Med 2011, 41:2239-2252.

75. Lewis C, Pearce J, Bisson J: Efficacy, cost-effectiveness and acceptability of self-help interventions for anxiety disorders: systematic review. $\mathrm{Br} J$ Psychiatry 2012, 200:15-21.

76. Lucock M, Padgett K, Noble R, Westley A, Atha C, Horsefield C, Leach C: Controlled clinical trial of a self-help for anxiety intervention for patients waiting for psychological therapy. Behav Cog Psychother 2008, 36:541-551.

77. Craske MG, Rose RD, Lang A, Welch SS, Campbell-Sills L, Sullivan G, Sherbourne C, Bystritsky A, Stein MB, Roy-Byrne PP: Computer-assisted delivery of cognitive behavioral therapy for anxiety disorders in primary-care settings. Depress Anxiety 2009, 26:235-242.

78. Cavanagh K, Seccombe N, Lidbetter N: The implementation of computerized cognitive behavioural therapies in a service user-led, third sector self help clinic. Behav Cogn Psychother 2011, 39:427-442.

79. Titov N, Andrews G, Johnston L, Robinson E, Spence J: Transdiagnostic Internet treatment for anxiety disorders: a randomized controlled trial. Behav Res Ther 2010, 48:890-899.

80. Opris D, Pintea S, Garcia-Palacios A, Botella C, Szamoskozi S, David D: Virtual reality exposure therapy in anxiety disorders: a quantitative meta-analysis. Depress Anxiety 2012, 29:85-93.

81. Powers MB, Emmelkamp PM: Virtual reality exposure therapy for anxiety disorders: a meta-analysis. J Anxiety Disord 2008, 22:561-569.

82. Bandelow B, Seidler-Brandler U, Becker A, Wedekind D, Ruther E: Metaanalysis of randomized controlled comparisons of psychopharmacological and psychological treatments for anxiety disorders. World J Biol Psychiatry 2007, 8:175-187. 
83. Hofmann SG, Sawyer AT, Korte KJ, Smits JA: Is it beneficial to add pharmacotherapy to cognitive-behavioral therapy when treating anxiety disorders? a meta-analytic review. Int J Cogn Ther 2009, 2:160-175.

84. Drug product database. [http://webprod5.hc-sc.gc.ca/dpd-bdpp/indexeng.jsp].

85. Practice guideline for the treatment of patients with panic disorder. [http://psychiatryonline.org/content.aspx?bookid=28\&sectionid=1680635].

86. Brambilla P, Cipriani A, Hotopf M, Barbui C: Side-effect profile of fluoxetine in comparison with other SSRIs, tricyclic and newer antidepressants: a meta-analysis of clinical trial data. Pharmacopsychiatry 2005, 38:69-77

87. Hu X, Bull S, Hunkeler E, Ming E, Lee J, Fireman B, Markson L: Incidence and duration of side effects and those rated as bothersome with selective serotonin reuptake inhibitor treatment for depression: patient report versus physician estimate. J Clin Psychiatry 2004, 65:959-965.

88. Cascade E, Kalali AH, Kennedy SH: Real-world data on SSRI antidepressant side effects. Psychiatry (Edgmont) 2009, 6:16-18.

89. Hirschfeld R: Long-term side effects of SSRIs: sexual dysfunction and weight gain. J Clin Psychiatry 2003, 64(Suppl 18):20-24.

90. Dall M, Schaffalitzky de Muckadell OB, Lassen AT, Hansen JM, Hallas J: An association between selective serotonin reuptake inhibitor use and serious upper gastrointestinal bleeding. Clin Gastroenterol Hepatol 2009, 7:1314-1321.

91. de Abajo FJ, Garcia-Rodriguez LA: Risk of upper gastrointestinal tract bleeding associated with selective serotonin reuptake inhibitors and venlafaxine therapy: interaction with nonsteroidal anti-inflammatory drugs and effect of acid-suppressing agents. Arch Gen Psychiatry 2008, 65:795-803.

92. Haney EM, Chan BK, Diem SJ, Ensrud KE, Cauley JA, Barrett-Connor E, Orwoll E, Bliziotes MM: Association of low bone mineral density with selective serotonin reuptake inhibitor use by older men. Arch Intern Med 2007, 167:1246-1251.

93. Williams LJ, Henry MJ, Berk M, Dodd S, Jacka FN, Kotowicz MA Nicholson GC, Pasco JA: Selective serotonin reuptake inhibitor use and bone mineral density in women with a history of depression. Int Clin Psychopharmacol 2008, 23:84-87.

94. Eom CS, Lee HK, Ye S, Park SM, Cho KH: Use of selective serotonin reuptake inhibitors and risk of fracture: a systematic review and metaanalysis. J Bone Miner Res 2012, 27:1186-1195.

95. Smith JM: Clinical implications of treating depressed older adults with SSRIs: possible risk of hyponatremia. J Gerontol Nurs 2010, 36:22-27, quiz 28-29.

96. Shelton RC: The nature of the discontinuation syndrome associated with antidepressant drugs. J Clin Psychiatry 2006, 67(Suppl 4):3-7.

97. Kondro W: UK bans, Health Canada warns about antidepressants. CMAJ 2004, 171:23.

98. Labeling change request letter for antidepressant medications. [http:// www.fda.gov/Drugs/DrugSafety/InformationbyDrugClass/ucm096352.htm]

99. Barbui C, Esposito E, Cipriani A: Selective serotonin reuptake inhibitors and risk of suicide: a systematic review of observational studies. CMAJ 2009, 180:291-297.

100. Olmer A, lancu I, Strous RD: Exposure to antidepressant medications and suicide attempts in adult depressed inpatients. J Nerv Ment Dis 2012 200:531-534.

101. Degner D, Grohmann R, Bleich S, Ruther E: New antidepressant drugs. What side effects and interactions are to be expected? MMW Fortschr Med 2000, 142:35-38, 40.

102. Chouinard G: Issues in the clinical use of benzodiazepines: potency, withdrawal, and rebound. J Clin Psychiatry 2004, 65(Suppl 5):7-12.

103. Ciraulo DA, Nace EP: Benzodiazepine treatment of anxiety or insomnia in substance abuse patients. Am J Addict 2000, 9:276-279, discussion 280274.

104. Petrovic M, Mariman A, Warie $H$, Afschrift M, Pevernagie D: Is there a rationale for prescription of benzodiazepines in the elderly? Review of the literature. Acta Clin Belg 2003, 58:27-36

105. Allain H, Bentue-Ferrer D, Polard E, Akwa Y, Patat A: Postural instability and consequent falls and hip fractures associated with use of hypnotics in the elderly: a comparative review. Drugs Aging 2005, 22:749-765.
106. Barker MJ, Greenwood KM, Jackson M, Crowe SF: Cognitive effects of long-term benzodiazepine use: a meta-analysis. CNS Drugs 2004, 18:37-48.

107. Barker MJ, Greenwood KM, Jackson M, Crowe SF: An evaluation of persisting cognitive effects after withdrawal from long-term benzodiazepine use. J Int Neuropsychol Soc 2005, 11:281-289.

108. Chessick CA, Allen MH, Thase M, Batista Miralha da Cunha AB, Kapczinski FF, de Lima MS, dos Santos Souza JJ: Azapirones for generalized anxiety disorder. Cochrane Database Syst Rev 2006, 3 CD006115

109. Baker RA, Pikalov A, Tran QV, Kremenets T, Arani RB, Doraiswamy PM: Atypical antipsychotic drugs and diabetes mellitus in the US Food and Drug Administration Adverse Event database: a systematic Bayesian signal detection analysis. Psychopharmacol Bull 2009, 42:11-31.

110. Yatham LN, Kennedy SH, O'Donovan C, Parikh SV, MacQueen G, McIntyre RS, Sharma V, Beaulieu S: Canadian Network for Mood and Anxiety Treatments (CANMAT) guidelines for the management of patients with bipolar disorder: update 2007. Bipolar Disord 2006, 8:721-739.

111. Yatham LN, Kennedy SH, O'Donovan C, Parikh S, MacQueen G, Mclntyre R, Sharma V, Silverstone P, Alda M, Baruch P, et al: Canadian Network for Mood and Anxiety Treatments (CANMAT) guidelines for the management of patients with bipolar disorder: consensus and controversies. Bipolar Disord 2005, 7(Suppl 3):5-69.

112. Sumiyoshi T, Roy A, Anil AE, Jayathilake K, Ertugrul A, Meltzer HY: A comparison of incidence of diabetes mellitus between atypical antipsychotic drugs: a survey for clozapine, risperidone, olanzapine, and quetiapine. J Clin Psychopharmacol 2004, 24:345-348.

113. Citrome LL, Holt RI, Zachry WM, Clewell JD, Orth PA, Karagianis JL, Hoffmann VP: Risk of treatment-emergent diabetes mellitus in patients receiving antipsychotics. Ann Pharmacother 2007, 41:1593-1603.

114. Olfson M, Marcus SC, Corey-Lisle P, Tuomari AV, Hines P, L'Italien GJ: Hyperlipidemia following treatment with antipsychotic medications. Am J Psychiatry 2006, 163:1821-1825.

115. Depping AM, Komossa K, Kissling W, Leucht S: Second-generation antipsychotics for anxiety disorders. Cochrane Database Syst Rev 2010, CD008120

116. LaLonde CD, Van Lieshout RJ: Treating generalized anxiety disorder with second generation antipsychotics: a systematic review and metaanalysis. J Clin Psychopharmacol 2011, 31:326-333.

117. Torrent C, Martinez-Aran A, Daban C, Amann B, Balanza-Martinez V, Del Mar Bonnin C, Cruz N, Franco C, Tabares-Seisdedos R, Vieta E: Effects of atypical antipsychotics on neurocognition in euthymic bipolar patients. Compr Psychiatry 2011, 52:613-622.

118. Correll CU, Sheridan EM, DelBello MP: Antipsychotic and mood stabilizer efficacy and tolerability in pediatric and adult patients with bipolar I mania: a comparative analysis of acute, randomized, placebo-controlled trials. Bipolar Disord 2010, 12:116-141.

119. Doyle A, Pollack M: Establishment of remission criteria for anxiety disorders. J Clin Psychiatry 2003, 64(Suppl 15):40-45.

120. Kjernisted KD, Bleau P: Long-term goals in the management of acute and chronic anxiety disorders. Can J Psychiatry 2004, 49:51S-63S.

121. Kessler RC, Chiu WT, Jin R, Ruscio AM, Shear K, Walters EE: The epidemiology of panic attacks, panic disorder, and agoraphobia in the National Comorbidity Survey Replication. Arch Gen Psychiatry 2006, 63:415-424.

122. Grant BF, Hasin DS, Stinson FS, Dawson DA, Goldstein RB, Smith S, Huang B, Saha TD: The epidemiology of DSM-IV panic disorder and agoraphobia in the United States: results from the National Epidemiologic Survey on Alcohol and Related Conditions. J Clin Psychiatry 2006, 67:363-374.

123. Kinley DJ, Cox BJ, Clara I, Goodwin RD, Sareen J: Panic attacks and their relation to psychological and physical functioning in Canadians: results from a nationally representative sample. Can J Psychiatry 2009, 54:113-122.

124. Goodwin RD, Lieb R, Hoefler M, Pfister H, Bittner A, Beesdo K, Wittchen $\mathrm{HU}$ : Panic attack as a risk factor for severe psychopathology. Am J Psychiatry 2004, 161:2207-2214.

125. Kjernisted K, Mclntosh D: Venlafaxine extended release (XR) in the treatment of panic disorder. Ther Clin Risk Manag 2007, 3:59-69. 
126. Culpepper L: Identifying and treating panic disorder in primary care. J Clin Psychiatry 2004, 65(Suppl 5):19-23.

127. Craske MG, Tsao JC: Assessment and treatment of nocturnal panic attacks. Sleep Med Rev 2005, 9:173-184.

128. Romans S, Cohen M, Forte T: Rates of depression and anxiety in urban and rural Canada. Soc Psychiatry Psychiatr Epidemiol 2011, 46:567-575.

129. Galderisi S, Mancuso F, Mucci A, Garramone S, Zamboli R, Maj M: Alexithymia and cognitive dysfunctions in patients with panic disorder. Psychother Psychosom 2008, 77:182-188.

130. Deckersbach T, Moshier SJ, Tuschen-Caffier B, Otto MW: Memory dysfunction in panic disorder: an investigation of the role of chronic benzodiazepine use. Depress Anxiety 2011, 28:999-1007.

131. Pastucha P, Prasko J, Grambal A, Latalova K, Sigmundova Z, Sykorova T, Tichackova A: Panic disorder and dissociation - comparison with healthy controls. Neuro Endocrinol Lett 2009, 30:774-778.

132. Kessler H, Roth J, von Wietersheim J, Deighton RM, Traue HC: Emotion recognition patterns in patients with panic disorder. Depress Anxiety 2007, 24:223-226.

133. Tull MT, Roemer L: Emotion regulation difficulties associated with the experience of uncued panic attacks: evidence of experiential avoidance, emotional nonacceptance, and decreased emotional clarity. Behav Ther 2007, 38:378-391.

134. Batelaan N, Smit F, de Graaf R, van Balkom A, Vollebergh W, Beekman A: Economic costs of full-blown and subthreshold panic disorder. $J$ Affect Disord 2007, 104:127-136.

135. Gros DF, Frueh BC, Magruder KM: Prevalence and features of panic disorder and comparison to posttraumatic stress disorder in VA primary care. Gen Hosp Psychiatry 2011, 33:482-488.

136. de Graaf R, Tuithof M, van Dorsselaer S, Ten Have M: Comparing the effects on work performance of mental and physical disorders. Soc Psychiatry Psychiatr Epidemiol 2012, 47:1873-1883.

137. Mathew AR, Norton PJ, Zvolensky MJ, Buckner JD, Smits JA: Smoking behavior and alcohol consumption in individuals with panic attacks. J Cogn Psychother 2011, 25:61-70.

138. Bienvenu OJ, Onyike CU, Stein MB, Chen LS, Samuels J, Nestadt G, Eaton WW: Agoraphobia in adults: incidence and longitudinal relationship with panic. Br J Psychiatry 2006, 188:432-438.

139. Korczak DJ, Goldstein BI, Levitt AJ: Panic disorder, cardiac diagnosis and emergency department utilization in an epidemiologic community sample. Gen Hosp Psychiatry 2007, 29:335-339.

140. Senaratne R, Van Ameringen M, Mancini C, Patterson B, Bennett M: The prevalence of migraine headaches in an anxiety disorders clinic sample. CNS Neurosci Ther 2010, 16:76-82.

141. Yamada K, Moriwaki K, Oiso H, Ishigooka J: High prevalence of comorbidity of migraine in outpatients with panic disorder and effectiveness of psychopharmacotherapy for both disorders: a retrospective open label study. Psychiatry Res 2011, 185:145-148.

142. Marshall EC, Zvolensky MJ, Sachs-Ericsson N, Schmidt NB, Bernstein A: Panic attacks and physical health problems in a representative sample: singular and interactive associations with psychological problems, and interpersonal and physical disability. J Anxiety Disord 2008, 22:78-87.

143. Craske MG, Kircanski K, Epstein A, Wittchen HU, Pine DS, LewisFernandez R, Hinton D: Panic disorder: a review of DSM-IV panic disorder and proposals for DSM-V. Depress Anxiety 2010, 27:93-112.

144. American Psychiatric Association: Diagnostic and Statistical Manual of Mental Disorders, text revision (DSM-IV-TR). Washington, DC: American Psychiatric Association; Fourth 2000

145. Wittchen HU, Gloster AT, Beesdo-Baum K, Fava GA, Craske MG: Agoraphobia: a review of the diagnostic classificatory position and criteria. Depress Anxiety 2010, 27:113-133.

146. Clum GA, Surls R: A meta-analysis of treatments for panic disorder. J Consult Clin Psychol 1993, 61:317-326.

147. Gould R, Otto M, Pollack M: A meta-analysis of treatment outcome for panic disorder. Clin Psychol Psychother 1995, 15:819-844.

148. Marchand A, Roberge P, Primiano S, Germain V: A randomized, controlled clinical trial of standard, group and brief cognitive-behavioral therapy for panic disorder with agoraphobia: a two-year follow-up. J Anxiety Disord 2009, 23:1139-1147.

149. Roberge P, Marchand A, Reinharz D, Savard P: Cognitive-behavioral treatment for panic disorder with agoraphobia: a randomized, controlled trial and cost-effectiveness analysis. Behav Modif 2008, 32:333-351.

150. Meyerbroker K, Emmelkamp PM: Virtual reality exposure therapy in anxiety disorders: a systematic review of process-and-outcome studies. Depress Anxiety 2010, 27:933-944.

151. Botella C, García-Palacios A, Villa H, Baños RM, Quero S, Alcañiz M, Riva G Virtual reality exposure in the treatment of panic disorder and agoraphobia: A controlled study. Clin Psychol Psychother 2007, 14:164-175.

152. Malbos E, Rapee RM, Kavakli M: Isolating the effect of virtual reality based exposure therapy for agoraphobia: a comparative trial. Stud Health Technol Inform 2011, 167:45-50.

153. Perez-Ara MA, Quero S, Botella C, Banos R, Andreu-Mateu S, GarciaPalacios A, Breton-Lopez J: Virtual reality interoceptive exposure for the treatment of panic disorder and agoraphobia. Stud Health Technol Inform 2010, 154:77-81.

154. Vincelli F, Anolli L, Bouchard S, Wiederhold BK, Zurloni V, Riva G: Experiential cognitive therapy in the treatment of panic disorders with agoraphobia: a controlled study. Cyberpsychol Behav 2003, 6:321-328.

155. Hecker J, Losee M, Fritzler B, Fink C: Self-directed versus therapistdirected cognitive behavioural treatment for panic disorder. J Anxiety Disord 1996, 10:253-265.

156. Lidren DM, Watkins PL, Gould RA, Clum GA, Asterino M, Tulloch HL: A comparison of bibliotherapy and group therapy in the treatment of panic disorder. J Consult Clin Psychol 1994, 62:865-869.

157. Carlbring P, Maurin T, Sjomark J, Maurin L, Westling BE, Ekselius L, Cuijpers $\mathrm{P}$, Andersson $\mathrm{G}$ : All at once or one at a time? A randomized controlled trial comparing two ways to deliver bibliotherapy for panic disorder. Cogn Behav Ther 2011, 40:228-235.

158. Nordin S, Carlbring P, Cuijpers P, Andersson G: Expanding the limits of bibliotherapy for panic disorder: randomized trial of self-help without support but with a clear deadline. Behav Ther 2010, 41:267-276.

159. McNamee G, O'Sullivan G, Lelliott P, Marks I: Telephone-guided treatment for housebound agoraphobics with panic disorder: exposure versus relaxation. Behav Ther 1989, 20:491-497.

160. Swinson RP, Fergus KD, Cox BJ, Wickwire K: Efficacy of telephoneadministered behavioral therapy for panic disorder with agoraphobia. Behav Res Ther 1995, 33:465-469.

161. Bouchard S, Paquin B, Payeur R, Allard M, Rivard V, Fournier T, Renaud P, Lapierre J: Delivering cognitive-behavior therapy for panic disorder with agoraphobia in videoconference. Telemed J E Health 2004, 10:13-25.

162. Carlbring P, Ekselius L, Andersson G: Treatment of panic disorder via the Internet: a randomized trial of CBT vs. applied relaxation. $J$ Behav Ther Exp Psychiatry 2003, 34:129-140.

163. Newman MG, Kenardy J, Herman S, Taylor CB: Comparison of palmtopcomputer-assisted brief cognitive-behavioral treatment to cognitivebehavioral treatment for panic disorder. J Consult Clin Psychol 1997, 65:178-183.

164. Bergstrom J, Andersson G, Ljotsson B, Ruck C, Andreewitch S, Karlsson A, Carlbring $P$, Andersson $E$, Lindefors $N$ : Internet-versus group-administered cognitive behaviour therapy for panic disorder in a psychiatric setting: a randomised trial. BMC Psychiatry 2010, 10:54

165. Carlbring P, Bohman S, Brunt S, Buhrman M, Westling BE, Ekselius $L$, Andersson G: Remote treatment of panic disorder: a randomized trial of internet-based cognitive behavior therapy supplemented with telephone calls. Am J Psychiatry 2006, 163:2119-2125.

166. Kiropoulos LA, Klein B, Austin DW, Gilson K, Pier C, Mitchell J, Ciechomski L: Is internet-based CBT for panic disorder and agoraphobia as effective as face-to-face CBT? J Anxiety Disord 2008, 22:1273-1284.

167. Wims E, Titov N, Andrews G, Choi I: Clinician-assisted Internet-based treatment is effective for panic: a randomized controlled trial. Aust $N Z$ J Psychiatry 2010, 44:599-607.

168. Klein B, Austin D, Pier C, Kiropoulos L, Shandley K, Mitchell J, Gilson K, Ciechomski L: Internet-based treatment for panic disorder: does frequency of therapist contact make a difference? Cogn Behav Ther 2009, 38:100-113.

169. Ruwaard J, Broeksteeg J, Schrieken B, Emmelkamp P, Lange A: Web-based therapist-assisted cognitive behavioral treatment of panic symptoms: a randomized controlled trial with a three-year follow-up. J Anxiety Disord 2010, 24:387-396. 
170. Clark DM, Salkovskis PM, Hackmann A, Wells A, Ludgate J, Gelder M: Brief cognitive therapy for panic disorder: a randomized controlled trial. J Consult Clin Psychol 1999, 67:583-589.

171. Bohni MK, Spindler H, Arendt M, Hougaard E, Rosenberg NK: A randomized study of massed three-week cognitive behavioural therapy schedule for panic disorder. Acta Psychiatr Scand 2009, 120:187-195.

172. Dow MG, Kenardy JA, Johnston DW, Newman MG, Taylor CB, Thomson A: Prognostic indices with brief and standard CBT for panic disorder: II. Moderators of outcome. Psychol Med 2007, 37:1503-1509.

173. Craske MG, Roy-Byrne P, Stein MB, Sullivan G, Hazlett-Stevens H, Bystritsky A, Sherbourne C: CBT intensity and outcome for panic disorder in a primary care setting. Behav Ther 2006, 37:112-119.

174. Haby MM, Donnelly M, Corry J, Vos T: Cognitive behavioural therapy for depression, panic disorder and generalized anxiety disorder: a metaregression of factors that may predict outcome. Aust N Z J Psychiatry 2006, 40:9-19.

175. Dow MG, Kenardy JA, Johnston DW, Newman MG, Taylor CB, Thomson A: Prognostic indices with brief and standard CBT for panic disorder: I. Predictors of outcome. Psychol Med 2007, 37:1493-1502.

176. Bouchard S, Gauthier J, Nouwen A, Ivers H, Vallieres A, Simard S, Fournier T: Temporal relationship between dysfunctional beliefs, selfefficacy and panic apprehension in the treatment of panic disorder with agoraphobia. J Behav Ther Exp Psychiatry 2007, 38:275-292.

177. Feske U, Goldstein AJ: Eye movement desensitization and reprocessing treatment for panic disorder: a controlled outcome and partial dismantling study. J Consult Clin Psychol 1997, 65:1026-1035.

178. Goldstein AJ, de Beurs E, Chambless DL, Wilson KA: EMDR for panic disorder with agoraphobia: comparison with waiting list and credible attention-placebo control conditions. J Consult Clin Psychol 2000, 68:947-956

179. Furukawa TA, Watanabe N, Churchill R: Psychotherapy plus antidepressant for panic disorder with or without agoraphobia: systematic review. Br J Psychiatry 2006, 188:305-312.

180. Furukawa TA, Watanabe N, Churchill R: Combined psychotherapy plus antidepressants for panic disorder with or without agoraphobia. Cochrane Database Syst Rev 2007, CD004364.

181. Mitte K: A meta-analysis of the efficacy of psycho- and pharmacotherapy in panic disorder with and without agoraphobia. $J$ Affect Disord 2005, 88:27-45.

182. Watanabe N, Churchill R, Furukawa TA: Combined psychotherapy plus benzodiazepines for panic disorder. Cochrane Database Syst Rev 2009, CD005335.

183. Koszycki D, Taljaard M, Segal Z, Bradwejn J: A randomized trial of sertraline, self-administered cognitive behavior therapy, and their combination for panic disorder. Psychol Med 2011, 41:373-383.

184. Kim YW, Lee SH, Choi TK, Suh SY, Kim B, Kim CM, Cho SJ, Kim MJ, Yook K, Ryu $M$, et al: Effectiveness of mindfulness-based cognitive therapy as an adjuvant to pharmacotherapy in patients with panic disorder or generalized anxiety disorder. Depress Anxiety 2009, 26:601-606.

185. Kim B, Lee SH, Kim YW, Choi TK, Yook K, Suh SY, Cho SJ, Yook KH: Effectiveness of a mindfulness-based cognitive therapy program as an adjunct to pharmacotherapy in patients with panic disorder. J Anxiety Disord 2010, 24:590-595.

186. Schmidt NB, Wollaway-Bickel K, Trakowski JH, Santiago HT, Vasey M: Antidepressant discontinuation in the context of cognitive behavioral treatment for panic disorder. Behav Res Ther 2002, 40:67-73.

187. Otto MW, McHugh RK, Simon NM, Farach FJ, Worthington JJ, Pollack MH: Efficacy of CBT for benzodiazepine discontinuation in patients with panic disorder: further evaluation. Behav Res Ther 2010, 48:720-727.

188. Bruce T, Spiegel D, Hegel M: Cognitive-behavioral therapy helps prevent relapse and recurrence of panic disorder following alprazolam discontinuation: a long-term follow-up of the Peoria and Dartmouth studies. J Consult Clin Psychol 1999, 67:151-156.

189. Katon W, Russo J, Sherbourne C, Stein MB, Craske M, Fan MY, Roy-Byrne P: Incremental cost-effectiveness of a collaborative care intervention for panic disorder. Psychol Med 2006, 36:353-363.

190. Cottraux J, Note ID, Cungi C, Legeron P, Heim F, Chneiweiss L, Bernard G, Bouvard M: A controlled study of cognitive behaviour therapy with buspirone or placebo in panic disorder with agoraphobia. Br J Psychiatry 1995, 167:635-641.
191. Otto MW, Tolin DF, Simon NM, Pearlson GD, Basden S, Meunier SA, Hofmann SG, Eisenmenger K, Krystal JH, Pollack MH: Efficacy of dcycloserine for enhancing response to cognitive-behavior therapy for panic disorder. Biol Psychiatry 2010, 67:365-370.

192. Siegmund A, Golfels F, Finck C, Halisch A, Rath D, Plag J, Strohle A: Dcycloserine does not improve but might slightly speed up the outcome of in-vivo exposure therapy in patients with severe agoraphobia and panic disorder in a randomized double blind clinical trial. J Psychiatr Res 2011, 45:1042-1047.

193. Nations KR, Smits JA, Tolin DF, Rothbaum BO, Hofmann SG, Tart CD, Lee A, Schipper J, Sjogren M, Xue D, et al: Evaluation of the glycine transporter inhibitor Org 25935 as augmentation to cognitive-behavioral therapy for panic disorder: a multicenter, randomized, double-blind, placebocontrolled trial. J Clin Psychiatry 2012, 73:647-653.

194. Fava GA, Rafanelli C, Grandi S, Conti S, Ruini C, Mangelli L, Belluardo P: Long-term outcome of panic disorder with agoraphobia treated by exposure. Psychol Med 2001, 31:891-898.

195. Bakker A, van Balkom A, Spinhoven P: SSRls vs. TCAs in the treatment of panic disorder: a meta-analysis. Acta Psychiatr Scand 2002, 106:163-167.

196. Otto MW, Tuby KS, Gould RA, McLean RY, Pollack MH: An effect-size analysis of the relative efficacy and tolerability of serotonin selective reuptake inhibitors for panic disorder. Am J Psychiatry 2001, 158:1989-1992.

197. Andrisano C, Chiesa A, Serretti A: Newer antidepressants and panic disorder: a meta-analysis. Int Clin Psychopharmacol 2013, 28:33-45.

198. Stahl SM, Gergel I, Li D: Escitalopram in the treatment of panic disorder: a randomized, double-blind, placebo-controlled trial. J Clin Psychiatry 2003, 64:1322-1327.

199. Wade AG, Lepola U, Koponen HJ, Pedersen V, Pedersen T: The effect of citalopram in panic disorder. Br J Psychiatry 1997, 170:549-553.

200. Seedat S, van Rheede van Oudtshoorn E, Muller JE, Mohr N, Stein DJ: Reboxetine and citalopram in panic disorder: a single-blind, cross-over, flexible-dose pilot study. Int Clin Psychopharmacol 2003, 18:279-284.

201. Michelson D, Lydiard RB, Pollack MH, Tamura RN, Hoog SL, Tepner R, Demitrack MA, Tollefson GD: Outcome assessment and clinical improvement in panic disorder: evidence from a randomized controlled trial of fluoxetine and placebo. The Fluoxetine Panic Disorder Study Group. Am J Psychiatry 1998, 155:1570-1577.

202. Michelson D, Allgulander C, Dantendorfer K, Knezevic A, Maierhofer D, Micev V, Paunovic V, Timotijevic I, Sarkar N, Skoglund L, Pemberton S: Efficacy of usual antidepressant dosing regimens of fluoxetine in panic disorder: randomised, placebo-controlled trial. Br J Psychiatry 2001, 179:514-518.

203. Ribeiro L, Busnello J, Kauer-Sant'Anna M, Madruga M, Quevedo J, Busnello E, Kapczinski F: Mirtazapine versus fluoxetine in the treatment of panic disorder. Braz J Med Biol Res 2001, 34:1303-1307.

204. Tiller JW, Bouwer C, Behnke K: Moclobemide and fluoxetine for panic disorder. International Panic Disorder Study Group. Eur Arch Psychiatry Clin Neurosci 1999, 249(Suppl 1):S7-10.

205. Black DW, Wesner R, Bowers W, Gabel J: A comparison of fluvoxamine, cognitive therapy, and placebo in the treatment of panic disorder. Arch Gen Psychiatry 1993, 50:44-50.

206. Asnis G, Hameedi F, Goddard A, Potkin S, Black D, Jameel M, Desagani K, Woods S: Fluvoxamine in the treatment of panic disorder: a multicenter, double-blind, placebo-controlled study in outpatients. Psychiatry Res 2001, 103:1-14.

207. Bakish D, Hooper CL, Filteau MJ, Charbonneau Y, Fraser G, West DL, Thibaudeau C, Raine D: A double-blind placebo-controlled trial comparing fluvoxamine and imipramine in the treatment of panic disorder with or without agoraphobia. Psychopharmacol Bull 1996, 32:135-141.

208. Den Boer JA, Westenberg HG: Serotonin function in panic disorder: a double blind placebo controlled study with fluvoxamine and ritanserin. Psychopharmacology (Berl) 1990, 102:85-94.

209. Hoehn-Saric R, McLeod DR, Hipsley PA: Effect of fluvoxamine on panic disorder. J Clin Psychopharmacol 1993, 13:321-326.

210. Sharp DM, Power KG, Simpson RJ, Swanson V, Anstee JA: Global measures of outcome in a controlled comparison of pharmacological and psychological treatment of panic disorder and agoraphobia in primary care. Br J Gen Pract 1997, 47:150-155. 
211. Bakker A, van Dyck R, Spinhoven P, van Balkom AJ: Paroxetine, clomipramine, and cognitive therapy in the treatment of panic disorder. J Clin Psychiatry 1999, 60:831-838.

212. Ballenger JC, Wheadon DE, Steiner M, Bushnell W, Gergel IP: Double-blind, fixed-dose, placebo-controlled study of paroxetine in the treatment of panic disorder. Am J Psychiatry 1998, 155:36-42

213. Lecrubier $Y$, Bakker A, Dunbar G, Judge R: A comparison of paroxetine, clomipramine and placebo in the treatment of panic disorder. Collaborative Paroxetine Panic Study Investigators. Acta Psychiatr Scand 1997, 95:145-152

214. Oehrberg S, Christiansen PE, Behnke K, Borup AL, Severin B, Soegaard J, Calberg $H$, Judge R, Ohrstrom JK, Manniche PM: Paroxetine in the treatment of panic disorder. A randomised, double-blind, placebocontrolled study. Br J Psychiatry 1995, 167:374-379.

215. Pollack M, Mangano R, Entsuah R, Tzanis E, Simon NM, Zhang Y: A randomized controlled trial of venlafaxine ER and paroxetine in the treatment of outpatients with panic disorder. Psychopharmacology (Berl) 2007, 194:233-242.

216. Pollack MH, Lepola U, Koponen H, Simon NM, Worthington JJ, Emilien G, Tzanis E, Salinas E, Whitaker T, Gao B: A double-blind study of the efficacy of venlafaxine extended-release, paroxetine, and placebo in the treatment of panic disorder. Depress Anxiety 2007, 24:1-14.

217. Prosser JM, Yard S, Steele A, Cohen LJ, Galynker II: A comparison of lowdose risperidone to paroxetine in the treatment of panic attacks: a randomized, single-blind study. BMC Psychiatry 2009, 9:25.

218. Nardi AE, Valenca AM, Freire RC, Mochcovitch MD, Amrein R, Sardinha A, Levitan MN, Nascimento I, de-Melo-Neto VL, King AL, et al: Psychopharmacotherapy of panic disorder: 8-week randomized trial with clonazepam and paroxetine. Braz J Med Biol Res 2011, 44:366-373.

219. Bertani A, Perna G, Migliarese G, Di Pasquale D, Cucchi M, Caldirola D, Bellodi $L$ : Comparison of the treatment with paroxetine and reboxetine in panic disorder: a randomized, single-blind study. Pharmacopsychiatry 2004, 37:206-210.

220. Londborg PD, Wolkow R, Smith WT, DuBoff E, England D, Ferguson J, Rosenthal $M$, Weise C: Sertraline in the treatment of panic disorder. A multi-site, double-blind, placebo-controlled, fixed-dose investigation. $\mathrm{Br}$ J Psychiatry 1998, 173:54-60.

221. Pohl RB, Wolkow RM, Clary CM: Sertraline in the treatment of panic disorder: a double-blind multicenter trial. Am J Psychiatry 1998, 155:1189-1195.

222. Pollack MH, Otto MW, Worthington JJ, Manfro GG, Wolkow R: Sertraline in the treatment of panic disorder: a flexible-dose multicenter trial. Arch Gen Psychiatry 1998, 55:1010-1016.

223. Pollack M, Rapaport M, Clary C, Mardekian J, Wolkow R: Sertraline treatment of panic disorder: response in patients at risk for poor outcome. J Clin Psychiatry 2000, 61:922-927.

224. Lepola U, Arato M, Zhu Y, Austin C: Sertraline versus imipramine treatment of comorbid panic disorder and major depressive disorder. J Clin Psychiatry 2003, 64:654-662.

225. Sheehan DV, Burnham DB, lyengar MK, Perera P: Efficacy and tolerability of controlled-release paroxetine in the treatment of panic disorder. J Clin Psychiatry 2005, 66:34-40.

226. Perna G, Dacco S, Menotti R, Caldirola D: Antianxiety medications for the treatment of complex agoraphobia: pharmacological interventions for a behavioral condition. Neuropsychiatr Dis Treat 2011, 7:621-637.

227. Pollack MH, Worthington JJ 3rd, Otto MW, Maki KM, Smoller JW, Manfro GG, Rudolph R, Rosenbaum JF: Venlafaxine for panic disorder: results from a double-blind, placebo-controlled study. Psychopharmacol Bull 1996, 32:667-670.

228. Bradwejn J, Ahokas A, Stein DJ, Salinas E, Emilien G, Whitaker T: Venlafaxine extended-release capsules in panic disorder: Flexibledose, double-blind, placebo-controlled study. Br J Psychiatry 2005, 187:352-359.

229. Liebowitz MR, Asnis G, Mangano R, Tzanis E: A double-blind, placebocontrolled, parallel-group, flexible-dose study of venlafaxine extended release capsules in adult outpatients with panic disorder. J Clin Psychiatry 2009, 70:550-561.

230. Simon NM, Kaufman RE, Hoge EA, Worthington JJ, Herlands NN, Owens ME, Pollack MH: Open-label support for duloxetine for the treatment of panic disorder. CNS Neurosci Ther 2009, 15:19-23.
231. Blaya C, Seganfredo AC, Dornelles M, Torres M, Paludo A, Heldt E, Manfro GG: The efficacy of milnacipran in panic disorder: an open trial. Int Clin Psychopharmacol 2007, 22:153-158.

232. Kruger MB, Dahl AA: The efficacy and safety of moclobemide compared to clomipramine in the treatment of panic disorder. Eur Arch Psychiatry Clin Neurosci 1999, 249(Suppl 1):S19-24.

233. Modigh K, Westberg P, Eriksson E: Superiority of clomipramine over imipramine in the treatment of panic disorder: a placebo-controlled trial. J Clin Psychopharmacol 1992, 12:251-261.

234. Cox BJ, Endler NS, Lee PS, Swinson RP: A meta-analysis of treatments for panic disorder with agoraphobia: imipramine, alprazolam, and in vivo exposure. J Behav Ther Exp Psychiatry 1992, 23:175-182.

235. Drug treatment of panic disorder. Comparative efficacy of alprazolam, imipramine, and placebo. Cross-National Collaborative Panic Study, Second Phase Investigators. Br J Psychiatry 1992, 160:191-202, discussion 202-195.

236. Andersch S, Rosenberg NK, Kullingsjo H, Ottosson JO, Bech P, BruunHansen J, Hanson L, Lorentzen K, Mellergard M, Rasmussen S, et al: Efficacy and safety of alprazolam, imipramine and placebo in treating panic disorder. A Scandinavian multicenter study. Acta Psychiatr Scand Supp/ 1991, 365:18-27.

237. Taylor CB, Hayward C, King R, Ehlers A, Margraf J, Maddock R, Clark D, Roth WT Agras WS: Cardiovascular and symptomatic reduction effects of alprazolam and imipramine in patients with panic disorder: results of a double-blind, placebo-controlled trial. J Clin Psychopharmacol 1990, 10:112-118.

238. Barlow D, Gorman J, Shear M, Woods S: Cognitive-behavioral therapy, imipramine, or their combination for panic disorder: A randomized controlled trial. JAMA 2000, 283:2529-2536.

239. Marchand A, Coutu MF, Dupuis G, Fleet R, Borgeat F, Todorov C, Mainguy N: Treatment of panic disorder with agoraphobia: randomized placebo-controlled trial of four psychosocial treatments combined with imipramine or placebo. Cogn Behav Ther 2008, 37:146-159.

240. Sheehan DV, Ballenger J, Jacobsen G: Treatment of endogenous anxiety with phobic, hysterical, and hypochondriacal symptoms. Arch Gen Psychiatry 1980, 37:51-59.

241. Uhlenhuth EH, Warner TD, Matuzas W: Interactive model of therapeutic response in panic disorder: moclobemide, a case in point. $J$ Clin Psychopharmacol 2002, 22:275-284.

242. Loerch B, Graf-Morgenstern M, Hautzinger M, Schlegel S, Hain C, Sandmann J, Benkert O: Randomised placebo-controlled trial of moclobemide, cognitive-behavioural therapy and their combination in panic disorder with agoraphobia. Br J Psychiatry 1999, 174:205-212.

243. Nardi AE, Lopes FL, Valenca AM, Freire RC, Nascimento I, Veras AB, Mezzasalma MA, de-Melo-Neto VL, Soares-Filho GL, King AL, et al: Doubleblind comparison of 30 and $60 \mathrm{mg}$ tranylcypromine daily in patients with panic disorder comorbid with social anxiety disorder. Psychiatry Res 2010, 175:260-265.

244. Versiani M, Cassano G, Perugi G, Benedetti A, Mastalli L, Nardi A, Savino M: Reboxetine, a selective norepinephrine reuptake inhibitor, is an effective and well-tolerated treatment for panic disorder. J Clin Psychiatry 2002, 63:31-37.

245. Boshuisen M, Slaap B, Vester-Blokland E, den Boer J: The effect of mirtazapine in panic disorder: an open label pilot study with a singleblind placebo run-in period. Int Clin Psychopharmacol 2001, 16:363-368.

246. Sarchiapone M, Amore M, De Risio S, Carli V, Faia V, Poterzio F, Balista C, Camardese G, Ferrari G: Mirtazapine in the treatment of panic disorder: an open-label trial. Int Clin Psychopharmacol 2003, 18:35-38.

247. Sheehan DV, Davidson J, Manschreck T, Van Wyck Fleet J: Lack of efficacy of a new antidepressant (bupropion) in the treatment of panic disorder with phobias. J Clin Psychopharmacol 1983, 3:28-31.

248. Simon NM, Emmanuel N, Ballenger J, Worthington JJ, Kinrys G, Korbly NB, Farach FJ, Pollack MH: Bupropion sustained release for panic disorder. Psychopharmacol Bull 2003, 37:66-72.

249. Boyer W: Serotonin uptake inhibitors are superior to imipramine and alprazolam in alleviating panic attacks: a meta-analysis. Int Clin Psychopharmacol 1995, 10:45-49.

250. Tesar GE, Rosenbaum JF, Pollack MH, Otto MW, Sachs GS, Herman JB, Cohen LS, Spier SA: Double-blind, placebo-controlled comparison of clonazepam and alprazolam for panic disorder. J Clin Psychiatry 1991, 52:69-76. 
251. Schweizer E, Pohl R, Balon R, Fox I, Rickels K, Yeragani VK: Lorazepam vs. alprazolam in the treatment of panic disorder. Pharmacopsychiatry 1990, 23:90-93.

252. Moylan S, Staples J, Ward SA, Rogerson J, Stein DJ, Berk M: The efficacy and safety of alprazolam versus other benzodiazepines in the treatment of panic disorder. J Clin Psychopharmacol 2011, 31:647-652

253. Marquez M, Arenoso H, Caruso N: Efficacy of alprazolam sublingual tablets in the treatment of the acute phase of panic disorders. Actas Esp Psiquiatr 2011, 39:88-94.

254. Sheehan D, Raj A, Harnett-Sheehan K, Soto S, Knapp E: The relative efficacy of high-dose buspirone and alprazolam in the treatment of panic disorder: a double-blind placebo-controlled study. Acta Psychiatr Scand 1993, 88:1-11.

255. Beauclair L, Fontaine R, Annable L, Holobow N, Chouinard G Clonazepam in the treatment of panic disorder: a double-blind, placebo-controlled trial investigating the correlation between clonazepam concentrations in plasma and clinical response. J Clin Psychopharmacol 1994, 14:111-118.

256. Moroz G, Rosenbaum JF: Efficacy, safety, and gradual discontinuation of clonazepam in panic disorder: a placebo-controlled, multicenter study using optimized dosages. J Clin Psychiatry 1999, 60:604-612.

257. Rosenbaum JF, Moroz G, Bowden CL: Clonazepam in the treatment of panic disorder with or without agoraphobia: a dose-response study of efficacy, safety, and discontinuance. Clonazepam Panic Disorder DoseResponse Study Group. J Clin Psychopharmacol 1997, 17:390-400.

258. Valenca A, Nardi A, Nascimento I, Mezzasalma M, Lopes F, Zin W: Doubleblind clonazepam vs placebo in panic disorder treatment. Arq Neuropsiquiatr 2000, 58:1025-1029.

259. Charney DS, Woods SW: Benzodiazepine treatment of panic disorder: a comparison of alprazolam and lorazepam. J Clin Psychiatry 1989, 50:418-423.

260. de Jonghe F, Swinkels J, Tuynman-Qua H, Jonkers F: A comparative study of suriclone, lorazepam and placebo in anxiety disorder. Pharmacopsychiatry 1989, 22:266-271.

261. Dunner DL, Ishiki D, Avery DH, Wilson LG, Hyde TS: Effect of alprazolam and diazepam on anxiety and panic attacks in panic disorder: a controlled study. J Clin Psychiatry 1986, 47:458-460.

262. Noyes R Jr., Anderson DJ, Clancy J, Crowe RR, Slymen DJ, Ghoneim MM, Hinrichs JV: Diazepam and propranolol in panic disorder and agoraphobia. Arch Gen Psychiatry 1984, 41:287-292.

263. Noyes R Jr., Burrows GD, Reich JH, Judd FK, Garvey MJ, Norman TR, Cook BL, Marriott P: Diazepam versus alprazolam for the treatment of panic disorder. J Clin Psychiatry 1996, 57:349-355.

264. Pollack MH, Simon NM, Worthington JJ, Doyle AL, Peters P, Toshkov F, Otto MW: Combined paroxetine and clonazepam treatment strategies compared to paroxetine monotherapy for panic disorder. $J$ Psychopharmacol 2003, 17:276-282.

265. Goddard AW, Brouette T, Almai A, Jetty P, Woods SW, Charney D: Early coadministration of clonazepam with sertraline for panic disorder. Arch Gen Psychiatry 2001, 58:681-686.

266. Katzelnick DJ, Saidi J, Vanelli MR, Jefferson JW, Harper JM, McCrary KE: Time to response in panic disorder in a naturalistic setting: combination therapy with alprazolam orally disintegrating tablets and serotonin reuptake inhibitors compared to serotonin reuptake inhibitors alone. Psychiatry (Edgmont) 2006, 3:39-49.

267. Galynker I, Khan A, Grebchenko Y, Ten A, Malaya L, Yanowitch P, Cohen L: Low-dose risperidone and quetiapine as monotherapy for comorbid anxiety and depression [Letter]. J Clin Psychiatry 2005, 66:544.

268. Hollifield M, Thompson PM, Ruiz JE, Uhlenhuth EH: Potential effectiveness and safety of olanzapine in refractory panic disorder. Depress Anxiety 2005, 21:33-40

269. Hoge EA, Worthington JJ 3rd, Kaufman RE, Delong HR, Pollack MH, Simon NM: Aripiprazole as augmentation treatment of refractory generalized anxiety disorder and panic disorder. CNS Spectr 2008 13:522-527.

270. Sepede G, De Berardis D, Gambi F, Campanella D, La Rovere R, D'Amico M, Cicconetti A, Penna L, Peca S, Carano A, et al: Olanzapine augmentation in treatment-resistant panic disorder: a 12-week, fixed-dose, open-label trial. J Clin Psychopharmacol 2006, 26:45-49.

271. Simon NM, Hoge EA, Fischmann D, Worthington JJ, Christian KM, Kinrys G, Pollack $\mathrm{MH}$ : An open-label trial of risperidone augmentation for refractory anxiety disorders. J Clin Psychiatry 2006, 67:381-385.
272. Baetz M, Bowen RC: Efficacy of divalproex sodium in patients with panic disorder and mood instability who have not responded to conventional therapy. Can J Psychiatry 1998, 43:73-77.

273. Keck PE Jr., Taylor VE, Tugrul KC, McElroy SL, Bennett JA: Valproate treatment of panic disorder and lactate-induced panic attacks. Biol Psychiatry 1993, 33:542-546.

274. Woodman $\mathrm{CL}$, Noyes $\mathrm{R}$ Jr.: Panic disorder: treatment with valproate J Clin Psychiatry 1994, 55:134-136.

275. Primeau F, Fontaine R, Beauclair L: Valproic acid and panic disorder. Can J Psychiatry 1990, 35:248-250.

276. Papp LA: Safety and efficacy of levetiracetam for patients with panic disorder: results of an open-label, fixed-flexible dose study. J Clin Psychiatry 2006, 67:1573-1576.

277. Pande AC, Pollack MH, Crockatt J, Greiner M, Chouinard G, Lydiard RB, Taylor CB, Dager SR, Shiovitz T: Placebo-controlled study of gabapentin treatment of panic disorder. J Clin Psychopharmacol 2000, 20:467-471

278. Zwanzger P, Eser D, Nothdurfter C, Baghai TC, Moller HJ, Padberg F, Rupprecht R: Effects of the GABA-reuptake inhibitor tiagabine on panic and anxiety in patients with panic disorder. Pharmacopsychiatry 2009, 42:266-269.

279. Sheehan DV, Sheehan KH, Raj BA, Janavs J: An open-label study of tiagabine in panic disorder. Psychopharmacol Bull 2007, 40:32-40.

280. Uhde TW, Stein MB, Post RM: Lack of efficacy of carbamazepine in the treatment of panic disorder. Am J Psychiatry 1988, 145:1104-1109.

281. Perugi G, Frare F, Toni C, Tusini G, Vannucchi G, Akiskal HS: Adjunctive valproate in panic disorder patients with comorbid bipolar disorder or otherwise resistant to standard antidepressants: a 3-year "open" followup study. Eur Arch Psychiatry Clin Neurosci 2010, 260:553-560

282. Sheehan DV, Raj AB, Sheehan KH, Soto S: Is buspirone effective for panic disorder? J Clin Psychopharmacol 1990, 10:3-11.

283. Charney DS, Woods SW, Goodman WK, Rifkin B, Kinch M, Aiken B, Quadrino LM, Heninger GR: Drug treatment of panic disorder: the comparative efficacy of imipramine, alprazolam, and trazodone. J Clin Psychiatry 1986, 47:580-586.

284. Munjack DJ, Crocker B, Cabe D, Brown R, Usigli R, Zulueta A, McManus M, McDowell D, Palmer R, Leonard M: Alprazolam, propranolol, and placebo in the treatment of panic disorder and agoraphobia with panic attacks. J Clin Psychopharmacol 1989, 9:22-27.

285. Ravaris CL, Friedman MJ, Hauri PJ, McHugo GJ: A controlled study of alprazolam and propranolol in panic-disordered and agoraphobic outpatients. J Clin Psychopharmacol 1991, 11:344-350.

286. Hirschmann S, Dannon PN, lancu I, Dolberg OT, Zohar J, Grunhaus L: Pindolol augmentation in patients with treatment-resistant panic disorder: A double-blind, placebo-controlled trial. J Clin Psychopharmacol 2000, 20:556-559.

287. Lepola UM, Wade AG, Leinonen EV, Koponen HJ, Frazer J, Sjodin I, Penttinen JT, Pedersen T, Lehto HJ: A controlled, prospective, 1-year trial of citalopram in the treatment of panic disorder. J Clin Psychiatry 1998, 59:528-534.

288. Dannon PN, lancu I, Lowengrub K, Gonopolsky Y, Musin E, Grunhaus L, Kotler M: A naturalistic long-term comparison study of selective serotonin reuptake inhibitors in the treatment of panic disorder. Clin Neuropharmacol 2007, 30:326-334.

289. Lecrubier $Y$, Judge $R$ : Long-term evaluation of paroxetine, clomipramine and placebo in panic disorder. Collaborative Paroxetine Panic Study Investigators. Acta Psychiatr Scand 1997, 95:153-160.

290. Nardi AE, Freire RC, Mochcovitch MD, Amrein R, Levitan MN, King AL, Valenca $A M$, Veras $A B$, Paes $F$, Sardinha $A$, et al: A randomized, naturalistic, parallel-group study for the long-term treatment of panic disorder with clonazepam or paroxetine. J Clin Psychopharmacol 2012, 32:120-126.

291. Curtis GC, Massana J, Udina C, Ayuso JL, Cassano GB, Perugi G: Maintenance drug therapy of panic disorder. J Psychiatr Res 1993, 27(Suppl 1):127-142.

292. Mavissakalian M, Perel JM: Clinical experiments in maintenance and discontinuation of imipramine therapy in panic disorder with agoraphobia. Arch Gen Psychiatry 1992, 49:318-323.

293. Rickels K, Schweizer E: Panic disorder: long-term pharmacotherapy and discontinuation. J Clin Psychopharmacol 1998, 18:12S-18S.

294. Ferguson JM, Khan A, Mangano R, Entsuah R, Tzanis E: Relapse prevention of panic disorder in adult outpatient responders to treatment with venlafaxine extended release. J Clin Psychiatry 2007, 68:58-68. 
295. Mavissakalian MR, Perel JM: Long-term maintenance and discontinuation of imipramine therapy in panic disorder with agoraphobia. Arch Gen Psychiatry 1999, 56:821-827.

296. Nardi $A E$, Freire $R C$, Valenca AM, Amrein $R$, de Cerqueira AC, Lopes $F L$, Nascimento I, Mezzasalma MA, Veras AB, Sardinha A, et al: Tapering clonazepam in patients with panic disorder after at least 3 years of treatment. J Clin Psychopharmacol 2010, 30:290-293.

297. Mannu P, Rinaldi S, Fontani V, Castagna A, Margotti ML: Noninvasive brain stimulation by radioelectric asymmetric conveyor in the treatment of agoraphobia: open-label, naturalistic study. Patient Prefer Adherence 2011, 5:575-580.

298. Mannu P, Rinaldi S, Fontani V, Castagna A, Margotti ML: Radio electric treatment vs. escitalopram in the treatment of panic disorders associated with major depression: an open-label, naturalistic study. Acupunct Electrother Res 2009, 34:135-149.

299. Mantovani A, Lisanby SH, Pieraccini F, Ulivelli M, Castrogiovanni P, Rossi S: Repetitive transcranial magnetic stimulation (rTMS) in the treatment of panic disorder (PD) with comorbid major depression. J Affect Disord 2007, 102:277-280.

300. Prasko J, Zalesky R, Bares M, Horacek J, Kopecek M, Novak T, Paskova B: The effect of repetitive transcranial magnetic stimulation (rTMS) add on serotonin reuptake inhibitors in patients with panic disorder: a randomized, double blind sham controlled study. Neuro Endocrinol Lett 2007, 28:33-38

301. Meuret AE, Rosenfield D, Seidel A, Bhaskara L, Hofmann SG: Respiratory and cognitive mediators of treatment change in panic disorder: evidence for intervention specificity. J Consult Clin Psychol 2010, 78:691-704.

302. Wollburg E, Roth WT, Kim S: Effects of breathing training on voluntary hypo- and hyperventilation in patients with panic disorder and episodic anxiety. Appl Psychophysiol Biofeedback 2011, 36:81-91.

303. Wedekind D, Broocks A, Weiss N, Engel K, Neubert K, Bandelow B: A randomized, controlled trial of aerobic exercise in combination with paroxetine in the treatment of panic disorder. World I Biol Psychiatry 2010, 11:904-913.

304. Strohle A, Graetz B, Scheel M, Wittmann A, Feller C, Heinz A, Dimeo F: The acute antipanic and anxiolytic activity of aerobic exercise in patients with panic disorder and healthy control subjects. J Psychiatr Res 2009, 43:1013-1017.

305. Stinson FS, Dawson DA, Patricia Chou S, Smith S, Goldstein RB, June Ruan W, Grant BF: The epidemiology of DSM-IV specific phobia in the USA: results from the National Epidemiologic Survey on Alcohol and Related Conditions. Psychol Med 2007, 37:1047-1059.

306. LeBeau RT, Glenn D, Liao B, Wittchen HU, Beesdo-Baum K, Ollendick T, Craske MG: Specific phobia: a review of DSM-IV specific phobia and preliminary recommendations for DSM-V. Depress Anxiety 2010, 27:148-167.

307. Becker ES, Rinck M, Turke V, Kause P, Goodwin R, Neumer S, Margraf J: Epidemiology of specific phobia subtypes: findings from the Dresden Mental Health Study. Eur Psychiatry 2007, 22:69-74.

308. Benjet C, Borges G, Stein D, Mendez E, Medina-Mora M: Epidemiology of fears and specific phobia in adolescence: results from the Mexican Adolescent Mental Health Survey. J Clin Psychiatry 2012, 73:152-158.

309. Trumpf J, Margraf J, Vriends N, Meyer AH, Becker ES: Specific phobia predicts psychopathology in young women. Soc Psychiatry Psychiatr Epidemiol 2010, 45:1161-1166.

310. Choy Y, Fyer AJ, Goodwin RD: Specific phobia and comorbid depression: a closer look at the National Comorbidity Survey data. Compr Psychiatry 2007, 48:132-136

311. Hood H, Antony M: Evidence-based assessment and treatment of specific phobias in adults, Chapt.2. In Intensive one-session treatment of specific phobias. New York: Springer;Davis T, Ollendick T, Öst L-G 2012:19-42, Autism and Child Psychopathology Series.

312. Parsons TD, Rizzo AA: Affective outcomes of virtual reality exposure therapy for anxiety and specific phobias: a meta-analysis. J Behav Ther Exp Psychiatry 2008, 39:250-261.

313. Ollendick TH, Ost LG, Reuterskiold L, Costa N, Cederlund R, Sirbu C, Davis TE 3rd, Jarrett MA: One-session treatment of specific phobias in youth: a randomized clinical trial in the United States and Sweden. Consult Clin Psychol 2009, 77:504-516.

314. Craske MG, Kircanski K, Zelikowsky M, Mystkowski J, Chowdhury N, Baker A: Optimizing inhibitory learning during exposure therapy. Behav Res Ther 2008, 46:5-27.
315. Öst L-G, Fellenius J, Sterner U: Applied tension, exposure in vivo, and tension-only in the treatment of blood phobia. Behav Res Ther 1991, 29.561-574

316. Hellstrom K, Fellenius J, Öst L-G: One versus five sessions of applied tension in the treatment of blood phobia. Behav Res Ther 1996, 34:101-112.

317. Kettwich SC, Sibbitt WL Jr., Brandt JR, Johnson CR, Wong CS, Bankhurst AD: Needle phobia and stress-reducing medical devices in pediatric and adult chemotherapy patients. J Pediatr Oncol Nurs 2007 24:20-28

318. Vika M, Skaret E, Raadal M, Ost LG, Kvale G: One- vs. five-session treatment of intra-oral injection phobia: a randomized clinical study. Eur J Oral Sci 2009, 117:279-285

319. Krijn M, Emmelkamp PM, Olafsson RP, Bouwman M, van Gerwen LJ, Spinhoven P, Schuemie MJ, van der Mast CA: Fear of flying treatment methods: virtual reality exposure vs. cognitive behavioral therapy. Aviat Space Environ Med 2007, 78:121-128.

320. Van Gerwen L, Spinhoven P, Van Dyck R: Behavioral and cognitive group treatment for fear of flying: a randomized controlled trial. $J$ Behav Ther Exp Psychiatry 2006, 37:358-371.

321. Wiederhold BK, Jang DP, Gevirtz RG, Kim SI, Kim IY, Wiederhold MD: The treatment of fear of flying: a controlled study of imaginal and virtual reality graded exposure therapy. IEEE Trans Inf Technol Biomed 2002, 6:218-223.

322. Rothbaum BO, Hodges L, Smith S, Lee JH, Price L: A controlled study of virtual reality exposure therapy for the fear of flying. I Consult Clin Psychol 2000, 68:1020-1026.

323. Tortella-Feliu M, Botella C, Llabres J, Breton-Lopez JM, del Amo AR, Banos RM, Gelabert JM: Virtual reality versus computer-aided exposure treatments for fear of flying. Behav Modif 2011, 35:3-30

324. Rothbaum BO, Anderson P, Zimand E, Hodges L, Lang D, Wilson J: Virtual reality exposure therapy and standard (in vivo) exposure therapy in the treatment of fear of flying. Behav Ther 2006, 37:80-90.

325. Kim S, Palin F, Anderson P. Edwards S, Lindner G, Rothbaum BO: Use of skills learned in CBT for fear of flying: managing flying anxiety after September 11th. J Anxiety Disord 2008, 22:301-309.

326. Anderson P, Jacobs CH, Lindner GK, Edwards S, Zimand E, Hodges L, Rothbaum BO: Cognitive behavior therapy for fear of flying: sustainability of treatment gains after September 11. Behav Ther 2006, 37:91-97.

327. Emmelkamp PM, Krijn M, Hulsbosch AM, de Vries S, Schuemie MJ, van der Mast CA: Virtual reality treatment versus exposure in vivo: a comparative evaluation in acrophobia. Behav Res Ther 2002, 40:509-516.

328. Rothbaum BO, Hodges LF, Kooper R, Opdyke D, Williford JS, North M: Effectiveness of computer-generated (virtual reality) graded exposure in the treatment of acrophobia. Am J Psychiatry 1995, 152:626-628.

329. Krijn M, Emmelkamp PM, Olafsson RP, Schuemie MJ, van der Mast CA: Do self-statements enhance the effectiveness of virtual reality exposure therapy? A comparative evaluation in acrophobia. Cyberpsychol Behav 2007, 10:362-370.

330. Malbos E, Mestre DR, Note ID, Gellato C: Virtual reality and claustrophobia: multiple components therapy involving game editor virtual environments exposure. Cyberpsychol Behav 2008, 11:695-697.

331. Michaliszyn D, Marchand A, Bouchard S, Martel MO, Poirier-Bisson J. A randomized, controlled clinical trial of in virtuo and in vivo exposure for spider phobia. Cyberpsychol Behav Soc Netw 2010, 13:689-695.

332. Bouchard S, Cote S, St-Jacques J, Robillard G, Renaud P: Effectiveness of virtual reality exposure in the treatment of arachnophobia using $3 \mathrm{D}$ games. Technol Health Care 2006, 14:19-27.

333. Granado LC, Ranvaud R, Pelaez JR: A spiderless arachnophobia therapy: comparison between placebo and treatment groups and six-month follow-up study. Neural Plast 2007, 2007:10241

334. Andersson G, Waara J, Jonsson U, Malmaeus F, Carlbring P, Ost LG Internet-based self-help versus one-session exposure in the treatment of spider phobia: a randomized controlled trial. Cogn Behav Ther 2009, 38:114-120

335. Muller BH, Kull S, Wilhelm FH, Michael T: One-session computer-based exposure treatment for spider-fearful individuals-efficacy of a minimal self-help intervention in a randomised controlled trial. J Behav Ther Exp Psychiatry 2011, 42:179-184. 
336. Botella C, Quero S, Banos RM, Garcia-Palacios A, Breton-Lopez J, Alcaniz M, Fabregat S: Telepsychology and self-help: the treatment of phobias using the internet. Cyberpsychol Behav 2008, 11:659-664.

337. Botella C, Breton-Lopez J, Quero S, Banos R, Garcia-Palacios A: Treating cockroach phobia with augmented reality. Behav Ther 2010 41:401-413.

338. Ressler KJ, Rothbaum BO, Tannenbaum L, Anderson P, Graap K, Zimand E, Hodges L, Davis M: Cognitive enhancers as adjuncts to psychotherapy: use of D-cycloserine in phobic individuals to facilitate extinction of fear. Arch Gen Psychiatry 2004, 61:1136-1144.

339. Guastella AJ, Dadds MR, Lovibond PF, Mitchell P, Richardson R: A randomized controlled trial of the effect of $d$-cycloserine on exposure therapy for spider fear. J Psychiatr Res 2007, 41:466-471.

340. de Quervain DJ, Bentz D, Michael T, Bolt OC, Wiederhold BK, Margraf J, Wilhelm FH: Glucocorticoids enhance extinction-based psychotherapy. Proc Natl Acad Sci U S A 2011, 108:6621-6625.

341. Soravia LM, Heinrichs M, Aerni A, Maroni C, Schelling G, Ehlert U, Roozendaal B, de Quervain DJ: Glucocorticoids reduce phobic fear in humans. Proc Natl Acad Sci U S A 2006, 103:5585-5590.

342. Meyerbroeker K, Powers MB, van Stegeren A, Emmelkamp PM: Does yohimbine hydrochloride facilitate fear extinction in virtual reality treatment of fear of flying? A randomized placebo-controlled trial. Psychother Psychosom 2012, 81:29-37.

343. Powers MB, Smits JA, Otto MW, Sanders C, Emmelkamp PM: Facilitation of fear extinction in phobic participants with a novel cognitive enhancer: a randomized placebo controlled trial of yohimbine augmentation. J Anxiety Disord 2009, 23:350-356.

344. Kozak AT, Spates CR, McChargue DE, Bailey KC, Schneider KL, Liepman MR: Naltrexone renders one-session exposure therapy less effective: a controlled pilot study. J Anxiety Disord 2007, 21:142-152

345. Benjamin J, Ben-Zion I, Karbofsky E, Dannon P: Double-blind placebocontrolled pilot study of paroxetine for specific phobia. Psychopharmacology (Berl) 2000, 149:194-196.

346. Alamy S, Wei Z, Varia I, Davidson JR, Connor KM: Escitalopram in specific phobia: results of a placebo-controlled pilot trial. J Psychopharmacol 2008, 22:157-161.

347. Abene MV, Hamilton JD: Resolution of fear of flying with fluoxetine treatment. J Anxiety Disord 1998, 12:599-603.

348. Balon R: Fluvoxamine for phobia of storms. Acta Psychiatr Scand 1999, 100:244-245, discussion 245-246.

349. Choy Y, Fyer AJ, Lipsitz JD: Treatment of specific phobia in adults. Clin Psychol Rev 2007, 27:266-286.

350. Wilhelm FH, Roth WT: Acute and delayed effects of alprazolam on flight phobics during exposure. Behav Res Ther 1997, 35:831-841.

351. Johren P, Jackowski J, Gangler P, Sartory G, Thom A: Fear reduction in patients with dental treatment phobia. Br J Oral Maxillofac Surg 2000, 38:612-616

352. Tschirch FT, Suter K, Froehlich JM, Studler U, Nidecker A, Eckhardt B, Beranek-Chiu J, Surber C, Weishaupt D: Multicenter trial: comparison of two different formulations and application systems of low-dose nasal midazolam for routine magnetic resonance imaging of claustrophobic patients. J Magn Reson Imaging 2008, 28:866-872.

353. Tschirch FT, Gopfert K, Frohlich JM, Brunner G, Weishaupt D: Low-dose intranasal versus oral midazolam for routine body MRI of claustrophobic patients. Eur Radiol 2007, 17:1403-1410.

354. Mather AA, Stein MB, Sareen J: Social anxiety disorder and social fears in the Canadian military: prevalence, comorbidity, impairment, and treatment-seeking. J Psychiatr Res 2010, 44:887-893.

355. Beesdo K, Bittner A, Pine DS, Stein MB, Hofler M, Lieb R, Wittchen HU: Incidence of social anxiety disorder and the consistent risk for secondary depression in the first three decades of life. Arch Gen Psychiatry 2007, 64:903-912.

356. Shields M: Social anxiety disorder-beyond shyness. Health Rep 2004. 15(Suppl):45-61.

357. Magee W, Eaton W, Wittchen H, McGonagle K, Kessler R: Agoraphobia simple phobia, and social phobia in the National Comorbidity Survey. Arch Gen Psychiatry 1996, 53:159-168.

358. Stein M, Walker J, Forde D: Setting diagnostic thresholds for social phobia: considerations from a community survey of social anxiety. Am Psychiatry 1994, 151:408-412
359. Schneier F, Johnson J, Hornig C, Liebowitz M, Weissman M: Social phobia. Comorbidity and morbidity in an epidemiologic sample. Arch Gen Psychiatry 1992, 49:282-288.

360. Ohayon MM, Schatzberg AF: Social phobia and depression: prevalence and comorbidity. J Psychosom Res 2010, 68:235-243.

361. Stein DJ, Ruscio AM, Lee S, Petukhova M, Alonso J, Andrade LH, Benjet C, Bromet E, Demyttenaere K, Florescu S, et al: Subtyping social anxiety disorder in developed and developing countries. Depress Anxiety 2010, 27:390-403.

362. Ramsawh HJ, Raffa SD, Edelen MO, Rende R, Keller MB: Anxiety in middle adulthood: effects of age and time on the 14-year course of panic disorder, social phobia and generalized anxiety disorder. Psychol Med 2009, 39:615-624

363. Ruscio AM, Brown TA, Chiu WT, Sareen J, Stein MB, Kessler RC: Social fears and social phobia in the USA: results from the National Comorbidity Survey Replication. Psychol Med 2008, 38:15-28.

364. Acarturk C, Smit F, de Graaf R, van Straten A, ten Have M, Cuijpers P: Incidence of social phobia and identification of its risk indicators: a model for prevention. Acta Psychiatr Scand 2009, 119:62-70.

365. Kessler R, Stein M, Berglund P: Social phobia subtypes in the National Comorbidity Survey. Am J Psychiatry 1998, 155:613-619.

366. Wong N, Sarver DE, Beidel DC: Quality of life impairments among adults with social phobia: the impact of subtype. J Anxiety Disord 2012, 26:50-57.

367. Aderka IM, Hofmann SG, Nickerson A, Hermesh H, Gilboa-Schechtman E, Marom S: Functional impairment in social anxiety disorder. J Anxiety Disord 2012, 26:393-400.

368. Tolman RM, Himle J, Bybee D, Abelson JL, Hoffman J, Van Etten-Lee M: Impact of social anxiety disorder on employment among women receiving welfare benefits. Psychiatr Serv 2009, 60:61-66.

369. Moitra E, Beard C, Weisberg RB, Keller MB: Occupational impairment and social anxiety disorder in a sample of primary care patients. J Affect Disord 2011, 130:209-212.

370. Acarturk C, Smit F, de Graaf R, van Straten A, Ten Have M, Cuijpers P: Economic costs of social phobia: a population-based study. J Affect Disord 2009, 115:421-429.

371. Smit F, Cuijpers P, Oostenbrink J, Batelaan N, de Graaf R, Beekman A: Costs of nine common mental disorders: implications for curative and preventive psychiatry. J Ment Health Policy Econ 2006, 9:193-200

372. Filho AS, Hetem LA, Ferrari MC, Trzesniak C, Martin-Santos R, Borduqui T, de Lima Osorio F, Loureiro SR, Busatto Filho G, Zuardi AW, Crippa JA: Social anxiety disorder: what are we losing with the current diagnostic criteria? Acta Psychiatr Scand 2010, 121:216-226.

373. Cox BJ, Turnbull DL, Robinson JA, Grant BF, Stein MB: The effect of avoidant personality disorder on the persistence of generalized social anxiety disorder in the general population: results from a longitudinal, nationally representative mental health survey. Depress Anxiety 2011, 28:250-255

374. Kelly MM, Walters C, Phillips KA: Social anxiety and its relationship to functional impairment in body dysmorphic disorder. Behav Ther 2010, 41:143-153.

375. Coles ME, Phillips KA, Menard W, Pagano ME, Fay C, Weisberg RB, Stout RL Body dysmorphic disorder and social phobia: cross-sectional and prospective data. Depress Anxiety 2006, 23:26-33.

376. Book SW, Thomas SE, Randall PK, Randall CL: Paroxetine reduces social anxiety in individuals with a co-occurring alcohol use disorder. $J$ Anxiety Disord 2008, 22:310-318.

377. Bernardi S, Faraone SV, Cortese S, Kerridge BT, Pallanti S, Wang S, Blanco C The lifetime impact of attention deficit hyperactivity disorder: results from the National Epidemiologic Survey on Alcohol and Related Conditions (NESARC). Psychol Med 2012, 42:875-887.

378. Van Ameringen M, Mancini C, Simpson W, Patterson B: Adult attention deficit hyperactivity disorder in an anxiety disorders population. CNS Neurosci Ther 2011, 17:221-226

379. Stern RG, Petti TA, Bopp K, Tobia A: Aripiprazole for the treatment of schizophrenia with co-occurring social anxiety: an open-label crosstaper study. J Clin Psychopharmacol 2009, 29:206-209.

380. Bogels SM, Alden L, Beidel DC, Clark LA, Pine DS, Stein MB, Voncken M: Social anxiety disorder: questions and answers for the DSM-V. Depress Anxiety 2010, 27:168-189. 
381. Taylor S: Meta-analysis of cognitive-behavioral treatments for social phobia. J Behav Ther Exp Psychiatry 1996, 27:1-9.

382. Clark DM, Ehlers A, McManus F, Hackmann A, Fennell M, Campbell H, Flower T, Davenport $C$, Louis $B$ : Cognitive therapy versus fluoxetine in generalized social phobia: a randomized placebo-controlled trial. J Consult Clin Psychol 2003, 71:1058-1067.

383. Clark D, Agras W: The assessment and treatment of performance anxiety in musicians. Am J Psychiatry 1991, 148:598-605

384. Heimberg R, Liebowitz M, Hope D, Schneier F, Holt C, Welkowitz L, Juster H, Campeas R, Bruch M, Cloitre M, et al: Cognitive behavioral group therapy vs phenelzine therapy for social phobia: 12-week outcome. Arch Gen Psychiatry 1998, 55:1133-1141.

385. Otto M, Pollack M, Gould R, Worthington J, McArdle E, Rosenbaum J: A comparison of the efficacy of clonazepam and cognitive-behavioral group therapy for the treatment of social phobia. J Anxiety Disord 2000, 14:345-358

386. Gelernter C, Unde T, Cimbolic P, Arnkoff D, Vittone B, Tancer M, Bartko J: Cognitive-behavioral and pharmacological treatments of social phobia. A controlled study. Arch Gen Psychiatry 1991, 48:938-945.

387. Davidson JR, Foa EB, Huppert JD, Keefe FJ, Franklin ME, Compton JS, Zhao N, Connor KM, Lynch TR, Gadde KM: Fluoxetine, comprehensive cognitive behavioral therapy, and placebo in generalized social phobia. Arch Gen Psychiatry 2004, 61:1005-1013.

388. Liebowitz M, Heimberg R, Schneier F, Hope D, Davies S, Holt C, Goetz D, Juster $\mathrm{H}$, Lin $\mathrm{S}$, Bruch $\mathrm{M}$, et al: Cognitive-behavioral group therapy versus phenelzine in social phobia: long-term outcome. Depress Anxiety 1999, 10:89-98.

389. Haug T, Blomhoff S, Hellstrom K, Holme I, Humble M, Madsbu H, Wold J: Exposure therapy and sertraline in social phobia: I-year follow-up of a randomised controlled trial. Br J Psychiatry 2003, 182:312-318.

390. Stangier U, Heidenreich T, Peitz M, Lauterbach W, Clark DM: Cognitive therapy for social phobia: individual versus group treatment. Behav Res Ther 2003, 41:991-1007.

391. Mortberg E, Clark DM, Sundin O, Aberg Wistedt A: Intensive group cognitive treatment and individual cognitive therapy vs. treatment as usual in social phobia: a randomized controlled trial. Acta Psychiatr Scand 2007, 115:142-154.

392. Hofmann SG: Cognitive mediation of treatment change in social phobia. J Consult Clin Psychol 2004, 72:393-399.

393. Salaberria K, Echeburua E: Long-term outcome of cognitive therapy's contribution to self-exposure in vivo to the treatment of generalized social phobia. Behav Modif 1998, 22:262-284

394. Clark DM, Ehlers A, Hackmann A, McManus F, Fennell M, Grey N, Waddington L, Wild J: Cognitive therapy versus exposure and applied relaxation in social phobia: a randomized controlled trial. J Consult Clin Psychol 2006, 74:568-578.

395. Borgeat F, Stankovic M, Khazaal Y, Rouget BW, Baumann MC, Riquier F, O'Connor K, Jermann F, Zullino D, Bondolfi G: Does the form or the amount of exposure make a difference in the cognitive-behavioral therapy treatment of social phobia? J Nerv Ment Dis 2009, 197:507-513.

396. Smits JA, Powers MB, Buxkamper R, Telch MJ: The efficacy of videotape feedback for enhancing the effects of exposure-based treatment for social anxiety disorder: a controlled investigation. Behav Res Ther 2006, 44:1773-1785.

397. Alden LE, Taylor CT: Relational treatment strategies increase social approach behaviors in patients with generalized social anxiety disorder. J Anxiety Disord 2011, 25:309-318.

398. Lipsitz JD, Gur M, Vermes D, Petkova E, Cheng J, Miller N, Laino J, Liebowitz MR, Fyer AJ: A randomized trial of interpersonal therapy versus supportive therapy for social anxiety disorder. Depress Anxiety 2008, 25:542-553

399. Stangier U, Schramm E, Heidenreich T, Berger M, Clark DM: Cognitive therapy vs interpersonal psychotherapy in social anxiety disorder: a randomized controlled trial. Arch Gen Psychiatry 2011, 68:692-700

400. Borge FM, Hoffart A, Sexton H, Clark DM, Markowitz JC, McManus F: Residential cognitive therapy versus residential interpersonal therapy for social phobia: a randomized clinical trial. J Anxiety Disord 2008, 22:991-1010.

401. Koszycki D, Benger M, Shlik J, Bradwejn J: Randomized trial of a meditation-based stress reduction program and cognitive behavior therapy in generalized social anxiety disorder. Behav Res Ther 2007, 45:2518-2526.

402. Schmidt NB, Richey JA, Buckner JD, Timpano KR: Attention training for generalized social anxiety disorder. J Abnorm Psychol 2009, 118:5-14.

403. Li S, Tan J, Qian M, Liu X: Continual training of attentional bias in social anxiety. Behav Res Ther 2008, 46:905-912.

404. Berger T, Hohl E, Caspar F: Internet-based treatment for social phobia: randomized controlled trial. J Clin Psychol 2009, 65:1021-1035

405. Carlbring P, Gunnarsdottir M, Hedensjo L, Andersson G, Ekselius L, Furmark T: Treatment of social phobia: randomised trial of internetdelivered cognitive-behavioural therapy with telephone support. $\mathrm{Br} J$ Psychiatry 2007, 190:123-128.

406. Titov N, Andrews G, Choi I, Schwencke G, Mahoney A: Shyness 3: randomized controlled trial of guided versus unguided Internet-based CBT for social phobia. Aust N Z J Psychiatry 2008, 42:1030-1040.

407. Titov N, Andrews G, Schwencke G: Shyness 2: treating social phobia online: replication and extension. Aust N Z J Psychiatry 2008, 42:595-605

408. Titov N, Andrews G, Schwencke G, Drobny J, Einstein D: Shyness 1 : distance treatment of social phobia over the Internet. Aust $N Z J$ Psychiatry 2008, 42:585-594.

409. Andersson G, Carlbring P, Holmstrom A, Sparthan E, Furmark T, NilssonIhrfelt E, Buhrman M, Ekselius L: Internet-based self-help with therapist feedback and in vivo group exposure for social phobia: a randomized controlled trial. J Consult Clin Psychol 2006, 74:677-686.

410. Furmark T, Carlbring $P$, Hedman E, Sonnenstein A, Clevberger $P$, Bohman B, Eriksson A, Hallen A, Frykman M, Holmstrom A, et al: Guided and unguided self-help for social anxiety disorder: randomised controlled trial. Br J Psychiatry 2009, 195:440-447.

411. Berger T, Caspar F, Richardson R, Kneubuhler B, Sutter D, Andersson G: Internet-based treatment of social phobia: a randomized controlled trial comparing unguided with two types of guided self-help. Behav Res Ther 2011, 49:158-169.

412. Rapee RM, Abbott MJ, Baillie AJ, Gaston JE: Treatment of social phobia through pure self-help and therapist-augmented self-help. $\mathrm{Br} J$ Psychiatry 2007, 191:246-252

413. Tillfors M, Carlbring P, Furmark T, Lewenhaupt S, Spak M, Eriksson A, Westling $B E$, Andersson $G$ : Treating university students with social phobia and public speaking fears: Internet delivered self-help with or without live group exposure sessions. Depress Anxiety 2008, 25:708-717.

414. Hedman E, Andersson G, Ljotsson B, Andersson E, Ruck C, Mortberg E, Lindefors $\mathrm{N}$ : Internet-based cognitive behavior therapy vs. cognitive behavioral group therapy for social anxiety disorder: a randomized controlled non-inferiority trial. PLoS One 2011, 6:e18001.

415. Andrews $G$, Davies $M$, Titov N: Effectiveness randomized controlled trial of face to face versus Internet cognitive behaviour therapy for social phobia. Aust N Z J Psychiatry 2011, 45:337-340.

416. Hedman E, Andersson E, Ljotsson B, Andersson G, Ruck C, Lindefors N: Cost-effectiveness of Internet-based cognitive behavior therapy vs. cognitive behavioral group therapy for social anxiety disorder: results from a randomized controlled trial. Behav Res Ther 2011, 49:729-736.

417. Prasko J, Dockery C, Horacek J, Houbova P, Kosova J, Klaschka J, Paskova B, Praskova H, Seifertova D, Zalesky R, Hoschl C: Moclobemide and cognitive behavioral therapy in the treatment of social phobia. A six-month controlled study and 24 months follow up. Neuro Endocrinol Lett 2006, 27:473-481

418. Blanco $C$, Heimberg RG, Schneier FR, Fresco DM, Chen $H$, Turk $C L$, Vermes D, Erwin BA, Schmidt AB, Juster HR, et al: A placebo-controlled trial of phenelzine, cognitive behavioral group therapy, and their combination for social anxiety disorder. Arch Gen Psychiatry 2010, 67:286-295

419. Guastella AJ, Richardson R, Lovibond PF, Rapee RM, Gaston JE, Mitchell P, Dadds MR: A randomized controlled trial of $d$-cycloserine enhancement of exposure therapy for social anxiety disorder. Biol Psychiatry 2008, 63:544-549.

420. Hofmann SG, Meuret AE, Smits JA, Simon NM, Pollack MH, Eisenmenger $K$, Shiekh M, Otto MW: Augmentation of exposure therapy with dcycloserine for social anxiety disorder. Arch Gen Psychiatry 2006, 63:298-304

421. Knijnik DZ, Blanco C, Salum GA, Moraes CU, Mombach C, Almeida E, Pereira M, Strapasson A, Manfro GG, Eizirik CL: A pilot study of clonazepam versus psychodynamic group therapy plus clonazepam in 
the treatment of generalized social anxiety disorder. Eur Psychiatry 2008, 23:567-574

422. Watanabe N, Furukawa TA, Chen J, Kinoshita Y, Nakano Y, Ogawa S, Funayama $T$, letsugu $T$, Noda $Y$ : Change in quality of life and their predictors in the long-term follow-up after group cognitive behavioral therapy for social anxiety disorder: a prospective cohort study. BMC Psychiatry 2010, 10:81.

423. Carlbring P, Nordgren LB, Furmark T, Andersson G: Long-term outcome of Internet-delivered cognitive-behavioural therapy for social phobia: a 30-month follow-up. Behav Res Ther 2009, 47:848-850

424. Hedman E, Furmark T, Carlbring P, Ljotsson B, Ruck C, Lindefors N, Andersson G: A 5-year follow-up of internet-based cognitive behavior therapy for social anxiety disorder. J Med Internet Res 2011, 13:e39.

425. Mortberg E, Clark DM, Bejerot S: Intensive group cognitive therapy and individual cognitive therapy for social phobia: sustained improvement at 5-year follow-up. J Anxiety Disord 2011, 25:994-1000.

426. van der Linden $G$, Stein $D$, van Balkom A: The efficacy of the selective serotonin reuptake inhibitors for social anxiety disorder (social phobia): a meta-analysis of randomized controlled trials. Int Clin Psychopharmacol 2000, 15(Suppl 2):S15-23.

427. Canton J, Scott KM, Glue P: Optimal treatment of social phobia: systematic review and meta-analysis. Neuropsychiatr Dis Treat 2012, 8:203-215.

428. Stein DJ, Ipser JC, Balkom AJ: Pharmacotherapy for social phobia. Cochrane Database Syst Rev 2004, :CD001206.

429. Hedges DW, Brown BL, Shwalb DA, Godfrey K, Larcher AM: The efficacy of selective serotonin reuptake inhibitors in adult social anxiety disorder: a meta-analysis of double-blind, placebo-controlled trials. J Psychopharmacol 2007, 21:102-111.

430. Kasper S, Stein D, Loft H, Nil R: Escitalopram in the treatment of social anxiety disorder: Randomised, placebo-controlled, flexible-dosage study. Br J Psychiatry 2005, 186:222-226.

431. Lader M, Stender K, Burger V, Nil R: Efficacy and tolerability of escitalopram in 12- and 24-week treatment of social anxiety disorder: randomised, double-blind, placebo-controlled, fixed-dose study. Depress Anxiety 2004, 19:241-248.

432. Pallanti S, Quercioli L: Resistant social anxiety disorder response to escitalopram. Clin Pract Epidemiol Ment Health 2006, 2:35.

433. van Vliet I, den Boer J, Westenberg H: Psychopharmacological treatment of social phobia; a double blind placebo controlled study with fluvoxamine. Psychopharmacology (Berl) 1994, 115:128-134

434. Stein M, Fyer A, Davidson J, Pollack M, Wiita B: Fluvoxamine treatment of social phobia (social anxiety disorder): a double-blind, placebocontrolled study. Am J Psychiatry 1999, 156:756-760.

435. Asakura S, Tajima O, Koyama T: Fluvoxamine treatment of generalized social anxiety disorder in Japan: a randomized double-blind, placebocontrolled study. Int I Neuropsychopharmacol 2007, 10:263-274.

436. Davidson J, Yaryura-Tobias J, Du Pont R, Stallings L, Barbato L, van dHR, Li D: Fluvoxamine-controlled release formulation for the treatment of generalized social anxiety disorder. I Clin Psychopharmacol 2004, 24:118-125.

437. Westenberg H, Stein D, Yang H, Li D, Barbato L: A double-blind placebocontrolled study of controlled release fluvoxamine for the treatment of generalized social anxiety disorder. J Clin Psychopharmacol 2004, 24:49-55.

438. Allgulander C: Paroxetine in social anxiety disorder: a randomized placebo-controlled study. Acta Psychiatr Scand 1999, 100:193-198.

439. Allgulander C, Mangano R, Zhang J, Dahl AA, Lepola U, Sjodin I, Emilien G: Efficacy of venlafaxine ER in patients with social anxiety disorder: a double-blind, placebo-controlled, parallel-group comparison with paroxetine. Hum Psychopharmacol 2004, 19:387-396.

440. Baldwin D, Bobes J, Stein D, Scharwachter I, Faure M: Paroxetine in social phobia/social anxiety disorder. Randomised, double-blind, placebocontrolled study. Paroxetine Study Group. Br J Psychiatry 1999, 175:120-126.

441. Liebowitz M, Gelenberg A, Munjack D: Venlafaxine extended release vs placebo and paroxetine in social anxiety disorder. Arch Gen Psychiatry 2005, 62:190-198

442. Liebowitz M, Stein M, Tancer M, Carpenter D, Oakes R, Pitts C: A randomized, double-blind, fixed-dose comparison of paroxetine and placebo in the treatment of generalized social anxiety disorder. $J$ Clin Psychiatry 2002, 63:66-74.

443. Stein D, Berk M, Els C, Emsley R, Gittelson L, Wilson D, Oakes R, Hunter B: A double-blind placebo-controlled trial of paroxetine in the management of social phobia (social anxiety disorder) in South Africa. S Afr Med J 1999, 89:402-406.

444. Stein M, Liebowitz M, Lydiard R, Pitts C, Bushnell W, Gergel I: Paroxetine treatment of generalized social phobia (social anxiety disorder): a randomized controlled trial. JAMA 1998, 280:708-713.

445. Katzelnick D, Kobak K, Greist J, Jefferson J, Mantle J, Serlin R: Sertraline for social phobia: a double-blind, placebo-controlled crossover study. Am J Psychiatry 1995, 152:1368-1371.

446. Liebowitz M, De Martinis N, Weihs K, Londborg P, Smith W, Chung H, Fayyad R, Clary C: Efficacy of sertraline in severe generalized social anxiety disorder: results of a double-blind, placebo-controlled study. J Clin Psychiatry 2003, 64:785-792.

447. Van Ameringen M, Lane R, Walker J, Bowen R, Chokka P, Goldner E, Johnston D, Lavallee Y, Nandy S, Pecknold J, et al: Sertraline treatment of generalized social phobia: a 20-week, double-blind, placebo-controlled study. Am J Psychiatry 2001, 158:275-281.

448. Blomhoff S, Haug T, Hellstrom K, Holme I, Humble M, Madsbu H, Wold J: Randomised controlled general practice trial of sertraline, exposure therapy and combined treatment in generalised social phobia. $\mathrm{Br} J$ Psychiatry 2001, 179:23-30.

449. Kobak K, Greist J, Jefferson J, Katzelnick D: Fluoxetine in social phobia: a double-blind, placebo-controlled pilot study. J Clin Psychopharmacol 2002, 22:257-262.

450. Atmaca M, Kuloglu M, Tezcan E, Unal A: Efficacy of citalopram and moclobemide in patients with social phobia: some preliminary findings. Hum Psychopharmacol 2002, 17:401-405.

451. Furmark T, Appel L, Michelgard A, Wahlstedt K, Ahs F, Zancan S, Jacobsson E, Flyckt K, Grohp M, Bergstrom M, et al: Cerebral blood flow changes after treatment of social phobia with the neurokinin-1 antagonist GR205171, citalopram, or placebo. Biol Psychiatry 2005, 58:132-142.

452. Lepola U, Bergtholdt B, St Lambert J, Davy KL, Ruggiero L: Controlledrelease paroxetine in the treatment of patients with social anxiety disorder. J Clin Psychiatry 2004, 65:222-229.

453. Schutters S, van Megen $H$, Van Veen J, Schruers K, Westenberg H: Paroxetine augmentation in patients with generalised social anxiety disorder, non-responsive to mirtazapine or placebo. Hum Psychopharmacol Clin Exp 2011, 26:72-76.

454. Liebowitz M, Mangano R, Bradwejn J, Asnis G: A randomized controlled trial of venlafaxine extended release in generalized social anxiety disorder. J Clin Psychiatry 2005, 66:238-247.

455. Rickels K, Mangano R, Khan A: A double-blind, placebo-controlled study of a flexible dose of venlafaxine ER in adult outpatients with generalized social anxiety disorder. J Clin Psychopharmacol 2004, 24:488-496.

456. Stein M, Pollack M, Bystritsky A, Kelsey J, Mangano R: Efficacy of low and higher dose extended-release venlafaxine in generalized social anxiety disorder: a 6-month randomized controlled trial. Psychopharmacology (Berl) 2005, 177:280-288.

457. Simon NM, Worthington JJ, Moshier SJ, Marks EH, Hoge EA, Brandes M, Delong $\mathrm{H}$, Pollack MH: Duloxetine for the treatment of generalized social anxiety disorder: a preliminary randomized trial of increased dose to optimize response. CNS Spectr 2010, 15:367-373.

458. Gringras M: An uncontrolled trial of clomipramine (Anafranil) in the treatment of phobic and obsessional states in general practice. J Int Med Res 1977, 5(Suppl 5):111-115.

459. Beaumont G: A large open multicentre trial of clomipramine (Anafranil) in the management of phobic disorders. J Int Med Res 1977, 5(Suppl 5):116-123.

460. Simpson H, Schneier F, Campeas R, Marshall R, Fallon B, Davies S, Klein D, Liebowitz M: Imipramine in the treatment of social phobia. J Clin Psychopharmacol 1998, 18:132-135.

461. Liebowitz M, Schneier F, Campeas R, Hollander E, Hatterer J, Fyer A, Gorman J, Papp L, Davies S, Gully R, et al: Phenelzine vs atenolol in social phobia. A placebo-controlled comparison. Arch Gen Psychiatry 1992, 49:290-300. 
462. Versiani M, Nardi A, Mundim F, Alves A, Liebowitz M, Amrein R: Pharmacotherapy of social phobia. A controlled study with moclobemide and phenelzine. Br J Psychiatry 1992, 161:353-360.

463. International Multicenter Clinical Trial Group on Moclobemide in Social Phobia: Moclobemide in social phobia. A double-blind, placebocontrolled clinical study. Eur Arch Psychiatry Clin Neurosci 1997, 247:71-80.

464. Stein D, Cameron A, Amrein R, Montgomery S: Moclobemide is effective and well tolerated in the long-term pharmacotherapy of social anxiety disorder with or without comorbid anxiety disorder. Int Clin Psychopharmacol 2002, 17:161-170.

465. Noyes R, Moroz G, Davidson J, Liebowitz M, Davidson A, Siegel J, Bell J, Cain J, Curlik S, Kent T, et al: Moclobemide in social phobia: a controlled dose-response trial. J Clin Psychopharmacol 1997, 17:247-254.

466. Schneier F, Goetz D, Campeas R, Fallon B, Marshall R, Liebowitz M: Placebo-controlled trial of moclobemide in social phobia. Br J Psychiatry 1998, 172:70-77.

467. Schutters SI, Van Megen HJ, Van Veen JF, Denys DA, Westenberg HG: Mirtazapine in generalized social anxiety disorder: a randomized, double-blind, placebo-controlled study. Int Clin Psychopharmacol 2010, 25:302-304

468. Muehlbacher M, Nickel MK, Nickel C, Kettler C, Lahmann C, Pedrosa Gil F, Leiberich PK, Rother N, Bachler E, Fartacek R, et al: Mirtazapine treatment of social phobia in women: a randomized, double-blind, placebocontrolled study. J Clin Psychopharmacol 2005, 25:580-583.

469. Emmanuel NP, Brawman-Mintzer O, Morton WA, Book SW, Johnson MR, Lorberbaum JP, Ballenger JC, Lydiard RB: Bupropion-SR in treatment of social phobia. Depress Anxiety 2000, 12:111-113.

470. Davidson J, Potts N, Richichi E, Krishnan R, Ford S, Smith R, Wilson W: Treatment of social phobia with clonazepam and placebo. J Clin Psychopharmacol 1993, 13:423-428.

471. Munjack D, Baltazar P, Bohn P, Cabe D, Appleton A: Clonazepam in the treatment of social phobia: a pilot study. J Clin Psychiatry 1990, 51(Suppl):35-40, discussion 50-33.

472. Versiani M, Nardi A, Figuera I, Marques C: Double-blind placebo controlled trial with bromazepam in social phobia. J Bras Psiquiatr 1997, 46:167-171.

473. Seedat S, Stein M: Double-blind, placebo-controlled assessment of combined clonazepam with paroxetine compared with paroxetine monotherapy for generalized social anxiety disorder. J Clin Psychiatry 2004, 65:244-248

474. Pande A, Feltner D, Jefferson J, Davidson J, Pollack M, Stein M, Lydiard R, Futterer R, Robinson P, Slomkowski M, et al: Efficacy of the novel anxiolytic pregabalin in social anxiety disorder: a placebo-controlled multicenter study. J Clin Psychopharmacol 2004, 24:141-149.

475. Feltner DE, Liu-Dumaw M, Schweizer E, Bielski R: Efficacy of pregabalin in generalized social anxiety disorder: results of a double-blind, placebocontrolled, fixed-dose study. Int Clin Psychopharmacol 2011, 26:213-220.

476. Pande A, Davidson J, Jefferson J, Janney C, Katzelnick D, Weisler R, Greist J, Sutherland S: Treatment of social phobia with gabapentin: a placebocontrolled study. J Clin Psychopharmacol 1999, 19:341-348.

477. Urbano MR, Spiegel DR, Laguerta N, Shrader CJ, Rowe DF, Hategan LF: Gabapentin and tiagabine for social anxiety: a randomized, doubleblind, crossover study of 8 adults [Letter]. Prim Care Companion J Clin Psychiatry 2009, 11:123.

478. Zhang W, Connor KM, Davidson JR: Levetiracetam in social phobia: a placebo controlled pilot study. J Psychopharmacol 2005, 19:551-553.

479. Simon NM, Worthington JJ, Doyle AC, Hoge EA, Kinrys G, Fischmann D, Link N, Pollack MH: An open-label study of levetiracetam for the treatment of social anxiety disorder. J Clin Psychiatry 2004, 65:1219-1222.

480. Stein MB, Ravindran LN, Simon NM, Liebowitz MR, Khan A, BrawmanMintzer O, Lydiard RB, Pollack MH: Levetiracetam in generalized social anxiety disorder: a double-blind, randomized controlled trial. J Clin Psychiatry 2010, 71:627-631.

481. Kinrys G, Pollack MH, Simon NM, Worthington JJ, Nardi AE, Versiani M: Valproic acid for the treatment of social anxiety disorder. Int Clin Psychopharmacol 2003, 18:169-172.

482. Dunlop BW, Papp L, Garlow SJ, Weiss PS, Knight BT, Ninan PT: Tiagabine for social anxiety disorder. Hum Psychopharmacol 2007, 22:241-244.

483. Van Ameringen M, Mancini C, Pipe B, Oakman J, Bennett M: An open trial of topiramate in the treatment of generalized social phobia. J Clin Psychiatry 2004, 65:1674-1678.
484. Turner SM, Beidel DC, Jacob RG: Social phobia: a comparison of behavior therapy and atenolol. J Consult Clin Psychol 1994, 62:350-358.

485. van Vliet I, den Boer J, Westenberg H, Pian K: Clinical effects of buspirone in social phobia: a double-blind placebo-controlled study. J Clin Psychiatry 1997, 58:164-168.

486. Ravindran LN, Kim DS, Letamendi AM, Stein MB: A randomized controlled trial of atomoxetine in generalized social anxiety disorder. J Clin Psychopharmacol 2009, 29:561-564.

487. Adler LA, Liebowitz M, Kronenberger W, Oiao M, Rubin R, Hollandbeck M, Deldar A, Schuh K, Durell T: Atomoxetine treatment in adults with attention-deficit/hyperactivity disorder and comorbid social anxiety disorder. Depress Anxiety 2009, 26:212-221.

488. Falloon IR, Lloyd GG, Harpin RE: The treatment of social phobia. Real-life rehearsal with nonprofessional therapists. J Nerv Ment Dis 1981, 169:180-184.

489. Simpson HB, Schneier FR, Marshall RD, Campeas RB, Vermes D, Silvestre J, Davies S, Liebowitz MR: Low dose selegiline (L-Deprenyl) in social phobia. Depress Anxiety 1998, 7:126-129.

490. Villarreal G, Johnson MR, Rubey R, Lydiard RB, Ballanger JC: Treatment of social phobia with the dopamine agonist pergolide. Depress Anxiety 2000, 11:45-47.

491. Van Ameringen M, Mancini C, Wilson C: Buspirone augmentation of selective serotonin reuptake inhibitors (SSRIs) in social phobia. J Affect Disord 1996, 39:115-121

492. Stein M, Sareen J, Hami S, Chao J: Pindolol potentiation of paroxetine for generalized social phobia: a double-blind, placebo-controlled, crossover study. Am J Psychiatry 2001, 158:1725-1727.

493. Barnett S, Kramer M, Casat C, Connor K, Davidson J: Efficacy of olanzapine in social anxiety disorder: a pilot study. J Psychopharmacol 2002, 16:365-368

494. Schutters SI, van Megen HJ, Westenberg HG: Efficacy of quetiapine in generalized social anxiety disorder: results from an open-label study [Letter]. J Clin Psychiatry 2005, 66:540-542.

495. Vaishnavi S, Alamy S, Zhang W, Connor KM, Davidson JR: Quetiapine as monotherapy for social anxiety disorder: a placebo-controlled study. Prog Neuropsychopharmacol Biol Psychiatry 2007, 31:1464-1469.

496. Worthington J, Kinrys G, Wygant L, Pollack M: Aripiprazole as an augmentor of selective serotonin reuptake inhibitors in depression and anxiety disorder patients. Int Clin Psychopharmacol 2005, 20:9-11.

497. Donovan MR, Glue P, Kolluri S, Emir B: Comparative efficacy of antidepressants in preventing relapse in anxiety disorders - a metaanalysis. J Affect Disord 2010, 123:9-16.

498. Greist JH, Liu-Dumaw M, Schweizer E, Feltner D: Efficacy of pregabalin in preventing relapse in patients with generalized social anxiety disorder: results of a double-blind, placebo-controlled 26-week study. Int Clin Psychopharmacol 2011, 26:243-251.

499. Stein D, Westenberg $H$, Yang $H, L i ~ D$, Barbato L: Fluvoxamine CR in the long-term treatment of social anxiety disorder: the 12- to 24-week extension phase of a multicentre, randomized, placebo-controlled trial. Int J Neuropsychopharmacol 2003, 6:317-323.

500. Versiani M, Amrein R, Montgomery S: Social phobia: long-term treatment outcome and prediction of response-a moclobemide study. Int Clin Psychopharmacol 1997, 12:239-254.

501. Fontani V, Mannu P, Castagna A, Rinaldi S: Social anxiety disorder: radio electric asymmetric conveyor brain stimulation versus sertraline. Patient Prefer Adherence 2011, 5:581-586.

502. Kobak K, Taylor L, Warner G, Futterer R: St. John's wort versus placebo in social phobia: results from a placebo-controlled pilot study. J Clin Psychopharmacol 2005, 25:51-58.

503. Gwynn RC, McQuistion HL, McVeigh KH, Garg RK, Frieden TR, Thorpe LE: Prevalence, diagnosis, and treatment of depression and generalized anxiety disorder in a diverse urban community. Psychiatr Serv 2008, 59:641-647.

504. Asnaani A, Richey JA, Dimaite R, Hinton DE, Hofmann SG: A cross-ethnic comparison of lifetime prevalence rates of anxiety disorders. J Nerv Ment Dis 2010, 198:551-555

505. Grant BF, Hasin DS, Stinson FS, Dawson DA, June Ruan W, Goldstein RB, Smith SM, Saha TD, Huang B: Prevalence, correlates, co-morbidity, and comparative disability of DSM-IV generalized anxiety disorder in the USA: results from the National Epidemiologic Survey on Alcohol and Related Conditions. Psychol Med 2005, 35:1747-1759. 
506. Albano A, Chorpita B, Barlow D: Childhood anxiety disorders. In Child psychopathology.. 2 edition. New York, NY: Guilford;Mash E, Barkley R 2003:279-329.

507. Beesdo K, Pine DS, Lieb R, Wittchen HU: Incidence and risk patterns of anxiety and depressive disorders and categorization of generalized anxiety disorder. Arch Gen Psychiatry 2010, 67:47-57.

508. Ansseau M, Fischler B, Dierick M, Albert A, Leyman S, Mignon A: Socioeconomic correlates of generalized anxiety disorder and major depression in primary care: the GADIS II study (Generalized Anxiety and Depression Impact Survey II). Depress Anxiety 2008, 25:506-513.

509. Byers AL, Yaffe K, Covinsky KE, Friedman MB, Bruce ML: High occurrence of mood and anxiety disorders among older adults: the National Comorbidity Survey Replication. Arch Gen Psychiatry 2010, 67:489-496.

510. Mackenzie CS, Reynolds K, Chou KL, Pagura J, Sareen J: Prevalence and correlates of generalized anxiety disorder in a national sample of older adults. Am J Geriatr Psychiatry 2011, 19:305-315.

511. Revicki DA, Travers K, Wyrwich KW, Svedsater H, Locklear J, Mattera MS, Sheehan DV, Montgomery S: Humanistic and economic burden of generalized anxiety disorder in North America and Europe. J Affect Disord 2012, 140:103-112.

512. Baldwin DS, Allgulander C, Bandelow B, Ferre F, Pallanti S: An international survey of reported prescribing practice in the treatment of patients with generalised anxiety disorder. World J Biol Psychiatry 2012, 13:510-516.

513. Weisberg RB, Beard C, Pagano ME, Maki KM, Culpepper L, Keller MB: Impairment and functioning in a sample of primary care patients with generalized anxiety disorder: results from the primary care anxiety project. Prim Care Companion J Clin Psychiatry 2010, 12, doi 10.4088/ PCC.4009m00890blu.

514. Zhu B, Zhao Z, Ye W, Marciniak MD, Swindle R: The cost of comorbid depression and pain for individuals diagnosed with generalized anxiety disorder. J Nerv Ment Dis 2009, 197:136-139.

515. Romera I, Fernandez-Perez S, Montejo AL, Caballero F, Caballero L, Arbesu JA, Delgado-Cohen H, Desaiah D, Polavieja P, Gilaberte I: Generalized anxiety disorder, with or without co-morbid major depressive disorder, in primary care: prevalence of painful somatic symptoms, functioning and health status. J Affect Disord 2010, 127:160-168

516. Garcia-Campayo J, Caballero F, Perez M, Lopez V: Pain related factors in newly diagnosed generalized anxiety disorder patients. Actas Esp Psiquiatr 2012, 40:177-186.

517. Beesdo K, Hoyer J, Jacobi F, Low NC, Hofler M, Wittchen HU: Association between generalized anxiety levels and pain in a community sample: evidence for diagnostic specificity. J Anxiety Disord 2009, 23:684-693.

518. Martens EJ, de Jonge $P, N a$ B, Cohen BE, Lett $H$, Whooley MA: Scared to death? Generalized anxiety disorder and cardiovascular events in patients with stable coronary heart disease:The Heart and Soul Study. Arch Gen Psychiatry 2010, 67:750-758.

519. Tyrer P, Seivewright H, Johnson T: The Nottingham Study of Neurotic Disorder: predictors of 12-year outcome of dysthymic, panic and generalized anxiety disorder. Psychol Med 2004, 34:1385-1394.

520. Covin R, Ouimet AJ, Seeds PM, Dozois DJ: A meta-analysis of CBT for pathological worry among clients with GAD. J Anxiety Disord 2008, 22:108-116.

521. Linden M, Zubraegel D, Baer T, Franke U, Schlattmann P: Efficacy of cognitive behaviour therapy in generalized anxiety disorders. Results of a controlled clinical trial (Berlin CBT-GAD Study). Psychother Psychosom 2005, 74:36-42

522. Borkovec T, Newman M, Pincus A, Lytle R: A component analysis of cognitive-behavioral therapy for generalized anxiety disorder and the role of interpersonal problems. J Consult Clin Psychol 2002, 70:288-298.

523. Ferrero A, Piero A, Fassina S, Massola T, Lanteri A, Daga GA, Fassino S: A 12-month comparison of brief psychodynamic psychotherapy and pharmacotherapy treatment in subjects with generalised anxiety disorders in a community setting. Eur Psychiatry 2007, 22:530-539.

524. Robinson E, Titov N, Andrews G, McIntyre K, Schwencke G, Solley K: Internet treatment for generalized anxiety disorder: a randomized controlled trial comparing clinician vs. technician assistance. PLOS One 2010, 5:e10942.

525. Paxling B, Almlov J, Dahlin M, Carlbring P, Breitholtz E, Eriksson T, Andersson G: Guided internet-delivered cognitive behavior therapy for generalized anxiety disorder: a randomized controlled trial. Cogn Behav Ther 2011, 40:159-173.

526. Gorini A, Pallavicini F, Algeri D, Repetto C, Gaggioli A, Riva G: Virtual reality in the treatment of generalized anxiety disorders. Stud Health Technol Inform 2010, 154:39-43.

527. den Boer PC, Wiersma D, Ten Vaarwerk I, Span MM, Stant AD, Van den Bosch RJ: Cognitive self-therapy for chronic depression and anxiety: a multi-centre randomized controlled study. Psychol Med 2007, 37:329-339.

528. Wells A, Welford M, King P, Papageorgiou C, Wisely J, Mendel E: A pilot randomized trial of metacognitive therapy vs applied relaxation in the treatment of adults with generalized anxiety disorder. Behav Res Ther 2010, 48:429-434

529. Conrad A, Isaac L, Roth WT: The psychophysiology of generalized anxiety disorder: 2. Effects of applied relaxation. Psychophysiology 2008, 45:377-388

530. Dugas MJ, Brillon P, Savard P, Turcotte J, Gaudet A, Ladouceur R, Leblanc R, Gervais NJ: A randomized clinical trial of cognitive-behavioral therapy and applied relaxation for adults with generalized anxiety disorder. Behav Ther 2010, 41:46-58.

531. Dubois O, Salamon R, Germain C, Poirier MF, Vaugeois C, Banwarth B, Mouaffak F, Galinowski A, Olie JP: Balneotherapy versus paroxetine in the treatment of generalized anxiety disorder. Complement Ther Med 2010, 18:1-7.

532. Dugas MJ, Marchand A, Ladouceur R: Further validation of a cognitivebehavioral model of generalized anxiety disorder: diagnostic and symptom specificity. J Anxiety Disord 2005, 19:329-343.

533. Roemer L, Orsillo SM, Salters-Pedneault K: Efficacy of an acceptancebased behavior therapy for generalized anxiety disorder: evaluation in a randomized controlled trial. J Consult Clin Psychol 2008, 76:1083-1089.

534. van der Heiden C, Muris P, van der Molen HT: Randomized controlled trial on the effectiveness of metacognitive therapy and intolerance-ofuncertainty therapy for generalized anxiety disorder. Behav Res Ther 2012, 50:100-109.

535. Hoyer J, Beesdo K, Gloster AT, Runge J, Hofler M, Becker ES: Worry exposure versus applied relaxation in the treatment of generalized anxiety disorder. Psychother Psychosom 2009, 78:106-115.

536. Riskind $\mathrm{JH}$, Williams NL, Joiner TE Jr: The looming cognitive style: A cognitive vulnerability for anxiety disorders. J Social Clin Psychol 2006, 25:779-801.

537. Leichsenring F, Salzer $S$, Jaeger $U$, Kachele $H$, Kreische R, Leweke F, Ruger U, Winkelbach C, Leibing E: Short-term psychodynamic psychotherapy and cognitive-behavioral therapy in generalized anxiety disorder: a randomized, controlled trial. Am J Psychiatry 2009, 166:875-881.

538. Newman MG, Castonguay LG, Borkovec TD, Fisher AJ, Boswell JF, Szkodny LE, Nordberg SS: A randomized controlled trial of cognitivebehavioral therapy for generalized anxiety disorder with integrated techniques from emotion-focused and interpersonal therapies. J Consult Clin Psychol 2011, 79:171-181.

539. Aviram A, Westra HA: The impact of motivational interviewing on resistance in cognitive behavioural therapy for generalized anxiety disorder. Psychother Res 2011, 21:698-708.

540. Westra HA, Arkowitz H, Dozois DJ: Adding a motivational interviewing pretreatment to cognitive behavioral therapy for generalized anxiety disorder: a preliminary randomized controlled trial. J Anxiety Disord 2009, 23:1106-1117

541. Crits-Christoph P, Newman MG, Rickels K, Gallop R, Gibbons MB, Hamilton JL, Ring-Kurtz S, Pastva AM: Combined medication and cognitive therapy for generalized anxiety disorder. J Anxiety Disord 2011, 25:1087-1094.

542. Gosselin P, Ladouceur R, Morin CM, Dugas MJ, Baillargeon L: Benzodiazepine discontinuation among adults with GAD: A randomized trial of cognitivebehavioral therapy. J Consult Clin Psychol 2006, 74:908-919.

543. Salzer S, Winkelbach C, Leweke F, Leibing E, Leichsenring F: Long-term effects of short-term psychodynamic psychotherapy and cognitivebehavioural therapy in generalized anxiety disorder: 12-month followup. Can J Psychiatry 2011, 56:503-508.

544. Davidson J, Bose A, Korotzer A, Zheng H: Escitalopram in the treatment of generalized anxiety disorder: double-blind, placebo controlled, flexible-dose study. Depress Anxiety 2004, 19:234-240.

545. Goodman WK, Bose A, Wang Q: Treatment of generalized anxiety disorder with escitalopram: pooled results from double-blind, placebocontrolled trials. J Affect Disord 2005, 87:161-167. 
546. Bielski RJ, Bose A, Chang CC: A double-blind comparison of escitalopram and paroxetine in the long-term treatment of generalized anxiety disorder. Ann Clin Psychiatry 2005, 17:65-69.

547. Baldwin DS, Huusom AK, Maehlum E: Escitalopram and paroxetine in the treatment of generalised anxiety disorder: randomised, placebocontrolled, double-blind study. Br J Psychiatry 2006, 189:264-272.

548. Bose A, Korotzer A, Gommoll C, Li D: Randomized placebo-controlled trial of escitalopram and venlafaxine XR in the treatment of generalized anxiety disorder. Depress Anxiety 2008, 25:854-861.

549. Bystritsky A, Kerwin L, Feusner JD, Vapnik T: A pilot controlled trial of bupropion XL versus escitalopram in generalized anxiety disorder. Psychopharmacol Bull 2008, 41:46-51.

550. Lenze EJ, Rollman BL, Shear MK, Dew MA, Pollock BG, Ciliberti C, Costantino M, Snyder S, Shi P, Spitznagel E, et al: Escitalopram for older adults with generalized anxiety disorder: a randomized controlled trial. JAMA 2009, 301:295-303.

551. Merideth C, Cutler AJ, She F, Eriksson H: Efficacy and tolerability of extended release quetiapine fumarate monotherapy in the acute treatment of generalized anxiety disorder: a randomized, placebo controlled and active-controlled study. Int Clin Psychopharmacol 2012, 27:40-54.

552. Coric V, Feldman HH, Oren DA, Shekhar A, Pultz J, Dockens RC, Wu X, Gentile KA, Huang SP, Emison E, et al: Multicenter, randomized, doubleblind, active comparator and placebo-controlled trial of a corticotropinreleasing factor receptor-1 antagonist in generalized anxiety disorder. Depress Anxiety 2010, 27:417-425.

553. Kapczinski F, Lima MS, Souza JS, Schmitt R: Antidepressants for generalized anxiety disorder. Cochrane Database Syst Rev 2003, CD003592.

554. Rickels K, Zaninelli R, McCafferty J, Bellew K, lyengar M, Sheehan D: Paroxetine treatment of generalized anxiety disorder: a double-blind, placebo-controlled study. Am J Psychiatry 2003, 160:749-756.

555. Pollack M, Zaninelli R, Goddard A, McCafferty J, Bellew K, Burnham D, lyengar $\mathrm{M}$ : Paroxetine in the treatment of generalized anxiety disorder: results of a placebo-controlled, flexible-dosage trial. I Clin Psychiatry 2001, 62:350-357.

556. Ball S, Kuhn A, Wall D, Shekhar A, Goddard A: Selective serotonin reuptake inhibitor treatment for generalized anxiety disorder: a doubleblind, prospective comparison between paroxetine and sertraline. J Clin Psychiatry 2005, 66:94-99.

557. Bandelow B, Chouinard G, Bobes J, Ahokas A, Eggens I, Liu S, Eriksson H: Extended-release quetiapine fumarate (quetiapine XR): a once-daily monotherapy effective in generalized anxiety disorder. Data from a randomized, double-blind, placebo- and active-controlled study. Int J Neuropsychopharmacol 2010, 13:305-320.

558. Kim TS, Pae CU, Yoon SJ, Bahk WM, Jun TY, Rhee WI, Chae JH: Comparison of venlafaxine extended release versus paroxetine for treatment of patients with generalized anxiety disorder. Psychiatry Clin Neurosci 2006, 60:347-351.

559. Allgulander C, Dahl A, Austin C, Morris P, Sogaard J, Fayyad R, Kutcher S, Clary C: Efficacy of sertraline in a 12-week trial for generalized anxiety disorder. Am J Psychiatry 2004, 161:1642-1649.

560. Brawman-Mintzer O, Knapp RG, Rynn M, Carter RE, Rickels K: Sertraline treatment for generalized anxiety disorder: a randomized, double-blind, placebo-controlled study. J Clin Psychiatry 2006, 67:874-881.

561. Mokhber N, Azarpazhooh MR, Khajehdaluee M, Velayati A, Hopwood M: Randomized, single-blind, trial of sertraline and buspirone for treatment of elderly patients with generalized anxiety disorder. Psychiatry Clin Neurosci 2010, 64:128-133.

562. Varia I, Rauscher F: Treatment of generalized anxiety disorder with citalopram. Int Clin Psychopharmacol 2002, 17:103-107.

563. Simon NM, Zalta AK, Worthington JJ 3rd, Hoge EA, Christian KM, Stevens JC, Pollack MH: Preliminary support for gender differences in response to fluoxetine for generalized anxiety disorder. Depress Anxiety 2006, 23:373-376.

564. Gross PK, Nourse R, Wasser TE, Krulewicz S: Effects of paroxetine CR on depressive and anxiety symptoms: in a community sample of adult Hispanic women with major depression or generalized anxiety disorder. Psychiatry (Edgmont) 2006, 3:64-68.

565. Simon NM, Connor KM, LeBeau RT, Hoge EA, Worthington JJ 3rd, Zhang W, Davidson JR, Pollack MH: Quetiapine augmentation of paroxetine $\mathrm{CR}$ for the treatment of refractory generalized anxiety disorder: preliminary findings. Psychopharmacology (Berl) 2008, 197:675-681.

566. Rynn M, Russell J, Erickson J, Detke MJ, Ball S, Dinkel J, Rickels K, Raskin J: Efficacy and safety of duloxetine in the treatment of generalized anxiety disorder: a flexible-dose, progressive-titration, placebocontrolled trial. Depress Anxiety 2008, 25:182-189.

567. Koponen H, Allgulander C, Erickson J, Dunayevich E, Pritchett Y, Detke MJ, Ball SG, Russell JM: Efficacy of duloxetine for the treatment of generalized anxiety disorder: implications for primary care physicians. Prim Care Companion I Clin Psychiatry 2007, 9:100-107.

568. Wu WY, Wang G, Ball SG, Desaiah D, Ang QQ: Duloxetine versus placebo in the treatment of patients with generalized anxiety disorder in China. Chin Med J (Engl) 2011, 124:3260-3268.

569. Allgulander C, Hartford J, Russell J, Ball S, Erickson J, Raskin J, Rynn M: Pharmacotherapy of generalized anxiety disorder: results of duloxetine treatment from a pooled analysis of three clinical trials. Curr Med Res Opin 2007, 23:1245-1252.

570. Hartford J, Kornstein S, Liebowitz M, Pigott T, Russell J, Detke M, Walker D, Ball S, Dunayevich E, Dinkel J, Erickson J: Duloxetine as an SNRI treatment for generalized anxiety disorder: results from a placebo and activecontrolled trial. Int Clin Psychopharmacol 2007, 22:167-174.

571. Allgulander C, Nutt D, Detke M, Erickson J, Spann M, Walker D, Ball SG, Russell JM: A non-inferiority comparison of duloxetine and venlafaxine in the treatment of adult patients with generalized anxiety disorder. $J$ Psychopharmacol 2008, 22:417-425.

572. Davidson J, Du Pont R, Hedges D, Haskins J: Efficacy, safety, and tolerability of venlafaxine extended release and buspirone in outpatients with generalized anxiety disorder. J Clin Psychiatry 1999, 60:528-535.

573. Nimatoudis I, Zissis N, Kogeorgos J, Theodoropoulou S, Vidalis A, Kaprinis G: Remission rates with venlafaxine extended release in Greek outpatients with generalized anxiety disorder. A double-blind, randomized, placebo controlled study. Int Clin Psychopharmacol 2004, 19:331-336.

574. Rickels K, Pollack M, Sheehan D, Haskins J: Efficacy of extended-release venlafaxine in nondepressed outpatients with generalized anxiety disorder. Am J Psychiatry 2000, 157:968-974.

575. Katz I, Reynolds C, Alexopoulos G, Hackett D: Venlafaxine ER as a treatment for generalized anxiety disorder in older adults: pooled analysis of five randomized placebo-controlled clinical trials. J Am Geriatr Soc 2002, 50:18-25.

576. Montgomery SA, Tobias K, Zornberg GL, Kasper S, Pande AC: Efficacy and safety of pregabalin in the treatment of generalized anxiety disorder: a 6-week, multicenter, randomized, double-blind, placebo-controlled comparison of pregabalin and venlafaxine. J Clin Psychiatry 2006, 67:771-782.

577. Kasper S, Herman B, Nivoli G, Van Ameringen M, Petralia A, Mandel FS, Baldinetti F, Bandelow B: Efficacy of pregabalin and venlafaxine-XR in generalized anxiety disorder: results of a double-blind, placebocontrolled 8-week trial. Int Clin Psychopharmacol 2009, 24:87-96.

578. Allgulander C, Hackett D, Salinas E: Venlafaxine extended release (ER) in the treatment of generalised anxiety disorder: twenty-four-week placebo-controlled dose-ranging study. Br J Psychiatry 2001, 179:15-22.

579. Gelenberg A, Lydiard R, Rudolph R, Aguiar L, Haskins J, Salinas E: Efficacy of venlafaxine extended-release capsules in nondepressed outpatients with generalized anxiety disorder: A 6-month randomized controlled trial. JAMA 2000, 283:3082-3088.

580. Lenox-Smith AJ, Reynolds A: A double-blind, randomised, placebo controlled study of venlafaxine XL in patients with generalised anxiety disorder in primary care. Br J Gen Pract 2003, 53:772-777.

581. Hoehn-Saric R, McLeod D, Zimmerli W: Differential effects of alprazolam and imipramine in generalized anxiety disorder: somatic versus psychic symptoms. J Clin Psychiatry 1988, 49:293-301.

582. Rocca P, Fonzo V, Scotta M, Zanalda E, Ravizza L: Paroxetine efficacy in the treatment of generalized anxiety disorder. Acta Psychiatr Scand 1997, 95:444-450

583. Rickels K, Downing R, Schweizer E, Hassman H: Antidepressants for the treatment of generalized anxiety disorder. A placebo-controlled comparison of imipramine, trazodone, and diazepam. Arch Gen Psychiatry 1993, 50:884-895. 
584. Stein DJ, Ahokas AA, de Bodinat C: Efficacy of agomelatine in generalized anxiety disorder: a randomized, double-blind, placebocontrolled study. J Clin Psychopharmacol 2008, 28:561-566.

585. Stein D, Marquez M, Hoschl C, Ahokas A, Oh K-S, Jarema M, Avedisova A, Vavrusova $\mathrm{L}$, Olivier $\mathrm{V}$ : Efficacy and tolerability of agomelatine in generalized anxiety disorder: A randomised double blind placebo controlled trial with escitalopram as active control [poster]. 28th CINP Congress; Jun 3-7; Stockholm, Sweden 2012.

586. Rothschild AJ, Mahableshwarkar AR, Jacobsen P, Yan M, Sheehan DV: Vortioxetine (Lu AA21004) 5mg in generalized anxiety disorder: Results of an 8-week randomized, double-blind, placebo-controlled clinical trial in the United States. Eur Neuropsychopharmacol 2012, 22:858-866.

587. Bidzan L, Mahableshwarkar AR, Jacobsen P, Yan M, Sheehan DV: Vortioxetine (Lu AA21004) in generalized anxiety disorder: Results of an 8-week, multinational, randomized, double-blind, placebo-controlled clinical trial. Eur Neuropsychopharmacol 2012, 22:847-857.

588. Gambi F, De Berardis D, Campanella D, Carano A, Sepede G, Salini G, Mezzano D, Cicconetti A, Penna L, Salerno RM, Ferro FM: Mirtazapine treatment of generalized anxiety disorder: a fixed dose, open label study. J Psychopharmacol 2005, 19:483-487.

589. Mitte K, Noack P, Steil R, Hautzinger M: A meta-analytic review of the efficacy of drug treatment in generalized anxiety disorder. $J$ Clin Psychopharmacol 2005, 25:141-150.

590. Lydiard R, Ballenger J, Rickels K: A double-blind evaluation of the safety and efficacy of abecarnil, alprazolam, and placebo in outpatients with generalized anxiety disorder. Abecarnil Work Group. I Clin Psychiatry 1997, 58(Suppl 11):11-18.

591. Moller H, Volz H, Reimann I, Stoll K: Opipramol for the treatment of generalized anxiety disorder: a placebo-controlled trial including an alprazolam-treated group. J Clin Psychopharmacol 2001, 21:59-65.

592. Rickels K, Pollack MH, Feltner DE, Lydiard RB, Zimbroff DL, Bielski RJ, Tobias K, Brock JD, Zornberg GL, Pande AC: Pregabalin for treatment of generalized anxiety disorder: a 4-week, multicenter, double-blind, placebo-controlled trial of pregabalin and alprazolam. Arch Gen Psychiatry 2005, 62:1022-1030.

593. Lydiard RB, Rickels K, Herman B, Feltner DE: Comparative efficacy of pregabalin and benzodiazepines in treating the psychic and somatic symptoms of generalized anxiety disorder. Int I Neuropsychopharmacol 2010, 13:229-241.

594. Llorca P, Spadone C, Sol O, Danniau A, Bougerol T, Corruble E, Faruch M, Macher J, Sermet E, Servant D: Efficacy and safety of hydroxyzine in the treatment of generalized anxiety disorder: a 3-month double-blind study. J Clin Psychiatry 2002, 63:1020-1027.

595. Rickels K, Schweizer E, De Martinis N, Mandos L, Mercer C: Gepirone and diazepam in generalized anxiety disorder: a placebo-controlled trial. $J$ Clin Psychopharmacol 1997, 17:272-277.

596. Rickels K, DeMartinis N, Aufdembrinke B: A double-blind, placebocontrolled trial of abecarnil and diazepam in the treatment of patients with generalized anxiety disorder. J Clin Psychopharmacol 2000, 20:12-18.

597. Feltner D, Crockatt J, Dubovsky S, Cohn C, Shrivastava R, Targum S, LiuDumaw M, Carter C, Pande A: A randomized, double-blind, placebocontrolled, fixed-dose, multicenter study of pregabalin in patients with generalized anxiety disorder. J Clin Psychopharmacol 2003, 23:240-249.

598. Laakmann G, Schule C, Lorkowski G, Baghai T, Kuhn K, Ehrentraut S: Buspirone and lorazepam in the treatment of generalized anxiety disorder in outpatients. Psychopharmacology (Berl) 1998, 136:357-366.

599. Fresquet A, Sust M, Lloret A, Murphy M, Carter F, Campbell G, MarionLandais G: Efficacy and safety of lesopitron in outpatients with generalized anxiety disorder. Ann Pharmacother 2000, 34:147-153.

600. Woelk H, Schlafke S: A multi-center, double-blind, randomised study of the lavender oil preparation Silexan in comparison to lorazepam for generalized anxiety disorder. Phytomedicine 2010, 17:94-99.

601. Herrera-Arellano A, Jimenez-Ferrer E, Zamilpa A, Morales-Valdez M, GarciaValencia CE, Tortoriello J: Efficacy and tolerability of a standardized herbal product from galphimia glauca on generalized anxiety disorder. A randomized, double-blind clinical trial controlled with lorazepam. Planta Med 2007, 73:713-717.

602. Khan A, Joyce M, Atkinson S, Eggens I, Baldytcheva I, Eriksson H: A randomized, double-blind study of once-daily extended release quetiapine fumarate (quetiapine XR) monotherapy in patients with generalized anxiety disorder. J Clin Psychopharmacol 2011, 31:418-428.
603. Stein DJ, Bandelow B, Merideth C, Olausson B, Szamosi J, Eriksson H: Efficacy and tolerability of extended release quetiapine fumarate (quetiapine XR) monotherapy in patients with generalised anxiety disorder: an analysis of pooled data from three 8-week placebocontrolled studies. Hum Psychopharmacol 2011, 26:614-628.

604. Katzman MA, Vermani M, Jacobs L, Marcus M, Kong B, Lessard S, Galarraga W, Struzik L, Gendron A: Quetiapine as an adjunctive pharmacotherapy for the treatment of non-remitting generalized anxiety disorder: a flexible-dose, open-label pilot trial. J Anxiety Disord 2008, 22:1480-1486.

605. Altamura AC, Serati M, Buoli M, Dell'Osso B: Augmentative quetiapine in partial/nonresponders with generalized anxiety disorder: a randomized, placebo-controlled study. Int Clin Psychopharmacol 2011, 26:201-205.

606. Brawman-Mintzer O, Knapp RG, Nietert PJ: Adjunctive risperidone in generalized anxiety disorder: a double-blind, placebo-controlled study. J Clin Psychiatry 2005, 66:1321-1325.

607. Pandina GJ, Canuso CM, Turkoz I, Kujawa M, Mahmoud RA: Adjunctive risperidone in the treatment of generalized anxiety disorder: a doubleblind, prospective, placebo-controlled, randomized trial. Psychopharmacol Bull 2007, 40:41-57.

608. Pollack MH, Simon NM, Zalta AK, Worthington JJ, Hoge EA, Mick E, Kinrys G, Oppenheimer J: Olanzapine augmentation of fluoxetine for refractory generalized anxiety disorder: a placebo controlled study. Biol Psychiatry 2006, 59:211-215.

609. Menza MA, Dobkin RD, Marin H: An open-label trial of aripiprazole augmentation for treatment-resistant generalized anxiety disorder [Letter]. J Clin Psychopharmacol 2007, 27:207-210.

610. Gabriel A: The extended-release formulation of quetiapine fumarate (quetiapine XR) adjunctive treatment in partially responsive generalized anxiety disorder (GAD): An open label naturalistic study. Clin Ter 2011, 162:113-118.

611. Lohoff FW, Etemad B, Mandos LA, Gallop R, Rickels K: Ziprasidone treatment of refractory generalized anxiety disorder: a placebocontrolled, double-blind study. J Clin Psychopharmacol 2010, 30:185-189.

612. Snyderman SH, Rynn MA, Rickels K: Open-label pilot study of ziprasidone for refractory generalized anxiety disorder [Letter]. J Clin Psychopharmacol 2005, 25:497-499.

613. Pohl R, Feltner D, Fieve R, Pande A: Efficacy of pregabalin in the treatment of generalized anxiety disorder: double-blind, placebocontrolled comparison of BID versus TID dosing. J Clin Psychopharmacol 2005, 25:151-158

614. Aliyev NA, Aliyev ZN: Valproate (depakine-chrono) in the acute treatment of outpatients with generalized anxiety disorder without psychiatric comorbidity: randomized, double-blind placebo-controlled study. Eur Psychiatry 2008, 23:109-114.

615. Pollack MH, Tiller J, Xie F, Trivedi MH: Tiagabine in adult patients with generalized anxiety disorder: results from 3 randomized, double-blind, placebo-controlled, parallel-group studies. J Clin Psychopharmacol 2008, 28:308-316.

616. Rosenthal M: Tiagabine for the treatment of generalized anxiety disorder: a randomized, open-label, clinical trial with paroxetine as a positive control. J Clin Psychiatry 2003, 64:1245-1249.

617. Rickels K, Shiovitz TM, Ramey TS, Weaver JJ, Knapp LE, Miceli JJ: Adjunctive therapy with pregabalin in generalized anxiety disorder patients with partial response to SSRI or SNRI treatment. Int Clin Psychopharmacol 2012, 27:142-150.

618. Pollack M, Worthington J, Manfro G, Otto M, Zucker B: Abecarnil for the treatment of generalized anxiety disorder: a placebo-controlled comparison of two dosage ranges of abecarnil and buspirone. J Clin Psychiatry 1997, 58(Suppl 11):19-23.

619. Lader M, Scotto J: A multicentre double-blind comparison of hydroxyzine, buspirone and placebo in patients with generalized anxiety disorder. Psychopharmacology (Berl) 1998, 139:402-406.

620. Guaiana G, Barbui C, Cipriani A: Hydroxyzine for generalised anxiety disorder. Cochrane Database Syst Rev 2010, CD006815.

621. Meibach RC, Dunner D, Wilson LG, Ishiki D, Dager SR: Comparative efficacy of propranolol, chlordiazepoxide, and placebo in the treatment of anxiety: a double-blind trial. J Clin Psychiatry 1987, 48:355-358.

622. Feusner JD, Kerwin L, Saxena S, Bystritsky A: Differential efficacy of memantine for obsessive-compulsive disorder vs. generalized anxiety disorder: an open-label trial. Psychopharmacol Bull 2009, 42:81-93. 
623. Baldwin DS, Nutt DJ: On assessing potential efficacy for vortioxetine in generalized anxiety disorder. Eur Neuropsychopharmacol 2012, 22:841-843.

624. Davidson JR, Wittchen HU, Llorca PM, Erickson J, Detke M, Ball SG, Russell JM: Duloxetine treatment for relapse prevention in adults with generalized anxiety disorder: a double-blind placebo-controlled trial. Eur Neuropsychopharmacol 2008, 18:673-681.

625. Allgulander C, Florea I, Huusom AK: Prevention of relapse in generalized anxiety disorder by escitalopram treatment. Int I Neuropsychopharmacol 2006, 9:495-505

626. Stocchi F, Nordera G, Jokinen R, Lepola U, Hewett K, Bryson H, lyengar M Efficacy and tolerability of paroxetine for the long-term treatment of generalized anxiety disorder. J Clin Psychiatry 2003, 64:250-258.

627. Rickels K, Etemad B, Khalid-Khan S, Lohoff FW, Rynn MA, Gallop RJ: Time to relapse after 6 and 12 months' treatment of generalized anxiety disorder with venlafaxine extended release. Arch Gen Psychiatry 2010, 67:1274-1281

628. Feltner D, Wittchen HU, Kavoussi R, Brock J, Baldinetti F, Pande AC: Longterm efficacy of pregabalin in generalized anxiety disorder. Int Clin Psychopharmacol 2008, 23:18-28.

629. Katzman MA, Brawman-Mintzer O, Reyes EB, Olausson B, Liu S, Eriksson H: Extended release quetiapine fumarate (quetiapine XR) monotherapy as maintenance treatment for generalized anxiety disorder: a long-term, randomized, placebo-controlled trial. Int Clin Psychopharmacol 2011, 26:11-24.

630. Bystritsky A, Kaplan JT, Feusner JD, Kerwin LE, Wadekar M, Burock M, Wu AD, lacoboni M: A preliminary study of fMRl-guided rTMS in the treatment of generalized anxiety disorder. J Clin Psychiatry 2008, 69:1092-1098

631. Bystritsky A, Kerwin LE, Feusner JD: A preliminary study of fMRI-guided rTMS in the treatment of generalized anxiety disorder: 6-month followup [Letter]. J Clin Psychiatry 2009, 70:431-432.

632. Kasper S, Gastpar M, Muller WE, Volz HP, Moller HJ, Dienel A, Schlafke S: Silexan, an orally administered Lavandula oil preparation, is effective in the treatment of 'subsyndromal' anxiety disorder: a randomized, double-blind, placebo controlled trial. Int Clin Psychopharmacol 2010, 25:277-287.

633. Miyasaka LS, Atallah AN, Soares BG: Passiflora for anxiety disorder. Cochrane Database Syst Rev 2007, CD004518.

634. Andreatini R, Sartori VA, Seabra ML, Leite JR: Effect of valepotriates (valerian extract) in generalized anxiety disorder: a randomized placebo-controlled pilot study. Phytother Res 2002, 16:650-654.

635. Miyasaka LS, Atallah AN, Soares BG: Valerian for anxiety disorders. Cochrane Database Syst Rev 2006, CD004515.

636. Herring MP, Jacob ML, Suveg C, Dishman RK, O'Connor PJ: Feasibility of exercise training for the short-term treatment of generalized anxiety disorder: a randomized controlled trial. Psychother Psychosom 2012 81:21-28.

637. Pilkington K, Kirkwood G, Rampes H, Cummings M, Richardson J: Acupuncture for anxiety and anxiety disorders-a systematic literature review. Acupunct Med 2007, 25:1-10.

638. Lu CF, Smith LN, Gau CH: Exploring the zen meditation experiences of patients with generalized anxiety disorder: a focus-group approach. J Nurs Res 2012, 20:43-52.

639. Katzman MA, Vermani M, Gerbarg PL, Brown RP, lorio C, Davis M, Cameron C, Tsirgielis D: A multicomponent yoga-based, breath intervention program as an adjunctive treatment in patients suffering from generalized anxiety disorder with or without comorbidities. Int $J$ Yoga 2012, 5:57-65

640. Youngstedt SD, Kline CE, Ginsberg JP, Zielinski MR, Hardin JW: Bright light treatment for high-anxious young adults: a randomized controlled pilot study. Depress Anxiety 2011, 28:324-332.

641. Ruscio AM, Stein DJ, Chiu WT, Kessler RC: The epidemiology of obsessivecompulsive disorder in the National Comorbidity Survey Replication. Mol Psychiatry 2010, 15:53-63.

642. Adam Y, Meinlschmidt G, Gloster AT, Lieb R: Obsessive-compulsive disorder in the community: 12-month prevalence, comorbidity and impairment. Soc Psychiatry Psychiatr Epidemiol 2012, 47:339-349.

643. Rosario-Campos MC, Leckman JF, Mercadante MT, Shavitt RG, Prado HS, Sada P, Zamignani D, Miguel EC: Adults with early-onset obsessivecompulsive disorder. Am J Psychiatry 2001, 158:1899-1903.
644. Veldhuis J, Dieleman JP, Wohlfarth T, Storosum JG, van Den Brink W, Sturkenboom MC, Denys D: Incidence and prevalence of "diagnosed OCD" in a primary care, treatment seeking, population. Int J Psychiatry Clin Pract 2012, 16:85-92.

645. Torres AR, Prince MJ, Bebbington PE, Bhugra D, Brugha TS, Farrell M, Jenkins $R$, Lewis $G$, Meltzer $H$, Singleton N: Obsessive-compulsive disorder: prevalence, comorbidity, impact, and help-seeking in the British National Psychiatric Morbidity Survey of 2000. Am J Psychiatry 2006, 163:1978-1985.

646. Grisham JR, Fullana MA, Mataix-Cols D, Moffitt TE, Caspi A, Poulton R: Risk factors prospectively associated with adult obsessive-compulsive symptom dimensions and obsessive-compulsive disorder. Psychol Med $2011,: 1-12$

647. Hauschildt M, Jelinek L, Randjbar S, Hottenrott B, Moritz S: Generic and illness-specific quality of life in obsessive-compulsive disorder. Behav Cogn Psychother 2010, 38:417-436.

648. Eisen JL, Mancebo MA, Pinto A, Coles ME, Pagano ME, Stout R, Rasmussen SA: Impact of obsessive-compulsive disorder on quality of life. Compr Psychiatry 2006, 47:270-275.

649. Vikas A, Avasthi A, Sharan P: Psychosocial impact of obsessivecompulsive disorder on patients and their caregivers: a comparative study with depressive disorder. Int J Soc Psychiatry 2011, 57:45-56.

650. Rampacher F, Lennertz L, Vogeley A, Schulze-Rauschenbach S, Kathmann N, Falkai $P$, Wagner $M$ : ation processing in patients with $O C D$ compared to patients with unipolar depresEvidence for specific cognitive deficits in visual informsion. Prog Neuropsychopharmacol Biol Psychiatry 2010, 34:984-991.

651. Aigner M, Sachs G, Bruckmuller E, Winklbaur B, Zitterl W, Kryspin-Exner I, Gur R, Katschnig H: Cognitive and emotion recognition deficits in obsessive-compulsive disorder. Psychiatry Res 2007, 149:121-128.

652. Torres AR, Ramos-Cerqueira AT, Ferrao YA, Fontenelle LF, do Rosario MC, Miguel EC: Suicidality in obsessive-compulsive disorder: prevalence and relation to symptom dimensions and comorbid conditions. J Clin Psychiatry 2011, 72:17-26, quiz 119-120.

653. Eaton WW, Martins SS, Nestadt G, Bienvenu OJ, Clarke D, Alexandre P: The burden of mental disorders. Epidemiol Rev 2008, 30:1-14.

654. Leckman JF, Denys D, Simpson HB, Mataix-Cols D, Hollander E, Saxena S, Miguel EC, Rauch SL, Goodman WK, Phillips KA, Stein DJ: Obsessivecompulsive disorder: a review of the diagnostic criteria and possible subtypes and dimensional specifiers for DSM-V. Depress Anxiety 2010, 27:507-527.

655. Abramowitz JS: Effectiveness of psychological and pharmacological treatments for obsessive-compulsive disorder: a quantitative review. J Consult Clin Psychol 1997, 65:44-52.

656. Eddy K, Dutra L, Bradley R, Westen D: A multidimensional meta-analysis of psychotherapy and pharmacotherapy for obsessive-compulsive disorder. Clin Psychol Rev 2004, 24:1011-1030.

657. Noordik E, van der Klink JJ, Klingen EF, Nieuwenhuijsen K, van Dijk FJ: Exposure-in-vivo containing interventions to improve work functioning of workers with anxiety disorder: a systematic review. BMC Public Health 2010, 10:598.

658. Foa E, Liebowitz M, Kozak M, Davies S, Campeas R, Franklin M, Huppert J, Kjernisted K, Rowan V, Schmidt A, et al: Randomized, placebo-controlled trial of exposure and ritual prevention, clomipramine, and their combination in the treatment of obsessive-compulsive disorder. Am J Psychiatry 2005, 162:151-161.

659. Sousa MB, Isolan LR, Oliveira RR, Manfro GG, Cordioli AV: A randomized clinical trial of cognitive-behavioral group therapy and sertraline in the treatment of obsessive-compulsive disorder. J Clin Psychiatry 2006, 67:1133-1139.

660. Belotto-Silva C, Diniz JB, Malavazzi DM, Valerio C, Fossaluza V, Borcato S, Seixas AA, Morelli D, Miguel EC, Shavitt RG: Group cognitive-behavioral therapy versus selective serotonin reuptake inhibitors for obsessivecompulsive disorder: a practical clinical trial. J Anxiety Disord 2012, 26:25-31.

661. Jones MK, Menzies RG: Danger ideation reduction therapy (DIRT) for obsessive-compulsive washers. A controlled trial. Behav Res Ther 1998, 36:959-970

662. Krochmalik A, Jones MK, Menzies RG, Kirkby K: The superiority of danger ideation reduction therapy (DIRT) over exposure and response prevention (ERP) in treating compulsive washing. Behaviour Change 2004, 21:251-268 
663. Jonsson H, Hougaard E, Bennedsen BE: Randomized comparative study of group versus individual cognitive behavioural therapy for obsessive compulsive disorder. Acta Psychiatr Scand 2011, 123:387-397.

664. Jaurrieta N, Jimenez-Murcia S, Alonso P, Granero R, Segalas C, Labad J, Menchon JM: Individual versus group cognitive behavioral treatment for obsessive-compulsive disorder: follow up. Psychiatry Clin Neurosci 2008, 62:697-704.

665. O'Connor K, Freeston MH, Gareau D, Careau Y, Dufour MJ, Aardema F, Todorov C: Group versus individual treatment in obsessions without compulsions. Clin Psychol Psychother 2005, 12:87-96.

666. Belloch A, Cabedo E, Carrio C, Fernandez-Alvarez H, Garcia F, Larsson C: Group versus individual cognitive treatment for obsessive-compulsive disorder: changes in non-OCD symptoms and cognitions at posttreatment and one-year follow-up. Psychiatry Res 2011, 187:174-179.

667. Cabedo E, Belloch A, Carrio C, Larsson C, Fernandez-Alvarez H, Garcia F: Group versus individual cognitive treatment for obsessive-compulsive disorder: changes in severity at post-treatment and one-year follow-up. Behav Cogn Psychother 2010, 38:227-232.

668. Kozak M, Foa E: Mastery of obsessive-compulsive disorder: A cognitivebehavioral approach. San Antonio, TX: The Psychological Corporation; 1997

669. Abramowitz JS, Foa EB, Franklin ME: Exposure and ritual prevention for obsessive-compulsive disorder: effects of intensive versus twice-weekly sessions. J Consult Clin Psychol 2003, 71:394-398.

670. Tolin DF, Diefenbach GJ, Gilliam CM: Stepped care versus standard cognitive-behavioral therapy for obsessive-compulsive disorder: a preliminary study of efficacy and costs. Depress Anxiety 2011, 28:314-323.

671. Twohig MP, Hayes SC, Plumb JC, Pruitt LD, Collins AB, Hazlett-Stevens H, Woidneck MR: A randomized clinical trial of acceptance and commitment therapy versus progressive relaxation training for obsessive-compulsive disorder. J Consult Clin Psychol 2010, 78:705-716.

672. Wilhelm S, Steketee G, Fama JM, Buhlmann U, Teachman BA, Golan E: Modular cognitive therapy for obsessive-compulsive disorder: a wait-list controlled trial. J Cogn Psychother 2009, 23:294-305.

673. Freeston MH, Ladouceur R, Gagnon F, Thibodeau N, Rheaume J, Letarte H, Bujold A: Cognitive-behavioral treatment of obsessive thoughts: a controlled study. J Consult Clin Psychol 1997, 65:405-413.

674. O'Connor KP, Aardema F, Bouthillier D, Fournier S, Guay S, Robillard S, Pelissier MC, Landry P, Todorov C, Tremblay M, Pitre D: Evaluation of an inference-based approach to treating obsessive-compulsive disorder. Cogn Behav Ther 2005, 34:148-163.

675. Park HS, Shin YW, Ha TH, Shin MS, Kim YY, Lee YH, Kwon JS: Effect of cognitive training focusing on organizational strategies in patients with obsessive-compulsive disorder. Psychiatry Clin Neurosci 2006, 60:718-726.

676. Buhlmann U, Deckersbach T, Engelhard I, Cook LM, Rauch SL, Kathmann N, Wilhelm S, Savage CR: Cognitive retraining for organizational impairment in obsessive-compulsive disorder. Psychiatry Res 2006, 144:109-116

677. Hanstede M, Gidron Y, Nyklicek I: The effects of a mindfulness intervention on obsessive-compulsive symptoms in a non-clinical student population. J Nerv Ment Dis 2008, 196:776-779.

678. Simpson HB, Zuckoff AM, Maher MJ, Page JR, Franklin ME, Foa EB, Schmidt $A B$, Wang $Y$ : Challenges using motivational interviewing as an adjunct to exposure therapy for obsessive-compulsive disorder. Behav Res Ther 2010, 48:941-948.

679. Meyer E, Souza F, Heldt E, Knapp P, Cordioli A, Shavitt RG, Leukefeld C: A randomized clinical trial to examine enhancing cognitive-behavioral group therapy for obsessive-compulsive disorder with motivational interviewing and thought mapping. Behav Cogn Psychother 2010, 38:319-336.

680. Nazari H, Momeni N, Jariani M, Tarrahi MJ: Comparison of eye movement desensitization and reprocessing with citalopram in treatment of obsessive-compulsive disorder. Int I Psychiatry Clin Pract 2011, 15:270-274.

681. Tolin DF, Hannan S, Maltby N, Diefenbach GJ, Worhunsky P, Brady RE: A randomized controlled trial of self-directed versus therapist-directed cognitive-behavioral therapy for obsessive-compulsive disorder patients with prior medication trials. Behav Ther 2007, 38:179-191.

682. Lovell K, Cox D, Haddock G, Jones C, Raines D, Garvey R, Roberts C, Hadley S: Telephone administered cognitive behaviour therapy for treatment of obsessive compulsive disorder: randomised controlled non-inferiority trial. BMJ 2006, 333:883.
683. Moritz S, Jelinek L, Hauschildt M, Naber D: How to treat the untreated: effectiveness of a self-help metacognitive training program (myMCT) for obsessive-compulsive disorder. Dialogues Clin Neurosci 2010, 12:209-220

684. Moritz S, Jelinek L: Further evidence for the efficacy of association splitting as a self-help technique for reducing obsessive thoughts. Depress Anxiety 2011, 28:574-581.

685. Andersson E, Enander J, Andren P, Hedman E, Ljotsson B, Hursti T, Bergstrom J, Kaldo V, Lindefors N, Andersson G, Ruck C: Internet-based cognitive behaviour therapy for obsessive-compulsive disorder: a randomized controlled trial. Psychol Med 2012, 1-11.

686. Tumur I, Kaltenthaler E, Ferriter M, Beverley C, Parry G: Computerised cognitive behaviour therapy for obsessive-compulsive disorder: a systematic review. Psychother Psychosom 2007, 76:196-202.

687. Greist JH, Marks IM, Baer L, Kobak KA, Wenzel KW, Hirsch MJ, Mantle JM, Clary CM: Behavior therapy for obsessive-compulsive disorder guided by a computer or by a clinician compared with relaxation as a control. J Clin Psychiatry 2002, 63:138-145.

688. Kenwright M, Marks I, Graham C, Franses A, Mataix-Cols D: Brief scheduled phone support from a clinician to enhance computer-aided self-help for obsessive-compulsive disorder: randomized controlled trial. J Clin Psychol 2005, 61:1499-1508.

689. Lebowitz ER, Panza KE, Su J, Bloch MH: Family accommodation in obsessive-compulsive disorder. Expert Rev Neurother 2012, 12:229-238

690. Rachman S, Elliott CM, Shafran R, Radomsky AS: Separating hoarding from OCD. Behav Res Ther 2009, 47:520-522.

691. Muroff J, Steketee G, Bratiotis C, Ross A: Group cognitive and behavioral therapy and bibliotherapy for hoarding: a pilot trial. Depress Anxiety 2012, 29:597-604

692. Foa EB, Franklin ME, Moser J: Context in the clinic: how well do cognitive-behavioral therapies and medications work in combination? Biol Psychiatry 2002, 52:987-997.

693. Simpson HB, Foa EB, Liebowitz MR, Ledley DR, Huppert JD, Cahill S, Vermes $D$, Schmidt AB, Hembree E, Franklin $M$, et al: A randomized, controlled trial of cognitive-behavioral therapy for augmenting pharmacotherapy in obsessive-compulsive disorder. Am J Psychiatry 2008, 165:621-630.

694. Simpson HB, Liebowitz MR, Foa EB, Kozak MJ, Schmidt AB, Rowan V, Petkova E, Kjernisted K, Huppert JD, Franklin ME, et al: Post-treatment effects of exposure therapy and clomipramine in obsessive-compulsive disorder. Depress Anxiety 2004, 19:225-233.

695. van Oppen P, van Balkom AJ, de Haan E, van Dyck R: Cognitive therapy and exposure in vivo alone and in combination with fluvoxamine in obsessive-compulsive disorder: a 5-year follow-up. J Clin Psychiatry 2005, 66:1415-1422

696. O'Connor KP, Aardema F, Robillard S, Guay S, Pelissier MC, Todorov C, Borgeat F, Leblanc V, Grenier S, Doucet P: Cognitive behaviour therapy and medication in the treatment of obsessive-compulsive disorder. Acta Psychiatr Scand 2006, 113:408-419.

697. Kordon A, Kahl KG, Broocks A, Voderholzer U, Rasche-Rauchle H, Hohagen F: Clinical outcome in patients with obsessive-compulsive disorder after discontinuation of SRI treatment: results from a two-year follow-up. Eur Arch Psychiatry Clin Neurosci 2005, 255:48-50.

698. Chasson GS, Buhlmann U, Tolin DF, Rao SR, Reese HE, Rowley T, Welsh KS, Wilhelm S: Need for speed: evaluating slopes of OCD recovery in behavior therapy enhanced with d-cycloserine. Behav Res Ther 2010 48:675-679.

699. Kushner MG, Kim SW, Donahue C, Thuras P, Adson D, Kotlyar M, McCabe J, Peterson J, Foa EB: D-cycloserine augmented exposure therapy for obsessive-compulsive disorder. Biol Psychiatry 2007, 62:835-838

700. Wilhelm S, Buhlmann U, Tolin DF, Meunier SA, Pearlson GD, Reese HE, Cannistraro P, Jenike MA, Rauch SL: Augmentation of behavior therapy with d-cycloserine for obsessive-compulsive disorder. Am J Psychiatry 2008, 165:335-341, quiz 409.

701. Storch EA, Merlo $L$, Bengtson M, Murphy TK, Lewis MH, Yang MC, Jacob ML, Larson M, Hirsh A, Fernandez M, et al: D-cycloserine does not enhance exposure-response prevention therapy in obsessivecompulsive disorder. Int Clin Psychopharmacol 2007, 22:230-237.

702. Braga DT, Manfro GG, Niederauer K, Cordioli AV: Full remission and relapse of obsessive-compulsive symptoms after cognitive-behavioral group therapy: a two-year follow-up. Rev Bras Psiquiatr 2010, 32:164-168. 
703. Whittal ML, Robichaud M, Thordarson DS, McLean PD: Group and individual treatment of obsessive-compulsive disorder using cognitive therapy and exposure plus response prevention: a 2-year follow-up of two randomized trials. J Consult Clin Psychol 2008, 76:1003-1014.

704. Anand N, Sudhir PM, Math SB, Thennarasu K, Janardhan Reddy YC: Cognitive behavior therapy in medication non-responders with obsessive-compulsive disorder: a prospective 1-year follow-up study. J Anxiety Disord 2011, 25:939-945.

705. Stein DJ, Andersen EW, Tonnoir B, Fineberg N: Escitalopram in obsessivecompulsive disorder: a randomized, placebo-controlled, paroxetinereferenced, fixed-dose, 24-week study. Curr Med Res Opin 2007, 23:701-711.

706. Khan MN, Hotiana UA, Ahmad S: Escitalopram in the treatment of obsessive-compulsive disorder: a double blind placebo control trial. J Ayub Med Coll Abbottabad 2007, 19:58-63.

707. Shim G, Park HY, Jang JH, Kim E, Hwang JY, Kim SN, Jang GE, Kwon JS: What is the optimal dose of escitalopram for the treatment of obsessive-compulsive disorder? A naturalistic open-label study. Int Clin Psychopharmacol 2011, 26:284-290.

708. Dougherty DD, Jameson M, Deckersbach T, Loh R, Thompson-Hollands J, Jenike $M$, Keuthen NJ: Open-label study of high $(30 \mathrm{mg}$ ) and moderate (20 mg) dose escitalopram for the treatment of obsessive-compulsive disorder. Int Clin Psychopharmacol 2009, 24:306-311.

709. Rabinowitz I, Baruch Y, Barak Y: High-dose escitalopram for the treatment of obsessive-compulsive disorder. Int Clin Psychopharmacol 2008, 23:49-53.

710. Bergeron R, Ravindran A, Chaput $Y$, Goldner E, Swinson R, van AM, Austin C, Hadrava V: Sertraline and fluoxetine treatment of obsessivecompulsive disorder: results of a double-blind, 6-month treatment study. J Clin Psychopharmacol 2002, 22:148-154.

711. Piccinelli M, Pini S, Bellantuono C, Wilkinson G: Efficacy of drug treatment in obsessive-compulsive disorder. A meta-analytic review. Br J Psychiatry 1995, 166:424-443.

712. Zitterl W, Meszaros K, Hornik K, Twaroch T, Dossenbach M, Zitterl-Eglseer K, Zapotoczky HG: Efficacy of fluoxetine in Austrian patients with obsessive-compulsive disorder. Wien Klin Wochenschr 1999, 111:439-442.

713. Greist JH, Jefferson JW, Kobak KA, Katzelnick DJ, Serlin RC: Efficacy and tolerability of serotonin transport inhibitors in obsessive-compulsive disorder. A meta-analysis. Arch Gen Psychiatry 1995, 52:53-60.

714. Ackerman DL, Greenland S: Multivariate meta-analysis of controlled drug studies for obsessive-compulsive disorder. J Clin Psychopharmacol 2002, 22:309-317.

715. Sayyah M, Boostani H, Pakseresht S, Malayeri A: Comparison of silybum marianum (L.) gaertn. with fluoxetine in the treatment of obsessivecompulsive disorder. Prog Neuropsychopharmacol Biol Psychiatry 2010, 34:362-365.

716. Lopez-Ibor JJ Jr., Saiz J, Cottraux J, Note I, Vinas R, Bourgeois M, Hernandez M, Gomez-Perez JC: Double-blind comparison of fluoxetine versus clomipramine in the treatment of obsessive compulsive disorder. Eur Neuropsychopharmacol 1996, 6:111-118.

717. Mundo E, Maina G, Uslenghi C: Multicentre, double-blind, comparison of fluvoxamine and clomipramine in the treatment of obsessivecompulsive disorder. Int Clin Psychopharmacol 2000, 15:69-76.

718. Mundo E, Rouillon F, Figuera M, Stigler M: Fluvoxamine in obsessivecompulsive disorder: similar efficacy but superior tolerability in comparison with clomipramine. Hum Psychopharmacol 2001, 16:461-468.

719. Hollander E, Koran LM, Goodman WK, Greist JH, Ninan PT, Yang H, Li D, Barbato LM: A double-blind, placebo-controlled study of the efficacy and safety of controlled-release fluvoxamine in patients with obsessivecompulsive disorder. J Clin Psychiatry 2003, 64:640-647.

720. Zohar J, Judge R: Paroxetine versus clomipramine in the treatment of obsessive-compulsive disorder. OCD Paroxetine Study Investigators. $\mathrm{Br} J$ Psychiatry 1996, 169:468-474.

721. Denys $D$, van der Wee $N$, van Megen HJ, Westenberg HG: A double blind comparison of venlafaxine and paroxetine in obsessive-compulsive disorder. J Clin Psychopharmacol 2003, 23:568-575.

722. Hollander E, Allen A, Steiner M, Wheadon DE, Oakes R, Burnham DB: Acute and long-term treatment and prevention of relapse of obsessivecompulsive disorder with paroxetine. J Clin Psychiatry 2003, 64:1113-1121.

723. Hoehn-Saric R, Ninan P, Black D, Stahl S, Greist J, Lydiard B, McElroy S, Zajecka J, Chapman D, Clary C, Harrison W: Multicenter double-blind comparison of sertraline and desipramine for concurrent obsessivecompulsive and major depressive disorders. Arch Gen Psychiatry 2000, 57:76-82.

724. Bisserbe J, Lane R, Flament M: A double-blind comparison of sertraline and clomipramine in outpatients with obsessive compulsive disorder. Eur Psychiatry 1997, 12:82-93.

725. Ninan PT, Koran LM, Kiev A, Davidson JR, Rasmussen SA, Zajecka JM, Robinson DG, Crits-Christoph P, Mandel FS, Austin C: High-dose sertraline strategy for nonresponders to acute treatment for obsessivecompulsive disorder: a multicenter double-blind trial. J Clin Psychiatry 2006, 67:15-22.

726. Montgomery S, Kasper S, Stein D, Bang Hedegaard K, Lemming O: Citalopram $20 \mathrm{mg}, 40 \mathrm{mg}$ and $60 \mathrm{mg}$ are all effective and well tolerated compared with placebo in obsessive-compulsive disorder. Int Clin Psychopharmacol 2001, 16:75-86.

727. Pallanti S, Quercioli L, Bruscoli M: Response acceleration with mirtazapine augmentation of citalopram in obsessive-compulsive disorder patients without comorbid depression: a pilot study. J Clin Psychiatry 2004, 65:1394-1399.

728. Vulink NC, Denys D, Fluitman SB, Meinardi JC, Westenberg HG: Quetiapine augments the effect of citalopram in non-refractory obsessivecompulsive disorder: a randomized, double-blind, placebo-controlled study of 76 patients. J Clin Psychiatry 2009, 70:1001-1008.

729. Pallanti S, Quercioli L, Koran LM: Citalopram intravenous infusion in resistant obsessive-compulsive disorder: an open trial. J Clin Psychiatry 2002, 63:796-801.

730. Marazziti D, Golia F, Consoli G, Presta S, Pfanner C, Carlini M, Mungai F, Catena Dell'osso M: Effectiveness of long-term augmentation with citalopram to clomipramine in treatment-resistant OCD patients. CNS Spectr 2008, 13:971-976

731. Albert U, Aguglia E, Maina G, Bogetto F: Venlafaxine versus clomipramine in the treatment of obsessive-compulsive disorder: a preliminary singleblind, 12-week, controlled study. J Clin Psychiatry 2002, 63:1004-1009.

732. Yaryura-Tobias JA, Neziroglu FA: Venlafaxine in obsessive-compulsive disorder [Letter]. Arch Gen Psychiatry 1996, 53:653-654.

733. Denys D, van Megen HJ, van der Wee N, Westenberg HG: A double-blind switch study of paroxetine and venlafaxine in obsessive-compulsive disorder. J Clin Psychiatry 2004, 65:37-43.

734. Dell'osso B, Mundo E, Marazziti D, Altamura AC: Switching from serotonin reuptake inhibitors to duloxetine in patients with resistant obsessive compulsive disorder: a case series. J Psychopharmacol 2008, 22:210-213.

735. Blay S, Black DW: A case of obsessive-compulsive disorder responding to duloxetine. Prim Care Companion J Clin Psychiatry 2007, 9:234-235.

736. Yeh YW, Chen CH, Kuo SC, Wang SC, Chen CK, Feng HM: High-dose duloxetine for treatment-resistant obsessive-compulsive disorder: a case report with sustained full remission. Clin Neuropharmacol 2009, 32:174-176.

737. Vallejo J, Olivares J, Marcos T, Bulbena A, Menchon JM: Clomipramine versus phenelzine in obsessive-compulsive disorder. A controlled clinical trial. Br J Psychiatry 1992, 161:665-670.

738. Jenike MA, Baer L, Minichiello WE, Rauch SL, Buttolph ML: Placebocontrolled trial of fluoxetine and phenelzine for obsessive-compulsive disorder. Am J Psychiatry 1997, 154:1261-1264.

739. Joffe R, Swinson R: Tranylcypromine in primary obsessive-compulsive disorder. J Anxiety Disord 1990, 4:365-367.

740. Stein DJ, Spadaccini E, Hollander E: Meta-analysis of pharmacotherapy trials for obsessive-compulsive disorder. Int Clin Psychopharmacol 1995, 10:11-18.

741. Kobak KA, Greist JH, Jefferson JW, Katzelnick DJ, Henk HJ: Behavioral versus pharmacological treatments of obsessive compulsive disorder: a meta-analysis. Psychopharmacology (Berl) 1998, 136:205-216.

742. Fallon BA, Liebowitz MR, Campeas R, Schneier FR, Marshall R, Davies S, Goetz D, Klein DF: Intravenous clomipramine for obsessive-compulsive disorder refractory to oral clomipramine: a placebo-controlled study. Arch Gen Psychiatry 1998, 55:918-924.

743. Koran LM, Sallee FR, Pallanti S: Rapid benefit of intravenous pulse loading of clomipramine in obsessive-compulsive disorder. Am J Psychiatry 1997, 154:396-401.

744. Koran LM, Aboujaoude E, Ward H, Shapira NA, Sallee FR, Gamel N, Elliott M: Pulse-loaded intravenous clomipramine in treatment-resistant obsessive-compulsive disorder. J Clin Psychopharmacol 2006, 26:79-83. 
745. Goodman WK, Price LH, Delgado PL, Palumbo J, Krystal JH, Nagy LM Rasmussen SA, Heninger GR, Charney DS: Specificity of serotonin reuptake inhibitors in the treatment of obsessive-compulsive disorder. Comparison of fluvoxamine and desipramine. Arch Gen Psychiatry 1990, 47:577-585.

746. Diniz JB, Shavitt RG, Pereira CA, Hounie AG, Pimentel I, Koran LM, Dainesi SM, Miguel EC: Quetiapine versus clomipramine in the augmentation of selective serotonin reuptake inhibitors for the treatment of obsessive-compulsive disorder: a randomized, open-label trial. J Psychopharmacol 2010, 24:297-307

747. Diniz JB, Shavitt RG, Fossaluza V, Koran L, Pereira CA, Miguel EC: A doubleblind, randomized, controlled trial of fluoxetine plus quetiapine or clomipramine versus fluoxetine plus placebo for obsessive-compulsive disorder. J Clin Psychopharmacol 2011, 31:763-768.

748. Koran L, Gamel N, Choung H, Smith E, Aboujaoude E: Mirtazapine for obsessive-compulsive disorder: an open trial followed by double-blind discontinuation. J Clin Psychiatry 2005, 66:515-520.

749. Vulink NC, Denys D, Westenberg HG: Bupropion for patients with obsessive-compulsive disorder: an open-label, fixed-dose study. J Clin Psychiatry 2005, 66:228-230.

750. Muscatello MR, Bruno A, Pandolfo G, Mico U, Scimeca G, Romeo VM, Santoro V, Settineri S, Spina E, Zoccali RA: Effect of aripiprazole augmentation of serotonin reuptake inhibitors or clomipramine in treatment-resistant obsessive-compulsive disorder: a double-blind placebo-controlled study. J Clin Psychopharmacol 2011, 31:174-179.

751. Pessina E, Albert U, Bogetto F, Maina G: Aripiprazole augmentation of serotonin reuptake inhibitors in treatment-resistant obsessivecompulsive disorder: a 12-week open-label preliminary study. Int Clin Psychopharmacol 2009, 24:265-269.

752. Ak M, Bulut SD, Bozkurt A, Ozsahin A: Aripiprazole augmentation of serotonin reuptake inhibitors in treatment-resistant obsessivecompulsive disorder: a 10-week open-label study. Adv Ther 2011 28:341-348.

753. Delle Chiaie R, Scarciglia P, Pasquini M, Caredda M, Biondi M: Aripiprazole augmentation in patients with resistant obsessive compulsive disorder: a pilot study. Clin Pract Epidemiol Ment Health 2011, 7:107-111.

754. Sayyah M, Boostani H, Ghaffari SM, Hoseini A: Effects of aripiprazole augmentation in treatment-resistant obsessive-compulsive disorder (a double blind clinical trial). Depress Anxiety 2012, 29:850-854

755. Selvi Y, Atli A, Aydin A, Besiroglu L, Ozdemir P, Ozdemir O: The comparison of aripiprazole and risperidone augmentation in selective serotonin reuptake inhibitor-refractory obsessive-compulsive disorder: a single-blind, randomised study. Hum Psychopharmacol 2011, 26:51-57.

756. McDougle C, Epperson C, Pelton G, Wasylink S, Price L: A double-blind, placebo-controlled study of risperidone addition in serotonin reuptake inhibitor-refractory obsessive-compulsive disorder. Arch Gen Psychiatry 2000, 57:794-801.

757. Hollander E, Baldini Rossi N, Sood E, Pallanti S: Risperidone augmentation in treatment-resistant obsessive-compulsive disorder: a double-blind, placebo-controlled study. Int I Neuropsychopharmacol 2003, 6:397-401.

758. Li X, May RS, Tolbert LC, Jackson WT, Flournoy JM, Baxter LR: Risperidone and haloperidol augmentation of serotonin reuptake inhibitors in refractory obsessive-compulsive disorder: a crossover study. J Clin Psychiatry 2005, 66:736-743.

759. Erzegovesi S, Guglielmo E, Siliprandi F, Bellodi L: Low-dose risperidone augmentation of fluvoxamine treatment in obsessive-compulsive disorder: a double-blind, placebo-controlled study. Eur Neuropsychopharmacol 2005, 15:69-74.

760. Maina G, Pessina E, Albert U, Bogetto F: 8-week, single-blind, randomized trial comparing risperidone versus olanzapine augmentation of serotonin reuptake inhibitors in treatment-resistant obsessivecompulsive disorder. Eur Neuropsychopharmacol 2008, 18:364-372.

761. Simpson HB, Foa EB, Liebowitz MR, Huppert JD, Cahill S, Maher MJ McLean CP, Bender J Jr., Marcus SM, Williams MT, et al: Cognitivebehavioral therapy vs risperidone for augmenting serotonin reuptake inhibitors in obsessive-compulsive disorder: a randomized clinical trial. JAMA Psychiatry 2013, 70:1190-1199.

762. Bystritsky A, Ackerman DL, Rosen RM, Vapnik T, Gorbis E, Maidment KM, Saxena S: Augmentation of serotonin reuptake inhibitors in refractory obsessive-compulsive disorder using adjunctive olanzapine: a placebocontrolled trial. J Clin Psychiatry 2004, 65:565-568.
763. Shapira NA, Ward HE, Mandoki M, Murphy TK, Yang MC, Blier P, Goodman WK: A double-blind, placebo-controlled trial of olanzapine addition in fluoxetine-refractory obsessive-compulsive disorder. Biol Psychiatry 2004, 55:553-555.

764. Fineberg NA, Sivakumaran T, Roberts A, Gale T: Adding quetiapine to SR in treatment-resistant obsessive-compulsive disorder: a randomized controlled treatment study. Int Clin Psychopharmacol 2005, 20:223-226.

765. Carey PD, Vythilingum B, Seedat S, Muller JE, van Ameringen M, Stein DJ: Quetiapine augmentation of SRIs in treatment refractory obsessivecompulsive disorder: a double-blind, randomised, placebo-controlled study. BMC Psychiatry 2005, 5:5.

766. Denys D, de Geus F, van Megen H, Westenberg H: A double blind, randomized, placebo-controlled trial of quetiapine addition in patients with obsessive-compulsive disorder refractory to serotonin reuptake inhibitors. J Clin Psychiatry 2004, 65:1040-1048.

767. Savas HA, Yumru M, Ozen ME: Quetiapine and ziprasidone as adjuncts in treatment-resistant obsessive-compulsive disorder: a retrospective comparative study. Clin Drug Investig 2008, 28:439-442.

768. Kordon A, Wahl K, Koch N, Zurowski B, Anlauf M, Vielhaber K, Kahl KG, Broocks A, Voderholzer U, Hohagen F: Quetiapine addition to serotonin reuptake inhibitors in patients with severe obsessive-compulsive disorder: a double-blind, randomized, placebo-controlled study. J Clin Psychopharmacol 2008, 28:550-554.

769. McDougle CJ, Goodman WK, Leckman JF, Lee NC, Heninger GR, Price LH: Haloperidol addition in fluvoxamine-refractory obsessive-compulsive disorder. A double-blind, placebo-controlled study in patients with and without tics. Arch Gen Psychiatry 1994, 51:302-308.

770. Metin O, Yazici K, Tot S, Yazici AE: Amisulpiride augmentation in treatment resistant obsessive-compulsive disorder: an open trial. Hum Psychopharmacol 2003, 18:463-467.

771. Hollander E, Kaplan A, Stahl SM: A double-blind, placebo-controlled trial of clonazepam in obsessive-compulsive disorder. World J Biol Psychiatry 2003, 4:30-34

772. Crockett BA, Churchill E, Davidson JR: A double-blind combination study of clonazepam with sertraline in obsessive-compulsive disorder. Ann Clin Psychiatry 2004, 16:127-132.

773. Hewlett WA, Vinogradov S, Agras WS: Clomipramine, clonazepam, and clonidine treatment of obsessive-compulsive disorder. J Clin Psychopharmacol 1992, 12:420-430.

774. Dannon P, Sasson Y, Hirschmann S, lancu I, Grunhaus L, Zohar J: Pindolo augmentation in treatment-resistant obsessive compulsive disorder: a double-blind placebo controlled trial. Eur Neuropsychopharmacol 2000, 10:165-169.

775. Mundo E, Guglielmo E, Bellodi L: Effect of adjuvant pindolol on the antiobsessional response to fluvoxamine: a double-blind, placebocontrolled study. Int Clin Psychopharmacol 1998, 13:219-224.

776. Blier $P$, Bergeron R: Sequential administration of augmentation strategies in treatment-resistant obsessive-compulsive disorder: preliminary findings. Int Clin Psychopharmacol 1996, 11:37-44.

777. Sayyah M, Boostani H, Pakseresht S, Malayeri A: A preliminary randomized double-blind clinical trial on the efficacy of celecoxib as an adjunct in the treatment of obsessive-compulsive disorder. Psychiatry Res 2011, 189:403-406.

778. Askari N, Moin M, Sanati M, Tajdini M, Hosseini SM, Modabbernia A, Najand B, Salimi S, Tabrizi M, Ashrafi M, et al: Granisetron adjunct to fluvoxamine for moderate to severe obsessive-compulsive disorder: a randomized, double-blind, placebo-controlled trial. CNS Drugs 2012, 26:883-892.

779. Rodriguez Cl, Kegeles LS, Levinson A, Feng T, Marcus SM, Vermes D, Flood P, Simpson HB: Randomized controlled crossover trial of ketamine in obsessive-compulsive disorder: proof-of-concept. Neuropsychopharmacology 2013, 38:2475-2483.

780. Bloch MH, Wasylink S, Landeros-Weisenberger A, Panza KE, Billingslea E, Leckman JF, Krystal JH, Bhagwagar Z, Sanacora G, Pittenger C: Effects of ketamine in treatment-refractory obsessive-compulsive disorder. Biol Psychiatry 2012, 72:964-970.

781. Aboujaoude E, Barry JJ, Gamel N: Memantine augmentation in treatment-resistant obsessive-compulsive disorder: an open-label trial. $J$ Clin Psychopharmacol 2009, 29:51-55.

782. Stewart SE, Jenike EA, Hezel DM, Stack DE, Dodman NH, Shuster L, Jenike MA: A single-blinded case-control study of memantine in severe obsessive-compulsive disorder. J Clin Psychopharmacol 2010, 30:34-39. 
783. Ghaleiha A, Entezari N, Modabbernia A, Najand B, Askari N, Tabrizi M, Ashrafi M, Hajiaghaee R, Akhondzadeh S: Memantine add-on in moderate to severe obsessive-compulsive disorder: randomized double-blind placebo-controlled study. J Psychiatr Res 2013, 47:175-180.

784. Pallanti S, Bernardi S, Antonini S, Singh N, Hollander E: Ondansetron augmentation in treatment-resistant obsessive-compulsive disorder: a preliminary, single-blind, prospective study. CNS Drugs 2009, 23:1047-1055.

785. Soltani F, Sayyah M, Feizy F, Malayeri A, Siahpoosh A, Motlagh I: A doubleblind, placebo-controlled pilot study of ondansetron for patients with obsessive-compulsive disorder. Hum Psychopharmacol 2010, 25:509-513.

786. Afshar H, Roohafza H, Mohammad-Beigi H, Haghighi M, Jahangard L, Shokouh P, Sadeghi M, Hafezian H: N-acetylcysteine add-on treatment in refractory obsessive-compulsive disorder: a randomized, double-blind, placebo-controlled trial. J Clin Psychopharmacol 2012, 32:797-803.

787. Van Ameringen M, Patterson B, Simpson W, Turna J: N-acetylcysteine augmentation in treatment resistant obsessive compulsive disorder: A case series. J Obsess Compul Rel Dis 2013, 2:48-52.

788. Coric V, Taskiran S, Pittenger C, Wasylink S, Mathalon DH, Valentine G Saksa J, Wu YT, Gueorguieva R, Sanacora G, et al: Riluzole augmentation in treatment-resistant obsessive-compulsive disorder: an open-label trial. Biol Psychiatry 2005, 58:424-428.

789. Pittenger C, Kelmendi B, Wasylink S, Bloch MH, Coric V: Riluzole augmentation in treatment-refractory obsessive-compulsive disorder: a series of 13 cases, with long-term follow-up. J Clin Psychopharmacol 2008, 28:363-367

790. McDougle CJ, Price LH, Goodman WK, Charney DS, Heninger GR: A controlled trial of lithium augmentation in fluvoxamine-refractory obsessive-compulsive disorder: lack of efficacy. J Clin Psychopharmacol 1991, 11:175-184

791. Pigott TA, Pato MT, L'Heureux F, Hill JL, Grover GN, Bernstein SE, Murphy DL: A controlled comparison of adjuvant lithium carbonate or thyroid hormone in clomipramine-treated patients with obsessivecompulsive disorder. J Clin Psychopharmacol 1991, 11:242-248.

792. Jenike MA, Baer $L$, Buttolph L: Buspirone augmentation of fluoxetine in patients with obsessive compulsive disorder. J Clin Psychiatry 1991, 52:13-14

793. Pigott TA, L'Heureux F, Hill JL, Bihari K, Bernstein SE, Murphy DL: A doubleblind study of adjuvant buspirone hydrochloride in clomipraminetreated patients with obsessive-compulsive disorder. J Clin Psychopharmacol 1992, 12:11-18.

794. Rodriguez Cl, Bender J Jr., Marcus SM, Snape M, Rynn M, Simpson HB: Minocycline augmentation of pharmacotherapy in obsessivecompulsive disorder: an open-label trial [Letter]. J Clin Psychiatry 2010, 71:1247-1249.

795. Rubio G, Jimenez-Arriero MA, Martinez-Gras I, Manzanares J, Palomo T: The effects of topiramate adjunctive treatment added to antidepressants in patients with resistant obsessive-compulsive disorder. J Clin Psychopharmacol 2006, 26:341-344.

796. Berlin HA, Koran LM, Jenike MA, Shapira NA, Chaplin W, Pallanti S, Hollander E: Double-blind, placebo-controlled trial of topiramate augmentation in treatment-resistant obsessive-compulsive disorder. $J$ Clin Psychiatry 2011, 72:716-721

797. Mowla A, Khajeian AM, Sahraian A, Chohedri AH, Kashkoli F: Topiramate augmentation in resistant OCD: a double-blind placebo-controlled clinical trial. CNS Spectr 2010, 15:613-617.

798. Van Ameringen M, Mancini C, Patterson B, Bennett M: Topiramate augmentation in treatment-resistant obsessive-compulsive disorder: a retrospective, open-label case series. Depress Anxiety 2006, 23:1-5.

799. Bruno A, Mico U, Pandolfo G, Mallamace D, Abenavoli E, Di Nardo F, D'Arrigo C, Spina E, Zoccali RA, Muscatello MR: Lamotrigine augmentation of serotonin reuptake inhibitors in treatment-resistant obsessivecompulsive disorder: a double-blind, placebo-controlled study. $J$ Psychopharmacol 2012, 26:1456-1462.

800. Kumar TC, Khanna S: Lamotrigine augmentation of serotonin re-uptake inhibitors in obsessive-compulsive disorder. Aust N Z J Psychiatry 2000, 34:527-528.

801. Di Nicola M, Tedeschi D, Martinotti G, De Vita O, Monetta M, Pozzi G, Janiri L: Pregabalin augmentation in treatment-resistant obsessivecompulsive disorder: a 16-week case series. J Clin Psychopharmacol 2011, 31:675-677.
802. Oulis P, Mourikis I, Konstantakopoulos G: Pregabalin augmentation in treatment-resistant obsessive-compulsive disorder. Int Clin Psychopharmacol 2011, 26:221-224.

803. Onder E, Tural U, Gokbakan M: Does gabapentin lead to early symptom improvement in obsessive-compulsive disorder? Eur Arch Psychiatry Clin Neurosci 2008, 258:319-323.

804. Cora-Locatelli G, Greenberg BD, Martin J, Murphy DL: Gabapentin augmentation for fluoxetine-treated patients with obsessive-compulsive disorder [Letter]. J Clin Psychiatry 1998, 59:480-481.

805. Goldsmith TB, Shapira NA, Keck PE Jr.: Rapid remission of OCD with tramadol hydrochloride [Letter]. Am J Psychiatry 1999, 156:660-661.

806. Shapira NA, Keck PE Jr., Goldsmith TD, McConville BJ, Eis M, McElroy SL: Open-label pilot study of tramadol hydrochloride in treatmentrefractory obsessive-compulsive disorder. Depress Anxiety 1997, 6:170-173.

807. Amiaz R, Fostick L, Gershon A, Zohar J: Naltrexone augmentation in OCD: a double-blind placebo-controlled cross-over study. Eur Neuropsychopharmacol 2008, 18:455-461.

808. Koran LM, Aboujaoude E, Bullock KD, Franz B, Gamel N, Elliott M: Doubleblind treatment with oral morphine in treatment-resistant obsessivecompulsive disorder. J Clin Psychiatry 2005, 66:353-359.

809. Soomro GM, Altman D, Rajagopal S, Oakley-Browne M: Selective serotonin re-uptake inhibitors (SSRIs) versus placebo for obsessive compulsive disorder (OCD). Cochrane Database Syst Rev 2008, CD001765.

810. Stein DJ, Andersen EW, Overo KF: Response of symptom dimensions in obsessive-compulsive disorder to treatment with citalopram or placebo. Rev Bras Psiquiatr 2007, 29:303-307.

811. Stein DJ, Carey PD, Lochner C, Seedat S, Fineberg N, Andersen EW: Escitalopram in obsessive-compulsive disorder: response of symptom dimensions to pharmacotherapy. CNS Spectr 2008, 13:492-498.

812. Landeros-Weisenberger A, Bloch MH, Kelmendi B, Wegner R, Nudel J, Dombrowski P, Pittenger C, Krystal JH, Goodman WK, Leckman JF, Coric V: Dimensional predictors of response to SRI pharmacotherapy in obsessive-compulsive disorder. J Affect Disord 2010, 121:175-179.

813. Koran LM, Hanna GL, Hollander E, Nestadt G, Simpson HB: Practice guideline for the treatment of patients with obsessive-compulsive disorder. Am J Psychiatry 2007, 164:5-53.

814. Decloedt EH, Stein DJ: Current trends in drug treatment of obsessivecompulsive disorder. Neuropsychiatr Dis Treat 2010, 6:233-242.

815. Ipser JC, Carey P, Dhansay Y, Fakier N, Seedat S, Stein DJ: Pharmacotherapy augmentation strategies in treatment-resistant anxiety disorders. Cochrane Database Syst Rev 2006, CD005473.

816. Bloch MH, Landeros-Weisenberger A, Kelmendi B, Coric V, Bracken MB, Leckman JF: A systematic review: antipsychotic augmentation with treatment refractory obsessive-compulsive disorder. Mol Psychiatry 2006, 11:622-632.

817. Komossa K, Depping AM, Meyer M, Kissling W, Leucht S: Secondgeneration antipsychotics for obsessive compulsive disorder. Cochrane Database Syst Rev 2010, CD008141

818. Fineberg NA, Tonnoir B, Lemming O, Stein DJ: Escitalopram prevents relapse of obsessive-compulsive disorder. Eur Neuropsychopharmacol 2007, 17:430-439.

819. Koran LM, Hackett E, Rubin A, Wolkow R, Robinson D: Efficacy of sertraline in the long-term treatment of obsessive-compulsive disorder. Am J Psychiatry 2002, 159:88-95.

820. Romano S, Goodman W, Tamura R, Gonzales J: Long-term treatment of obsessive-compulsive disorder after an acute response: a comparison of fluoxetine versus placebo. J Clin Psychopharmacol 2001, 21:46-52.

821. Katz RJ, DeVeaugh-Geiss J, Landau P: Clomipramine in obsessivecompulsive disorder. Biol Psychiatry 1990, 28:401-414.

822. Rasmussen S, Hackett E, DuBoff E, Greist J, Halaris A, Koran LM, Liebowitz M, Lydiard RB, McElroy S, Mendels J, O'Connor K: A 2-year study of sertraline in the treatment of obsessive-compulsive disorder. Int Clin Psychopharmacol 1997, 12:309-316.

823. Ravizza L, Barzega G, Bellino S, Bogetto F, Maina G: Drug treatment of obsessive-compulsive disorder (OCD): long-term trial with clomipramine and selective serotonin reuptake inhibitors (SSRIs). Psychopharmacol Bull 1996, 32:167-173.

824. Koran LM, Bromberg D, Hornfeldt CS, Shepski JC, Wang S, Hollander E: Extended-release fluvoxamine and improvements in quality of life in patients with obsessive-compulsive disorder. Compr Psychiatry 2010, 51:373-379 
825. Mantovani A, Lisanby SH, Pieraccini F, Ulivelli M, Castrogiovanni P, Rossi S: Repetitive transcranial magnetic stimulation (rTMS) in the treatment of obsessive-compulsive disorder (OCD) and Tourette's syndrome (TS). Int J Neuropsychopharmacol 2006, 9:95-100.

826. Kumar N, Chadda RK: Augmentation effect of repetitive transcranial magnetic stimulation over the supplementary motor cortex in treatment refractory patients with obsessive compulsive disorder. Indian J Psychiatry 2011, 53:340-342.

827. Mantovani A, Simpson HB, Fallon BA, Rossi S, Lisanby SH: Randomized sham-controlled trial of repetitive transcranial magnetic stimulation in treatment-resistant obsessive-compulsive disorder. Int J Neuropsychopharmacol 2010, 13:217-227

828. Ruffini C, Locatelli M, Lucca A, Benedetti F, Insacco C, Smeraldi E: Augmentation effect of repetitive transcranial magnetic stimulation over the orbitofrontal cortex in drug-resistant obsessive-compulsive disorder patients: a controlled investigation. Prim Care Companion J Clin Psychiatry 2009, 11:226-230.

829. Sachdev PS, Loo CK, Mitchell PB, McFarquhar TF, Malhi GS: Repetitive transcranial magnetic stimulation for the treatment of obsessive compulsive disorder: a double-blind controlled investigation. Psychol Med 2007, 37:1645-1649

830. Sarkhel S, Sinha VK, Praharaj SK: Adjunctive high-frequency right prefrontal repetitive transcranial magnetic stimulation (rTMS) was not effective in obsessive-compulsive disorder but improved secondary depression. J Anxiety Disord 2010, 24:535-539.

831. Prasko J, Paskova B, Zalesky R, Novak T, Kopecek M, Bares M, Horacek J: The effect of repetitive transcranial magnetic stimulation (rTMS) on symptoms in obsessive compulsive disorder. A randomized, double blind, sham controlled study. Neuro Endocrinol Lett 2006, 27:327-332.

832. Greenberg BD, Gabriels LA, Malone DA Jr., Rezai AR, Friehs GM, Okun MS, Shapira NA, Foote KD, Cosyns PR, Kubu CS, et al: Deep brain stimulation of the ventral internal capsule/ventral striatum for obsessivecompulsive disorder: worldwide experience. Mol Psychiatry 2010, 15:64-79.

833. Goodman WK, Foote KD, Greenberg BD, Ricciuti N, Bauer R, Ward H, Shapira NA, Wu SS, Hill CL, Rasmussen SA, Okun MS: Deep brain stimulation for intractable obsessive compulsive disorder: pilot study using a blinded, staggered-onset design. Biol Psychiatry 2010, 67:535-542.

834. Abelson JL, Curtis GC, Sagher O, Albucher RC, Harrigan M, Taylor SF, Martis B, Giordani B: Deep brain stimulation for refractory obsessivecompulsive disorder. Biol Psychiatry 2005, 57:510-516.

835. Liu K, Zhang H, Liu C, Guan Y, Lang L, Cheng Y, Sun B, Wang H, Zuo C, Pan L, et al: Stereotactic treatment of refractory obsessive compulsive disorder by bilateral capsulotomy with 3 years follow-up. J Clin Neurosci 2008, 15:622-629.

836. Ruck C, Karlsson A, Steele JD, Edman G, Meyerson BA, Ericson K, Nyman H, Asberg M, Svanborg P: Capsulotomy for obsessive-compulsive disorder: long-term follow-up of 25 patients. Arch Gen Psychiatry 2008, 65:914-921.

837. Lopes AC, Greenberg BD, Noren G, Canteras MM, Busatto GF, de Mathis ME, Taub A, D'Alcante CC, Hoexter MQ, Gouvea FS, et al: Treatment of resistant obsessive-compulsive disorder with ventral capsular/ventral striatal gamma capsulotomy: a pilot prospective study. J Neuropsychiatry Clin Neurosci 2009, 21:381-392.

838. Csigo K, Harsanyi A, Demeter G, Rajkai C, Nemeth A, Racsmany M: Longterm follow-up of patients with obsessive-compulsive disorder treated by anterior capsulotomy: a neuropsychological study. J Affect Disord 2010, 126:198-205.

839. Ruck C, Larsson KJ, Mataix-Cols D: Predictors of medium and long-term outcome following capsulotomy for obsessive-compulsive disorder: one site may not fit all. Eur Neuropsychopharmacol 2012, 22:406-414.

840. Jung HH, Kim CH, Chang JH, Park YG, Chung SS, Chang JW: Bilateral anterior cingulotomy for refractory obsessive-compulsive disorder: Long-term follow-up results. Stereotact Funct Neurosurg 2006, 84:184-189.

841. Dougherty DD, Baer L, Cosgrove GR, Cassem EH, Price BH, Nierenberg AA, Jenike MA, Rauch SL: Prospective long-term follow-up of 44 patients who received cingulotomy for treatment-refractory obsessivecompulsive disorder. Am J Psychiatry 2002, 159:269-275.

842. Kim CH, Chang JW, Koo MS, Kim JW, Suh HS, Park IH, Lee HS: Anterior cingulotomy for refractory obsessive-compulsive disorder. Acta Psychiatr Scand 2003, 107:283-290.
843. Krisanaprakornkit T, Krisanaprakornkit W, Piyavhatkul N, Laopaiboon M: Meditation therapy for anxiety disorders. Cochrane Database Syst Rev 2006, CD004998.

844. Abrantes AM, Strong DR, Cohn A, Cameron AY, Greenberg BD, Mancebo MC, Brown RA: Acute changes in obsessions and compulsions following moderate-intensity aerobic exercise among patients with obsessive-compulsive disorder. J Anxiety Disord 2009, 23:923-927.

845. Brown RA, Abrantes AM, Strong DR, Mancebo MC, Menard J, Rasmussen SA, Greenberg BD: A pilot study of moderate-intensity aerobic exercise for obsessive compulsive disorder. J Nerv Ment Dis 2007, 195:514-520.

846. Pakseresht S, Boostani H, Sayyah M: Extract of valerian root (Valeriana officinalis L.) vs. placebo in treatment of obsessive-compulsive disorder: a randomized double-blind study. J Complement Integr Med 2011, 8, 10.2202/1553-3840.1465.

847. Taylor LH, Kobak KA: An open-label trial of St. John's Wort (Hypericum perforatum) in obsessive-compulsive disorder. J Clin Psychiatry 2000, 61:575-578

848. Van Ameringen M, Mancini C, Patterson B, Boyle MH: Post-traumatic stress disorder in Canada. CNS Neurosci Ther 2008, 14:171-181.

849. Pietrzak RH, Goldstein RB, Southwick SM, Grant BF: Prevalence and Axis I comorbidity of full and partial posttraumatic stress disorder in the United States: results from Wave 2 of the National Epidemiologic Survey on Alcohol and Related Conditions. J Anxiety Disord 2011, 25:456-465.

850. Darves-Bornoz JM, Alonso J, de Girolamo G, de Graaf R, Haro JM, KovessMasfety V, Lepine JP, Nachbaur G, Negre-Pages L, Vilagut G, Gasquet I: Main traumatic events in Europe: PTSD in the European study of the epidemiology of mental disorders survey. J Trauma Stress 2008, 21:455-462.

851. Stein $M B$, Walker JR, Hazen $A L$, Forde DR: Full and partial posttraumatic stress disorder: findings from a community survey. Am J Psychiatry 1997, 154:1114-1119.

852. Bravo-Mehmedbasic A, Kucukalic A, Kulenovic AD, Suljic E: Impact of chronic posttraumatic stress disorder on the quality of life of war survivors. Psychiatr Danub 2010, 22:430-435.

853. Pietrzak RH, Goldstein MB, Malley JC, Johnson DC, Southwick SM: Subsyndromal posttraumatic stress disorder is associated with health and psychosocial difficulties in veterans of Operations Enduring Freedom and Iraqi Freedom. Depress Anxiety 2009, 26:739-744.

854. Westphal M, Olfson M, Gameroff MJ, Wickramaratne P, Pilowsky DJ, Neugebauer R, Lantigua R, Shea S, Neria Y: Functional impairment in adults with past posttraumatic stress disorder: findings from primary care. Depress Anxiety 2011, 28:686-695.

855. Rona RJ, Jones M, Iversen A, Hull L, Greenberg N, Fear NT, Hotopf M, Wessely S: The impact of posttraumatic stress disorder on impairment in the UK military at the time of the Iraq war. J Psychiatr Res 2009, 43:649-655

856. Asmundson GJ, Wright KD, Stein MB: Pain and PTSD symptoms in female veterans. Eur J Pain 2004, 8:345-350.

857. Poundja J, Fikretoglu D, Guay S, Brunet A: Validation of the French version of the brief pain inventory in Canadian veterans suffering from traumatic stress. J Pain Symptom Manage 2007, 33:720-726.

858. Villano CL, Rosenblum A, Magura S, Fong C, Cleland C, Betzler TF: Prevalence and correlates of posttraumatic stress disorder and chronic severe pain in psychiatric outpatients. J Rehabil Res Dev 2007, 44:167-178.

859. Lowe B, Kroenke K, Spitzer RL, Williams JB, Mussell M, Rose M, Wingenfeld K, Sauer N, Spitzer C: Trauma exposure and posttraumatic stress disorder in primary care patients: cross-sectional criterion standard study. J Clin Psychiatry 2011, 72:304-312.

860. Babson K, Feldner M, Badour C, Trainor C, Blumenthal H, Sachs-Ericsson N, Schmidt N: Posttraumatic stress and sleep: differential relations across types of symptoms and sleep problems. J Anxiety Disord 2011, 25:706-713.

861. Hirsch KA: Sexual dysfunction in male Operation Enduring Freedom/ Operation Iraqi Freedom patients with severe post-traumatic stress disorder. Mil Med 2009, 174:520-522.

862. Lagarde G, Doyon J, Brunet A: Memory and executive dysfunctions associated with acute posttraumatic stress disorder. Psychiatry Res 2010, 177:144-149. 
863. Reinhard MJ, Wolf G, Cozolino L: Using the MMPI to assess reported cognitive disturbances and somatization as a core feature of complex PTSD. J Trauma Dissociation 2010, 11:57-72.

864. Frewen PA, Dozois DJ, Neufeld RW, Lanius RA: Meta-analysis of alexithymia in posttraumatic stress disorder. J Trauma Stress 2008, 21:243-246.

865. Sareen J, Houlahan T, Cox BJ, Asmundson GJ: Anxiety disorders associated with suicidal ideation and suicide attempts in the National Comorbidity Survey. J Nerv Ment Dis 2005, 193:450-454.

866. Kartha A, Brower V, Saitz R, Samet JH, Keane TM, Liebschutz J: The impact of trauma exposure and post-traumatic stress disorder on healthcare utilization among primary care patients. Med Care 2008, 46:388-393.

867. Fikretoglu D, Brunet A, Guay S, Pedlar D: Mental health treatment seeking by military members with posttraumatic stress disorder: findings on rates, characteristics, and predictors from a nationally representative Canadian military sample. Can J Psychiatry 2007, 52:103-110

868. Sareen J, Belik SL, Stein MB, Asmundson GJ: Correlates of perceived need for mental health care among active military personnel. Psychiatr Serv 2010, 61:50-57.

869. Nacasch N, Fostick L, Zohar J: High prevalence of obsessive-compulsive disorder among posttraumatic stress disorder patients. Eur Neuropsychopharmacol 2011, 21:876-879.

870. Cougle JR, Feldner MT, Keough ME, Hawkins KA, Fitch KE: Comorbid panic attacks among individuals with posttraumatic stress disorder: associations with traumatic event exposure history, symptoms, and impairment. J Anxiety Disord 2010, 24:183-188.

871. Ginzburg K, Ein-Dor T, Solomon Z: Comorbidity of posttraumatic stress disorder, anxiety and depression: a 20-year longitudinal study of war veterans. J Affect Disord 2010, 123:249-257.

872. Post $L M$, Zoellner LA, Youngstrom E, Feeny NC: Understanding the relationship between co-occurring PTSD and MDD: symptom severity and affect. J Anxiety Disord 2011, 25:1123-1130.

873. Fetzner MG, McMillan KA, Sareen J, Asmundson GJ: What is the association between traumatic life events and alcohol abuse/ dependence in people with and without PTSD? Findings from a nationally representative sample. Depress Anxiety 2011, 28:632-638.

874. Pagura J, Stein MB, Bolton JM, Cox BJ, Grant B, Sareen J: Comorbidity of borderline personality disorder and posttraumatic stress disorder in the U.S. population. J Psychiatr Res 2010, 44:1190-1198.

875. Friedman MJ, Resick PA, Bryant RA, Brewin CR: Considering PTSD for DSM-5. Depress Anxiety 2011, 28:750-769.

876. Rose S, Bisson J, Churchill R, Wessely S: Psychological debriefing for preventing post traumatic stress disorder (PTSD). Cochrane Database Syst Rev 2002, CD000560.

877. van Emmerik AA, Kamphuis JH, Hulsbosch AM, Emmelkamp PM: Single session debriefing after psychological trauma: a meta-analysis. Lancet 2002, 360:766-771.

878. Roberts NP, Kitchiner NJ, Kenardy J, Bisson J: Multiple session early psychological interventions for the prevention of post-traumatic stress disorder. Cochrane Database Syst Rev 2009, CD006869.

879. Kornor H, Winje D, Ekeberg O, Weisaeth L, Kirkehei I, Johansen K, Steiro A: Early trauma-focused cognitive-behavioural therapy to prevent chronic post-traumatic stress disorder and related symptoms: a systematic review and meta-analysis. BMC Psychiatry 2008, 8:81.

880. Roberts NP, Kitchiner NJ, Kenardy J, Bisson JI: Early psychological interventions to treat acute traumatic stress symptoms. Cochrane Database Syst Rev 2010, CD007944.

881. Gelpin E, Bonne O, Peri T, Brandes D, Shalev A: Treatment of recent trauma survivors with benzodiazepines: a prospective study. J Clin Psychiatry 1996, 57:390-394.

882. Mellman TA, Bustamante V, David D, Fins Al: Hypnotic medication in the aftermath of trauma [letter]. J Clin Psychiatry 2002, 63:1183-1184.

883. Fowler M, Garza TH, Slater TM, Maani CV, McGhee LL: The relationship between gabapentin and pregabalin and posttraumatic stress disorder in burned servicemembers. J Burn Care Res 2012, 33:612-618.

884. Vaiva G, Ducroca F, Jezequel $K$, Averland B, Lestavel P, Brunet A, Marmar C Immediate treatment with propranolol decreases posttraumatic stress disorder two months after trauma. Biol Psychiatry 2003, 54:947-949.

885. Tarsitani L, De Santis V, Mistretta M, Parmigiani G, Zampetti G, Roselli V, Vitale D, Tritapepe L, Biondi M, Picardi A: Treatment with beta-blockers and incidence of post-traumatic stress disorder after cardiac surgery: a prospective observational study. J Cardiothorac Vasc Anesth 2011, 26:265-269.

886. Sharp S, Thomas C, Rosenberg L, Rosenberg M, Meyer W 3rd: Propranolol does not reduce risk for acute stress disorder in pediatric burn trauma. J Trauma 2010, 68:193-197.

887. Pastrana Jimenez Jl, Catalina Romero C, Garcia Dieguez N, Lopez-Ibor Alino JJ: Pharmacological treatment of acute stress disorder with propranolol and hypnotics. Actas Esp Psiquiatr 2007, 35:351-358.

888. McGhee LL, Maani CV, Garza TH, Desocio PA, Gaylord KM, Black IH: The effect of propranolol on posttraumatic stress disorder in burned service members. J Burn Care Res 2009, 30:92-97.

889. Pitman R, Sanders K, Zusman R, Healy A, Cheema F, Lasko N, Cahill L, Orr S: Pilot study of secondary prevention of posttraumatic stress disorder with propranolol. Biol Psychiatry 2002, 51:189-192.

890. Stoddard FJ Jr., Luthra R, Sorrentino EA, Saxe GN, Drake J, Chang Y, Levine JB, Chedekel DS, Sheridan RL: A randomized controlled trial of sertraline to prevent posttraumatic stress disorder in burned children. J Child Adolesc Psychopharmacol 2011, 21:469-477.

891. Bryant RA, Creamer M, O'Donnell M, Silove D, McFarlane AC: A study of the protective function of acute morphine administration on subsequent posttraumatic stress disorder. Biol Psychiatry 2009, 65:438-440.

892. Stoddard FJ Jr., Sorrentino EA, Ceranoglu TA, Saxe G, Murphy JM, Drake JE, Ronfeldt $\mathrm{H}$, White GW, Kagan J, Snidman N, et al: Preliminary evidence for the effects of morphine on posttraumatic stress disorder symptoms in one- to four-year-olds with burns. J Burn Care Res 2009, 30:836-843.

893. Saxe G, Geary M, Bedard K, Bosquet M, Miller A, Koenen K, Stoddard F, Moulton $\mathrm{S}$ : Separation anxiety as a mediator between acute morphine administration and PTSD symptoms in injured children. Ann N Y Acad Sci 2006, 1071:41-45.

894. Holbrook TL, Galarneau MR, Dye JL, Quinn K, Dougherty AL: Morphine use after combat injury in Iraq and post-traumatic stress disorder. $N$ Engl J Med 2010, 362:110-117.

895. Stewart CL, Wrobel TA: Evaluation of the efficacy of pharmacotherapy and psychotherapy in treatment of combat-related post-traumatic stress disorder: a meta-analytic review of outcome studies. Mil Med 2009, 174:460-469.

896. Nijdam MJ, Gersons BP, Reitsma JB, de Jongh A, Olff M: Brief eclectic psychotherapy $\mathrm{v}$. eye movement desensitisation and reprocessing therapy for post-traumatic stress disorder: randomised controlled trial. Br J Psychiatry 2012, 200:224-231.

897. Resick PA, Nishith P, Weaver TL, Astin MC, Feuer CA: A comparison of cognitive-processing therapy with prolonged exposure and a waiting condition for the treatment of chronic posttraumatic stress disorder in female rape victims. J Consult Clin Psychol 2002, 70:867-879.

898. Foa EB, Dancu CV, Hembree EA, Jaycox LH, Meadows EA, Street GP: A comparison of exposure therapy, stress inoculation training, and their combination for reducing posttraumatic stress disorder in female assault victims. J Consult Clin Psychol 1999, 67:194-200.

899. Resick PA, Galovski TE, O'Brien Uhlmansiek M, Scher CD, Clum GA, Young$X u Y$ : A randomized clinical trial to dismantle components of cognitive processing therapy for posttraumatic stress disorder in female victims of interpersonal violence. J Consult Clin Psychol 2008, 76:243-258.

900. Galovski TE, Monson C, Bruce SE, Resick PA: Does cognitive-behavioral therapy for PTSD improve perceived health and sleep impairment? J Trauma Stress 2009, 22:197-204.

901. Resick PA, Williams LF, Suvak MK, Monson CM, Gradus JL: Long-term outcomes of cognitive-behavioral treatments for posttraumatic stress disorder among female rape survivors. I Consult Clin Psychol 2012, 80:201-210.

902. Bryant RA, Moulds ML, Guthrie RM, Dang ST, Nixon RD: Imaginal exposure alone and imaginal exposure with cognitive restructuring in treatment of posttraumatic stress disorder. J Consult Clin Psychol 2003, 71:706-712.

903. Ehlers A, Clark D, Hackmann A, McManus F, Fennell M, Herbert C, Mayou R: A randomized controlled trial of cognitive therapy, a self-help booklet, and repeated assessments as early interventions for posttraumatic stress disorder. Arch Gen Psychiatry 2003, 60:1024-1032.

904. Zoellner T, Rabe S, Karl A, Maercker A: Post-traumatic growth as outcome of a cognitive-behavioural therapy trial for motor vehicle accident survivors with PTSD. Psychol Psychother 2011, 84:201-213. 
905. Kemp M, Drummond $\mathrm{P}, \mathrm{McDermott} B$ : A wait-list controlled pilot study of eye movement desensitization and reprocessing (EMDR) for children with post-traumatic stress disorder (PTSD) symptoms from motor vehicle accidents. Clinical child psychology and psychiatry 2010, 15:5-25.

906. Beck JG, Coffey SF, Foy DW, Keane TM, Blanchard EB: Group cognitive behavior therapy for chronic posttraumatic stress disorder: an initial randomized pilot study. Behav Ther 2009, 40:82-92.

907. Basoglu M, Salcioglu E, Livanou M: A randomized controlled study of single-session behavioural treatment of earthquake-related posttraumatic stress disorder using an earthquake simulator. Psychol Med 2007, 37:203-213.

908. Hogberg G, Pagani M, Sundin O, Soares J, Aberg-Wistedt A, Tarnell B, Hallstrom T: On treatment with eye movement desensitization and reprocessing of chronic post-traumatic stress disorder in public transportation workers-a randomized controlled trial. Nord J Psychiatry 2007, 61:54-61.

909. Keane TM, Zimering RT, Caddell JM: Implosive (flooding) therapy reduces symptoms of PTSD in Vietnam combat veterans. Behav Ther 1989, 20:245-260

910. Forbes $D$, Lloyd D, Nixon RD, Elliott P, Varker T, Perry D, Bryant RA, Creamer M: A multisite randomized controlled effectiveness trial of cognitive processing therapy for military-related posttraumatic stress disorder. J Anxiety Disord 2012, 26:442-452.

911. Alvarez J, McLean C, Harris AH, Rosen CS, Ruzek Jl, Kimerling R: The comparative effectiveness of cognitive processing therapy for male veterans treated in a VHA posttraumatic stress disorder residential rehabilitation program. J Consult Clin Psychol 2011, 79:590-599.

912. Macdonald A, Monson CM, Doron-Lamarca S, Resick PA, Palfai TP: Identifying patterns of symptom change during a randomized controlled trial of cognitive processing therapy for military-related posttraumatic stress disorder. J Trauma Stress 2011, 24:268-276.

913. Monson CM, Schnurr PP, Resick PA, Friedman MJ, Young-Xu Y, Stevens SP: Cognitive processing therapy for veterans with military-related posttraumatic stress disorder. J Consult Clin Psychol 2006, 74:898-907.

914. Gros DF, Yoder M, Tuerk PW, Lozano BE, Acierno R: Exposure therapy for PTSD delivered to veterans via telehealth: predictors of treatment completion and outcome and comparison to treatment delivered in person. Behav Ther 2011, 42:276-283.

915. van Minnen A, Foa EB: The effect of imaginal exposure length on outcome of treatment for PTSD. J Trauma Stress 2006, 19:427-438.

916. Bryant RA, Moulds ML, Guthrie RM, Dang ST, Mastrodomenico J, Nixon RD, Felmingham KL, Hopwood S, Creamer M: A randomized controlled trial of exposure therapy and cognitive restructuring for posttraumatic stress disorder. J Consult Clin Psychol 2008, 76:695-703.

917. Foa EB, Hembree EA, Cahill SP, Rauch SA, Riggs DS, Feeny NC, Yadin E: Randomized trial of prolonged exposure for posttraumatic stress disorder with and without cognitive restructuring: outcome at academic and community clinics. J Consult Clin Psychol 2005, 73:953-964.

918. Paunovic N, Öst L-G: Cognitive-behavior therapy vs exposure therapy in the treatment of PTSD in refugees. Behav Res Ther 2001, 39:1183-1197.

919. Moser JS, Cahill SP, Foa EB: Evidence for poorer outcome in patients with severe negative trauma-related cognitions receiving prolonged exposure plus cognitive restructuring: implications for treatment matching in posttraumatic stress disorder. J Nerv Ment Dis 2010, 198:72-75.

920. Grunert BK, Weis JM, Smucker MR, Christianson HF: Imagery rescripting and reprocessing therapy after failed prolonged exposure for posttraumatic stress disorder following industrial injury. I Behav Ther Exp Psychiatry 2007, 38:317-328.

921. Arntz A, Tiesema M, Kindt M: Treatment of PTSD: a comparison of imaginal exposure with and without imagery rescripting. J Behav Ther Exp Psychiatry 2007, 38:345-370.

922. Beidel DC, Frueh BC, Unde TW, Wong N, Mentrikoski JM: Multicomponent behavioral treatment for chronic combat-related posttraumatic stress disorder: a randomized controlled trial. J Anxiety Disord 2011, 25:224-231.

923. Bradley R, Greene J, Russ E, Dutra L, Westen D: A multidimensional metaanalysis of psychotherapy for PTSD. Am J Psychiatry 2005, 162:214-227.

924. Kar N: Cognitive behavioral therapy for the treatment of post-traumatic stress disorder: a review. Neuropsychiatr Dis Treat 2011, 7:167-181.
925. Schottenbauer MA, Glass CR, Arnkoff DB, Tendick V, Gray SH: Nonresponse and dropout rates in outcome studies on PTSD: review and methodological considerations. Psychiatry 2008, 71:134-168.

926. van der Kolk BA, Roth S, Pelcovitz D, Sunday S, Spinazzola J: Disorders of extreme stress: The empirical foundation of a complex adaptation to trauma. J Trauma Stress 2005, 18:389-399.

927. Najavits LM: Expanding the boundaries of PTSD treatment. JAMA 2012, 308:714-716.

928. Steil R, Dyer A, Priebe K, Kleindienst N, Bohus M: Dialectical behavior therapy for posttraumatic stress disorder related to childhood sexual abuse: a pilot study of an intensive residential treatment program. J Trauma Stress 2011, 24:102-106

929. Harned MS, Jackson SC, Comtois KA, Linehan MM: Dialectical behavior therapy as a precursor to PTSD treatment for suicidal and/or selfinjuring women with borderline personality disorder. $J$ Trauma Stress 2010, 23:421-429.

930. Harned MS, Korslund KE, Foa EB, Linehan MM: Treating PTSD in suicidal and self-injuring women with borderline personality disorder: development and preliminary evaluation of a Dialectical Behavior Therapy Prolonged Exposure Protocol. Behav Res Ther 2012, 50:381-386.

931. Mills KL, Teesson M, Back SE, Brady KT, Baker AL, Hopwood S, Sannibale C, Barrett EL, Merz S, Rosenfeld J, Ewer PL: Integrated exposure-based therapy for co-occurring posttraumatic stress disorder and substance dependence: a randomized controlled trial. JAMA 2012, 308:690-699.

932. Cloitre M, Courtois CA, Charuvastra A, Carapezza R, Stolbach BC, Green BL: Treatment of complex PTSD: results of the ISTSS expert clinician survey on best practices. J Trauma Stress 2011, 24:615-627.

933. Brunet A, Ashbaugh A, Hebert P: Internet use in the aftermath of trauma. Amsterdam: NATO Books, IOS Press; 2010

934. Spence J, Titov N, Dear BF, Johnston L, Solley K, Lorian C, Wootton B, Zou J, Schwenke G: Randomized controlled trial of Internet-delivered cognitive behavioral therapy for posttraumatic stress disorder. Depress Anxiety 2011, 28:541-550.

935. Litz BT, Engel CC, Bryant RA, Papa A: A randomized, controlled proof-ofconcept trial of an internet-based, therapist-assisted self-management treatment for posttraumatic stress disorder. Am J Psychiatry 2007 164:1676-1683

936. Knaevelsrud C, Maercker A: Internet-based treatment for PTSD reduces distress and facilitates the development of a strong therapeutic alliance: a randomized controlled clinical trial. BMC Psychiatry 2007, 7:13.

937. Klein B, Mitchell J, Abbott J, Shandley K, Austin D, Gilson K, Kiropoulos L, Cannard G, Redman T: A therapist-assisted cognitive behavior therapy internet intervention for posttraumatic stress disorder: pre-, post- and 3-month follow-up results from an open trial. J Anxiety Disord 2010, 24:635-644.

938. Knaevelsrud C, Liedl A, Maercker A: Posttraumatic growth, optimism and openness as outcomes of a cognitive-behavioural intervention for posttraumatic stress reactions. J Health Psychol 2010, 15:1030-1038.

939. Lange A, van de Ven JP, Schrieken B, Emmelkamp PM: Interapy, treatment of posttraumatic stress through the Internet: a controlled trial. J Behav Ther Exp Psychiatry 2001, 32:73-90.

940. Lange A, Rietdijk D, Hudcovicova M, van de Ven JP, Schrieken B, Emmelkamp PM: Interapy: a controlled randomized trial of the standardized treatment of posttraumatic stress through the internet. J Consult Clin Psychol 2003, 71:901-909.

941. Rothbaum BO, Hodges LF, Ready D, Graap K, Alarcon RD: Virtual reality exposure therapy for Vietnam veterans with posttraumatic stress disorder. J Clin Psychiatry 2001, 62:617-622.

942. Ready DJ, Gerardi RJ, Backscheider AG, Mascaro N, Rothbaum BO: Comparing virtual reality exposure therapy to present-centered therapy with 11 U.S. Vietnam veterans with PTSD. Cyberpsychol Behav Soc Netw 2010, 13:49-54.

943. Difede J, Cukor J, Jayasinghe N, Patt I, Jedel S, Spielman L, Giosan C, Hoffman HG: Virtual reality exposure therapy for the treatment of posttraumatic stress disorder following September 11, 2001. J Clin Psychiatry 2007, 68:1639-1647.

944. Germain V, Marchand A, Bouchard S, Drouin MS, Guay S: Effectiveness of cognitive behavioural therapy administered by videoconference for posttraumatic stress disorder. Cogn Behav Ther 2009, 38:42-53. 
945. Hetrick SE, Purcell R, Garner B, Parslow R: Combined pharmacotherapy and psychological therapies for post traumatic stress disorder (PTSD). Cochrane Database Syst Rev 2010, CD007316.

946. Cohen JA, Mannarino AP, Perel JM, Staron V: A pilot randomized controlled trial of combined trauma-focused CBT and sertraline for childhood PTSD symptoms. J Am Acad Child Adolesc Psychiatry 2007, 46:811-819.

947. Simon NM, Connor KM, Lang AJ, Rauch S, Krulewicz S, LeBeau RT, Davidson JR, Stein MB, Otto MW, Foa EB, Pollack MH: Paroxetine CR augmentation for posttraumatic stress disorder refractory to prolonged exposure therapy. J Clin Psychiatry 2008, 69:400-405.

948. Rothbaum BO, Cahill SP, Foa EB, Davidson JR, Compton J, Connor KM, Astin MC, Hahn CG: Augmentation of sertraline with prolonged exposure in the treatment of posttraumatic stress disorder. J Trauma Stress 2006, 19:625-638

949. Otto M, Hinton D, Korbly N, Chea A, Ba P, Gershuny B, Pollack M: Treatment of pharmacotherapy-refractory posttraumatic stress disorder among Cambodian refugees: a pilot study of combination treatment with cognitive-behavior therapy vs sertraline alone. Behav Res Ther 2003, 41:1271-1276

950. Schneier FR, Neria Y, Pavlicova M, Hembree E, Suh EJ, Amsel L, Marshall RD: Combined prolonged exposure therapy and paroxetine for PTSD related to the World Trade Center attack: a randomized controlled trial. Am J Psychiatry 2012, 169:80-88.

951. Brunet A, Orr SP, Tremblay J, Robertson K, Nader K, Pitman RK: Effect of post-retrieval propranolol on psychophysiologic responding during subsequent script-driven traumatic imagery in post-traumatic stress disorder. J Psychiatr Res 2008, 42:503-506.

952. Brunet A, Poundja J, Tremblay J, Bui E, Thomas E, Orr SP, Azzoug A, Birmes $P$, Pitman RK: Trauma reactivation under the influence of propranolol decreases posttraumatic stress symptoms and disorder: 3 open-label trials. J Clin Psychopharmacol 2011, 31:547-550

953. de Kleine RA, Hendriks GJ, Kusters WJ, Broekman TG, van Minnen A: A randomized placebo-controlled trial of D-cycloserine to enhance exposure therapy for posttraumatic stress disorder. Biol Psychiatry 2012, 71:962-968.

954. Litz BT, Salters-Pedneault K, Steenkamp MM, Hermos JA, Bryant RA, Otto MW, Hofmann SG: A randomized placebo-controlled trial of d-cycloserine and exposure therapy for posttraumatic stress disorder. J Psychiatr Res 2012, 46:1184-1190.

955. Van Etten M, Taylor S: Comparative efficacy of treatments for posttraumatic stress disorder: a meta-analysis. Clin Psychol Psychother 1998, 5:126-144.

956. Edmond T, Rubin A: Assessing the long-term effects of EMDR: results from an 18-month follow-up study with adult female survivors of CSA. J Child Sex Abus 2004, 13:69-86.

957. Wilson SA, Becker LA, Tinker RH: Fifteen-month follow-up of eye movement desensitization and reprocessing (EMDR) treatment for posttraumatic stress disorder and psychological trauma. J Consult Clin Psychol 1997, 65:1047-1056.

958. Knaevelsrud C, Maercker A: Long-term effects of an internet-based treatment for posttraumatic stress. Cogn Behav Ther 2010, 39:72-77.

959. Hogberg G, Pagani M, Sundin O, Soares J, Aberg-Wistedt A, Tarnell B, Hallstrom T: Treatment of post-traumatic stress disorder with eye movement desensitization and reprocessing: outcome is stable in 35month follow-up. Psychiatry Res 2008, 159:101-108.

960. van der Kolk B, Dreyfuss D, Michaels M, Shera D, Berkowitz R, Fisler R, Saxe G: Fluoxetine in posttraumatic stress disorder. J Clin Psychiatry 1994 55:517-522.

961. Connor K, Sutherland S, Tupler L, Malik M, Davidson J: Fluoxetine in posttraumatic stress disorder. Randomised, double-blind study. $\mathrm{Br} J$ Psychiatry 1999, 175:17-22

962. Martenyi F, Brown E, Zhang H, Prakash A, Koke S: Fluoxetine versus placebo in posttraumatic stress disorder. J Clin Psychiatry 2002, 63:199-206.

963. van der Kolk BA, Spinazzola J, Blaustein ME, Hopper JW, Hopper EK, Korn DL, Simpson WB: A randomized clinical trial of eye movement desensitization and reprocessing (EMDR), fluoxetine, and pill placebo in the treatment of posttraumatic stress disorder: treatment effects and long-term maintenance. J Clin Psychiatry 2007, 68:37-46.

964. Martenyi F, Brown EB, Caldwell CD: Failed efficacy of fluoxetine in the treatment of posttraumatic stress disorder: results of a fixed-dose, placebo-controlled study. J Clin Psychopharmacol 2007, 27:166-170.
965. Hertzberg MA, Feldman ME, Beckham JC, Kudler HS, Davidson JR: Lack of efficacy for fluoxetine in PTSD: a placebo controlled trial in combat veterans. Ann Clin Psychiatry 2000, 12:101-105.

966. Tucker P, Zaninelli R, Yehuda R, Ruggiero L, Dillingham K, Pitts C: Paroxetine in the treatment of chronic posttraumatic stress disorder: results of a placebo-controlled, flexible-dosage trial. J Clin Psychiatry 2001, 62:860-868.

967. Marshall R, Beebe K, Oldham M, Zaninelli R: Efficacy and safety of paroxetine treatment for chronic PTSD: a fixed-dose, placebo-controlled study. Am J Psychiatry 2001, 158:1982-1988.

968. Stein D, Davidson J, Seedat S, Beebe K: Paroxetine in the treatment of post-traumatic stress disorder: pooled analysis of placebo-controlled studies. Expert Opin Pharmacother 2003, 4:1829-1838.

969. Marshall RD, Lewis-Fernandez R, Blanco C, Simpson HB, Lin SH, Vermes D, Garcia W, Schneier F, Neria Y, Sanchez-Lacay A, Liebowitz MR: A controlled trial of paroxetine for chronic PTSD, dissociation, and interpersonal problems in mostly minority adults. Depress Anxiety 2007, 24:77-84.

970. Petrakis IL, Ralevski E, Desai N, Trevisan L, Gueorguieva R, Rounsaville B, Krystal JH: Noradrenergic vs serotonergic antidepressant with or without naltrexone for veterans with PTSD and comorbid alcohol dependence. Neuropsychopharmacology 2012, 37:996-1004.

971. Davidson J, Rothbaum B, van der Kolk B, Sikes C, Farfel G: Multicenter, double-blind comparison of sertraline and placebo in the treatment of posttraumatic stress disorder. Arch Gen Psychiatry 2001, 58:485-492.

972. Brady K, Pearlstein T, Asnis G, Baker D, Rothbaum B, Sikes C, Farfel G: Efficacy and safety of sertraline treatment of posttraumatic stress disorder: a randomized controlled trial. JAMA 2000, 283:1837-1844.

973. Zohar J, Amital D, Miodownik C, Kotler M, Bleich A, Lane R, Austin C: Double-blind placebo-controlled pilot study of sertraline in military veterans with posttraumatic stress disorder. J Clin Psychopharmacol 2002, 22:190-195.

974. Tucker P, Potter-Kimball R, Wyatt D, Parker D, Burgin C, Jones D, Masters B: Can physiologic assessment and side effects tease out differences in PTSD trials? A double-blind comparison of citalopram, sertraline, and placebo. Psychopharmacol Bull 2003, 37:135-149.

975. Davidson J, Rothbaum BO, Tucker P, Asnis G, Benattia I, Musgnung JJ: Venlafaxine extended release in posttraumatic stress disorder: a sertraline- and placebo-controlled study. J Clin Psychopharmacol 2006 26:259-267.

976. Stein DJ, van der Kolk BA, Austin C, Fayyad R, Clary C: Efficacy of sertraline in posttraumatic stress disorder secondary to interpersonal trauma or childhood abuse. Ann Clin Psychiatry 2006, 18:243-249.

977. Friedman MJ, Marmar CR, Baker DG, Sikes CR, Farfel GM: Randomized, double-blind comparison of sertraline and placebo for posttraumatic stress disorder in a Department of Veterans Affairs setting. J Clin Psychiatry 2007, 68:711-720.

978. Panahi Y, Moghaddam BR, Sahebkar A, Nazari MA, Beiraghdar F, Karami G, Saadat AR: A randomized, double-blind, placebo-controlled trial on the efficacy and tolerability of sertraline in Iranian veterans with posttraumatic stress disorder. Psychol Med 2011, 41:2159-2166.

979. Marmar C, Schoenfeld F, Weiss D, Metzler T, Zatzick D, Wu R, Smiga S, Tecott L, Neylan T: Open trial of fluvoxamine treatment for combatrelated posttraumatic stress disorder. J Clin Psychiatry 1996, 57(Suppl 8):66-70, discussion 71-62.

980. Escalona R, Canive J, Calais L, Davidson J: Fluvoxamine treatment in veterans with combat-related post-traumatic stress disorder. Depress Anxiety 2002, 15:29-33.

981. Tucker P, Smith K, Marx B, Jones D, Miranda R, Lensgraf J: Fluvoxamine reduces physiologic reactivity to trauma scripts in posttraumatic stress disorder. J Clin Psychopharmacol 2000, 20:367-372.

982. Neylan T, Metzler T, Schoenfeld F, Weiss D, Lenoci M, Best S, Lipsey T, Marmar C: Fluvoxamine and sleep disturbances in posttraumatic stress disorder. J Trauma Stress 2001, 14:461-467.

983. De Boer M, Op den Velde W, Falger P, Hovens J, De Groen J, Van Duijn H: Fluvoxamine treatment for chronic PTSD: a pilot study. Psychother Psychosom 1992, 57:158-163

984. Spivak B, Strous RD, Shaked G, Shabash E, Kotler M, Weizman A: Reboxetine versus fluvoxamine in the treatment of motor vehicle accident-related posttraumatic stress disorder: a double-blind, fixeddosage, controlled trial. J Clin Psychopharmacol 2006, 26:152-156. 
985. Robert S, Hamner MB, Ulmer HG, Lorberbaum JP, Durkalski VL: Open-label trial of escitalopram in the treatment of posttraumatic stress disorder. $J$ Clin Psychiatry 2006, 67:1522-1526.

986. Seedat S, Stein D, Emsley R: Open trial of citalopram in adults with posttraumatic stress disorder. Int I Neuropsychopharmacol 2000, 3:135-140.

987. Seedat S, Stein D, Ziervogel C, Middleton T, Kaminer D, Emsley R, Rossouw W: Comparison of response to a selective serotonin reuptake inhibitor in children, adolescents, and adults with posttraumatic stress disorder. J Child Adolesc Psychopharmacol 2002, 12:37-46.

988. English BA, Jewell M, Jewell G, Ambrose S, Davis LL: Treatment of chronic posttraumatic stress disorder in combat veterans with citalopram: an open trial. J Clin Psychopharmacol 2006, 26:84-88.

989. Davidson J, Baldwin D, Stein DJ, Kuper E, Benattia I, Ahmed S, Pedersen R, Musgnung J: Treatment of posttraumatic stress disorder with venlafaxine extended release: a 6-month randomized controlled trial. Arch Gen Psychiatry 2006, 63:1158-1165.

990. Walderhaug E, Kasserman S, Aikins D, Vojvoda D, Nishimura C, Neumeister A: Effects of duloxetine in treatment-refractory men with posttraumatic stress disorder. Pharmacopsychiatry 2010, 43:45-49.

991. Villarreal G, Canive JM, Calais LA, Toney G, Smith AK: Duloxetine in military posttraumatic stress disorder. Psychopharmacol Bull 2010, 43:26-34.

992. Frank J, Kosten T, Giller E, Dan E: A randomized clinical trial of phenelzine and imipramine for posttraumatic stress disorder. Am J Psychiatry 1988, 145:1289-1291

993. Kosten T, Frank J, Dan E, McDougle C, Giller E: Pharmacotherapy for posttraumatic stress disorder using phenelzine or imipramine. J Nerv Ment Dis 1991, 179:366-370.

994. Davidson J, Kudler H, Smith R, Mahorney S, Lipper S, Hammett E, Saunders W, Cavenar J: Treatment of posttraumatic stress disorder with amitriptyline and placebo. Arch Gen Psychiatry 1990, 47:259-266.

995. Reist C, Kauffmann C, Haier R, Sangdahl C, De ME, Chicz-De MA, Nelson J. A controlled trial of desipramine in 18 men with posttraumatic stress disorder. Am J Psychiatry 1989, 146:513-516.

996. Shestatzky M, Greenberg D, Lerer B: A controlled trial of phenelzine in posttraumatic stress disorder. Psychiatry Res 1988, 24:149-155.

997. Onder $E$, Tural U, Aker T: A comparative study of fluoxetine, moclobemide, and tianeptine in the treatment of posttraumatic stress disorder following an earthquake. Eur Psychiatry 2006, 21:174-179.

998. Neal L, Shapland W, Fox C: An open trial of moclobemide in the treatment of post-traumatic stress disorder. Int Clin Psychopharmacol 1997, 12:231-237.

999. Connor K, Davidson J, Weisler R, Ahearn E: A pilot study of mirtazapine in post-traumatic stress disorder. Int Clin Psychopharmacol 1999, 14:29-31.

1000. Davidson JR, Weisler RH, Butterfield Ml, Casat CD, Connor KM, Barnett S, van Meter S: Mirtazapine vs. placebo in posttraumatic stress disorder: a pilot trial. Biol Psychiatry 2003, 53:188-191.

1001. Chung M, Min K, Jun Y, Kim S, Kim W, Jun E: Efficacy and tolerability of mirtazapine and sertraline in Korean veterans with posttraumatic stress disorder: a randomized open label trial. Hum Psychopharmacol 2004, 19:489-494.

1002. Alderman CP, Condon JT, Gilbert AL: An open-label study of mirtazapine as treatment for combat-related PTSD. Ann Pharmacother 2009, 43:1220-1226.

1003. Canive J, Clark R, Calais L, Qualls C, Tuason V: Bupropion treatment in veterans with posttraumatic stress disorder: an open study. J Clin Psychopharmacol 1998, 18:379-383

1004. Franciskovic T, Sukovic Z, Janovic S, Stevanovic A, Nemcic-Moro I, Roncevic-Grzeta I, Letica-Crepulja M: Tianeptine in the combined treatment of combat related posttraumatic stress disorder. Psychiatr Danub 2011, 23:257-263.

1005. Becker ME, Hertzberg MA, Moore SD, Dennis MF, Bukenya DS, Beckham JC: A placebo-controlled trial of bupropion SR in the treatment of chronic posttraumatic stress disorder. J Clin Psychopharmacol 2007, 27:193-197.

1006. Braun P, Greenberg D, Dasberg H, Lerer B: Core symptoms of posttraumatic stress disorder unimproved by alprazolam treatment. J Clin Psychiatry 1990, 51:236-238.

1007. Cates M, Bishop M, Davis L, Lowe J, Woolley T: Clonazepam for treatment of sleep disturbances associated with combat-related posttraumatic stress disorder. Ann Pharmacother 2004, 38:1395-1399.
1008. Shalev A, Rogel-Fuchs Y: Auditory startle reflex in post-traumatic stress disorder patients treated with clonazepam. Isr J Psychiatry Relat Sci 1992, 29:1-6.

1009. Tucker P, Trautman RP, Wyatt DB, Thompson J, Wu SC, Capece JA, Rosenthal NR: Efficacy and safety of topiramate monotherapy in civilian posttraumatic stress disorder: a randomized, double-blind, placebocontrolled study. J Clin Psychiatry 2007, 68:201-206.

1010. Yeh MS, Mari JJ, Costa MC, Andreoli SB, Bressan RA, Mello MF: A doubleblind randomized controlled trial to study the efficacy of topiramate in a civilian sample of PTSD. CNS Neurosci Ther 2011, 17:305-310.

1011. Hertzberg M, Butterfield M, Feldman M, Beckham J, Sutherland S, Connor K, Davidson J: A preliminary study of lamotrigine for the treatment of posttraumatic stress disorder. Biol Psychiatry 1999, 45:1226-1229.

1012. Lipper S, Davidson J, Grady T, Edinger J, Hammett E, Mahorney S, Cavenar J: Preliminary study of carbamazepine in post-traumatic stress disorder. Psychosomatics 1986, 27:849-854.

1013. Wolf M, Alavi A, Mosnaim A: Posttraumatic stress disorder in Vietnam veterans clinical and EEG findings; possible therapeutic effects of carbamazepine. Biol Psychiatry 1988, 23:642-644.

1014. Fesler F: Valproate in combat-related posttraumatic stress disorder. $J$ Clin Psychiatry 1991, 52:361-364.

1015. Clark R, Canive J, Calais L, Qualls C, Tuason V: Divalproex in posttraumatic stress disorder: an open-label clinical trial. J Trauma Stress 1999, 12:395-401.

1016. Davis LL, Davidson JR, Ward LC, Bartolucci A, Bowden CL, Petty F: Divalproex in the treatment of posttraumatic stress disorder: a randomized, double-blind, placebo-controlled trial in a veteran population. J Clin Psychopharmacol 2008, 28:84-88.

1017. Hamner MB, Faldowski RA, Robert S, Ulmer HG, Horner MD, Lorberbaum JP: A preliminary controlled trial of divalproex in posttraumatic stress disorder. Ann Clin Psychiatry 2009, 21:89-94.

1018. Davidson JR, Brady K, Mellman TA, Stein MB, Pollack MH: The efficacy and tolerability of tiagabine in adult patients with post-traumatic stress disorder. J Clin Psychopharmacol 2007, 27:85-88.

1019. Hamner M, Brodrick P, Labbate L: Gabapentin in PTSD: a retrospective, clinical series of adjunctive therapy. Ann Clin Psychiatry 2001, 13:141-146.

1020. Malek-Ahmadi P: Gabapentin and posttraumatic stress disorder. Ann Pharmacother 2003, 37:664-666.

1021. Kinrys G, Wygant LE, Pardo TB, Melo M: Levetiracetam for treatmentrefractory posttraumatic stress disorder. J Clin Psychiatry 2006, 67:211-214.

1022. Pae CU, Marks DM, Han C, Masand PS, Patkar AA: Pregabalin augmentation of antidepressants in patients with accident-related posttraumatic stress disorder: an open label pilot study. Int Clin Psychopharmacol 2009, 24:29-33.

1023. Schwartz T: The use of tiagabine augmentation for treatment-resistant anxiety disorders: a case series. Psychopharmacol Bull 2002, 36:53-57.

1024. Taylor F: Tiagabine for posttraumatic stress disorder: a case series of 7 women. J Clin Psychiatry 2003, 64:1421-1425.

1025. Berigan T: Treatment of posttraumatic stress disorder with tiagabine. Can J Psychiatry 2002, 47:788.

1026. Berlant J: Prospective open-label study of add-on and monotherapy topiramate in civilians with chronic nonhallucinatory posttraumatic stress disorder. BMC Psychiatry 2004, 4:24.

1027. Berlant J, van Kammen D: Open-label topiramate as primary or adjunctive therapy in chronic civilian posttraumatic stress disorder: a preliminary report. J Clin Psychiatry 2002, 63:15-20.

1028. Lindley SE, Carlson EB, Hill K: A randomized, double-blind, placebocontrolled trial of augmentation topiramate for chronic combat-related posttraumatic stress disorder. J Clin Psychopharmacol 2007, 27:677-681.

1029. Alderman CP, McCarthy LC, Condon JT, Marwood AC, Fuller JR: Topiramate in combat-related posttraumatic stress disorder. Ann Pharmacother 2009, 43:635-641.

1030. Padala PR, Madison J, Monnahan M, Marcil W, Price P, Ramaswamy S, Din AU, Wilson DR, Petty F: Risperidone monotherapy for post-traumatic stress disorder related to sexual assault and domestic abuse in women. Int Clin Psychopharmacol 2006, 21:275-280.

1031. Villarreal G, Calais LA, Canive JM, Lundy SL, Pickard J, Toney G: Prospective study to evaluate the efficacy of aripiprazole as a monotherapy in 
patients with severe chronic posttraumatic stress disorder: an open trial. Psychopharmacol Bull 2007, 40:6-18.

1032. Mello MF, Costa MC, Schoedl AF, Fiks JP: Aripiprazole in the treatment of posttraumatic stress disorder: an open-label trial. Rev Bras Psiquiatr 2008, 30:358-361

1033. Lambert MT: Aripiprazole in the management of post-traumatic stress disorder symptoms in returning global war on terrorism veterans. Int Clin Psychopharmacol 2006, 21:185-187.

1034. Kozaric-Kovacic D, Pivac N: Quetiapine treatment in an open trial in combat-related post-traumatic stress disorder with psychotic features. Int I Neuropsychopharmacol 2007, 10:253-261.

1035. Byers MG, Allison KM, Wendel CS, Lee JK: Prazosin versus quetiapine for nighttime posttraumatic stress disorder symptoms in veterans: an assessment of long-term comparative effectiveness and safety. J Clin Psychopharmacol 2010, 30:225-229.

1036. Butterfield Ml, Becker ME, Connor KM, Sutherland S, Churchill LE, Davidson JR: Olanzapine in the treatment of post-traumatic stress disorder: a pilot study. Int Clin Psychopharmacol 2001, 16:197-203.

1037. Pivac N, Kozaric-Kovacic D, Muck-Seler D: Olanzapine versus fluphenazine in an open trial in patients with psychotic combat-related posttraumatic stress disorder. Psychopharmacology (Berl) 2004, 175:451-456.

1038. Petty F, Brannan S, Casada J, Davis L, Gajewski V, Kramer G, Stone R, Teten A, Worchel J, Young K: Olanzapine treatment for post-traumatic stress disorder: an open-label study. Int Clin Psychopharmacol 2001, 16:331-337.

1039. Hamner M, Faldowski R, Ulmer H, Frueh B, Huber M, Arana G: Adjunctive risperidone treatment in post-traumatic stress disorder: a preliminary controlled trial of effects on comorbid psychotic symptoms. Int Clin Psychopharmacol 2003, 18:1-8.

1040. Monnelly E, Ciraulo D, Knapp C, Keane T: Low-dose risperidone as adjunctive therapy for irritable aggression in posttraumatic stress disorder. J Clin Psychopharmacol 2003, 23:193-196.

1041. Bartzokis G, Lu P, Turner J, Mintz J, Saunders C: Adjunctive risperidone in the treatment of chronic combat-related posttraumatic stress disorder. Biol Psychiatry 2005, 57:474-479.

1042. Reich D, Winternitz S, Hennen J, Watts T, Stanculescu C: A preliminary study of risperidone in the treatment of posttraumatic stress disorder related to childhood abuse in women. J Clin Psychiatry 2004, 65:1601-1606

1043. Krystal JH, Rosenheck RA, Cramer JA, Vessicchio JC, Jones KM, Vertrees JE, Horney RA, Huang GD, Stock C: Adjunctive risperidone treatment for antidepressant-resistant symptoms of chronic military service-related PTSD: a randomized trial. JAMA 2011, 306:493-502.

1044. Rothbaum BO, Killeen TK, Davidson JR, Brady KT, Connor KM, Heekin MH: Placebo-controlled trial of risperidone augmentation for selective serotonin reuptake inhibitor-resistant civilian posttraumatic stress disorder. J Clin Psychiatry 2008, 69:520-525.

1045. Stein M, Kline N, Matloff J: Adjunctive olanzapine for SSRI-resistant combat-related PTSD: A double-blind, placebo-controlled study. Am J Psychiatry 2002, 159:1777-1779.

1046. Robert S, Hamner MB, Durkalski VL, Brown MW, Ulmer HG: An open-label assessment of aripiprazole in the treatment of PTSD. Psychopharmacol Bull 2009, 42:69-80.

1047. Richardson JD, Fikretoglu D, Liu A, Mclntosh D: Aripiprazole augmentation in the treatment of military-related PTSD with major depression: a retrospective chart review. BMC Psychiatry 2011, 11:86.

1048. Sokolski K, Denson T, Lee R, Reist C: Quetiapine for treatment of refractory symptoms of combat-related post-traumatic stress disorder. Mil Med 2003, 168:486-489.

1049. Hamner M, Deitsch S, Brodrick P, Ulmer H, Lorberbaum J: Quetiapine treatment in patients with posttraumatic stress disorder: an open trial of adjunctive therapy. J Clin Psychopharmacol 2003, 23:15-20.

1050. Ahearn EP, Mussey M, Johnson C, Krohn A, Krahn D: Quetiapine as an adjunctive treatment for post-traumatic stress disorder: an 8-week open-label study. Int Clin Psychopharmacol 2006, 21:29-33.

1051. Duffy J, Malloy P: Efficacy of buspirone in the treatment of posttraumatic stress disorder: an open trial. Ann Clin Psychiatry 1994, 6:33-37.

1052. Wells B, Chu C, Johnson R, Nasdahl C, Ayubi M, Sewell E, Statham P: Buspirone in the treatment of posttraumatic stress disorder. Pharmacotherapy 1991, 11:340-343.
1053. Hertzberg M, Feldman M, Beckham J, Davidson J: Trial of trazodone for posttraumatic stress disorder using a multiple baseline group design. J Clin Psychopharmacol 1996, 16:294-298.

1054. Battista MA, Hierholzer R, Khouzam HR, Barlow A, OToole S: Pilot trial of memantine in the treatment of posttraumatic stress disorder. Psychiatry 2007, 70:167-174.

1055. Pollack MH, Hoge EA, Worthington JJ, Moshier SJ, Wechsler RS, Brandes M, Simon NM: Eszopiclone for the treatment of posttraumatic stress disorder and associated insomnia: a randomized, double-blind, placebo-controlled trial. J Clin Psychiatry 2011, 72:892-897.

1056. Kinzie J, Leung P: Clonidine in Cambodian patients with posttraumatic stress disorder. J Nerv Ment Dis 1989, 177:546-550.

1057. Neylan TC, Lenoci M, Samuelson KW, Metzler TJ, Henn-Haase C, Hierholzer RW, Lindley SE, Otte C, Schoenfeld FB, Yesavage JA, Marmar CR: No improvement of posttraumatic stress disorder symptoms with guanfacine treatment. Am J Psychiatry 2006, 163:2186-2188.

1058. Davis LL, Ward C, Rasmusson A, Newell JM, Frazier E, Southwick SM: A placebo-controlled trial of guanfacine for the treatment of posttraumatic stress disorder in veterans. Psychopharmacol Bull 2008, 41:8-18.

1059. Abramowitz EG, Barak Y, Ben-Avi I, Knobler HY: Hypnotherapy in the treatment of chronic combat-related PTSD patients suffering from insomnia: a randomized, zolpidem-controlled clinical trial. Int I Clin Exp Hypn 2008, 56:270-280.

1060. Stein D, Seedat $\mathrm{S}$, van dLG, Zungu-Dirwayi N: Selective serotonin reuptake inhibitors in the treatment of post-traumatic stress disorder: a meta-analysis of randomized controlled trials. Int Clin Psychopharmacol 2000, 15(Suppl 2):S31-39.

1061. Pae CU, Lim HK, Peindl K, Ajwani N, Serretti A, Patkar AA, Lee C: The atypical antipsychotics olanzapine and risperidone in the treatment of posttraumatic stress disorder: a meta-analysis of randomized, doubleblind, placebo-controlled clinical trials. Int Clin Psychopharmacol 2008, 23:1-8.

1062. Raskind M, Peskind E, Kanter E, Petrie E, Radant A, Thompson C, Dobie D, Hoff D, Rein R, Straits-Troster K, et al: Reduction of nightmares and other PTSD symptoms in combat veterans by prazosin: a placebo-controlled study. Am J Psychiatry 2003, 160:371-373.

1063. Peskind E, Bonner L, Hoff D, Raskind M: Prazosin reduces trauma-related nightmares in older men with chronic posttraumatic stress disorder. J Geriatr Psychiatry Neurol 2003, 16:165-171.

1064. Taylor F, Raskind M: The alpha1-adrenergic antagonist prazosin improves sleep and nightmares in civilian trauma posttraumatic stress disorder. J Clin Psychopharmacol 2002, 22:82-85.

1065. Raskind MA, Peskind ER, Hoff DJ, Hart KL, Holmes HA, Warren D, Shofer J, O'Connell J, Taylor F, Gross C, et al: A parallel group placebo controlled study of prazosin for trauma nightmares and sleep disturbance in combat veterans with post-traumatic stress disorder. Biol Psychiatry 2007, 61:928-934.

1066. Taylor FB, Martin P, Thompson C, Williams J, Mellman TA, Gross C, Peskind ER, Raskind MA: Prazosin effects on objective sleep measures and clinical symptoms in civilian trauma posttraumatic stress disorder: a placebo-controlled study. Biol Psychiatry 2008, 63:629-632.

1067. Lubin G, Weizman A, Shmushkevitz M, Valevski A: Short-term treatment of post-traumatic stress disorder with naltrexone: an open-label preliminary study. Hum Psychopharmacol 2002, 17:181-185.

1068. Bohus MJ, Landwehrmeyer GB, Stiglmayr CE, Limberger MF, Bohme R, Schmahl CG: Naltrexone in the treatment of dissociative symptoms in patients with borderline personality disorder: an open-label trial. J Clin Psychiatry 1999, 60:598-603.

1069. Glover $\mathrm{H}$ : A preliminary trial of nalmefene for the treatment of emotional numbing in combat veterans with post-traumatic stress disorder. Isr J Psychiatry Relat Sci 1993, 30:255-263.

1070. Bills LJ, Kreisler K: Treatment of flashbacks with naltrexone. Am J Psychiatry 1993, 150:1430.

1071. Jacobs-Rebhun S, Schnurr PP, Friedman MJ, Peck R, Brophy M, Fuller D: Posttraumatic stress disorder and sleep difficulty [Letter]. Am J Psychiatry 2000, 157:1525-1526.

1072. Davidson J, Connor K, Hertzberg M, Weisler R, Wilson W, Payne V: Maintenance therapy with fluoxetine in posttraumatic stress disorder: a placebo-controlled discontinuation study. J Clin Psychopharmacol 2005, 25:166-169. 
1073. Martenyi F, Brown E, Zhang H, Koke S, Prakash A: Fluoxetine v. placebo in prevention of relapse in post-traumatic stress disorder. $\mathrm{Br} J$ Psychiatry 2002, 181:315-320.

1074. Davidson J, Pearlstein T, Londborg P, Brady K, Rothbaum B, Bell J, Maddock R, Hegel M, Farfel G: Efficacy of sertraline in preventing relapse of posttraumatic stress disorder: results of a 28-week double-blind, placebo-controlled study. Am J Psychiatry 2001, 158:1974-1981.

1075. Connor KM, Davidson JR, Weisler RH, Zhang W, Abraham K: Tiagabine for posttraumatic stress disorder: effects of open-label and double-blind discontinuation treatment. Psychopharmacology 2006, 184:21-25.

1076. Kim Y, Asukai N, Konishi T, Kato H, Hirotsune H, Maeda M, Inoue H, Narita $\mathrm{H}$, Iwasaki M: Clinical evaluation of paroxetine in post-traumatic stress disorder (PTSD): 52-week, non-comparative open-label study for clinical use experience. Psychiatry Clin Neurosci 2008, 62:646-652

1077. Londborg P, Hegel M, Goldstein S, Goldstein D, Himmelhoch J, Maddock R, Patterson W, Rausch J, Farfel G: Sertraline treatment of posttraumatic stress disorder: results of 24 weeks of open-label continuation treatment. J Clin Psychiatry 2001, 62:325-331.

1078. Boggio PS, Rocha M, Oliveira MO, Fecteau S, Cohen RB, Campanha C, Ferreira-Santos E, Meleiro A, Corchs F, Zaghi S, et al: Noninvasive brain stimulation with high-frequency and low-intensity repetitive transcranial magnetic stimulation treatment for posttraumatic stress disorder. J Clin Psychiatry 2010, 71:992-999.

1079. Watts BV, Landon B, Groft A, Young-Xu Y: A sham controlled study of repetitive transcranial magnetic stimulation for posttraumatic stress disorder. Brain Stimul 2012, 5:38-43.

1080. Osuch EA, Benson BE, Luckenbaugh DA, Geraci M, Post RM, McCann U: Repetitive TMS combined with exposure therapy for PTSD: a preliminary study. J Anxiety Disord 2009, 23:54-59.

1081. Margoob MA, Ali Z, Andrade C: Efficacy of ECT in chronic, severe, antidepressant- and CBT-refractory PTSD: an open, prospective study. Brain Stimul 2010, 3:28-35.

1082. Watts BV: Electroconvulsive therapy for comorbid major depressive disorder and posttraumatic stress disorder. J ECT 2007, 23:93-95.

1083. Hollifield M, Sinclair-Lian N, Warner TD, Hammerschlag R: Acupuncture for posttraumatic stress disorder: a randomized controlled pilot trial. J Nerv Ment Dis 2007, 195:504-513.

1084. Bormann JE, Thorp S, Wetherell JL, Golshan S: A spiritually based group intervention for combat veterans with posttraumatic stress disorder: feasibility study. J Holist Nurs 2008, 26:109-116.

1085. Rosenthal JZ, Grosswald S, Ross R, Rosenthal N: Effects of transcendental meditation in veterans of Operation Enduring Freedom and Operation Iraqi Freedom with posttraumatic stress disorder: a pilot study. Mil Med 2011, 176:626-630.

1086. Vesga-Lopez O, Blanco C, Keyes K, Olfson M, Grant BF, Hasin DS: Psychiatric disorders in pregnant and postpartum women in the United States. Arch Gen Psychiatry 2008, 65:805-815.

1087. Sutter-Dallay AL, Giaconne-Marcesche V, Glatigny-Dallay E, Verdoux H: Women with anxiety disorders during pregnancy are at increased risk of intense postnatal depressive symptoms: a prospective survey of the MATQUID cohort. Eur Psychiatry 2004, 19:459-463.

1088. Buist A, Gotman N, Yonkers KA: Generalized anxiety disorder: course and risk factors in pregnancy. J Affect Disord 2011, 131:277-283.

1089. Ross LE, McLean LM: Anxiety disorders during pregnancy and the postpartum period: A systematic review. I Clin Psychiatry 2006, 67:1285-1298

1090. Zambaldi CF, Cantilino A, Montenegro AC, Paes JA, de Albuquerque TL, Sougey EB: Postpartum obsessive-compulsive disorder: prevalence and clinical characteristics. Compr Psychiatry 2009, 50:503-509.

1091. Alcorn KL, O'Donovan A, Patrick JC, Creedy D, Devilly GJ: A prospective longitudinal study of the prevalence of post-traumatic stress disorder resulting from childbirth events. Psychol Med 2010, 40:1849-1859.

1092. Forray A, Mayes LC, Magriples U, Epperson CN: Prevalence of posttraumatic stress disorder in pregnant women with prior pregnancy complications. J Matern Fetal Neonatal Med 2009, 22:522-527.

1093. Banhidy F, Acs N, Puho E, Czeizel AE: Association between maternal panic disorders and pregnancy complications and delivery outcomes. Eur J Obstet Gynecol Reprod Biol 2006, 124:47-52.

1094. Lilliecreutz C, Sydsjo G, Josefsson A: Obstetric and perinatal outcomes among women with blood- and injection phobia during pregnancy. $J$ Affect Disord 2011, 129:289-295.
1095. Chen YH, Lin HC, Lee HC: Pregnancy outcomes among women with panic disorder - do panic attacks during pregnancy matter? J Affect Disord 2010, 120:258-262.

1096. Littleton HL, Breitkopf CR, Berenson AB: Correlates of anxiety symptoms during pregnancy and association with perinatal outcomes: a metaanalysis. Am J Obstet Gynecol 2007, 196:424-432.

1097. Rogal SS, Poschman K, Belanger K, Howell HB, Smith MV, Medina J, Yonkers KA: Effects of posttraumatic stress disorder on pregnancy outcomes. J Affect Disord 2007, 102:137-143.

1098. Newport DJ, Ji S, Long Q, Knight BT, Zach EB, Smith EN, Morris NJ, Stowe ZN: Maternal depression and anxiety differentially impact fetal exposures during pregnancy. J Clin Psychiatry 2012, 73:247-251.

1099. Lee AM, Lam SK, Sze Mun Lau SM, Chong CS, Chui HW, Fong DY: Prevalence, course, and risk factors for antenatal anxiety and depression. Obstet Gynecol 2007, 110:1102-1112.

1100. Challacombe F, Salkovskis P: A preliminary investigation of the impact of maternal obsessive-compulsive disorder and panic disorder on parenting and children. J Anxiety Disord 2009, 23:848-857.

1101. Zelkowitz P, Na S, Wang T, Bardin C, Papageorgiou A: Early maternal anxiety predicts cognitive and behavioural outcomes of VLBW children at 24 months corrected age. Acta Paediatr 2011, 100:700-704.

1102. O'Connor T. Heron J, Glover V: Antenatal anxiety predicts child behavioral/emotional problems independently of postnatal depression. J Am Acad Child Adolesc Psychiatry 2002, 41:1470-1477.

1103. Martini J, Knappe S, Beesdo-Baum K, Lieb R, Wittchen HU: Anxiety disorders before birth and self-perceived distress during pregnancy: associations with maternal depression and obstetric, neonatal and early childhood outcomes. Early Hum Dev 2010, 86:305-310.

1104. Seekles W, Cuijpers P, Kok R, Beekman A, van Marwijk H, van Straten A: Psychological treatment of anxiety in primary care: a meta-analysis. Psychol Med 2012, :1-11.

1105. Lilliecreutz C, Josefsson A, Sydsjo G: An open trial with cognitive behavioral therapy for blood- and injection phobia in pregnant women-a group intervention program. Arch Womens Ment Health 2010, 13:259-265

1106. Challacombe FL, Salkovskis PM: Intensive cognitive-behavioural treatment for women with postnatal obsessive-compulsive disorder: a consecutive case series. Behav Res Ther 2011, 49:422-426.

1107. Arch JJ, Dimidjian S, Chessick C: Are exposure-based cognitive behavioral therapies safe during pregnancy? Arch Womens Ment Health 2012, 15:445-457.

1108. ACOG Practice Bulletin: Clinical management guidelines for obstetriciangynecologists number 92, April 2008 (replaces practice bulletin number 87, November 2007). Use of psychiatric medications during pregnancy and lactation. Obstet Gynecol 2008, 111:1001-1020.

1109. Law R, Bozzo P, Koren G, Einarson A: FDA pregnancy risk categories and the CPS: do they help or are they a hindrance? Can Fam Physician 2010, 56:239-241

1110. Einarson A, Choi J, Einarson TR, Koren G: Incidence of major malformations in infants following antidepressant exposure in pregnancy: results of a large prospective cohort study. Can J Psychiatry 2009, 54:242-246.

1111. Malm H, Artama M, Gissler M, Ritvanen A: Selective serotonin reuptake inhibitors and risk for major congenital anomalies. Obstet Gynecol 2011, 118:111-120.

1112. Bellantuono C, Migliarese G, Gentile S: Serotonin reuptake inhibitors in pregnancy and the risk of major malformations: a systematic review. Hum Psychopharmacol 2007, 22:121-128.

1113. Grigoriadis S, Vonderporten EH, Mamisashvili L, Roerecke M, Rehm J, Dennis CL, Koren G, Steiner M, Mousmanis P, Cheung A, Ross LE: Antidepressant exposure during pregnancy and congenital malformations: is there an association? a systematic review and metaanalysis of the best evidence. J Clin Psychiatry 2013, 74:e293-308.

1114. Reis M, Kallen B: Delivery outcome after maternal use of antidepressant drugs in pregnancy: an update using Swedish data. Psychol Med 2010, 40:1723-1733.

1115. Bar-Oz B, Einarson T, Einarson A, Boskovic R, O'Brien L, Malm H, Berard A, Koren G: Paroxetine and congenital malformations: meta-analysis and consideration of potential confounding factors. Clin Ther 2007, 29:918-926. 
1116. Wurst KE, Poole C, Ephross SA, Olshan AF: First trimester paroxetine use and the prevalence of congenital, specifically cardiac, defects: a metaanalysis of epidemiological studies. Birth Defects Res A Clin Mol Teratol 2010, 88:159-170.

1117. Important safety information on Paxil (paroxetine) and increased risk of cardiac defects following exposure during first trimester of pregnancy. 2005 [http://www.healthycanadians.gc.ca/recall-alert-rappel-avis/hc-sc/ 2005/14173a-eng.php].

1118. Ross LE, Grigoriadis S, Mamisashvili L, Vonderporten EH, Roerecke M, Rehm J, Dennis CL, Koren G, Steiner M, Mousmanis P, Cheung A: Selected pregnancy and delivery outcomes after exposure to antidepressant medication: $\mathrm{A}$ systematic review and meta-analysis. JAMA Psychiatry 2013, 70:436-443.

1119. Hemels ME, Einarson A, Koren G, Lanctot KL, Einarson TR: Antidepressant use during pregnancy and the rates of spontaneous abortions: a metaanalysis. Ann Pharmacother 2005, 39:803-809.

1120. Rahimi R, Nikfar S, Abdollahi M: Pregnancy outcomes following exposure to serotonin reuptake inhibitors: a meta-analysis of clinical trials. Reprod Toxicol 2006, 22:571-575

1121. Grigoriadis S, Vonderporten EH, Mamisashvili L, Eady A, Tomlinson G, Dennis CL, Koren G, Steiner M, Mousmanis P, Cheung A, Ross LE: The effect of prenatal antidepressant exposure on neonatal adaptation: a systematic review and meta-analysis. J Clin Psychiatry 2013, 74:e309-320.

1122. Moses-Kolko EL, Bogen D, Perel J, Bregar A, Uhl K, Levin B, Wisner KL: Neonatal signs after late in utero exposure to serotonin reuptake inhibitors: literature review and implications for clinical applications. JAMA 2005, 293:2372-2383.

1123. Lattimore KA, Donn SM, Kaciroti N, Kemper AR, Neal CR Jr., Vazquez DM: Selective serotonin reuptake inhibitor (SSRI) use during pregnancy and effects on the fetus and newborn: a meta-analysis. J Perinatol 2005, 25:595-604

1124. Chambers CD, Hernandez-Diaz S, Van Marter LJ, Werler MM, Louik C, Jones KL, Mitchell AA: Selective serotonin-reuptake inhibitors and risk of persistent pulmonary hypertension of the newborn. N Engl J Med 2006, 354:579-587.

1125. Kieler H, Artama M, Engeland A, Ericsson O, Furu K, Gissler M, Nielsen RB, Norgaard M, Stephansson O, Valdimarsdottir U, et al: Selective serotonin reuptake inhibitors during pregnancy and risk of persistent pulmonary hypertension in the newborn: population based cohort study from the five Nordic countries. BMJ 2012, 344:d8012.

1126. Andrade SE, McPhillips H, Loren D, Raebel MA, Lane K, Livingston J, Boudreau DM, Smith DH, Davis RL, Willy ME, Platt R: Antidepressant medication use and risk of persistent pulmonary hypertension of the newborn. Pharmacoepidemiol Drug Saf 2009, 18:246-252.

1127. Wilson KL, Zelig CM, Harvey JP, Cunningham BS, Dolinsky BM, Napolitano PG: Persistent pulmonary hypertension of the newborn is associated with mode of delivery and not with maternal use of selective serotonin reuptake inhibitors. Am J Perinatol 2011, 28:19-24.

1128. Gentile S: Neurodevelopmental effects of prenatal exposure to psychotropic medications. Depress Anxiety 2010, 27:675-686.

1129. Gentile S, Galbally M: Prenatal exposure to antidepressant medications and neurodevelopmental outcomes: a systematic review. J Affect Disord 2011, 128:1-9.

1130. Udechuku A, Nguyen T, Hill R, Szego K: Antidepressants in pregnancy: a systematic review. Aust N Z J Psychiatry 2010, 44:978-996.

1131. Nulman I, Koren G, Rovet J, Barrera M, Pulver A, Streiner D, Feldman B: Neurodevelopment of children following prenatal exposure to venlafaxine, selective serotonin reuptake inhibitors, or untreated maternal depression. Am J Psychiatry 2012, 169:1165-1174.

1132. Croen LA, Grether JK, Yoshida CK, Odouli R, Hendrick V: Antidepressant use during pregnancy and childhood autism spectrum disorders. Arch Gen Psychiatry 2011, 68:1104-1112.

1133. Rai D, Lee BK, Dalman C, Golding J, Lewis G, Magnusson C: Parental depression, maternal antidepressant use during pregnancy, and risk of autism spectrum disorders: population based case-control study. BMJ 2013, 346:f2059.

1134. Figueroa R: Use of antidepressants during pregnancy and risk of attention-deficit/hyperactivity disorder in the offspring. J Dev Behav Pediatr 2010, 31:641-648.

1135. Froehlich TE, Anixt JS, Loe IM, Chirdkiatgumchai V, Kuan L, Gilman RC: Update on environmental risk factors for attention-deficit/hyperactivity disorder. Current psychiatry reports 2011, 13:333-344.
1136. Berle JO, Spigset O: Antidepressant use during breastfeeding. Curr Womens Health Rev 2011, 7:28-34.

1137. Enato E, Moretti M, Koren G: The fetal safety of benzodiazepines: an updated meta-analysis. J Obstet Gynaecol Can 2011, 33:46-48.

1138. Altshuler LL, Cohen L, Szuba MP, Burt VK, Gitlin M, Mintz J: Pharmacologic management of psychiatric illness during pregnancy: dilemmas and guidelines. Am J Psychiatry 1996, 153:592-606.

1139. Eros E, Czeizel AE, Rockenbauer M, Sorensen HT, Olsen J: A populationbased case-control teratologic study of nitrazepam, medazepam, tofisopam, alprazolum and clonazepam treatment during pregnancy. Eur J Obstet Gynecol Reprod Biol 2002, 101:147-154.

1140. Kelly LE, Poon S, Madadi P, Koren G: Neonatal benzodiazepines exposure during breastfeeding. J Pediatr 2012, 161:448-451.

1141. Gentile S: Antipsychotic therapy during early and late pregnancy. A systematic review. Schizophr Bull 2010, 36:518-544.

1142. Einarson A, Boskovic R: Use and safety of antipsychotic drugs during pregnancy. J Psychiatr Pract 2009, 15:183-192.

1143. Oyebode F, Rastogi A, Berrisford G, Coccia F: Psychotropics in pregnancy: safety and other considerations. Pharmacol Ther 2012, 135:71-77.

1144. Newham JJ, Thomas SH, MacRitchie K, McElhatton PR, McAllisterWilliams RH: Birth weight of infants after maternal exposure to typical and atypical antipsychotics: prospective comparison study. $\mathrm{Br} J$ Psychiatry 2008, 192:333-337.

1145. Diav-Citrin O, Shechtman S, Ornoy S, Arnon J, Schaefer C, Garbis H, Clementi M, Ornoy A: Safety of haloperidol and penfluridol in pregnancy: a multicenter, prospective, controlled study. J Clin Psychiatry 2005, 66:317-322.

1146. McKenna K, Koren G, Tetelbaum M, Wilton L, Shakir S, Diav-Citrin O, Levinson A, Zipursky RB, Einarson A: Pregnancy outcome of women using atypical antipsychotic drugs: a prospective comparative study. J Clin Psychiatry 2005, 66:444-449, quiz 546.

1147. Lin HC, Chen IJ, Chen YH, Lee HC, Wu FJ: Maternal schizophrenia and pregnancy outcome: does the use of antipsychotics make a difference? Schizophr Res 2010, 116:55-60.

1148. Hironaka M, Kotani T, Sumigama S, Tsuda H, Mano Y, Hayakawa H, Tanaka S, Ozaki N, Tamakoshi K, Kikkawa F: Maternal mental disorders and pregnancy outcomes: a clinical study in a Japanese population. $J$ Obstet Gynaecol Res 2011, 37:1283-1289.

1149. Wichman CL: Atypical antipsychotic use in pregnancy: a retrospective review. Arch Womens Ment Health 2009, 12:53-57.

1150. Galbally M, Snellen M, Lewis AJ: A review of the use of psychotropic medication in pregnancy. Curr Opin Obstet Gynecol 2011, 23:408-414.

1151. Antipsychotic drugs: class labeling change - treatment during pregnancy and potential risk to newborns. [http://www.fda.gov/Safety/ MedWatch/SafetyInformation/SafetyAlertsforHumanMedicalProducts/ ucm244175.htm].

1152. FDA drug safety communication: antipsychotic drug labels updated on use during pregnancy and risk of abnormal muscle movements and withdrawal symptoms in newborns. [http://www.fda.gov/Drugs/ DrugSafety/ucm243903.htm]

1153. Antipsychotic drugs: labelling update regarding the risk of abnormal muscle movements and withdrawal symptoms in newborns exposed during pregnancy. [http://www.healthycanadians.gc.ca/recall-alert-rappelavis/hc-sc/2011/13616a-eng.php].

1154. Gentile S: Infant safety with antipsychotic therapy in breast-feeding: a systematic review. J Clin Psychiatry 2008, 69:666-673.

1155. Kessler RC, Avenevoli S, Costello EJ, Georgiades K, Green JG, Gruber MJ, He JP, Koretz D, McLaughlin KA, Petukhova M, et al: Prevalence, persistence, and sociodemographic correlates of DSM-IV disorders in the National Comorbidity Survey Replication Adolescent Supplement. Arch Gen Psychiatry 2012, 69:372-380.

1156. Merikangas KR, He JP, Burstein M, Swanson SA, Avenevoli S, Cui L, Benjet C, Georgiades K, Swendsen J: Lifetime prevalence of mental disorders in U.S. adolescents: results from the National Comorbidity Survey Replication-Adolescent Supplement (NCS-A). J Am Acad Child Adolesc Psychiatry 2010, 49:980-989

1157. Meltzer H, Vostanis P, Dogra N, Doos L, Ford T, Goodman R: Children's specific fears. Child Care Health Dev 2009, 35:781-789.

1158. Heyman I, Fombonne E, Simmons H, Ford T, Meltzer H, Goodman R: Prevalence of obsessive-compulsive disorder in the British nationwide survey of child mental health. Int Rev Psychiatry 2003, 15:178-184. 
1159. Apter A, Fallon TJ Jr., King RA, Ratzoni G, Zohar AH, Binder M, Weizman A, Leckman JF, Pauls DL, Kron S, Cohen DJ: Obsessive-compulsive characteristics: from symptoms to syndrome. J Am Acad Child AdolesC Psychiatry 1996, 35:907-912.

1160. Flament MF, Whitaker A, Rapoport JL, Davies M, Berg CZ, Kalikow K, Sceery W, Shaffer D: Obsessive compulsive disorder in adolescence: an epidemiological study. J Am Acad Child Adolesc Psychiatry 1988, 27:764-771

1161. Albano AM, Kendall PC: Cognitive behavioural therapy for children and adolescents with anxiety disorders: Clinical research advances. Int Rev Psychiatry 2002, 14:129-134.

1162. Kendall P, Brady E, Verduin T: Comorbidity in childhood anxiety disorders and treatment outcome. J Am Acad Child Adolesc Psychiatry 2001, 40:787-794.

1163. Verduin $T$, Kendall P: Differential occurrence of comorbidity within childhood anxiety disorders. J Clin Child Adolesc Psychol 2003, 32:290-295.

1164. Bernstein GA, Bernat DH, Davis AA, Layne AE: Symptom presentation and classroom functioning in a nonclinical sample of children with social phobia. Depress Anxiety 2008, 25:752-760.

1165. Layne AE, Bernat DH, Victor AM, Bernstein GA: Generalized anxiety disorder in a nonclinical sample of children: symptom presentation and predictors of impairment. J Anxiety Disord 2009, 23:283-289.

1166. Frojd S, Ranta K, Kaltiala-Heino R, Marttunen M: Associations of social phobia and general anxiety with alcohol and drug use in a community sample of adolescents. Alcohol Alcohol 2011, 46:192-199.

1167. Low NC, Lee SS, Johnson JG, Williams JB, Harris ES: The association between anxiety and alcohol versus cannabis abuse disorders among adolescents in primary care settings. Fam Pract 2008, 25:321-327.

1168. Reed PL, Anthony JC, Breslau N: Incidence of drug problems in young adults exposed to trauma and posttraumatic stress disorder: do early life experiences and predispositions matter? Arch Gen Psychiatry 2007, 64:1435-1442

1169. Cornelius JR, Kirisci L, Reynolds M, Clark DB, Hayes J, Tarter R: PTSD contributes to teen and young adult cannabis use disorders. Addict Behav 2010, 35:91-94.

1170. Forbes EE, Bertocci MA, Gregory AM, Ryan ND, Axelson DA, Birmaher B, Dahl RE: Objective sleep in pediatric anxiety disorders and major depressive disorder. J Am Acad Child Adolesc Psychiatry 2008, 47:148-155.

1171. Alfano CA, Ginsburg GS, Kingery JN: Sleep-related problems among children and adolescents with anxiety disorders. J Am Acad Child Adolesc Psychiatry 2007, 46:224-232

1172. Alfano CA, Kim KL: Objective sleep patterns and severity of symptoms in pediatric obsessive compulsive disorder: a pilot investigation. $J$ Anxiety Disord 2011, 25:835-839.

1173. Storch EA, Murphy TK, Lack CW, Geffken GR, Jacob ML, Goodman WK: Sleep-related problems in pediatric obsessive-compulsive disorder. $J$ Anxiety Disord 2008, 22:877-885.

1174. Ginsburg GS, Riddle MA, Davies M: Somatic symptoms in children and adolescents with anxiety disorders. J Am Acad Child Adolesc Psychiatry 2006, 45:1179-1187.

1175. Boden JM, Fergusson DM, Horwood LJ: Anxiety disorders and suicidal behaviours in adolescence and young adulthood: findings from a longitudinal study. Psychol Med 2007, 37:431-440.

1176. Andres S, Boget T, Lazaro L, Penades R, Morer A, Salamero M, CastroFornieles J: Neuropsychological performance in children and adolescents with obsessive-compulsive disorder and influence of clinical variables. Biol Psychiatry 2007, 61:946-951.

1177. Jacob ML, Morelen D, Suveg C, Brown Jacobsen AM, Whiteside SP: Emotional, behavioral, and cognitive factors that differentiate obsessive-compulsive disorder and other anxiety disorders in youth Anxiety Stress Coping 2012, 25:229-237.

1178. Hughes AA, Lourea-Waddell B, Kendall PC: Somatic complaints in children with anxiety disorders and their unique prediction of poorer academic performance. Child Psychiatry Hum Dev 2008, 39:211-220.

1179. Grills-Taquechel AE, Fletcher JM, Vaughn SR, Denton CA, Taylor P: Anxiety and inattention as predictors of achievement in early elementary school children. Anxiety Stress Coping 2013, 26:391-410.

1180. Tillfors M, Persson S, Willen M, Burk WJ: Prospective links between social anxiety and adolescent peer relations. J Adolesc 2012, 35:1255-1263.

1181. Teubert D, Pinquart M: A meta-analytic review on the prevention of symptoms of anxiety in children and adolescents. J Anxiety Disord 2011, 25:1046-1059.
1182. Barrett PM, Farrell LJ, Ollendick TH, Dadds M: Long-term outcomes of an Australian universal prevention trial of anxiety and depression symptoms in children and youth: an evaluation of the friends program. J Clin Child Adolesc Psychol 2006, 35:403-411.

1183. Simon E, Bogels SM, Voncken JM: Efficacy of child-focused and parentfocused interventions in a child anxiety prevention study. J Clin Child Adolesc Psychol 2011, 40:204-219.

1184. Manassis K, Wilansky-Traynor P, Farzan N, Kleiman V, Parker K, Sanford M: The feelings club: randomized controlled evaluation of school-based CBT for anxious or depressive symptoms. Depress Anxiety 2010, 27:945-952.

1185. Stallard P, Velleman R, Salter E, Howse I, Yule W, Taylor G: A randomised controlled trial to determine the effectiveness of an early psychological intervention with children involved in road traffic accidents. J Child Psychol Psychiatry 2006, 47:127-134

1186. Nugent NR, Christopher NC, Crow JP, Browne L, Ostrowski S, Delahanty DL: The efficacy of early propranolol administration at reducing PTSD symptoms in pediatric injury patients: a pilot study. J Trauma Stress 2010, 23:282-287

1187. Weisz JR, Jensen-Doss A, Hawley KM: Evidence-based youth psychotherapies versus usual clinical care: a meta-analysis of direct comparisons. Am Psychol 2006, 61:671-689.

1188. Chu BC, Harrison TL: Disorder-specific effects of CBT for anxious and depressed youth: a meta-analysis of candidate mediators of change. Clin Child Fam Psychol Rev 2007, 10:352-372.

1189. In-Albon T, Schneider S: Psychotherapy of childhood anxiety disorders: a meta-analysis. Psychother Psychosom 2007, 76:15-24.

1190. Silverman WK, Pina AA, Viswesvaran C: Evidence-based psychosocial treatments for phobic and anxiety disorders in children and adolescents. J Clin Child Adolesc Psychol 2008, 37:105-130.

1191. Cartwright-Hatton S, Roberts C, Chitsabesan P, Fothergill C, Harrington R: Systematic review of the efficacy of cognitive behaviour therapies for childhood and adolescent anxiety disorders. Br J Clin Psychol 2004, 43:421-436

1192. Baer S, Garland E: Pilot study of community-based cognitive behavioral group therapy for adolescents with social phobia. J Am Acad Child Adolesc Psychiatry 2005, 44:258-264

1193. Beidel D, Turner S, Morris T: Behavioral treatment of childhood social phobia. J Consult Clin Psychol 2000, 68:1072-1080.

1194. Herbert JD, Gaudiano BA, Rheingold AA, Moitra E, Myers VH, Dalrymple KL, Brandsma LL: Cognitive behavior therapy for generalized social anxiety disorder in adolescents: a randomized controlled trial. J Anxiety Disord 2009, 23:167-177.

1195. Piet J, Hougaard E, Hecksher MS, Rosenberg NK: A randomized pilot study of mindfulness-based cognitive therapy and group cognitivebehavioral therapy for young adults with social phobia. Scand J Psychol 2010, 51:503-410.

1196. Melfsen S, Kuhnemund M, Schwieger J, Warnke A, Stadler C, Poustka F, Stangier U: Cognitive behavioral therapy of socially phobic children focusing on cognition: a randomised wait-list control study. Child Adolesc Psychiatry Ment Health 2011, 5:5.

1197. Segool NK, Carlson JS: Efficacy of cognitive-behavioral and pharmacological treatments for children with social anxiety. Depress Anxiety 2008, 25:620-631.

1198. Pincus DB, May JE, Whitton SW, Mattis SG, Barlow DH: Cognitivebehavioral treatment of panic disorder in adolescence. J Clin Child Adolesc Psychol 2010, 39:638-649.

1199. Franklin ME, Sapyta J, Freeman JB, Khanna M, Compton S, Almirall D, Moore P, Choate-Summers M, Garcia A, Edson AL, et al: Cognitive behavior therapy augmentation of pharmacotherapy in pediatric obsessive-compulsive disorder: the Pediatric OCD Treatment Study II (POTS II) randomized controlled trial. JAMA 2011, 306:1224-1232.

1200. Williams TI, Salkovskis PM, Forrester L, Turner S, White H, Allsopp MA: A randomised controlled trial of cognitive behavioural treatment for obsessive compulsive disorder in children and adolescents. Eur Child Adolesc Psychiatry 2010, 19:449-456

1201. Bolton D, Williams T, Perrin S, Atkinson L, Gallop C, Waite P, Salkovskis P. Randomized controlled trial of full and brief cognitive-behaviour therapy and wait-list for paediatric obsessive-compulsive disorder. J Child Psychol Psychiatry 2011, 52:1269-1278. 
1202. Turner C, Heyman I, Futh A, Lovell K: A pilot study of telephone cognitive-behavioural therapy for obsessive-compulsive disorder in young people. Behav Cogn Psychother 2009, 37:469-474.

1203. O'Kearney RT, Anstey KJ, von Sanden C: Behavioural and cognitive behavioural therapy for obsessive compulsive disorder in children and adolescents. Cochrane Database Syst Rev 2006, CD004856.

1204. Watson HJ, Rees CS: Meta-analysis of randomized, controlled treatment trials for pediatric obsessive-compulsive disorder. J Child Psychol Psychiatry 2008, 49:489-498.

1205. Smith P, Yule W, Perrin S, Tranah T, Dalgleish T, Clark DM: Cognitivebehavioral therapy for PTSD in children and adolescents: a preliminary randomized controlled trial. J Am Acad Child Adolesc Psychiatry 2007, 46:1051-1061

1206. Nixon RD, Sterk J, Pearce A: A randomized trial of cognitive behaviour therapy and cognitive therapy for children with posttraumatic stress disorder following single-incident trauma. J Abnorm Child Psychol 2012 40:327-337.

1207. Kowalik J, Weller J, Venter J, Drachman D: Cognitive behavioral therapy for the treatment of pediatric posttraumatic stress disorder: a review and meta-analysis. J Behav Ther Exp Psychiatry 2011, 42:405-413.

1208. King $N$, Tonge $B$, Mullen $P$, Myerson N, Heyne $D$, Rollings S, Martin R, Ollendick T: Treating sexually abused children with posttraumatic stress symptoms: a randomized clinical trial. J Am Acad Child Adolesc Psychiatry 2000, 39:1347-1355

1209. Cohen J, Mannarino A: A treatment outcome study for sexually abused preschool children: initial findings. J Am Acad Child Adolesc Psychiatry 1996, 35:42-50.

1210. Scheeringa MS, Weems CF, Cohen JA, Amaya-Jackson L, Guthrie D: Trauma-focused cognitive-behavioral therapy for posttraumatic stress disorder in three-through six year-old children: a randomized clinical trial. J Child Psychol Psychiatry 2011, 52:853-860.

1211. Deblinger E, Mannarino AP, Cohen JA, Steer RA: A follow-up study of a multisite, randomized, controlled trial for children with sexual abuse-related PTSD symptoms. J Am Acad Child Adolesc Psychiatry 2006, 45:1474-1484.

1212. King N, Tonge B, Heyne D, Pritchard M, Rollings S, Young D, Myerson N, Ollendick T: Cognitive-behavioral treatment of school-refusing children: a controlled evaluation. J Am Acad Child Adolesc Psychiatry 1998, 37:395-403.

1213. Last C, Hansen C, Franco N: Cognitive-behavioral treatment of school phobia. J Am Acad Child Adolesc Psychiatry 1998, 37:404-411.

1214. Pina AA, Zerr AA, Gonzales NA, Ortiz CD: Psychosocial interventions for school refusal behavior in children and adolescents. Child Dev Perspect 2009, 3:11-20

1215. Heyne D, King NJ, Tonge BJ, Rollings S, Young D, Pritchard M, Ollendick TH: Evaluation of child therapy and caregiver training in the treatment of school refusal. J Am Acad Child Adolesc Psychiatry 2002, 41:687-695

1216. Schneider S, Blatter-Meunier J, Herren C, Adornetto C, In-Albon T, Lavallee K: Disorder-specific cognitive-behavioral therapy for separation anxiety disorder in young children: a randomized waiting-list-controlled trial. Psychother Psychosom 2011, 80:206-215.

1217. Manassis K, Mendlowitz S, Scapillato D, Avery D, Fiksenbaum L, Freire M, Monga S, Owens M: Group and individual cognitive-behavioral therapy for childhood anxiety disorders: a randomized trial. J Am Acad Child Adolesc Psychiatry 2002, 41:1423-1430

1218. O'Leary EM, Barrett P. Fjermestad KW: Cognitive-behavioral family treatment for childhood obsessive-compulsive disorder: a 7-year followup study. J Anxiety Disord 2009, 23:973-978.

1219. Richardson T, Stallard P, Velleman S: Computerised cognitive behavioural therapy for the prevention and treatment of depression and anxiety in children and adolescents: a systematic review. Clin Child Fam Psychol Rev 2010, 13:275-290

1220. Storch EA, Caporino NE, Morgan JR, Lewin AB, Rojas A, Brauer L, Larson MJ, Murphy TK: Preliminary investigation of web-camera delivered cognitive-behavioral therapy for youth with obsessivecompulsive disorder. Psychiatry Res 2011, 189:407-412

1221. Kendall P: Treating anxiety disorders in children: results of a randomized clinical trial. J Consult Clin Psychol 1994, 62:100-110.

1222. Kendall P, Flannery-Schroeder E, Panichelli-Mindel S, Southam-Gerow M, Henin A, Warman M: Therapy for youths with anxiety disorders: a second randomized clinical trial. J Consult Clin Psychol 1997, 65:366-380.
1223. Walkup JT, Albano AM, Piacentini J, Birmaher B, Compton SN, Sherrill JT, Ginsburg GS, Rynn MA, McCracken J, Waslick B, et al: Cognitive behavioral therapy, sertraline, or a combination in childhood anxiety. N Engl I Med 2008, 359:2753-2766.

1224. Ginsburg GS, Kendall PC, Sakolsky D, Compton SN, Piacentini Albano AM, Walkup JT, Sherrill J, Coffey KA, Rynn MA, et al: Remission after acute treatment in children and adolescents with anxiety disorders: findings from the CAMS. J Consult Clin Psychol 2011, 79:806-813

1225. Kendall P, Safford S, Flannery-Schroeder E, Webb A: Child anxiety treatment: outcomes in adolescence and impact on substance use and depression at 7.4-year follow-up. J Consult Clin Psychol 2004, 72:276-287.

1226. Eldar S, Apter A, Lotan D, Edgar KP, Naim R, Fox NA, Pine DS, Bar-Haim Y: Attention bias modification treatment for pediatric anxiety disorders: a randomized controlled trial. Am J Psychiatry 2012, 169:213-230.

1227. Beidel DC, Turner SM, Sallee FR, Ammerman RT, Crosby LA, Pathak S: SET$C$ versus fluoxetine in the treatment of childhood social phobia. J Am Acad Child Adolesc Psychiatry 2007, 46:1622-1632.

1228. Beidel DC, Turner SM, Young BJ: Social effectiveness therapy for children: five years later. Behav Ther 2006, 37:416-425

1229. Simons M, Schneider S, Herpertz-Dahlmann B: Metacognitive therapy versus exposure and response prevention for pediatric obsessivecompulsive disorder. A case series with randomized allocation. Psychother Psychosom 2006, 75:257-264.

1230. Bolton D, Perrin S: Evaluation of exposure with response-prevention for obsessive compulsive disorder in childhood and adolescence. J Behav Ther Exp Psychiatry 2008, 39:11-22.

1231. Storch EA, Geffken GR, Merlo LJ, Mann G, Duke D, Munson M, Adkins J, Grabill KM, Murphy TK, Goodman WK: Family-based cognitive-behavioral therapy for pediatric obsessive-compulsive disorder: comparison of intensive and weekly approaches. J Am Acad Child Adolesc Psychiatry 2007, 46:469-478.

1232. Barrett $P$, Healy-Farrell $L$, March J: Cognitive-behavioral family treatment of childhood obsessive-compulsive disorder: a controlled trial. J Am Acad Child Adolesc Psychiatry 2004, 43:46-62.

1233. Van der Oord S, Lucassen S, Van Emmerik AA, Emmelkamp PM: Treatment of post-traumatic stress disorder in children using cognitive behavioural writing therapy. Clin Psychol Psychother 2010, 17:240-249.

1234. Lesmana CB, Suryani LK, Jensen GD, Tiliopoulos N: A spiritual-hypnosis assisted treatment of children with PTSD after the 2002 Bali terrorist attack. Am J Clin Hypn 2009, 52:23-34.

1235. Ford JD, Steinberg KL, Hawke J, Levine J, Zhang W: Randomized trial comparison of emotion regulation and relational psychotherapies for PTSD with girls involved in delinquency. J Clin Child Adolesc Psychol 2012, 41:27-37.

1236. Gilboa-Schechtman E, Foa EB, Shafran N, Aderka IM, Powers MB, Rachamim $L$, Rosenbach L, Yadin E, Apter A: Prolonged exposure versus dynamic therapy for adolescent PTSD: a pilot randomized controlled trial. J Am Acad Child Adolesc Psychiatry 2010, 49:1034-1042.

1237. Ahmad A, Sundelin-Wahlsten V: Applying EMDR on children with PTSD. Eur Child Adolesc Psychiatry 2008, 17:127-132.

1238. Ahmad A, Larsson B, Sundelin-Wahlsten V: EMDR treatment for children with PTSD: results of a randomized controlled trial. Nord I Psychiatry 2007, 61:349-354.

1239. Barrett P, Dadds M, Rapee R: Family treatment of childhood anxiety: a controlled trial. J Consult Clin Psychol 1996, 64:333-342.

1240. Mendlowitz S, Manassis K, Bradley S, Scapillato D, Miezitis S, Shaw B: Cognitive-behavioral group treatments in childhood anxiety disorders: the role of parental involvement. J Am Acad Child Adolesc Psychiatry 1999, 38:1223-1229.

1241. Shortt A, Barrett P, Fox T: Evaluating the FRIENDS program: a cognitivebehavioral group treatment for anxious children and their parents. Clin Child Psychol 2001, 30:525-535.

1242. Nauta M, Scholing A, Emmelkamp P, Minderaa R: Cognitive-behavioral therapy for children with anxiety disorders in a clinical setting: no additional effect of a cognitive parent training. J Am Acad Child Adolesc Psychiatry 2003, 42:1270-1278.

1243. Bogels SM, Siqueland L: Family cognitive behavioral therapy for children and adolescents with clinical anxiety disorders. J Am Acad Child Adolesc Psychiatry 2006, 45:134-141. 
1244. Wood JJ, Piacentini JC, Southam-Gerow M, Chu BC, Sigman M: Family cognitive behavioral therapy for child anxiety disorders. J Am Acad Child Adolesc Psychiatry 2006, 45:314-321.

1245. Piacentini J, Bergman RL, Chang S, Langley A, Peris T, Wood JJ, McCracken J: Controlled comparison of family cognitive behavioral therapy and psychoeducation/relaxation training for child obsessivecompulsive disorder. J Am Acad Child Adolesc Psychiatry 2011, 50:1149-1161

1246. Cobham VE, Dadds MR, Spence SH: The role of parental anxiety in the treatment of childhood anxiety. J Consult Clin Psychol 1998, 66:893-905.

1247. Eisen $A R$, Raleigh $H$, Neuhoff CC: The unique impact of parent training for separation anxiety disorder in children. Behav Ther 2008, 39:195-206.

1248. Thienemann M, Moore P, Tompkins K: A parent-only group intervention for children with anxiety disorders: pilot study. J Am Acad Child Adolesc Psychiatry 2006, 45:37-46.

1249. Storch EA, Merlo LJ, Larson MJ, Geffken GR, Lehmkuhl HD, Jacob ML, Murphy TK, Goodman WK: Impact of comorbidity on cognitivebehavioral therapy response in pediatric obsessive-compulsive disorder J Am Acad Child Adolesc Psychiatry 2008, 47:583-592.

1250. Jarrett MA, Ollendick TH: Treatment of comorbid attention-deficit/ hyperactivity disorder and anxiety in children: A multiple baseline design analysis. J Consult Clin Psychol 2012, 80:239-244.

1251. Levy K, Hunt C, Heriot S: Treating comorbid anxiety and aggression in children. J Am Acad Child Adolesc Psychiatry 2007, 46:1111-1118.

1252. Najavits LM, Gallop RJ, Weiss RD: Seeking safety therapy for adolescent girls with PTSD and substance use disorder: a randomized controlled trial. J Behav Health Serv Res 2006, 33:453-463.

1253. Dadds MR, Holland DE, Laurens KR, Mullins M, Barrett PM, Spence SH: Early intervention and prevention of anxiety disorders in children: results at 2-year follow-up. J Consult Clin Psychol 1999, 67:145-150.

1254. Barrett P, Duffy A, Dadds M, Rapee R: Cognitive-behavioral treatment of anxiety disorders in children: long-term (6-year) follow-up. J Consult Clin Psychol 2001, 69:135-141.

1255. Connolly SD, Bernstein GA: Practice parameter for the assessment and treatment of children and adolescents with anxiety disorders. J Am Acad Child Adolesc Psychiatry 2007, 46:267-283.

1256. Practice parameter on the use of psychotropic medication in children and adolescents. J Am Acad Child Adolesc Psychiatry 2009, 48:961-973.

1257. Practice parameter for the assessment and treatment of children and adolescents with obsessive-compulsive disorder. J Am Acad Child Adolesc Psychiatry 2012, 51:98-113.

1258. Cohen JA, Bukstein O, Walter H, Benson SR, Chrisman A, Farchione TR, Hamilton J, Keable H, Kinlan J, Schoettle U, et al: Practice parameter for the assessment and treatment of children and adolescents with posttraumatic stress disorder. J Am Acad Child Adolesc Psychiatry 2010 49:414-430

1259. Bernstein G, Borchardt C, Perwien A, Crosby R, Kushner M, Thuras P, Last C: Imipramine plus cognitive-behavioral therapy in the treatment of school refusal. J Am Acad Child Adolesc Psychiatry 2000, 39:276-283.

1260. Reinblatt SP, Riddle MA: The pharmacological management of childhood anxiety disorders: a review. Psychopharmacology 2007, 191:67-86.

1261. Ipser JC, Stein DJ, Hawkridge S, Hoppe L: Pharmacotherapy for anxiety disorders in children and adolescents. Cochrane Database Syst Rev 2009, CD005170.

1262. Uthman OA, Abdulmalik J: Comparative efficacy and acceptability of pharmacotherapeutic agents for anxiety disorders in children and adolescents: a mixed treatment comparison meta-analysis. Curr Med Res Opin 2010, 26:53-59.

1263. Geller D, Biederman J, Stewart S, Mullin B, Martin A, Spencer T, Faraone S: Which SSRI? A meta-analysis of pharmacotherapy trials in pediatric obsessive-compulsive disorder. Am J Psychiatry 2003, 160:1919-1928.

1264. Alaghband-Rad J, Hakimshooshtary M: A randomized controlled clinical trial of citalopram versus fluoxetine in children and adolescents with obsessive-compulsive disorder (OCD). Eur Child Adolesc Psychiatry 2009, 18:131-135

1265. Coskun M, Zoroglu S: Efficacy and safety of fluoxetine in preschool children with obsessive-compulsive disorder. J Child Adolesc Psychopharmacol 2009, 19:297-300.

1266. Ercan ES, Kandulu R, Akyol Ardic U: Preschool children with obsessivecompulsive disorder and fluoxetine treatment. Eur Child Adolesc Psychiatry 2012, 21:169-172.
1267. Geller D, Hoog S, Heiligenstein J, Ricardi R, Tamura R, Kluszynski S, Jacobson J: Fluoxetine treatment for obsessive-compulsive disorder in children and adolescents: a placebo-controlled clinical trial. J Am Acad Child Adolesc Psychiatry 2001, 40:773-779.

1268. Riddle M, Scahill L, King R, Hardin M, Anderson G, Ort S, Smith J, Leckman J, Cohen D: Double-blind, crossover trial of fluoxetine and placebo in children and adolescents with obsessive-compulsive disorder. J Am Acad Child Adolesc Psychiatry 1992, 31:1062-1069.

1269. Liebowitz MR, Turner SM, Piacentini J, Beidel DC, Clarvit SR, Davies SO, Graae F, Jaffer M, Lin SH, Sallee FR, et al: Fluoxetine in children and adolescents with OCD: a placebo-controlled trial. J Am Acad Child Adolesc Psychiatry 2002, 41:1431-1438.

1270. Mukaddes NM, Abali O, Kaynak N: Citalopram treatment of children and adolescents with obsessive-compulsive disorder: a preliminary report. Psychiatry Clin Neurosci 2003, 57:405-408.

1271. Riddle M, Reeve E, Yaryura-Tobias J, Yang H, Claghorn J, Gaffney G, Greist J, Holland D, McConville B, Pigott T, Walkup J: Fluvoxamine for children and adolescents with obsessive-compulsive disorder: a randomized, controlled, multicenter trial. J Am Acad Child AdolesC Psychiatry 2001, 40:222-229.

1272. Geller DA, Wagner KD, Emslie G, Murphy T, Carpenter DJ, Wetherhold E, Perera $P$, Machin A, Gardiner C: Paroxetine treatment in children and adolescents with obsessive-compulsive disorder: a randomized, multicenter, double-blind, placebo-controlled trial. J Am Acad Child Adolesc Psychiatry 2004, 43:1387-1396.

1273. March J, Biederman J, Wolkow R, Safferman A, Mardekian J, Cook E, Cutler N, Dominguez R, Ferguson J, Muller B, et al: Sertraline in children and adolescents with obsessive-compulsive disorder: a multicenter randomized controlled trial. JAMA 1998, 280:1752-1756.

1274. De Veaugh-Geiss J, Moroz G, Biederman J, Cantwell D, Fontaine R, Greist J, Reichler R, Katz R, Landau P: Clomipramine hydrochloride in childhood and adolescent obsessive-compulsive disorder-a multicenter trial. J Am Acad Child Adolesc Psychiatry 1992, 31:45-49.

1275. Leonard HL, Swedo SE, Rapoport JL, Koby EV, Lenane MC, Cheslow DL Hamburger SD: Treatment of obsessive-compulsive disorder with clomipramine and desipramine in children and adolescents. A doubleblind crossover comparison. Arch Gen Psychiatry 1989, 46:1088-1092.

1276. Flament MF, Rapoport JL, Berg CJ, Sceery W, Kilts C, Mellstrom B, Linnoila M: Clomipramine treatment of childhood obsessive-compulsive disorder. A double-blind controlled study. Arch Gen Psychiatry 1985, 42:977-983.

1277. Birmaher B, Axelson D, Monk K, Kalas C, Clark D, Ehmann M, Bridge J, Heo J, Brent D: Fluoxetine for the treatment of childhood anxiety disorders. J Am Acad Child Adolesc Psychiatry 2003, 42:415-423.

1278. Fluvoxamine for the treatment of anxiety disorders in children and adolescents. The Research Unit on Pediatric Psychopharmacology Anxiety Study Group. N Engl J Med 2001, 344:1279-1285.

1279. Wagner K, Berard R, Stein M, Wetherhold E, Carpenter D, Perera P, Gee M, Davy K, Machin A: A multicenter, randomized, double-blind, placebocontrolled trial of paroxetine in children and adolescents with social anxiety disorder. Arch Gen Psychiatry 2004, 61:1153-1162.

1280. Isolan L, Pheula G, Salum GA Jr., Oswald S, Rohde LA, Manfro GG: An open-label trial of escitalopram in children and adolescents with social anxiety disorder. J Child Adolesc Psychopharmacol 2007, 17:751-760.

1281. Compton S, Grant P, Chrisman A, Gammon P, Brown V, March J: Sertraline in children and adolescents with social anxiety disorder: an open trial. $J$ Am Acad Child Adolesc Psychiatry 2001, 40:564-571.

1282. March JS, Entusah AR, Rynn M, Albano AM, Tourian KA: A randomized controlled trial of venlafaxine ER versus placebo in pediatric social anxiety disorder. Biol Psychiatry 2007, 62:1149-1154.

1283. Mrakotsky C, Masek B, Biederman J, Raches D, Hsin O, Forbes P, de Moor C, DeMaso DR, Gonzalez-Heydrich J: Prospective open-label pilot trial of mirtazapine in children and adolescents with social phobia. $J$ Anxiety Disord 2008, 22:88-97.

1284. Rynn MA, Siqueland L, Rickels K: Placebo-controlled trial of sertraline in the treatment of children with generalized anxiety disorder. $\mathrm{Am} J$ Psychiatry 2001, 158:2008-2014.

1285. Lepola $\mathrm{U}$, Leinonen $\mathrm{E}$, Koponen $\mathrm{H}$ : Citalopram in the treatment of earlyonset panic disorder and school phobia. Pharmacopsychiatry 1996, 29:30-32. 
1286. Robb AS, Cueva JE, Sporn J, Yang R, Vanderburg DG: Sertraline treatment of children and adolescents with posttraumatic stress disorder: a double-blind, placebo-controlled trial. J Child Adolesc Psychopharmacol 2010, 20:463-471.

1287. Kutcher SP, MacKenzie S: Successful clonazepam treatment of adolescents with panic disorder. J Clin Psychopharmacol 1988, 8:299-301.

1288. Biederman J: Clonazepam in the treatment of prepubertal children with panic-like symptoms. J Clin Psychiatry 1987, 48(Suppl):38-42.

1289. Ballenger JC, Carek DJ, Steele JJ, Cornish-McTighe D: Three cases of panic disorder with agoraphobia in children. Am J Psychiatry 1989, 146:922-924.

1290. Simeon JG, Ferguson HB, Knott V, Roberts N, Gauthier B, Dubois C, Wiggins D: Clinical, cognitive, and neurophysiological effects of alprazolam in children and adolescents with overanxious and avoidant disorders. J Am Acad Child Adolesc Psychiatry 1992, 31:29-33.

1291. Bernstein GA, Garfinkel BD, Borchardt CM: Comparative studies of pharmacotherapy for school refusal. J Am Acad Child Adolesc Psychiatry 1990, 29:773-781.

1292. Graae F, Milner J, Rizzotto L, Klein RG: Clonazepam in childhood anxiety disorders. J Am Acad Child Adolesc Psychiatry 1994, 33:372-376.

1293. Masi G, Pfanner C, Millepiedi S, Berloffa S: Aripiprazole augmentation in 39 adolescents with medication-resistant obsessive-compulsive disorder. J Clin Psychopharmacol 2010, 30:688-693.

1294. Grant P, Lougee L, Hirschtritt M, Swedo SE: An open-label trial of riluzole, a glutamate antagonist, in children with treatment-resistant obsessivecompulsive disorder. J Child Adolesc Psychopharmacol 2007, 17:761-767.

1295. Storch EA, Murphy TK, Goodman WK, Geffken GR, Lewin AB, Henin A, Micco JA, Sprich S, Wilhelm S, Bengtson M, Geller DA: A preliminary study of $d$-cycloserine augmentation of cognitive-behavioral therapy in pediatric obsessive-compulsive disorder. Biol Psychiatry 2010, 68:1073-1076

1296. Larun L, Nordheim LV, Ekeland E, Hagen KB, Heian F: Exercise in prevention and treatment of anxiety and depression among children and young people. Cochrane Database Syst Rev 2006, 3:CD004691.

1297. Newman CL, Motta RW: The effects of aerobic exercise on childhood PTSD, anxiety, and depression. Int J Emerg Ment Health 2007, 9:133-158.

1298. Diaz AB, Motta R: The effects of an aerobic exercise program on posttraumatic stress disorder symptom severity in adolescents. Int $\mathrm{J}$ Emerg Ment Health 2008, 10:49-59.

1299. Harris E, Eng HY, Kowatch R, Delgado SV, Saldana SN: Disinhibition as a side effect of treatment with fluvoxamine in pediatric patients with obsessive-compulsive disorder. J Child Adolesc Psychopharmacol 2010, 20:347-353.

1300. Martin A, Young C, Leckman JF, Mukonoweshuro C, Rosenheck R, Leslie D: Age effects on antidepressant-induced manic conversion. Arch Pediatr Adolesc Med 2004, 158:773-780.

1301. Findling RL, Nucci G, Piergies AA, Gomeni R, Bartolic El, Fong R, Carpenter DJ, Leeder JS, Gaedigk A, Danoff TM: Multiple dose pharmacokinetics of paroxetine in children and adolescents with major depressive disorder or obsessive-compulsive disorder. Neuropsychopharmacology 2006, 31:1274-1285.

1302. Bridge JA, lyengar S, Salary CB, Barbe RP, Birmaher B, Pincus HA, Ren L, Brent DA: Clinical response and risk for reported suicidal ideation and suicide attempts in pediatric antidepressant treatment: a meta-analysis of randomized controlled trials. JAMA 2007, 297:1683-1696.

1303. Safer DJ, Zito JM: Treatment-emergent adverse events from selective serotonin reuptake inhibitors by age group: children versus adolescents. J Child Adolesc Psychopharmacol 2006, 16:159-169.

1304. Gum AM, King-Kallimanis B, Kohn R: Prevalence of mood, anxiety, and substance-abuse disorders for older Americans in the National Comorbidity Survey-Replication. Am J Geriatr Psychiatry 2009, 17:769-781.

1305. Grenier S, Preville M, Boyer R, O'Connor K, Beland SG, Potvin O, Hudon C, Brassard J: The impact of DSM-IV symptom and clinical significance criteria on the prevalence estimates of subthreshold and threshold anxiety in the older adult population. Am J Geriatr Psychiatry 2011, 19:316-326.

1306. Streiner DL, Cairney J, Veldhuizen S: The epidemiology of psychological problems in the elderly. Can J Psychiatry 2006, 51:185-191.

1307. Hollingworth SA, Burgess PM, Whiteford HA: Affective and anxiety disorders: prevalence, treatment and antidepressant medication use. Aust N Z J Psychiatry 2010, 44:513-519.
1308. Jorm A: Does old age reduce the risk of anxiety and depression? A review of epidemiological studies across the adult life span. Psychol Med 2000, 30:11-22.

1309. Calleo J, Stanley MA, Greisinger A, Wehmanen O, Johnson M, Novy D, Wilson N, Kunik M: Generalized anxiety disorder in older medical patients: diagnostic recognition, mental health management and service utilization. J Clin Psychol Med Settings 2009, 16:178-185.

1310. Cairney J, Corna LM, Streiner DL: Mental health care use in later life: results from a national survey of Canadians. Can J Psychiatry 2010, 55:157-164.

1311. Byers AL, Arean PA, Yaffe K: Low use of mental health services among older Americans with mood and anxiety disorders. Psychiatr Serv 2012, 63:66-72.

1312. Scott T, Mackenzie CS, Chipperfield JG, Sareen J: Mental health service use among Canadian older adults with anxiety disorders and clinically significant anxiety symptoms. Aging Ment Health 2010, 14:790-800.

1313. Brenes GA, Miller ME, Stanley MA, Williamson JD, Knudson M, McCall WV: Insomnia in older adults with generalized anxiety disorder. Am J Geriatr Psychiatry 2009, 17:465-472.

1314. Spira AP, Stone K, Beaudreau SA, Ancoli-Israel S, Yaffe K: Anxiety symptoms and objectively measured sleep quality in older women. Am J Geriatr Psychiatry 2009, 17:136-143.

1315. Mehta K, Simonsick E, Penninx B, Schulz R, Rubin S, Satterfield S, Yaffe K: Prevalence and correlates of anxiety symptoms in well-functioning older adults: findings from the health aging and body composition study. J Am Geriatr Soc 2003, 51:499-504.

1316. Beaudreau SA, O'Hara R: The association of anxiety and depressive symptoms with cognitive performance in community-dwelling older adults. Psychol Aging 2009, 24:507-512.

1317. Butters MA, Bhalla RK, Andreescu C, Wetherell JL, Mantella R, Begley AE, Lenze EJ: Changes in neuropsychological functioning following treatment for late-life generalised anxiety disorder. Br J Psychiatry 2011, 199:211-218.

1318. Sinoff G, Werner P: Anxiety disorder and accompanying subjective memory loss in the elderly as a predictor of future cognitive decline. Int J Geriatr Psychiatry 2003, 18:951-959.

1319. Scott Mackin R, Lesselyong JA, Yaffe K: Pattern of cognitive impairment in older veterans with posttraumatic stress disorder evaluated at a memory disorders clinic. Int J Geriatr Psychiatry 2012, 27:637-642.

1320. Mehta KM, Yaffe K, Brenes GA, Newman AB, Shorr RI, Simonsick EM, Ayonayon HN, Rubin SM, Covinsky KE: Anxiety symptoms and decline in physical function over 5 years in the health, aging and body composition study. J Am Geriatr Soc 2007, 55:265-270.

1321. Porensky EK, Dew MA, Karp JF, Skidmore E, Rollman BL, Shear MK, Lenze EJ: The burden of late-life generalized anxiety disorder: effects on disability, health-related quality of life, and healthcare utilization. Am J Geriatr Psychiatry 2009, 17:473-482.

1322. Diefenbach GJ, Tolin DF, Gilliam CM: Impairments in life quality among clients in geriatric home care: associations with depressive and anxiety symptoms. Int I Geriatr Psychiatry 2012, 27:828-835.

1323. Cairney J, Corna LM, Veldhuizen S, Herrmann N, Streiner DL: Comorbid depression and anxiety in later life: patterns of association, subjective well-being, and impairment. Am J Geriatr Psychiatry 2008, 16:201-208.

1324. King-Kallimanis B, Gum AM, Kohn R: Comorbidity of depressive and anxiety disorders for older Americans in the National Comorbidity Survey-Replication. Am J Geriatr Psychiatry 2009, 17:782-792.

1325. Wetherell JL, Ayers CR, Nuevo R, Stein MB, Ramsdell J, Patterson TL: Medical conditions and depressive, anxiety, and somatic symptoms in older adults with and without generalized anxiety disorder. Aging Ment Health 2010, 14:764-768.

1326. Schoevers R, Deeg D, van Tilburg W, Beekman A: Depression and generalized anxiety disorder: co-occurrence and longitudinal patterns in elderly patients. Am J Geriatr Psychiatry 2005, 13:31-39.

1327. Wolitzky-Taylor KB, Castriotta N, Lenze EJ, Stanley MA, Craske MG: Anxiety disorders in older adults: a comprehensive review. Depress Anxiety 2010, 27:190-211.

1328. Qureshi SU, Kimbrell T, Pyne JM, Magruder KM, Hudson TJ, Petersen NJ, Yu HJ, Schulz PE, Kunik ME: Greater prevalence and incidence of dementia in older veterans with posttraumatic stress disorder. $J \mathrm{Am}$ Geriatr Soc 2010, 58:1627-1633. 
1329. Vogele C, von Leupoldt A: Mental disorders in chronic obstructive pulmonary disease (COPD). Respir Med 2008, 102:764-773.

1330. Smoller JW, Pollack MH, Wassertheil-Smoller S, Jackson RD, Oberman A Wong ND, Sheps D: Panic attacks and risk of incident cardiovascular events among postmenopausal women in the Women's Health Initiative Observational Study. Arch Gen Psychiatry 2007, 64:1153-1160.

1331. Tully PJ, Baker RA, Knight JL: Anxiety and depression as risk factors for mortality after coronary artery bypass surgery. J Psychosom Res 2008, 64:285-290.

1332. Bryant $\mathrm{C}$, Jackson $H$, Ames D: The prevalence of anxiety in older adults: methodological issues and a review of the literature. J Affect Disord 2008, 109:233-250.

1333. Mohlman J, Bryant C, Lenze EJ, Stanley MA, Gum A, Flint A, Beekman AT, Wetherell JL, Thorp SR, Craske MG: Improving recognition of late life anxiety disorders in Diagnostic and Statistical Manual of Mental Disorders, Fifth Edition: observations and recommendations of the Advisory Committee to the Lifespan Disorders Work Group. Int I Geriatr Psychiatry 2012, 27:549-556.

1334. Brenes GA, Knudson M, McCall WV, Williamson JD, Miller ME, Stanley MA: Age and racial differences in the presentation and treatment of generalized anxiety disorder in primary care. J Anxiety Disord 2008, 22:1128-1136.

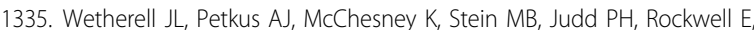
Sewell DD, Patterson TL: Older adults are less accurate than younger adults at identifying symptoms of anxiety and depression. J Nerv Ment Dis 2009, 197:623-626

1336. Jeste DV, Blazer DG, First M: Aging-related diagnostic variations: need for diagnostic criteria appropriate for elderly psychiatric patients. Biol Psychiatry 2005, 58:265-271.

1337. Ayers CR, Sorrell JT, Thorp SR, Wetherell JL: Evidence-based psychological treatments for late-life anxiety. Psychol Aging 2007, 22:8-17.

1338. Pinquart M, Duberstein PR: Treatment of anxiety disorders in older adults: a meta-analytic comparison of behavioral and pharmacological interventions. Am J Geriatr Psychiatry 2007, 15:639-651.

1339. Goncalves DC, Byrne GJ: Interventions for generalized anxiety disorder in older adults: systematic review and meta-analysis. J Anxiety Disord 2012, 26:1-11

1340. Hendriks GJ, Oude Voshaar RC, Keijsers GP, Hoogduin CA, van Balkom AJ: Cognitive-behavioural therapy for late-life anxiety disorders: a systematic review and meta-analysis. Acta Psychiatr Scand 2008 117:403-411.

1341. Thorp SR, Ayers CR, Nuevo R, Stoddard JA, Sorrell JT, Wetherell JL: Metaanalysis comparing different behavioral treatments for late-life anxiety. Am J Geriatr Psychiatry 2009, 17:105-115.

1342. Gould RL, Coulson MC, Howard RJ: Efficacy of cognitive behavioral therapy for anxiety disorders in older people: a meta-analysis and meta-regression of randomized controlled trials. J Am Geriatr Soc 2012, 60:218-229.

1343. Mohlman J, Gorenstein E, Kleber M, de JM, Gorman J, Papp L: Standard and enhanced cognitive-behavior therapy for late-life generalized anxiety disorder: two pilot investigations. Am J Geriatr Psychiatry 2003, 11:24-32.

1344. Stanley M, Hopko D, Diefenbach G, Bourland S, Rodriguez H, Wagener P: Cognitive-behavior therapy for late-life generalized anxiety disorder in primary care: preliminary findings. Am J Geriatr Psychiatry 2003, 11:92-96

1345. Bradford A, Cully J, Rhoades H, Kunik M, Kraus-Schuman C, Wilson N, Stanley M: Early response to psychotherapy and long-term change in worry symptoms in older adults with generalized anxiety disorder. Am J Geriatr Psychiatry 2011, 19:347-356.

1346. Stanley MA, Wilson NL, Novy DM, Rhoades HM, Wagener PD, Greisinger AJ, Cully JA, Kunik ME: Cognitive behavior therapy for generalized anxiety disorder among older adults in primary care: a randomized clinical trial. JAMA 2009, 301:1460-1467.

1347. Wetherell J, Gatz M, Craske M: Treatment of generalized anxiety disorder in older adults. J Consult Clin Psychol 2003, 71:31-40.

1348. Hendriks GJ, Keijsers GP, Kampman M, Oude Voshaar RC, Verbraak MJ, Broekman TG, Hoogduin CA: A randomized controlled study of paroxetine and cognitive-behavioural therapy for late-life panic disorder. Acta Psychiatr Scand 2010, 122:11-19.

1349. Schuurmans J, Comijs H, Emmelkamp PM, Gundy CM, Weijnen I, van den Hout $M$, van Dyck R: A randomized, controlled trial of the effectiveness of cognitive-behavioral therapy and sertraline versus a waitlist control group for anxiety disorders in older adults. Am J Geriatr Psychiatry 2006, $14: 255-263$

1350. Thorp SR, Stein MB, Jeste DV, Patterson TL, Wetherell JL: Prolonged exposure therapy for older veterans with posttraumatic stress disorder: a pilot study. Am J Geriatr Psychiatry 2012, 20:276-280.

1351. Pachana NA, Woodward RM, Byrne GJ: Treatment of specific phobia in older adults. Clin Interv Aging 2007, 2:469-476.

1352. Brenes GA, Miller ME, Williamson JD, McCall WV, Knudson M, Stanley MA: A randomized controlled trial of telephone-delivered cognitivebehavioral therapy for late-life anxiety disorders. Am J Geriatr Psychiatry 2012, 20:707-716

1353. Pasco JA, Williams LJ, Jacka FN, Henry MJ, Coulson CE, Brennan SL, Leslie E, Nicholson GC, Kotowicz MA, Berk M: Habitual physical activity and the risk for depressive and anxiety disorders among older men and women. Int Psychogeriatr 2011, 23:292-298.

1354. Montgomery S, Chatamra K, Pauer L, Whalen E, Baldinetti F: Efficacy and safety of pregabalin in elderly people with generalised anxiety disorder. Br J Psychiatry 2008, 193:389-394.

1355. Karaiskos D, Pappa D, Tzavellas E, Siarkos K, Katirtzoglou E, Papadimitriou GN, Politis A: Pregabalin augmentation of antidepressants in older patients with comorbid depression and generalized anxiety disorder-an open-label study. Int J Geriatr Psychiatry 2013, 28:100-105.

1356. Davidson J, Allgulander C, Pollack MH, Hartford J, Erickson JS, Russell JM, Perahia D, Wohlreich MM, Carlson J, Raskin J: Efficacy and tolerability of duloxetine in elderly patients with generalized anxiety disorder: a pooled analysis of four randomized, double-blind, placebo-controlled studies. Hum Psychopharmacol 2008, 23:519-526.

1357. Lenze EJ, Mulsant BH, Shear MK, Dew MA, Miller MD, Pollock BG, Houck P, Tracey B, Reynolds CF 3rd: Efficacy and tolerability of citalopram in the treatment of late-life anxiety disorders: results from an 8-week randomized, placebo-controlled trial. Am J Psychiatry 2005, 162:146-150.

1358. Blank S, Lenze EJ, Mulsant BH, Dew MA, Karp JF, Shear MK, Houck PR, Miller MD, Pollock BG, Tracey B, Reynolds CF: Outcomes of late-life anxiety disorders during 32 weeks of citalopram treatment. J Clin Psychiatry 2006, 67:468-472.

1359. Mohamed S, Osatuke K, Aslam M, Kasckow J: Escitalopram for comorbid depression and anxiety in elderly patients: a 12-week, open-label, flexible-dose, pilot trial. Am J Geriatr Pharmacother 2006, 4:201-209.

1360. Schuurmans J, Comijs H, Emmelkamp PM, Weijnen IJ, van den Hout M, van Dyck R: Long-term effectiveness and prediction of treatment outcome in cognitive behavioral therapy and sertraline for late-life anxiety disorders. Int Psychogeriatr 2009, 21:1148-1159.

1361. Rampello L, Alvano A, Raffaele R, Malaguarnera M, Vecchio I: New possibilities of treatment for panic attacks in elderly patients: escitalopram versus citalopram. J Clin Psychopharmacol 2006, 26:67-70.

1362. Wylie M, Miller M, Shear M, Little J, Mulsant B, Pollock B, Reynolds C: Fluvoxamine pharmacotherapy of anxiety disorders in later life: preliminary open-trial data. J Geriatr Psychiatry Neurol 2000, 13:43-48.

1363. Gardner M, Malone D, Sey M, Babington M: Mirtazapine is associated with less anxiolytic use among elderly depressed patients in long-term care facilities. J Am Med Dir Assoc 2004, 5:101-106.

1364. Schatzberg A, Kremer C, Rodrigues H, Murphy G: Double-blind, randomized comparison of mirtazapine and paroxetine in elderly depressed patients. Am J Geriatr Psychiatry 2002, 10:541-550

1365. Benitez Cl, Smith K, Vasile RG, Rende R, Edelen MO, Keller MB: Use of benzodiazepines and selective serotonin reuptake inhibitors in middleaged and older adults with anxiety disorders: a longitudinal and prospective study. Am J Geriatr Psychiatry 2008, 16:5-13.

1366. Uchida H, Suzuki T, Mamo DC, Mulsant BH, Kikuchi T, Takeuchi H, Tomita M, Watanabe K, Yagi G, Kashima H: Benzodiazepine and antidepressant use in elderly patients with anxiety disorders: a survey of 796 outpatients in Japan. J Anxiety Disord 2009, 23:477-481.

1367. Preville M, Vasiliadis HM, Bosse C, Dionne PA, Voyer P, Brassard J: Pattern of psychotropic drug use among older adults having a depression or an anxiety disorder: results from the longitudinal ESA study. Can $J$ Psychiatry 2011, 56:348-357.

1368. Mallet $L$, Spinewine A, Huang A: The challenge of managing drug interactions in elderly people. Lancet 2007, 370:185-191.

1369. Hines LE, Murphy JE: Potentially harmful drug-drug interactions in the elderly: a review. Am J Geriatr Pharmacother 2011, 9:364-377. 
1370. Klotz U: Pharmacokinetics and drug metabolism in the elderly. Drug Metab Rev 2009, 41:67-76.

1371. Boyce RD, Handler SM, Karp JF, Hanlon JT: Age-related changes in antidepressant pharmacokinetics and potential drug-drug interactions: a comparison of evidence-based literature and package insert information. Am J Geriatr Pharmacother 2012, 10:139-150.

1372. Qato DM, Alexander GC, Conti RM, Johnson M, Schumm P, Lindau ST: Use of prescription and over-the-counter medications and dietary supplements among older adults in the United States. JAMA 2008, 300:2867-2878

1373. Takkouche B, Montes-Martinez A, Gill SS, Etminan M: Psychotropic medications and the risk of fracture: a meta-analysis. Drug Saf 2007, 30:171-184.

1374. Zint K, Haefeli WE, Glynn RJ, Mogun H, Avorn J, Sturmer T: Impact of drug interactions, dosage, and duration of therapy on the risk of hip fracture associated with benzodiazepine use in older adults. Pharmacoepidemiol Drug Saf 2010, 19:1248-1255.

1375. Ziere G, Dieleman JP, van der Cammen TJ, Hofman A, Pols HA, Stricker BH: Selective serotonin reuptake inhibiting antidepressants are associated with an increased risk of nonvertebral fractures. J Clin Psychopharmacol 2008, 28:411-417.

1376. Chatterjee S, Chen H, Johnson ML, Aparasu RR: Risk of falls and fractures in older adults using atypical antipsychotic agents: a propensity scoreadjusted, retrospective cohort study. Am J Geriatr Pharmacother 2012, 10:83-94.

1377. FDA requests boxed warnings on older class of antipsychotic drugs. [http://www.fda.gov/NewsEvents/Newsroom/PressAnnouncements/2008/ ucm116912.htm].

1378. Gill SS, Bronskill SE, Normand SL, Anderson GM, Sykora K, Lam K, Bell CM, Lee PE, Fischer HD, Herrmann N, et al: Antipsychotic drug use and mortality in older adults with dementia. Ann Intern Med 2007, 146:775-786.

1379. Kales HC, Kim HM, Zivin K, Valenstein M, Seyfried LS, Chiang C, Cunningham F, Schneider LS, Blow FC: Risk of mortality among individual antipsychotics in patients with dementia. Am J Psychiatry 2012, 169:71-79.

1380. Schneeweiss S, Setoguchi S, Brookhart A, Dormuth C, Wang PS: Risk of death associated with the use of conventional versus atypical antipsychotic drugs among elderly patients. CMAJ 2007, 176:627-632.

1381. Pizzi C, Rutjes AW, Costa GM, Fontana F, Mezzetti A, Manzoli L: Metaanalysis of selective serotonin reuptake inhibitors in patients with depression and coronary heart disease. Am J Cardiol 2011, 107:972-979.

1382. Meng X, D'Arcy C: Common and unique risk factors and comorbidity for 12-month mood and anxiety disorders among Canadians. Can J Psychiatry 2012, 57:479-487.

1383. Goldstein Bl, Levitt AJ: The specific burden of comorbid anxiety disorders and of substance use disorders in bipolar I disorder. Bipolar Disord 2008, 10:67-78.

1384. O'Neil KA, Podell JL, Benjamin CL, Kendall PC: Comorbid depressive disorders in anxiety-disordered youth: demographic, clinical, and family characteristics. Child Psychiatry Hum Dev 2010, 41:330-341.

1385. Green BL, Krupnick JL, Chung J, Siddique J, Krause ED, Revicki D, Frank L, Miranda J: Impact of PTSD comorbidity on one-year outcomes in a depression trial. J Clin Psychol 2006, 62:815-835.

1386. Andreescu C, Lenze EJ, Dew MA, Begley AE, Mulsant BH, Dombrovski AY, Pollock BG, Stack J, Miller MD, Reynolds CF: Effect of comorbid anxiety on treatment response and relapse risk in late-life depression: controlled study. Br J Psychiatry 2007, 190:344-349.

1387. Goes FS, McCusker MG, Bienvenu OJ, Mackinnon DF, Mondimore FM, Schweizer B, Depaulo JR, Potash JB: Co-morbid anxiety disorders in bipolar disorder and major depression: familial aggregation and clinical characteristics of co-morbid panic disorder, social phobia, specific phobia and obsessive-compulsive disorder. Psychol Med 2012. 42:1449-1459.

1388. Otto MW, Simon NM, Wisniewski SR, Miklowitz DJ, Kogan JN, ReillyHarrington NA, Frank E, Nierenberg AA, Marangell LB, Sagduyu K, et al: Prospective 12-month course of bipolar disorder in out-patients with and without comorbid anxiety disorders. Br J Psychiatry 2006, 189:20-25.

1389. Sala R, Goldstein BI, Morcillo C, Liu SM, Castellanos M, Blanco C: Course of comorbid anxiety disorders among adults with bipolar disorder in the U.S. population. J Psychiatr Res 2012, 46:865-872.
1390. McWilliams LA, Cox BJ, Enns MW: Mood and anxiety disorders associated with chronic pain: an examination in a nationally representative sample. Pain 2003, 106:127-133.

1391. Mittal D, Fortney JC, Pyne JM, Edlund MJ, Wetherell JL: Impact of comorbid anxiety disorders on health-related quality of life among patients with major depressive disorder. Psychiatr Serv 2006, 57:1731-1737.

1392. Katz C, Yaseen ZS, Mojtabai R, Cohen L, Galynker II: Panic as an independent risk factor for suicide attempt in depressive illness: findings from the National Epidemiological Survey on Alcohol and Related Conditions (NESARC). J Clin Psychiatry 2011, 72:1628-1635.

1393. Brown LA, Gaudiano BA, Miller IW: The impact of panic-agoraphobic comorbidity on suicidality in hospitalized patients with major depression. Depress Anxiety 2010, 27:310-315.

1394. Schaffer A, Mclntosh D, Goldstein BI, Rector NA, Mclntyre RS, Beaulieu S, Swinson R, Yatham LN: The CANMAT task force recommendations for the management of patients with mood disorders and comorbid anxiety disorders. Ann Clin Psychiatry 2012, 24:6-22.

1395. Silverstone P, Salinas E: Efficacy of venlafaxine extended release in patients with major depressive disorder and comorbid generalized anxiety disorder. J Clin Psychiatry 2001, 62:523-529.

1396. Maneeton N, Maneeton B, Srisurapanont M, Martin SD: Quetiapine monotherapy in acute phase for major depressive disorder: a metaanalysis of randomized, placebo-controlled trials. BMC Psychiatry 2012, $12: 160$.

1397. Maher AR, Maglione M, Bagley S, Suttorp M, Hu JH, Ewing B, Wang Z, Timmer M, Sultzer D, Shekelle PG: Efficacy and comparative effectiveness of atypical antipsychotic medications for off-label uses in adults: a systematic review and meta-analysis. JAMA 2011, 306:1359-1369.

1398. McIntyre A, Gendron A: Quetiapine adjunct to selective serotonin reuptake inhibitors or venlafaxine in patients with major depression, comorbid anxiety, and residual depressive symptoms: a randomized, placebo-controlled pilot study. Depress Anxiety 2007, 24:487-494.

1399. Merikangas KR, Akiskal HS, Angst J, Greenberg PE, Hirschfeld RM, Petukhova M, Kessler RC: Lifetime and 12-month prevalence of bipolar spectrum disorder in the National Comorbidity Survey replication. Arch Gen Psychiatry 2007, 64:543-552.

1400. Young S, Pfaff D, Lewandowski KE, Ravichandran C, Cohen BM, Ongur D: Anxiety disorder comorbidity in bipolar disorder, schizophrenia and schizoaffective disorder. Psychopathology 2013, 46:176-185.

1401. Achim AM, Maziade M, Raymond E, Olivier D, Merette C, Roy MA: How prevalent are anxiety disorders in schizophrenia? A meta-analysis and critical review on a significant association. Schizophr Bull 2011, 37:811-821

1402. Simon NM, Zalta AK, Otto MW, Ostacher MJ, Fischmann D, Chow CW, Thompson EH, Stevens JC, Demopulos CM, Nierenberg AA, Pollack MH: The association of comorbid anxiety disorders with suicide attempts and suicidal ideation in outpatients with bipolar disorder. $J$ Psychiatr Res 2007, 41:255-264

1403. Nakagawa A, Grunebaum MF, Sullivan GM, Currier D, Ellis SP, Burke AK, Brent DA, Mann JJ, Oquendo MA: Comorbid anxiety in bipolar disorder: does it have an independent effect on suicidality? Bipolar Disord 2008, 10:530-538.

1404. Pallanti S, Quercioli L, Hollander E: Social anxiety in outpatients with schizophrenia: a relevant cause of disability. Am J Psychiatry 2004, 161:53-58.

1405. Makara-Studzinska M, Wolyniak M, Krys K: Influence of anxiety and depression on quality of life of people with schizophrenia in the eastern region of poland. ISRN Psychiatry 2012, 2012:839324.

1406. Barnes TR: Evidence-based guidelines for the pharmacological treatment of schizophrenia: recommendations from the British Association for Psychopharmacology. J Psychopharmacol 2011, 25:567-620.

1407. Sheehan DV, McElroy SL, Harnett-Sheehan K, Keck PE Jr., Janavs J, Rogers J, Gonzalez R, Shivakumar G, Suppes T: Randomized, placebo-controlled trial of risperidone for acute treatment of bipolar anxiety. J Affect Disord 2009, 115:376-385.

1408. Maina G, Albert U, Rosso G, Bogetto F: Olanzapine or lamotrigine addition to lithium in remitted bipolar disorder patients with anxiety disorder comorbidity: a randomized, single-blind, pilot study. I Clin Psychiatry 2008, 69:609-616. 
1409. Hirschfeld RM, Weisler RH, Raines SR, Macfadden W: Quetiapine in the treatment of anxiety in patients with bipolar I or II depression: a secondary analysis from a randomized, double-blind, placebocontrolled study. J Clin Psychiatry 2006, 67:355-362.

1410. McElroy SL, Weisler RH, Chang W, Olausson B, Paulsson B, Brecher M, Agambaram V, Merideth C, Nordenhem A, Young AH: A double-blind, placebo-controlled study of quetiapine and paroxetine as monotherapy in adults with bipolar depression (EMBOLDEN II). J Clin Psychiatry 2010, 71:163-174.

1411. Young AH, McElroy SL, Bauer M, Philips N, Chang W, Olausson B, Paulsson B, Brecher M: A double-blind, placebo-controlled study of quetiapine and lithium monotherapy in adults in the acute phase of bipolar depression (EMBOLDEN I). J Clin Psychiatry 2010, 71:150-162

1412. Sagman D, Lee B, Chandresena R, Jones B, Brunner E: A Canadian naturalistic study of a community-based cohort treated for bipolar disorder. BMC Psychiatry 2010, 10:24.

1413. Tohen M, Calabrese J, Vieta E, Bowden C, Gonzalez-Pinto A, Lin D, Xu W, Corya S: Effect of comorbid anxiety on treatment response in bipolar depression. J Affect Disord 2007, 104:137-146.

1414. Perugi G, Toni C, Frare F, Ruffolo G, Moretti L, Torti C, Akiskal HS: Effectiveness of adjunctive gabapentin in resistant bipolar disorder: is it due to anxious-alcohol abuse comorbidity? J Clin Psychopharmacol 2002, 22:584-591.

1415. Davis LL, Bartolucci A, Petty F: Divalproex in the treatment of bipolar depression: a placebo-controlled study. J Affect Disord 2005, 85:259-266.

1416. Vieta E, Martinez-Aran A, Nieto E, Colom F, Reinares M, Benabarre A, Gasto C: Adjunctive gabapentin treatment of bipolar disorder. Eur Psychiatry 2000, 15:433-437.

1417. Wender PH, Wolf LE, Wasserstein J: Adults with ADHD. An overview. Ann N Y Acad Sci 2001, 931:1-16.

1418. Dulcan M: Practice parameters for the assessment and treatment of children, adolescents, and adults with attention-deficit/hyperactivity disorder. American Academy of Child and Adolescent Psychiatry. J Am Acad Child Adolesc Psychiatry 1997, 36:85S-121S

1419. Mannuzza S, Klein RG, Bessler A, Malloy P, LaPadula M: Adult outcome of hyperactive boys. Educational achievement, occupational rank, and psychiatric status. Arch Gen Psychiatry 1993, 50:565-576.

1420. Weiss G, Hechtman L: Hyperactive children grown up: ADHD in children, adolescents, and adults. New York, NY: Guilford Press; 1993.

1421. Pary R, Lewis S, Matuschka PR, Rudzinskiy P, Safi M, Lippmann S: Attention deficit disorder in adults. Ann Clin Psychiatry 2002, 14:105-111.

1422. Wilens TE, Biederman J, Spencer TJ: Attention deficit/hyperactivity disorder across the lifespan. Annu Rev Med 2002, 53:113-131.

1423. Wilens TE, Faraone SV, Biederman J: Attention-deficit/hyperactivity disorder in adults. JAMA 2004, 292:619-623.

1424. Garcia SP, Guimaraes J, Zampieri JF, Martinez AL, Polanczyk G, Rohde LA: Response to methylphenidate in children and adolescents with ADHD: does comorbid anxiety disorders matters? J Neural Transm 2009, 116:631-636.

1425. Blouin B, Maddeaux C, Stanley Firestone J, van Stralen J: Predicting response of $A D H D$ symptoms to methylphenidate treatment based on comorbid anxiety. J Atten Disord 2010, 13:414-419.

1426. Kratochvil CJ, Newcorn JH, Arnold LE, Duesenberg D, Emslie GJ, Quintana H, Sarkis EH, Wagner KD, Gao H, Michelson D, Biederman J: Atomoxetine alone or combined with fluoxetine for treating ADHD with comorbid depressive or anxiety symptoms. J Am Acad Child Adolesc Psychiatry 2005, 44:915-924.

1427. Goodman DW, Thase ME: Recognizing ADHD in adults with comorbid mood disorders: implications for identification and management. Postgrad Med 2009, 121:20-30

1428. Gabriel A, Violato C: Adjunctive atomoxetine to SSRIs or SNRIs in the treatment of adult ADHD patients with comorbid partially responsive generalized anxiety (GA): an open-label study. Atten Defic Hyperact Disord 2011, 3:319-326.

1429. Gabriel A: The mixed amphetamine salt extended release (Adderall XR Max-XR) as an adjunctive to SSRIS or SNRIS in the treatment of adult ADHD patients with comorbid partially responsive generalized anxiety: an open-label study. Atten Defic Hyperact Disord 2010, 2:87-92

1430. El-Gabalawy R, Mackenzie CS, Shooshtari S, Sareen J: Comorbid physical health conditions and anxiety disorders: a population-based exploration of prevalence and health outcomes among older adults. Gen Hosp Psychiatry 2011, 33:556-564.

1431. Vogelzangs $N$, Seldenrijk A, Beekman AT, van Hout HP, de Jonge $P$, Penninx BW: Cardiovascular disease in persons with depressive and anxiety disorders. J Affect Disord 2010, 125:241-248.

1432. Huang KL, Su TP, Chen TJ, Chou YH, Bai YM: Comorbidity of cardiovascular diseases with mood and anxiety disorder: a population based 4-year study. Psychiatry Clin Neurosci 2009, 63:401-409.

1433. Demyttenaere K, Bonnewyn A, Bruffaerts R, De Graaf R, Haro JM, Alonso J: Comorbid painful physical symptoms and anxiety: prevalence, work loss and help-seeking. J Affect Disord 2008, 109:264-272.

1434. Asmundson GJ, Katz J: Understanding the co-occurrence of anxiety disorders and chronic pain: state-of-the-art. Depress Anxiety 2009, 26:888-901

1435. Hartford JT, Endicott J, Kornstein SG, Allgulander C, Wohlreich MM, Russell JM, Perahia DG, Erickson JS: Implications of pain in generalized anxiety disorder: efficacy of duloxetine. Prim Care Companion J Clin Psychiatry 2008, 10:197-204.

1436. Beesdo K, Hartford J, Russell J, Spann M, Ball S, Wittchen HU: The shortand long-term effect of duloxetine on painful physical symptoms in patients with generalized anxiety disorder: results from three clinical trials. J Anxiety Disord 2009, 23:1064-1071.

1437. Russell JM, Weisberg R, Fava M, Hartford JT, Erickson JS, D'Souza DN: Efficacy of duloxetine in the treatment of generalized anxiety disorder in patients with clinically significant pain symptoms. Depress Anxiety 2008, 25:E1-11.

1438. Jackson JL, Shimeall W, Sessums L, Dezee KJ, Becher D, Diemer M, Berbano E, O'Malley PG: Tricyclic antidepressants and headaches: systematic review and meta-analysis. BMJ 2010, 341:c5222.

1439. Saarto T, Wiffen PJ: Antidepressants for neuropathic pain. Cochrane Database Syst Rev 2007, CD005454.

1440. Denollet J, Maas K, Knottnerus A, Keyzer JJ, Pop VJ: Anxiety predicted premature all-cause and cardiovascular death in a 10-year follow-up of middle-aged women. J Clin Epidemiol 2009, 62:452-456.

1441. Chamberlain AM, Vickers KS, Colligan RC, Weston SA, Rummans TA, Roger VL: Associations of preexisting depression and anxiety with hospitalization in patients with cardiovascular disease. Mayo Clin Proc 2011, 86:1056-1062.

1442. Doering LV, Moser DK, Riegel B, McKinley S, Davidson P, Baker $H_{t}$ Meischke H, Dracup K: Persistent comorbid symptoms of depression and anxiety predict mortality in heart disease. Int J Cardiol 2010, 145:188-192.

1443. McIntyre RS, Alsuwaidan M, Goldstein BI, Taylor VH, Schaffer A, Beaulieu S, Kemp DE: The Canadian Network for Mood and Anxiety Treatments (CANMAT) task force recommendations for the management of patients with mood disorders and comorbid metabolic disorders. Ann Clin Psychiatry 2012, 24:69-81.

1444. Ramasubbu R, Taylor VH, Saaman Z, Sockalingham S, Li M, Patten S, Rodin G, Schaffer A, Beaulieu S, McIntyre RS: The Canadian Network for Mood and Anxiety Treatments (CANMAT) task force recommendations for the management of patients with mood disorders and select comorbid medical conditions. Ann Clin Psychiatry 2012, 24:91-109.

1445. Ramasubbu R, Beaulieu S, Taylor VH, Schaffer A, Mclntyre RS: The CANMAT task force recommendations for the management of patients with mood disorders and comorbid medical conditions: diagnostic assessment, and treatment principles. Ann Clin Psychiatry 2012, 24:82-90

1446. Engum A: The role of depression and anxiety in onset of diabetes in a large population-based study. J Psychosom Res 2007, 62:31-38

1447. Aujla N, Davies MJ, Skinner TC, Gray LJ, Webb DR, Srinivasan B, Khunti K: The association between anxiety and measures of glycaemia in a population-based diabetes screening programme. Diabet Med 2011, 28:785-788

1448. Serretti A, Mandelli L: Antidepressants and body weight: a comprehensive review and meta-analysis. J Clin Psychiatry 2010, 71:1259-1272

doi:10.1186/1471-244X-14-S1-S1

Cite this article as: Katzman et al:: Canadian clinical practice guidelines for the management of anxiety, posttraumatic stress and obsessivecompulsive disorders. BMC Psychiatry 2014 14(Suppl 1):S1. 